\title{
The Biogeochemistry of Lipid Derived Infochemical Signals in the Ocean
}

By

\author{
Bethanie Rachele Edwards
}

B.A., Hendrix College, 2009

Submitted in partial fulfillment of the requirements for the degree of

Doctor of Philosophy

at the

\section{MASSACHUSETTS INSTITUTE OF TECHNOLOGY}

and the

\section{WOODS HOLE OCEANOGRAPHIC INSTITUTION}

February 2016

(C) 2016 Bethanie Rachele Edwards

All rights reserved.

The author hereby grants to MIT and WHOI permission to reproduce and to distribute publicly paper and electronic copies of this thesis document in whole or in part in any medium now know or hereafter created.

Author

Joint Program in Oceanography/Applied Ocean Science and Engineering Massachusetts Institute of Technology and Woods Hole Oceanographic Institution January 26, 2016

Certified by

Dr. Benjamin A. S. Van Mooy Associate Scientist with Tenure of Marine Chemistry and Geochemistry, WHOI

Accepted by 


\title{
The Biogeochemistry of Lipid Derived Infochemical Signals in the Ocean
}

\author{
By
}

\author{
Bethanie Rachele Edwards \\ Submitted to the MIT/WHOI Joint Program in Oceanography in partial fulfillment of the \\ requirements for the degree of Doctor of Philosophy in the field of Chemical Oceanography \\ THESIS ABSTRACT
}

The role of oxylipins in ocean biogeochemistry was investigated using microcosm amendment experiments, environmental lipidomics, and culture based studies. Oxylipins are a bioactive class of secondary metabolites produced by diatoms and other eukaryotic phytoplankton. Previous research has focused mainly on one class of oxylipins, polyunsaturated aldehydes (PUAs), and their impacts on copepods. And few studies have looked at the impacts of oxylipins in situ. Here I show that oxylipins have the potential to impact carbon flux attenuation, oxylipin production in situ is linked to diatom bloom decline and viruses, and oxylipins deter microzooplankton grazing.

Sinking particles collected in the North Atlantic were determined to be hot spots for PUAs with concentrations in the micromolar range. Natural particle associated microbial communities exhibited a dose dependent response to PUAs. Stimulatory PUA concentrations ranged from 1-10 $\mu \mathrm{M}$, resulting in enhanced remineralization of organic matter by particle associated microbes. Thus, PUAs produced during bloom decline may lead to greater flux attenuation and nutrient recycling.

A novel lipidomics approach was applied along a cruise track in the California Coastal System revealing that canonical diatom free fatty acids and oxylipins dominated the dissolved lipidome and oxylipin abundance was correlated with diatom bloom demise as assessed by phaeophytin and biogenic Si. RNA viruses were likely the cause of diatom bloom demise and may have induced oxylipin production. The link between viruses and oxylipins represents a new infochemical signaling pathway in the ocean. Many oxylipins that are novel to the marine environment were also identified.

The dissolved lipidome was sampled during grazing experiments with the microzooplankton grazer Oxyrrhis marina and both wild type Phaeodactylum tricornutum and a chronically stressed, transgenic strain (PtNOA). Grazing was suppressed in the PtNOA treatments compared to the WT, likely due to upregulation of small unknown lipophilic molecules. This suggests that cellular stress and oxylipin production may deter microzooplankton grazing in the environment potentially altering the transfer of energy through the microbial food web.

By employing interdisciplinary approaches, we have learned that oxylipins production in situ is linked to bloom decline and the bioactivity of these compounds has significant implications for ocean biogeochemical cycles.

Thesis supervisor: Dr. Benjamin Van Mooy

Title: Associate Scientist with Tenure, Department of Marine Chemistry and Geochemistry, WHOI 


\section{Acknowledgments}

First and foremost, I would like to acknowledge my advisor, Ben Van Mooy for taking a chance on me. It took me a while to find the graduate program that was right for me. When I finally decided on WHOI, he welcomed me with open arms. Over the past five years, he has fostered my scientific creativity and independence. He hasn't always agreed with my ideas but he always allowed to me explore new techniques and take ownership of my research. Ben puts a great deal of effort into helping his students assimilate into the scientific community, urging us to attend conferences, introducing us to everyone that we meet, and including us in collaborations. I am most thankful for his generosity and thoughtful approach to advising. I was able to hone my writing skills and pen two first author papers while under his tutelage. It has been a pleasure working with you. Thank you for being an excellent advisor and friend.

I would also like to thank the rest of the Van Mooy lab who really are like family, a very welloiled, scientifically inclined family. Thank you, Helen Fredricks for answering my daily barrage of questions with a smile on your face and a drawer full of snacks. If I am ever half the lipid chemist you are, I'll die happy. Thank you, Justin Ossolinski for handling all the deck-ops with such great easy and organization. You were always quick to encourage me in the lab and at sea and that really made a difference. Thank you, Jamie for helping me get my head around lipidomics and R. You bring so much invaluable knowledge and know-how to the Van Mooy lab. I have no idea what my thesis would look like without your help. I'd also like to thank Kim Popendorf who was the senior graduate student when I started. You are a great mentor and friend. Your sunny disposition made the first couple of years of grad school much less daunting.

I could not have written this thesis without our collaborators through the Gordon and Betty Moore Foundation project: Kay Bidle, Matt Johnson, Tracy Mincer, and Assaf Vardi. I truly enjoyed going to sea with these people and their lab groups. Kay you have a passion for science and a refreshing attitude that I hope to channel as I move forward with my career. Thank you for all of your thoughtful comments on Chapter 2 and Chapter 4. Kim Thamatrakoln, you have been an invaluable collaborator and I look at you as a mentor. Thank you for all the career advice and good conversation at sea. And thank you for pushing me to incorporate viruses into Chapter 4 of this thesis; they stole the show! I look forward to working with you and Kay to get this DYEatom cruise data published in the near future. Thank you, Matt for setting up so many experiments for me to sample lipids from and for your patience. Also, thank you for chairing my committee and giving me such great feedback. You have piqued my interest in microzooplankton and I expect that that will follow me all the days of my scientific career.

I must thank the captains and crews of the R/V Knorr, R/V Point Sur, and R/V Atlantic Explorer on KN207-1, KN207-3, PS1312, and AEx1103. It was a joy sailing with you all and thank you for making our science possible, as well as, comfortable and safe. I would also like to thank my committee members: Liz Kujawinski, Mak Saito and Ed Delong. Their thoughtful critiques improved my thesis by leaps and bounds. 
The professors, mentors, and research advisors that I had throughout my undergraduate education were the ones that inspired me to start this path. They believed in me and equipped me with the critical thinking skills and a passion for science. Thank you Tom Goodwin, Linda GattiClark, Jen Dearolf, Walter Conely, Kara Lavender-Law, Robert Preston, Eric Webb, P. Dreux Chappell, and Jill Sohm. You were there at the beginning.

Last but certainly not least, I'd like to thank all of my friends and family for their support and encouragement on this long and arduous journey. Sadly, my grandmother, Judy Barker, did not make it to see this day. She encouraged me throughout my youth to forget about boys and focus on my career. I can't thank her enough for that and I hope that I have made her proud. I'd like to thank my local Woods Hole friends for making the last five years fun and entertaining. The ladies of Brazen Belles Burlesque made me forget all of my cares and just live (and dance) for the moment. You all are wonderfully spirited individuals and I am so lucky to have met you. Thank you to my friends in the Joint Program for commiserating with me, encouraging me, and inspiring me intellectually. And thank you, Charles Parker Walton for being my family and believing that I could do it even when you had no idea what it was that I was trying to do. You are my person.

This research was supported by a National Science Foundation Graduate Research Fellowship and grants from the U.S. National Science Foundation (OCE-1061883, OCE-1031143, and OCE 1155663), the Gordon and Betty Moore Foundation (GBMF3301), the U.S. Office of Naval Research (N000140910091), and the Simons Collaboration on Ocean Processes and Ecology (SCOPE). 


\section{Table of Contents}

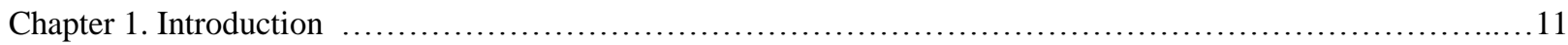

1. The bioactivity of oxylipins and implications for the biological pump ...........................13

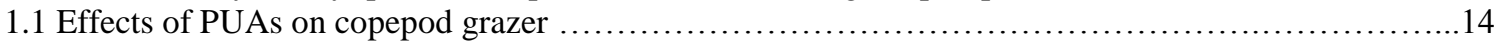

1.2 Effects of PUAs on eukaryotic phytoplankton........................................ 15

1.3 Effects of PUAs on bacteria........................................................ 16

1.4 Effects of PUAs on microzooplankton............................................... 17

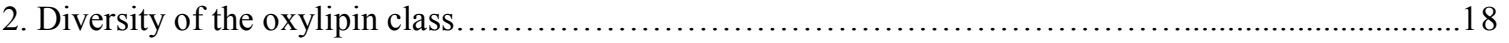

3. Oxylipin production tied to lipid remodeling and environmental stressors................................20

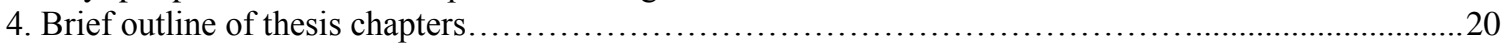

Chapter 2. Dose dependent regulation of microbial activity on sinking particles by polyunsaturated aldehydes:

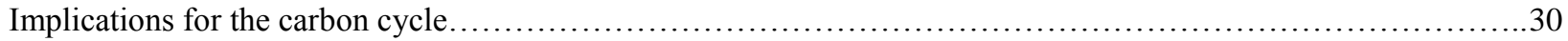

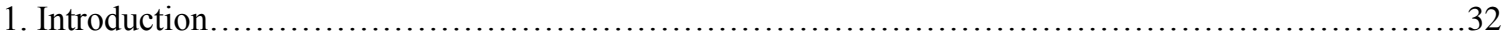

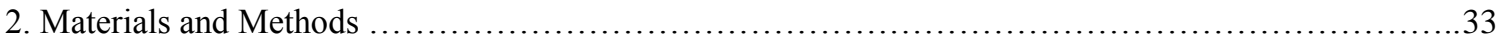

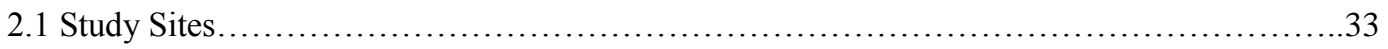

2.2 Experimental Design of Incubation Experiments ..................................... 33

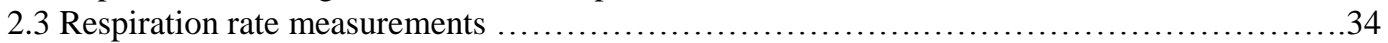

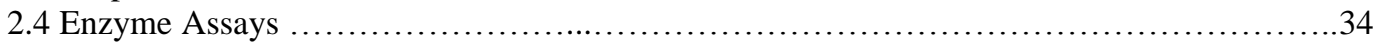

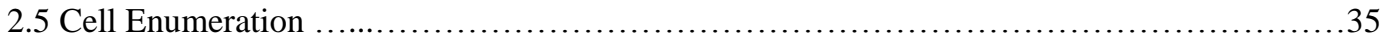

2.6 Estimation of the contribution of free-living vs. particle-associated bacteria in the incubation

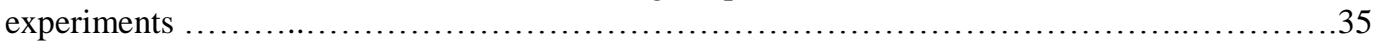

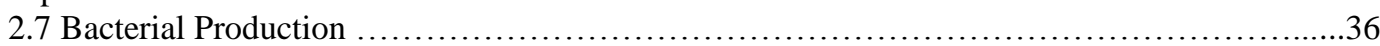

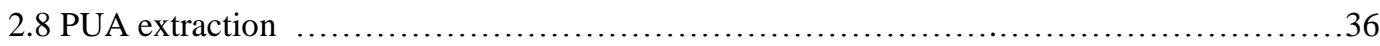

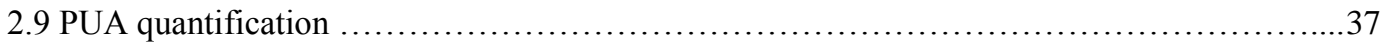

2.9.1 $\Delta\left[\mathrm{O}_{2}\right]$ observed compared to the $\mathrm{O}_{2}$ equivalent of $\Delta[\mathrm{PUA}] \ldots \ldots \ldots \ldots \ldots \ldots \ldots \ldots$

2.9.2 Estimation of PUA concentrations within sinking particles ...................... 38

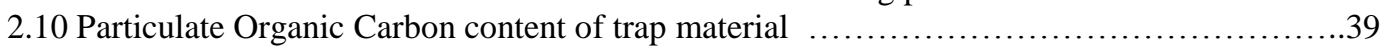

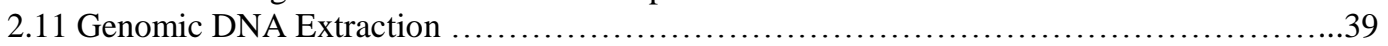

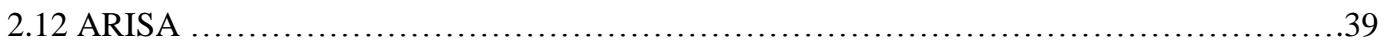

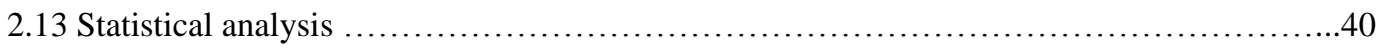

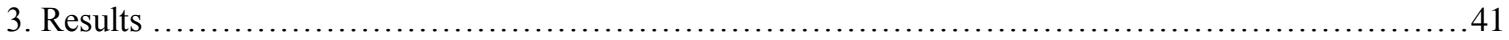

3.1 Exposure to PUAs affects changes in the rates of sinking POC remineralization .............41

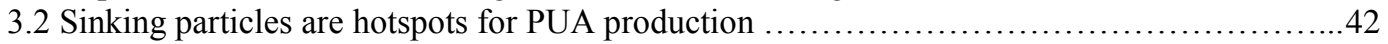

3.3 Shift in bacterial community upon exposure to PUAs . ..................................42

4. Discussion.

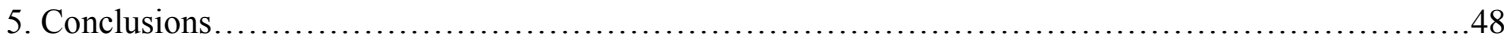

Chapter 3. Microzooplankton grazing and oxidative stress induce oxylipin production in Phaeodactylum tricornutum

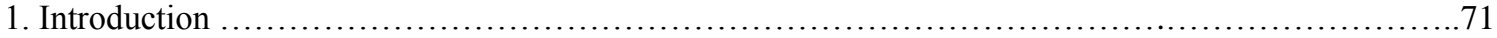

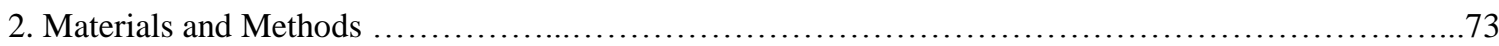

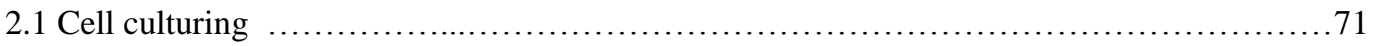

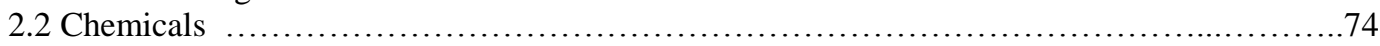

2.3 Laboratory grazing experiments, cell enumeration, and physiology $\ldots \ldots \ldots \ldots \ldots \ldots \ldots \ldots \ldots . \ldots \ldots$

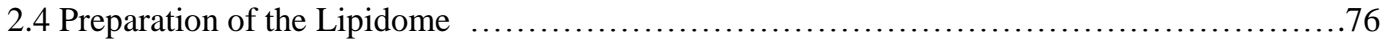

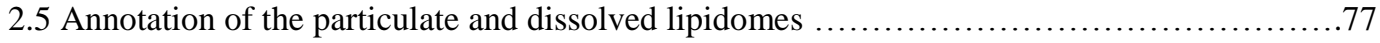

2.6 Normalization and relative quantification of molecular species ......................... 80

2.7 Structural and statistical analysis of the dissolved and particulate lipidomes ................80

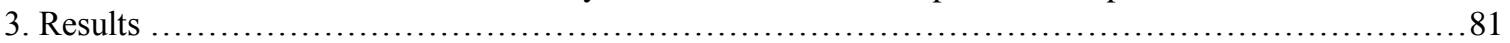

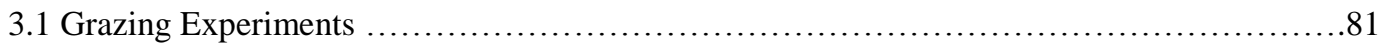

3.2 Dissolved Free Fatty Acids and Oxylipins ....................................... 82

3.2.1 3.2.1 Relative Quantification Using Comparative Lipidomics ....................82

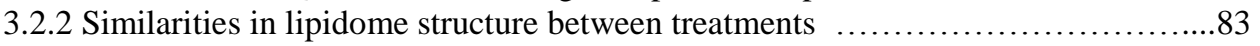

3.2.3 Oxylipin and free fatty acids significantly upregulated in response to stress .......83 
3.2.3.1 Differential production patterns of free fatty acids and oxylipin compounds in response to stress .......................................... 84

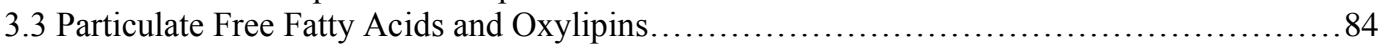

3.3.1 Untargeted annotation and relative quantification using comparative lipidomics...84

3.3.2 Similarities in lipidome structure between treatments $\ldots \ldots \ldots \ldots \ldots \ldots \ldots \ldots \ldots . \ldots 5$

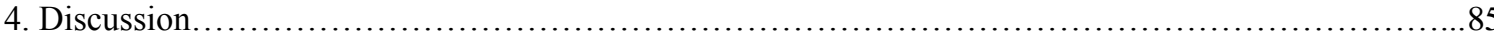

4.1 Linking phytoplankton stress to microzooplankton grazing deterrence.................... 85

4.2 Lipidomic differences between healthy and stressed cells................................ 86

4.2.1 Potential microzooplankton grazing deterrents in dissolved lipidome ...............87

4.2.2 Changes in particulate lipidome suggests grazing elicited production of $\mathrm{C} 20: 5$

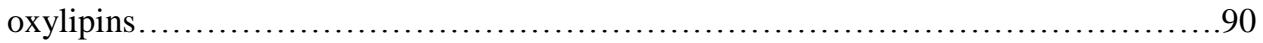

4.2.3 Additional insights from the particulate lipidome ............................ 90

4.3 Potentially bioactive molecules upregulated by grazing of WT cells......................99

4.3.1 C20:5 dihydroxy acids ...................................................... 93

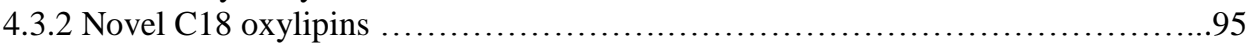

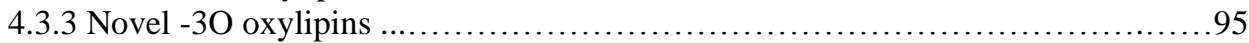

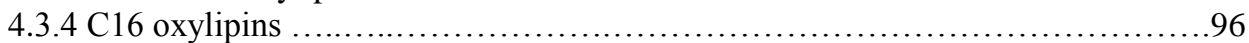

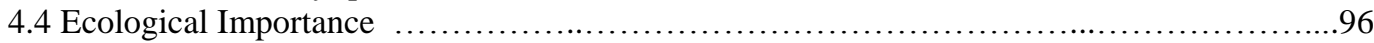

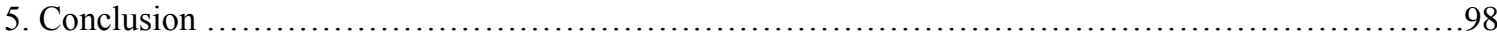

Chapter 4. Distribution and abundance of oxylipins in the California Coastal System corresponded with bloom

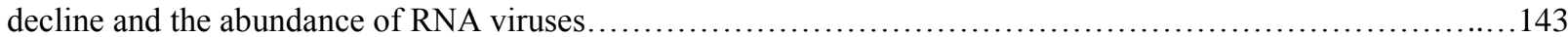

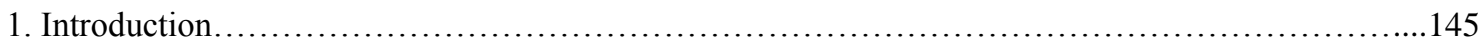

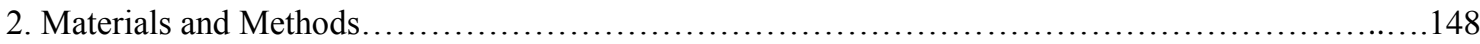

2.1 DYEatom cruise specifics and supplemental data collection ...........................148

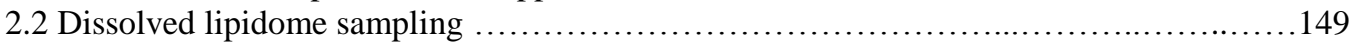

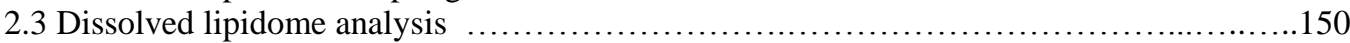

2.4 Dissolved lipidome annotation ....................................................

2.4.1 Functional group level annotation based on retention times of the standards....151 2.4.2 Functional level annotation based on theoretical retentions calculated from octan-

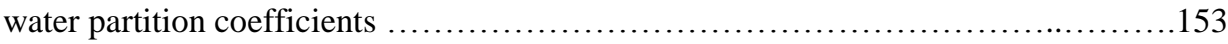

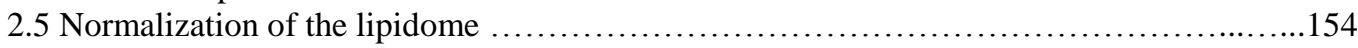

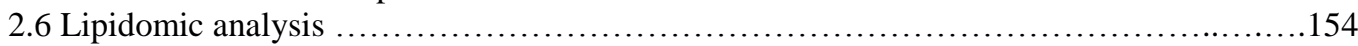

2.6.1 Richness and Diversity of the oxylipidome at each station .....................154

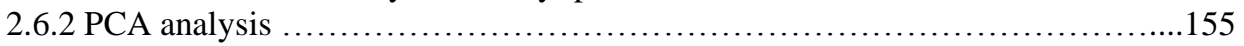

2.6.3 Similarity profile analysis ..............................................155

2.6.4 Permutational multivariate analysis of variance ..............................155

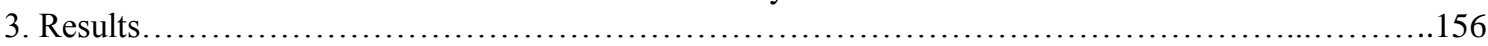

3.1 Cruise description and biogeochemical properties across the transect ................... 156

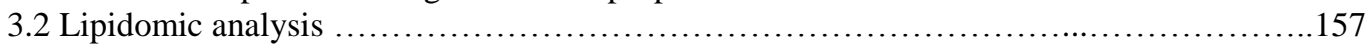

3.2.1 Most abundant compounds in the dissolved lipidome ........................157

3.2.2 Descriptive analyses of the dissolve lipidome .............................. 158

3.2.2.1 Significantly varying compounds in the dissolved lipidome ..................158

3.2.2.2 Principal component loadings of core supplemental variables underlying large

variation in the dissolved lipidome ................................................. 158

3.2.2.3 Correlations between non-core supplemental variables and the principal

components describing the dissolved lipidome revealed relationship with RNA viral

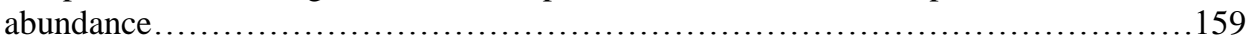

3.2.2.4 Level Scaling of the data and over all shifts in the dissolved lipidome...........160 
4. Discussion

4.1 Strong contribution from diatoms to the dissolved meta-lipidome

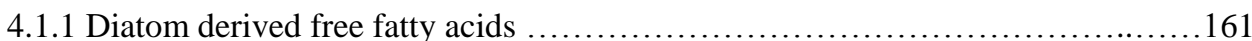

4.1.2 High abundance of non-volatile oxylipins derived from $\mathrm{C} 20: 5 \ldots \ldots \ldots \ldots \ldots \ldots . .162$

4.1.3 Minor C20:5 components of the dissolved lipidome ...........................164

4.2 Bioactivity of high abundance EPA derived non-volatile oxylipins .......................165

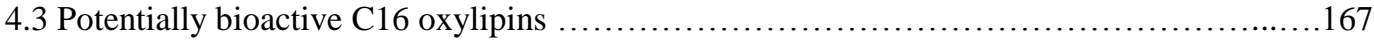

4.4 High abundance of a potentially novel algaecide in marine environments .................168 4.5 Shifts in the dissolved lipidome across the cruise transect driven by fluctuations in major free

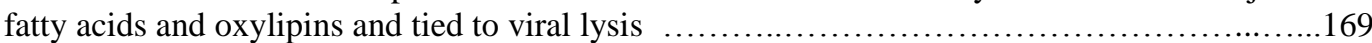

4.6 Grazing and Nutrient Stress .................................................. 172

4.7 Virally induced infochemical signaling: balancing mechanisms of diatom mortality ......173

4.7.1 Increased oxylipin production associated with RNA viruses decreases

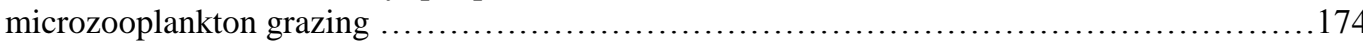

4.7.2 Increased oxylipin abundance associated with viruses may limit viral infection by removal of viruses from the upper ocean via aggregate formation and export ................175

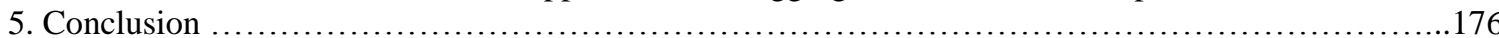

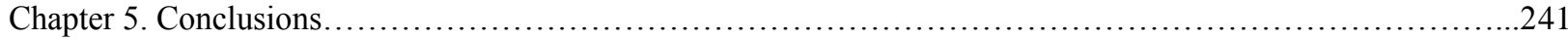

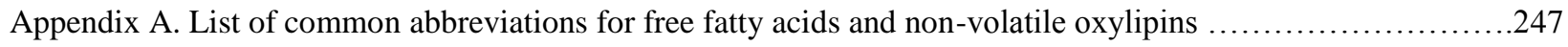

Appendix B. Comparison of solid phase extraction methods for sampling dissolved oxylipins and PUAs in seawater

Appendix C. Abiotic and exoenzymatic degradation of PUAs in natural seawater

Appendix D. The influence of PUAs on biogenic Si remineralization on sinking particles exported from Si-stressed phytoplankton communities

Appendix E. Preliminary analysis of oxylipins produced intracellularly during infection of Chaetoceros tenuissimus with RNA virus 


\section{Chapter 1}

Introduction to the biogeochemistry of lipid derived infochemicals in the ocean. 
1. The bioactivity of oxylipins and implications for the biological pump

Oxylipins are a family of biomolecules that are derived from lipids and often function as infochemicals between organisms. Infochemicals carry some information about the sender of the signal and cause an adaptive response in the receiver of the signal (ex: pheromones, kairomones, and allelochemicals). Oxylipin diversity and bioactivity have been well characterized in higher plants, animals, and macroalgae (Andreou et al., 2009). However, we are only beginning to understand the ecological impact of oxylipins produced by eukaryotic phytoplankton in situ. Many diatom species and some prymnesiophytes are known to produce oxylipins. These eukaryotic phytoplankton represent an important link in the global carbon cycle, fixing $\mathrm{CO}_{2}$ dissolved in the upper ocean into organic carbon, a portion of which will be exported to depth causing disequilibrium between the oceans and atmosphere and driving net absorption of $\mathrm{CO}_{2}$ by the ocean (Figure 1). However, this is a simplified view: the majority of organic carbon fixed by phytoplankton never makes it below 200m (Martin et al., 1987). This is due in large part to the intricate microbial loop and biological pump which rework particulate organic matter into dissolved organic matter, $\mathrm{CO}_{2}$, and microbial biomass (Azam et al., 1983; Fenchel, 2008). Even with such low export efficiency, 2 Gt of carbon fixed by phytoplankton is transferred from the atmosphere to the ocean annually (about 20\% of fossil fuel emissions) (Sabine et al., 2004).

Thus, understanding the factors that influence the fate of this carbon is imperative for parameterizing the global carbon cycle.

Metrics of biology and mineral ballasting within the water column such as biogenic $\mathrm{Si}$, particulate inorganic carbon, and chlorophyll do not adequately explain variations in export efficiency across the global ocean (Buesseler et al., 2007; Klaas and Archer, 2002; Le Moigne et al., 2014). Thus there has been a shift within the scientific community towards investigating the biological pump in terms of ecosystem function through community fingerprinting, functional genomics, transcriptomic, proteomics, and metabolomics. One arena of ecosystem function that has been largely overlooked is the influence of infochemical signals released by phytoplankton. Oxylipin infochemical signaling is a potential means of influencing ecosystem function because these compounds are produced by stressed, dying cells and impact multiple tiers of the oceanic trophic structure (Figure 2). 
Mechanisms of diatom mortality and organic carbon export include grazing, viral lysis, and nutrient stress. Both of which are associated with the production of polyunsaturated aldehydes (PUAs), a unique class of oxylipin in marine systems that has been studied extensively in culture (Pohnert, 2000; Ribalet et al., 2009; Vidoudez et al., 2011b; Wichard et al., 2007). To date more than fifty diatom species have been screened for PUA production, revealing that more than one-third of these species produce these potent compounds (Wichard et al., 2005).

PUAs were discovered in marine systems as scientists investigated the 'Paradox of the Plankton' which is based on the observation that copepods fed diatom diets exhibited lower reproductive success than those fed other eukaryotic phytoplankton (Ban and et al., 1997). At the time copepods were thought to be the main grazers of diatoms and it was confounding that copepods would choose a food source that inhibited their proliferation. Analysis of diatom extracts soon revealed that PUAs, namely two decatrienal isomers and one decadienal isomer, were the cause of decreased copepod recruitment in the Northern Adriatic (Miralto et al., 1999). And so began the age of PUA amendment experiments whereby PUAs were found to impact nearly every group of organisms in the marine microbial loop in a dose dependent fashion (Ianora and Miralto, 2010), cementing their importance in the marine carbon cycle.

\subsection{Effects of PUAs on copepod grazers}

PUAs decrease copepod reproductive success by causing teratogenic effects and inducing apoptosis in the offspring of parents fed PUA producing diatoms (Ianora and Miralto, 2010). Interestingly, PUAs do not consistently deter copepod grazing (Barreiro et al., 2011; Ianora et al., 2004; Juttner, 2005; Leising et al., 2005; Turner et al., 2001). In fact, copepod grazing on Procentrum minimum (a non-toxic dinoflagellate) was enhanced in the presence of PUAs and there have only been a few reports of PUAs causing immediate copepod mortality (Ka et al., 2014). Generally, the insidious effects of PUAs are latent and not considered a true chemical defense against copepods. However a handful of studies have found that micromolar level exposure to PUAs can decrease the survivorship of copepods, with males having a heightened sensitivity compared to females (Buttino et al., 2008; Carotenuto et al., 2011; Ka et al., 2014; Taylor et al., 2007). 
It was recently recognized that microzooplankton (e.g. dinoflagellates) are the dominate grazers of diatoms not mesozooplankton (e.g. copepods; Calbet, 2004), yet PUAs still impact copepod fecundity in situ. In addition to Miralto et al.'s (1999) initial report of PUA producing diatoms decreasing copepod hatching success in the Northern Adriatic, there have been corroborating field studies off the coast of Washington, in the upwelling zone off of Chile, and in the Baltic Sea (Ask et al., 2006; Halsband-Lenk et al., 2005; Vargas et al., 2006).

\subsection{Effects of PUAs on eukaryotic phytoplankton}

PUAs exuded by diatoms are not only detrimental to their predators but other phytoplankton as well. Ribalet et al. (2007a) conducted a study to determine the concentration dependent effect of three different PUAs on six species of phytoplankton. Addition of PUAs to cultures decreased growth rates and increased cell membrane permeability in all six species. However, the sensitivity of each species varied. Skeletonema marinoi was the most resistant strain tested and was significantly more tolerant of the PUAs that it was capable of producing (octadienal and heptadienal) compared to xenobiotic PUAs (decadienal). Species without structured or mineralized cell walls were the most sensitive to PUA addition, which is consistent with idea the PUAs cause harm to organisms by disrupting the cell membrane (Hutchinson et al., 1980). It was proposed that the differential sensitivity to PUAs may reflect the ability of phytoplankton to use PUAs as allelopathic compounds in order to compete with one another. In fact, Skeletonema marinoi responded to octadienal and heptadienal by increasing antioxidant activity and decreasing NO, whereas Phaeodactylum tricornutum (not known to make PUAs) perceived PUAs as allelochemicals, responding to decadienal by increasing NO production (Gallina et al., 2014).

Decadienal functions as a part of a nitric oxide (NO) mediated stress surveillance system in P. tricornutum (Vardi et al., 2008; Vardi et al., 2006). Exposure to low concentrations (0.66 $\mu \mathrm{M})$ of decadienal 'immunizes' the population to subsequent exposure to lethal doses $(13.2 \mu \mathrm{M})$, sending a NO signal throughout the population that can be observed through staining techniques (Vardi et al., 2006). However, un-primed exposure to lethal doses of decadienal induced an NO signaling cascade that led to cell death. In benthic diatoms, exposure to PUAs also induced NO 
signaling that resulted in biofilm formation and settling of cells (Leflaive and Ten-Hage, 2011).

The link to NO signaling is important since NO is a robust signaling molecule across all domains of life and likely underlies many of the bioactive effects of PUAs. Additionally, the dose dependence of PUA bioactivity within a diatom population suggests signaling functions of oxylipins outside of rudimental toxicity, as well as, a role for PUAs in phytoplankton community succession.

\subsection{Effects of PUAs on bacteria}

Marine bacteria in the water column can be subdivided into two broad groups: free-living and particle associated. Free-living bacteria tend to have a lower metabolic activity, utilizing dissolved organic matter in the ocean, whereas, particle associated bacteria tend to make a broad array of enzymes, working in consortia with other bacteria to degrade phytoplankton derived organic matter sinking through the oceans (Grossart et al., 2007). Marine bacteria are particularly important to the global carbon cycle, remineralizing upwards of $80 \%$ of organic matter in the ocean's interior (Helmke et al., 2010). Free-living and particle attached bacteria respond differently to PUA exposure. Free-living strains tend to exhibit decreased growth rates in response to PUAs (Ribalet et al., 2008). This effect is dose-dependent with higher PUA concentrations leading to a greater reduction in bacterial growth rate.

Prior to this thesis, particle associated bacteria had not be thoroughly investigated in the context of oxylipin infochemical signaling and organic matter remineralization. However, when cultures were challenged with PUA amendment, the growth of diatom associated strains were unaffected by PUAs and growth rates of Eudora adriatica and Alteromonas hispanica were actually enhanced (Ribalet et al., 2008). This suggests that bacterial species living in close proximity to PUA producers have substantially higher tolerances, potentially helping them carve out their ecological niche. In conjunction, Emiliana huxleyi and Phaeocystsi globosa also utilize infochemical signals to communicate with their bacterial epibionts, suggesting that phytoplankton-bacteria signaling may be common in the marine phycosphere (Seyedsayamdost et al., 2011; Sheik et al., 2014). 


\subsection{Effects of PUAs on microzooplankton}

The impact of oxylipins on microzooplankton is only beginning to be understood. Generally, the addition of PUAs decreased the growth rates of natural microzooplankton communities collected in the Chesapeake Bay and Atlantic coastal waters (Lavrentyev et al., 2015). However, the response of individual species was much more complex. There were eight microzooplankton species that responded negatively to PUAs with decreased growth rates at all of the concentrations tested. Four species exhibited enhanced growth rates at low concentrations $(2.2 \mathrm{nM})$ and decreased growth rates at medium $(5.5 \mathrm{nM})$ and high concentrations $(22 \mathrm{nM})$. Another ten species were characterized as having a weak response to PUA with decreased growth rates only at high PUA concentrations. Therefore, oxylipin production in situ would

likely impact the microzooplankton community in a species specific manner perhaps influencing community succession.

The impact of PUAs on microzooplankton grazing had not been specifically addressed previous to Chapter 3 of this thesis. But a recent study did find that compounds released by diatoms during large volume filtration inhibited microzooplankton grazing, eluding to oxylipin production by phytoplankton (Stoecker, 2015). Similarly, karlotoxins produced by some strains of the red tide algae, Karlodinium veneficum, were found to decrease Oxyrrhis marina grazing by $55 \%$ compared to grazing on non-toxic strains (Adolf et al., 2007). Conversely, DMSP produced by Emiliania huxleyi, another important phytoplankton in the marine environment, was recently found to enhance microzooplankton predation on virally infected cells (Breckels et al., 2011). Since compounds released by phytoplankton clearly impact microzooplankton grazers, the specifics of oxylipin infochemical signaling in mediating diatom microzooplankton interactions was a top priority going into this study.

Furthermore, the connections between PUAs and viruses have not been directly tested, representing one of the only aspects of the microbial trophic structure that has not been the focus of oxylipin research. However, a recent study in the northern Adriatic found that dissolved and particulate PUA concentrations were correlated with diatom cell lysis, suggesting that viral infection may contribute to PUA production (Ribalet et al., 2014). This is a likely hypothesis since oxylipin production is generally associated with membrane disruption. Chapter 4 explores infochemical signaling in the diatom rich California Coastal System and links between oxylipin 
concentrations in situ and the mechanisms of diatom mortality including viruses, nutrient stress, and grazing.

\section{Diversity of the oxylipin class}

The effects of PUAs described above likely represent just a fraction of bioactivity within the broad oxylipin class which also encompasses the structurally diverse non-volatile oxylipins (NVO). Figure 3 depicts a simplified view of the oxylipin biosynthetic pathway that will be outlined in more detail in later chapters. Oxylipin biosynthesis typically begins by liberating polyunsaturated fatty acids (PUFAs) from the membrane lipids by lipase activity. Next lipoxygenase enzymes (LOX) that are double bond specific add a hydroperoxy group. Oxylipin biosynthesis could stop at this point but often there are downstream modifications to the hydroperoxy group via allene oxide synthase (AOS), peroxidase, and hydroperoxy lyase (HPL) activity. AOS enzymes give rise to hydroxy epoxy acids, sometimes called epoxy alcohol acids. Peroxidases reduce hydroperoxy acids to hydroxy acids. HPL activity cleaves the hydroperoxy acid into two short chain oxylipins, a PUA and an oxo-acid. Additionally, hydroperoxy acids can undergo non-enzymatic isomerization to produce analogous keto-acids, sometimes referred to as an oxo-acid, although that nomenclature is reserve for keto groups occurring at a terminal carbon.

In diatoms, LOX enzymes can be specific for the $5^{\text {th }}, 6^{\text {th }}, 9^{\text {th }}, 11^{\text {th }}, 12^{\text {th }}, 14^{\text {th }}, 15^{\text {th }}, 16^{\text {th }}$, $17^{\text {th }}, 18^{\text {th }}$, or $20^{\text {th }}$ carbon of PUFAs (Andreou et al., 2009; Nanjappa et al., 2014). There are at least five fatty acids that serve as substrates in diatom oxylipin biosynthesis, eicosapentaenoic acid (20:5), arachidonic acid (20:4), hexadecatetraenoic acid (16:4), hexadecatrienoic acid (16:3), and docosahexadecaenoic acid (22:6). Given the range of enzyme specificity and substrates, the structural diversity of oxylipins grows quickly. Not to mention that these enzymes have catalytic plasticity and are often found as multifunctional/fusion proteins in nature, further increasing the diversity of enzymatic products (Andreou et al., 2009).

While non-volatile oxylipins do not have a long history in marine research, their bioactivity has garnered attention from those interested in mesozooplankton. Barreiro et al.(2011) found that NVO produced by Thalassiosira rotula, Skeletonema marinoi, and 
Skeletonema pseudocostatum decreased copepod hatching success in culture similarly to PUAs. Armed with this knowledge, Ianora et al (2015) took to the field, showing that NVOs decreased copepod hatching success in the northern Adriatic and were more abundant than PUAs over the entirety of the cruise. However, S. marinoi mesocosm experiments where NVOs were produced did not impact the reproduction of the copepod Calanus finmarchicus, implying a dose dependent response of copepods to NVOs and underscoring the important of absolute quantification (Gerecht et al., 2013).

Oxylipin profiling of diatom cultures has already revealed a large diversity of NVOs molecules (Barreiro et al., 2011; d'Ippolito et al., 2005; d'Ippolito et al., 2009; Fontana et al., 2007; Nanjappa et al., 2014; Pohnert, 2002) . However, the bioactivities of these compounds outside of copepod grazing are poorly understood and we know very little about their diversity in the marine environment with field studies only reporting summed NVO concentrations. Furthermore, previous studies that aimed to analyze PUAs and NVOs in the marine environment were fraught with sampling issues, the most serious being that oxylipins were typically sampled by concentrating cells and then subjecting them to multiple rounds of sonication or freeze-thaw cycles to elicit production. Therefore, many values reported in the literature represent the potential oxylipin concentration per cell if all of the cells were subject to sloppy grazing or lysis.

However, there has been a move towards measuring truly dissolved PUA concentrations in situ. Vidoudez et al (2011a) and Ribalet et al. (2014) sampled dissolved PUAs by pre-filtered seawater samples before using solid phase extraction methods paired with different inline derivatization steps. Samples were then quantified using GC-MS. At present, no studies have sought to analyze dissolved NVOs in the field. Using the methods presented in the marine oxylipin literature, at least three extraction and derivatization protocols would have to be employed to explore the diversity of compounds involved in the oxylipin biosynthetic pathway (Cutignano et al., 2011; Popendorf et al., 2013; Wichard et al., 2005). This presents a challenge at sea when time and space are in short supply, and hazardous extraction/derivatization methods are best avoided. Developing a method that could be used in the field to capture the breath of oxylipin diversity without pre-concentration or derivatization was an important aspect of Chapter 4 which described the diversity and distribution of oxylipins in the marine environment. 
3. Oxylipin production tied to lipid remodeling and environmental stressors

Lipids are biomolecules that serve structural and regulatory roles within cells. The structural role of lipids stems from their contribution to membrane bilayers. All cellular membranes are composed of a lipid bilayer with the hydrophilic head groups of intact polar lipids positioned towards the outside and hydrophobic free fatty acid tails positioned towards the inside of the bilayer. The specific lipids composing membrane bilayers vary greatly across taxa. However, within cells lipid remodeling can be used to deal with temperature stress, nutrient stress, and even viral stress (Converti et al., 2009; Dodson et al., 2014; Martin et al., 2011; Siron et al., 1989; Yu et al., 2009). Because lipids reflect the environmental conditions that they were synthesized in, they are often used in geochemistry as biomarkers for temperature, nutrient stress, and viral infection (Eglinton and Eglinton, 2008; Fulton et al., 2014; Van Mooy et al., 2006). While oxylipins production increases when the cell membrane is ruptured, the environmental factors presented above determine the composition of precursor molecules available for oxylipin biosynthesis and ultimately which suits of compounds are produced by wounded diatoms.

Nutrient stress exacts a strong control on the amount of PUAs released upon wounding of Skeletonema marinoi in culture and mesocosm experiments. Cells grown under Si and P limitation produced more PUAs that cells grown under nutrient replete conditions. The impact of $\mathrm{N}$-stress on PUA production varied between culture and mesocosm experiments, with $\mathrm{N}$-stressed cells in culture exhibiting decreased PUA production and $\mathrm{N}$-stressed cells in mesocosms exhibiting increased PUA production relative to the control (Ribalet et al., 2009; Vidoudez et al., 2011b). The variable response of $S$. marinoi to nutrient stress is not uncommon considering that identical strains of $S$. marinoi isolated during different times of the year produced different suites of oxylipins after sonication (Taylor, 2009). In conjunction, Nanjappa et al. (2014) found that Leptocylindrus sp. LOX enzymes varied seasonally, potentially explaining variations in PUA production amongst identical strains of diatoms. Additionally, UV stress and growth phase have been observed to impact potential PUA concentrations with more production upon UV irradiation and in stationary phase (Pacheco et al., 2014; Ribalet et al., 2007b). However, the controls on NVO production have not been explored and the controls on PUA production observed in culture and mesocosms have they been thoroughly investigated in situ where 
feedback loops may exist between the biogeochemical controls on oxylipins and the ecological implications of the bioactive molecules produced.

\section{Brief outline of thesis chapters}

The potential interconnections between oxylipins and biogeochemistry prompted a thorough investigation of infochemical signaling in the ocean, targeting understudied aspects of oxylipin bioactivity in the marine microbial loop (Figure 2). Chapter 2 was dedicated to understanding how natural particle associated bacteria respond to PUAs and how this impacts carbon export, as well as, determining PUA concentrations on sinking particles. PUA amendment experiments were conducted with sinking particles collected from six stations in the North Atlantic. Several parameters were measured to assess the response of particle associated bacteria to PUAs including: community respiration, enzymatic activity, bacterial cell abundance, bacterial production, and bacterial community structure. Additionally, the first measurements of PUAs on sinking particles were made. Chapter 3 sought to understand how the dissolved oxylipin pool produced by Phaeodactylum tricornutum changed with oxidative stress and microzooplankton grazing. A novel lipidomic method was employed to profile the suite of dissolved oxylipins produced. In conjunction, the impact of in vivo oxylipin production on microzooplankton grazing rates was assessed. The work undertaken in Chapter 4 endeavored to describe the diversity and distribution of oxylipins produced in the California Coastal System, a diatom dominated upwelling environment. The dissolved lipidomes collected were also linked to the biological processes in situ. Together the chapters of this thesis explore the importance of oxylipin infochemical signaling in the marine environment and elucidate previously unknown mechanisms by which oxylipins influence marine biogeochemistry.

\section{References}

Adolf, J.E., Krupatkina, D., Bachvaroff, T., Place, A.R., 2007. Karlotoxin mediates grazing by Oxyrrhis marina on strains of Karlodinium veneficum. Harmful Algae 6, 400-412.

Andreou, A., Brodhun, F., Feussner, I., 2009. Biosynthesis of oxylipins in non-mammals. Progress in Lipid Research 48, 148-170.

Ask, J., Reinikainen, M., Bamstedt, U., 2006. Variation in hatching success and egg production of Eurytemora affinis (Calanoida, Copepoda) from the Gulf of Bothnia, Baltic Sea, in relation to abundance and clonal differences of diatoms. Journal of Plankton Research 28, 683-694. 
Azam, F., Fenchel, T., Field, J.G., Gray, J.S., Meyerreil, L.A., Thingstad, F., 1983. The ecological role of watercolumn microbes in the sea. Marine Ecology Progress Series 10, 257-263.

Ban, S., et al., 1997. The paradox of diatom-copepod interactions. Marine Ecology Progress Series 157, 287-293.

Barreiro, A., Carotenuto, Y., Lamari, N., Esposito, F., D'Ippolito, G., Fontana, A., Romano, G., Ianora, A., Miralto, A., Guisande, C., 2011. Diatom induction of reproductive failure in copepods: The effect of PUAs versus non volatile oxylipins. Journal of Experimental Marine Biology and Ecology 401, 13-19.

Breckels, M.N., Roberts, E.C., Archer, S.D., Malin, G., Steinke, M., 2011. The role of dissolved infochemicals in mediating predator-prey interactions in the heterotrophic dinoflagellate Oxyrrhis marina. Journal of Plankton Research 33, 629-639.

Buesseler, K.O., Lamborg, C.H., Boyd, P.W., Lam, P.J., Trull, T.W., Bidigare, R.R., Bishop, J.K.B., Casciotti, K.L., Dehairs, F., Elskens, M., Honda, M., Karl, D.M., Siegel, D.A., Silver, M.W., Steinberg, D.K., Valdes, J., Van Mooy, B., Wilson, S., 2007. Revisiting carbon flux through the ocean's twilight zone. Science 316, 567-570.

Buttino, I., De Rosa, G., Carotenuto, Y., Mazzella, M., Ianora, A., Esposito, F., Vitiello, V., Quaglia, F., La Rotonda, M.I., Miralto, A., 2008. Aldehyde-encapsulating liposomes impair marine grazer survivorship. Journal of Experimental Biology 211, 1426-1433.

Calbet, A. and Landry, M.R., 2004. Phytoplankton growth, microzooplankton grazing, and carbon cycling in marine systems. Limnology and Oceanography 49, 51--57.

Carotenuto, Y., Ianora, A., Miralto, A., 2011. Maternal and neonate diatom diets impair development and sex differentiation in the copepod Temora stylifera. Journal of Experimental Marine Biology and Ecology 396, 99-107.

Converti, A., Casazza, A.A., Ortiz, E.Y., Perego, P., Del Borghi, M., 2009. Effect of temperature and nitrogen concentration on the growth and lipid content of Nannochloropsis oculata and Chlorella vulgaris for biodiesel production. Chemical Engineering and Processing 48, 1146-1151.

Cutignano, A., Lamari, N., d'Ippolito, G., Manzo, E., Cimino, G., Fontana, A., 2011. Lipoxygenase products in marine diatomsL A concise analytical method to exlore the functional potenial of oxylipins. Journal of Phycology 47, 233-243.

d'Ippolito, G., Cutignano, A., Briante, R., Febbraio, F., Cimino, G., Fontana, A., 2005. New C16 fatty-acid-based oxylipin pathway in the marine diatom Thalassiosira rotula. Org Biomol Chem 3, 4065-4070.

d'Ippolito, G., Lamari, N., Montresor, M., Romano, G., Cutignano, A., Gerecht, A., Cimino, G., Fontana, A., 2009. 15S-Lipoxygenase metabolism in the marine diatom Pseudo-nitzschia delicatissima. New Phytologist 183, 10641071.

Dodson, V.J., Mouget, J.-L., Dahmen, J.L., Leblond, J.D., 2014. The long and short of it: temperature-dependent modifications of fatty acid chain length and unsaturation in the galactolipid profiles of the diatoms Haslea ostrearia and Phaeodactylum tricornutum. Hydrobiologia 727, 95-107.

Eglinton, T.I., Eglinton, G., 2008. Molecular proxies for paleoclimatology. Earth and Planetary Science Letters 275, $1-16$.

Fenchel, T., 2008. The microbial loop-25 years later. Journal of Experimental Marine Biology and Ecology 366, 99 103.

Fontana, A., d'Ippolito, G., Cutignano, A., Romano, G., Lamari, N., Gallucci, A.M., Cimino, G., Miralto, A., Ianora, A., 2007. LOX-induced lipid peroxidation mechanism responsible for the detrimental effect of marine diatoms on Zooplankton grazers. Chembiochem 8, 1810-1818. 
Fulton, J.M., Fredricks, H.F., Bidle, K.D., Vardi, A., Kendrick, B.J., DiTullio, G.R., Van Mooy, B.A.S., 2014. Novel molecular determinants of viral susceptibility and resistance in the lipidome of Emiliania huxleyi. Environmental Microbiology 16, 1137-1149.

Gallina, A.A., Brunet, C., Palumbo, A., Casotti, R., 2014. The Effect of Polyunsaturated Aldehydes on Skeletonema marinoi (Bacillariophyceae): The Involvement of Reactive Oxygen Species and Nitric Oxide. Marine Drugs 12, 4165-4187.

Gerecht, A., Carotenuto, Y., Ianora, A., Romano, G., Fontana, A., d'Ippolito, G., Jakobsen, H.H., Nejstgaard, J.C., 2013. Oxylipin production during a mesocosm bloom of Skeletonema marinoi. Journal of Experimental Marine Biology and Ecology 446, 159-165.

Grossart, H.-P., Tang, K.W., Kiorboe, T., Ploug, H., 2007. Comparison of cell-specific activity between free-living and attached bacteria using isolates and natural assemblages. Fems Microbiology Letters 266, 194-200.

Halsband-Lenk, C., Pierson, J.J., Leising, A.W., 2005. Reproduction of Pseudocalanus newmani (Copepoda : Calanoida) is deleteriously affected by diatom blooms - A field study. Progress in Oceanography 67, 332-348.

Helmke, P., Neuer, S., Lomas, M.W., Conte, M., Freudenthal, T., 2010. Cross-basin differences in particulate organic carbon export and flux attenuation in the subtropical North Atlantic gyre. Deep-Sea Research Part IOceanographic Research Papers 57, 213-227.

Hutchinson, T.C., Hellebust, J.A., Tam, D., Mackay, D., Mascarenhas, R.A., Shiu, W.Y., 1980. The correlation of the toxicity to algae of hydrocarbons and halogenated hydrocarbons with their physical-chemical properties, Hydrocarbons and halogenated hydrocarbons in the aquatic environment. Springer, pp. 577-586.

Ianora, A., Bastianini, M., Carotenuto, Y., Casotti, R., Roncalli, V., Miralto, A., Romano, G., Gerecht, A., Fontana, A., Turner, J.T., 2015. Non-volatile oxylipins can render some diatom blooms more toxic for copepod reproduction. Harmful Algae 44, 1-7.

Ianora, A., Miralto, A., 2010. Toxigenic effects of diatoms on grazers, phytoplankton and other microbes: a review. Ecotoxicology 19, 493-511.

Ianora, A., Miralto, A., Poulet, S.A., Carotenuto, Y., Buttino, I., Romano, G., Casotti, R., Pohnert, G., Wichard, T., Colucci-D'Amato, L., Terrazzano, G., Smetacek, V., 2004. Aldehyde suppression of copepod recruitment in blooms of a ubiquitous planktonic diatom. Nature 429, 403-407.

Juttner, F., 2005. Evidence that polyunsaturated aldehydes of diatoms are repellents for pelagic crustacean grazers. Aquatic Ecology 39, 271-282.

Ka, S., Carotenuto, Y., Romano, G., Hwang, J.-S., Buttino, I., Ianora, A., 2014. Impact of the diatom-derived polyunsaturated aldehyde 2-trans,4-trans decadienal on the feeding, survivorship and reproductive success of the calanoid copepod Temora stylifera. Marine Environmental Research 93, 31-37.

Klaas, C., Archer, D.E., 2002. Association of sinking organic matter with various types of mineral ballast in the deep sea: Implications for the rain ratio. Global Biogeochemical Cycles 16, 63-61-63-14.

Lavrentyev, P.J., Franze, G., Pierson, J.J., Stoecker, D.K., 2015. The Effect of Dissolved Polyunsaturated Aldehydes on Microzooplankton Growth Rates in the Chesapeake Bay and Atlantic Coastal Waters. Marine Drugs 13, 28342856.

Le Moigne, F.A.C., Pabortsava, K., Marcinko, C.L.J., Martin, P., Sanders, R.J., 2014. Where ismineral ballast important for surface export of particulate organic carbon in the ocean? Geophysical Research Letters 41, 84608468. 
Leflaive, J., Ten-Hage, L., 2011. Effects of 2E,4E-decadienal on motility and aggregation of diatoms and on biofilm formation. Microb Ecol 61, 363-373.

Leising, A.W., Pierson, J.J., Halsband-Lenk, C., Horner, R., Postel, J., 2005. Copepod grazing during spring blooms: Does Calanus pacificus avoid harmful diatoms? Progress in Oceanography 67, 384-405.

Martin, J.H., Knauer, G.A., Karl, D.M., Broenkow, W.W., 1987. VERTEX - Carbon cycling in the Northeast Pacific. Deep-Sea Research Part a-Oceanographic Research Papers 34, 267-285.

Martin, P., Van Mooy, B.A.S., Heithoff, A., Dyhrman, S.T., 2011. Phosphorus supply drives rapid turnover of membrane phospholipids in the diatom Thalassiosira pseudonana. ISME J 5, 1057-1060.

Miralto, A., Barone, G., Romano, G., Poulet, S.A., Ianora, A., Russo, G.L., Buttino, I., Mazzarella, G., Laabir, M., Cabrini, M., 1999. The insidious effect of diatoms on copepod reproduction. Nature 402, 173-176.

Nanjappa, D., \#039, Ippolito, G., Gallo, C., Zingone, A., Fontana, A., 2014. Oxylipin Diversity in the Diatom Family Leptocylindraceae Reveals DHA Derivatives in Marine Diatoms. Marine Drugs 12, 368.

Pacheco, L., Uribe, E., Pino, J., Troncoso, J., Quiróz, A., 2014. The Effect of UV Light and $\mathrm{CO}_{2}$ in the Production of Polyunsaturated Aldehydes in Skeletonema costatum (Bacillariophycea). American Journal of Plant Science 5, 3632-3641.

Pohnert, G., 2000. Wound-Activated Chemical Defense in Unicellular Planktonic Algae. Angewandte Chemie International Edition 39, 4352--4354.

Pohnert, G., 2002. Phospholipase A2 activity triggers the wound-activated chemical defense in the diatom Thalassiosira rotula. Plant Physiol 129, 103-111.

Popendorf, K.J., Fredricks, H.F., Van Mooy, B.A., 2013. Molecular ion-independent quantification of polar glycerolipid classes in marine plankton using triple quadrupole MS. Lipids 48, 185-195.

Ribalet, F., Bastianini, M., Vidoudez, C., Acri, F., Berges, J., Ianora, A., Miralto, A., Pohnert, G., Romano, G., Wichard, T., Casotti, R., 2014. Phytoplankton Cell Lysis Associated with Polyunsaturated Aldehyde Release in the Northern Adriatic Sea. PLoS ONE 9, e85947.

Ribalet, F., Berges, J.A., Ianora, A., Casotti, R., 2007a. Growth inhibition of cultured marine phytoplankton by toxic algal-derived polyunsaturated aldehydes. Aquatic Toxicology 85, 219-227.

Ribalet, F., Intertaglia, L., Lebaron, P., Casotti, R., 2008. Differential effect of three polyunsaturated aldehydes on marine bacterial isolates. Aquatic Toxicology 86, 249-255.

Ribalet, F., Vidoudez, C., Cassin, D., Pohnert, G., Ianora, A., Miralto, A., Casotti, R., 2009. High plasticity in the production of diatom-derived polyunsaturated aldehydes under nutrient limitation: physiological and ecological implications. Protist 160, 444-451.

Ribalet, F., Wichard, T., Pohnert, G., Ianora, A., Miralto, A., Casotti, R., 2007b. Age and nutrient limitation enhance polyunsaturated aldehyde production in marine diatoms. Phytochemistry 68, 2059-2067.

Sabine, C.L., Feely, R.A., Gruber, N., Key, R.M., Lee, K., Bullister, J.L., Wanninkhof, R., Wong, C.S., Wallace, D.W.R., Tilbrook, B., Millero, F.J., Peng, T.H., Kozyr, A., Ono, T., Rios, A.F., 2004. The oceanic sink for anthropogenic CO2. Science 305, 367-371.

Seyedsayamdost, M.R., Carr, G., Kolter, R., Clardy, J., 2011. Roseobacticides: Small Molecule Modulators of an Algal-Bacterial Symbiosis. Journal of the American Chemical Society 133, 18343-18349. 
Sheik, A.R., Brussaard, C.P.D., Lavik, G., Lam, P., Musat, N., Krupke, A., Littmann, S., Strous, M., Kuypers, M.M.M., 2014. Responses of the coastal bacterial community to viral infection of the algae Phaeocystis globosa. Isme Journal 8, 212-225.

Siron, R., Giusti, G., Berland, B., 1989. Changes in the fatty-acid composition of Phaeodactylum tricornutum and Dunaliella tertiolecta during growth and under phosphorous deficiency. Marine Ecology Progress Series 55, 95-100.

Stoecker, D.K., Nejstgaard, J.C., Mashusoodhanan, R., Pohnert, G., Wolfram, S., Jakobsen, H.H., Šulčius, S., and Larsen, A., 2015. Underestimation of microzooplankton grazing in dilution experiments due to inhibition of phytoplankton growth. Limnology and Oceanography 60, 1426--1438.

Taylor, R.L., Caldwell, G.S., Dunstan, H.J., Bentley, M.G., 2007. Short-term impacts of polyunsaturated aldehydeproducing diatoms on the harpacticoid copepod, Tisbe holothuriae. Journal of Experimental Marine Biology and Ecology 341, 60-69.

Taylor, R.L., AbrahamssonK., Godhe, A., Wa“ngberg, S., 2009. Seasonal Variability in Polyunsaturated Aldehyde Production Potential among Strains of Skeletonema Marinoi (Bacillariophyceae)1. Journal of Phycology 45, 46--53.

Turner, J.T., Ianora, A., Miralto, A., Laabir, M., Esposito, F., 2001. Decoupling of copepod grazing rates, fecundity and egg-hatching success on mixed and alternating diatom and dinoflagellate diets. Marine Ecology Progress Series 220, 187-199.

Van Mooy, B.A.S., Rocap, G., Fredricks, H.F., Evans, C.T., Devol, A.H., 2006. Sulfolipids dramatically decrease phosphorus demand by picocyanobacteria in oligotrophic marine environments. Proceedings of the National Academy of Sciences of the United States of America 103, 8607-8612.

Vardi, A., Bidle, K.D., Kwityn, C., Hirsh, D.J., Thompson, S.M., Callow, J.A., Falkowski, P., Bowler, C., 2008. A diatom gene regulating nitric-oxide signaling and susceptibility to diatom-derived aldehydes. Curr Biol 18, 895-899.

Vardi, A., Formiggini, F., Casotti, R., De Martino, A., Ribalet, F., Miralto, A., Bowler, C., 2006. A Stress

Surveillance System Based on Calcium and Nitric Oxide in Marine Diatoms. PLoS Biol 4, e60.

Vargas, C.A., Escribano, R., Poulet, S., 2006. Phytoplankton food quality determines time windows for successful zooplankton reproductive pulses. Ecology 87, 2992-2999.

Vidoudez, C., Casotti, R., Bastianini, M., Pohnert, G., 2011a. Quantification of dissolved and particulate polyunsaturated aldehydes in the Adriatic Sea. Marine drugs 9, 500-513.

Vidoudez, C., Nejstgaard, J.C., Jakobsen, H.H., Pohnert, G., 2011b. Dynamics of Dissolved and Particulate Polyunsaturated Aldehydes in Mesocosms Inoculated with Different Densities of the Diatom Skeletonema marinoi. Marine Drugs 9, 345-358.

Wichard, T., Gerecht, A., Boersma, M., Poulet, S.A., Wiltshire, K., Pohnert, G., 2007. Lipid and fatty acid composition of diatoms revisited: rapid wound-activated change of food quality parameters influences herbivorous copepod reproductive success. ChemBioChem 8, 1146-1153.

Wichard, T., Poulet, S.A., Pohnert, G., 2005. Determination and quantification of $\alpha, \beta, \gamma, \delta$-unsaturated aldehydes as pentafluorobenzyl-oxime derivates in diatom cultures and natural phytoplankton populations: application in marine field studies. Journal of Chromatography B 814, 155-161.

Yu, E.T., Zendejas, F.J., Lane, P.D., Gaucher, S., Simmons, B.A., Lane, T.W., 2009. Triacylglycerol accumulation and profiling in the model diatoms Thalassiosira pseudonana and Phaeodactylum tricornutum (Baccilariophyceae) during starvation. Journal of Applied Phycology 21, 669-681. 


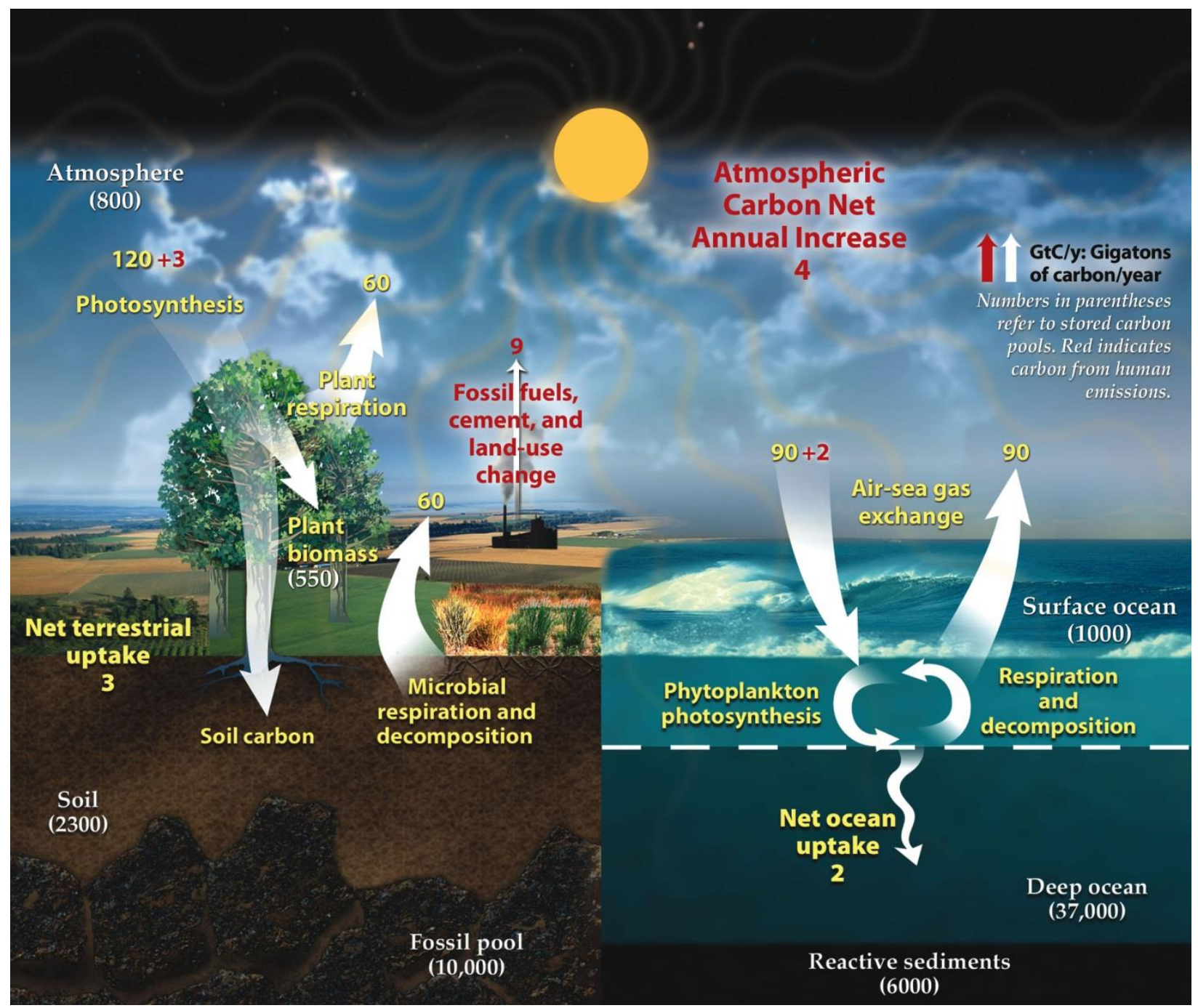

Figure 1. Global carbon cycle depicting the flow of carbon through terrestrial, atmospheric, and

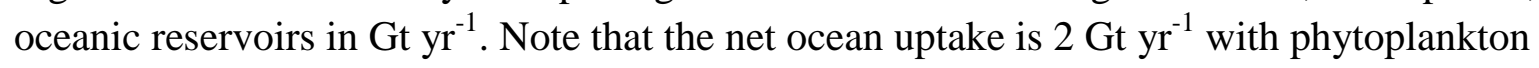
photosynthesis barely outpacing respiration and decomposition. (credit: Office of Biological Research of the U.S. Department of Energy Office of Science) 


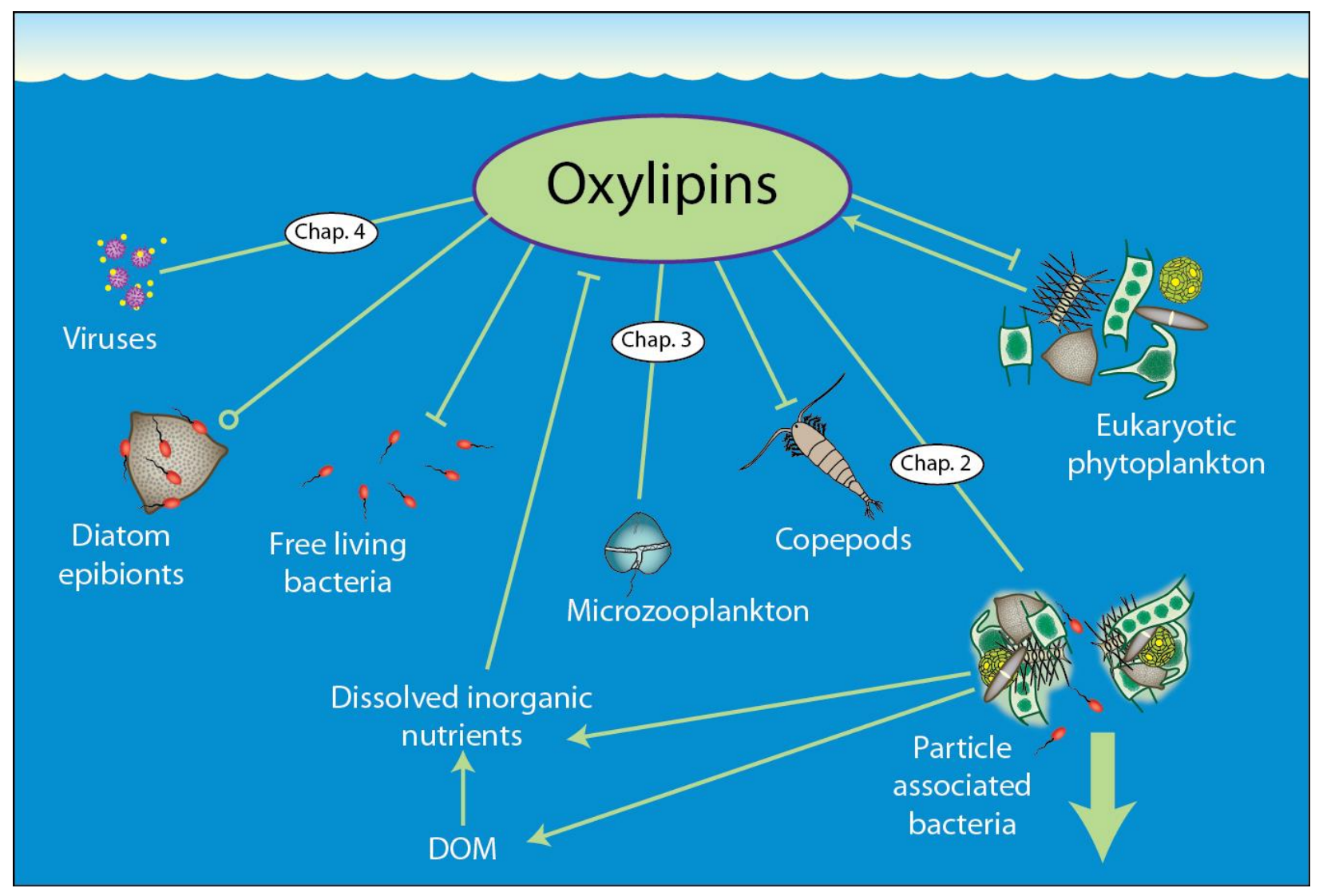

Figure 2. The impact of oxylipins on various levels of the microbial food web. The impact of oxylipins on viruses had not been previously investigated. Diatom associated bacterial isolates exhibit a high tolerance to PUAs although some bacteria strains exhibit enhanced growth (Ribalet et al., 2008). The growth of free living bacterial strains is generally inhibited by PUAs. Microzooplankton exhibit decreased growth rate when exposed to PUAs but the impact of grazing has not determined (Lavrentyev et al., 2015). When exposed to PUAs and NVOs copepods exhibit decreased reproductive success (Miralto et al., 1999, Barreiro et al., 2011, Ianora et al., 2015). The impact of oxylipins on sinking particles and the associated microbes had not been previously investigated. Phytoplankton produce oxylipins and exposure to PUAs generally causes growth inhibition but at sub-lethal doses PUAs can be used as a stress surveillance signal (Ribalet et al., 2007a; Vardi et al., 2006) 

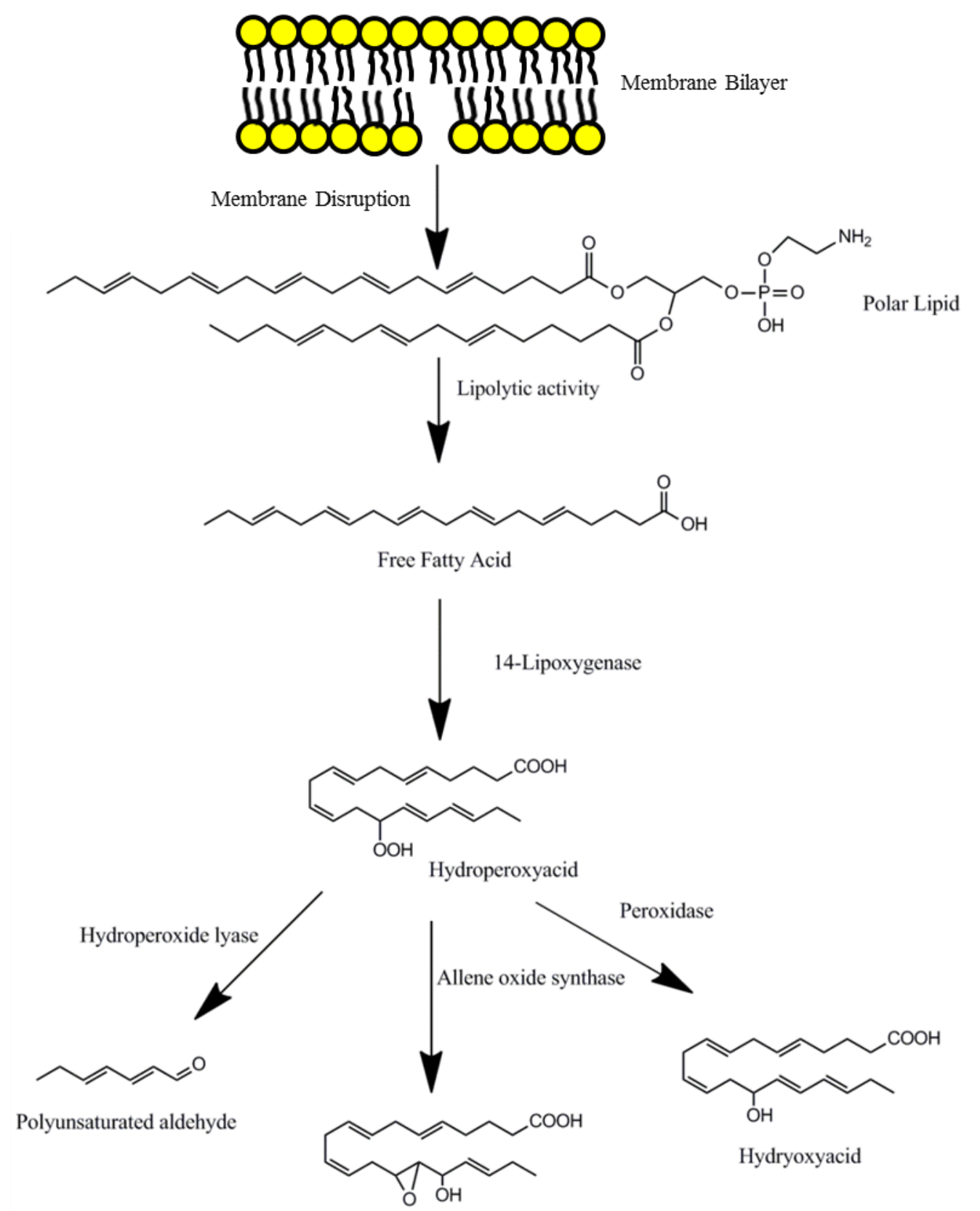

Epoxy alcohol

Figure 3. Simplified oxylipin biosynthetic pathway in diatoms displaying the diversity of potential compounds. Oxylipins production via the $\mathrm{C} 20: 5$ pathway is displayed here but $\mathrm{C} 22: 6$, C20:4, C16:3, and C16:4 fatty acids can also serve as substrates for oxylipin enzymes. Furthermore, the lipoxygenase enzymes can add hydroperoxy groups to a variety of carbons, not just the $14^{\text {th }}$ as displayed here. 


\section{Chapter 2}

Dose dependent regulation of microbial activity on sinking particles by polyunsaturated aldehydes: Implications for the carbon cycle

Published as: Edwards, B.R., Bidle, K.D., Van Mooy, B.A.S., 2015. Dose-dependent regulation of microbial activity on sinking particles by polyunsaturated aldehydes: Implications for the carbon cycle. Proceedings of the National Academy of Sciences 112, 5909-5914. 


\section{Introduction}

Planktonic microbes in the world's oceans play a major role in the global carbon cycle. Through photosynthesis, phytoplankton convert carbon dioxide into particulate organic carbon (POC), which then has the potential to sink to the deep-sea. This process is opposed by zooplankton and heterotrophic bacteria, which, as agents of respiration, degrade organic matter and convert it back into carbon dioxide. In addition to respiring POC, heterotrophic bacteria that are associated with sinking POC utilize membrane-bound ectohydrolytic enzymes that affect the disaggregation of POC into smaller non-sinking particles and dissolved organic carbon (DOC) (Smith et al., 1995). Despite the long-recognized role of particle-associated bacteria (Azam et al., 1994), the relationships between the activities of these bacteria, particle properties, and the time and depth scales of sinking POC degradation, disaggregation, and respiration remain poorly constrained. Although the molecular-level composition of sinking POC has been used to identify its phytoplanktonic sources and to constrain the timescales of its degradation by heterotrophic bacteria (Goutx and al, 2007; Van Mooy et al., 2002; Wakeham et al., 1997) none of these studies have accounted for the potential impacts of bioactive molecules within sinking POC on the activities of particle-associated bacteria.

Diatoms are key members of the phytoplanktonic communities across the world's ocean and are known to produce a large diversity of organic molecules, including many that are bioactive. Polyunsaturated aldehydes (PUAs) have received particular interest. The stage for discovery of these molecules was set in the 1990s, when a group of researchers advanced the "paradox of diatom-copepod interactions" - the observation that copepods, which prey on diatoms, exhibited decreased reproductive success when exclusively fed diatoms (Ban and et al., 1997; Ianora and Poulet, 1993; Poulet et al., 1995). Miralto et al. later purified PUAs from diatom cultures and observed arrested embryogenesis of copepod eggs that were exposed to these compounds (Miralto et al., 1999). PUA production is now a well-characterized, stress surveillance response to wounding during grazing and to nutrient depletion, both of which are bloom termination mechanisms (Schilmiller and Howe, 2005; Wichard et al., 2007).

It has been proposed that PUAs also mediate phytoplankton bloom dynamics by impacting other members of the marine planktonic community, aside from zooplankton. In culture conditions, many eukaryotic phytoplankton experience a decrease in growth rate when 
exposed to PUAs (Casotti et al., 2005; Ribalet et al., 2007a). Isolated bacterial strains demonstrate a varied response to PUAs, whereby diatom-associated isolates are generally unaffected while other strains exhibit either dose dependent decreases or increases in growth rate in response to PUAs (Ribalet et al., 2008). Although many culture studies have been conducted on zooplankton, phytoplankton, and bacterial isolates - all important players in the microbial loop - there have been few attempts to study the impact of PUAs on these trophic levels in natural marine ecosystems under in situ condition (Balestra et al., 2011; Ianora and Miralto, 2010). Surveys of water column concentrations of PUAs suggest that concentrations are generally much lower than levels required to elicit responses in phytoplankton, zooplankton, or bacteria (Bartual and al, 2014; Vidoudez et al., 2011). Consequently, the impact of PUAs on the marine carbon cycle remains an open question.

\section{Materials and Methods}

\subsection{Study Sites}

Incubation experiments exposing sinking particles to varying concentrations of PUAs were conducted on three different cruises in the Atlantic. Sinking particles were collected by a surface tethered net-trap deployed at $150 \mathrm{~m}$ (Peterson et al., 2005). Deployments were 24 hours long. A sonar triggered mechanism closed the traps before retrieval, such that only particulate matter sinking to $150 \mathrm{~m}$ was collected. These cruises transected three different regions: the Sargasso Sea (SS), the temperate west North Atlantic (TWNA), and the Subarctic North Atlantic (SANA) (Fig. 1). Due to the intensive sampling of the incubation experiments, the set of parameters measured varied between each experiment. Please see Table 1 for a list of measurements made during each experiment.

\subsection{Experimental Design of Incubation Experiments}

First, seawater collected from $150 \mathrm{~m}$ during a CTD cast directly before trap retrieval was filtered through a $0.22 \mu \mathrm{m}$ bottle top filter (Corning) and stored at in situ temperature until the incubations were started. Trap material was evenly distributed amongst four $500 \mathrm{~mL}$ Nalgene bottles, which were designated for each of the four treatments. Filtered seawater was added to each bottle to a final volume of $500 \mathrm{~mL}$. In incubations SS2, SS3, TWNA 1, TWNA 2, and SANA 1 trap material was diluted by 2 fold with filtered seawater whereas, dilution factor was 
15-fold in incubation SS1. Then the appropriate volume of PUA stock solution (equal parts heptadienal, octadienal, and decadienal) was added to each bottle to reach the desired concentrations of $0,1,10$, and $100 \mu \mathrm{M}$ PUA. After gently shaking each treatment, $150 \mathrm{~mL}$ of each treatment was transferred into three gas-tight biological oxygen demand bottles (i.e. triplicate incubations for each treatment). The BOD bottles were incubated in the dark for 24 hours at in situ temperature.

\subsection{Respiration rate measurements}

Respiration rates were determined by measuring the decrease in $\mathrm{O}_{2}$ concentration within each triplicate over the 24 hour incubation period. The BOD bottles were equipped with oxygen optode minisensors (PreSens) which allowed us to measure the $\mathrm{O}_{2}$ concentrations semicontinuously throughout the course of the incubation period. Measurements were taken at $\mathrm{t}=\mathrm{initial}, \mathrm{t}=1$ hour, $\mathrm{t}=4$ hours, and approximately every 6 hours thereafter. For further detail on this method please refer to Edwards et al. (2011).

\subsection{Enzyme Assays}

At the end of the 24 hour incubation period, $10 \mathrm{~mL}$ of each incubation was sampled for ectohydrolytic enzymatic activity by measuring the cleavage kinetics of model fluorgenic substrates. Respective enzymatic activity (mol substrate $\mathrm{L}^{-1} \mathrm{hr}^{-1}$ ) of alkaline phosphatase (APase), lipase, alpha-glucosidase, and peptidase were determined by measuring the hydrolysis product of the commonly used fluorogenic substrates 4-methylumbelliferyl (MUF)-phosphate, MUF-butyrate, MUF-alpha-D-glucose and methyl-coumarinyl-amide(MCA)-leucine (SigmaAldrich) over time (Hoppe, 1993).Seawater from each sample was aliquoted into 96-well plate in triplicate. The fluorogenic substrate was added to a final concentration of $25 \mu \mathrm{M}$ (Hmelo et al., 2011) and the kinetics of fluorescence production was measured using a CytoFluor Series 4000 multi-well plate reader. Fluorescence was converted to concentration units using calibration curves of commercially available, purified MUF and MCA standards. Technical replicates were averaged for each triplicate treatment to avoid pseudo-replication. 


\subsection{Cell Enumeration}

A $750 \mu \mathrm{l}$ sample from each BOD bottle was preserved in $1 \%$ formaldehyde. The cells were allowed to fix at room temperature for approximately 30 minutes before flash freezing in liquid nitrogen and storing in a $-80{ }^{\circ} \mathrm{C}$ freezer. The samples were transported back to the lab in a dry shipper. In the lab, the samples were thawed, vortexed, and then diluted with TE buffer by 100 -fold, as the flow cytometer used can only reliably enumerate cells up to $2.5 \times 10^{5}$ cells ml $^{-1}$. Triplicates of each sample were pipetted into a 96-well plate and stained with SYBR-green (Tripp, 2008). The cells were enumerated using a 5H Guava EasyCyte flow cytometer. Blanks of TE buffer were also enumerated and used to correct for cells added during dilution. The technical replicates were average for each triplicate treatment to avoid pseudo-replication.

\subsection{Estimation of the contribution of free-living vs. particle-associated bacteria in the incubation experiments.}

Incubation experiments were initiated by mixing seawater that had been filtered through a $0.2 \mu \mathrm{m}$ poresize membrane with slurries of sinking particles captured by net traps. We found that the filtration reduced the concentration of free-living bacteria by about $95 \%$; in the Sargasso Sea, concentrations were reduced from an average of $1.9 \times 10^{5}$ cell ml${ }^{-1}$ to an average of $1.1 \mathrm{x}$ $10^{4}$ cell $\mathrm{mL}^{-1}$. Generally, the ratio of filtered seawater to particle slurries was $1: 1$. The average cell concentration of the incubation at $\mathrm{t}=$ initial was $1.0 \times 10^{6}$ cell $\mathrm{mL}^{-1}$. The below calculation indicates that particle-associated bacteria comprised $>99 \%$ of the bacteria in our $150 \mathrm{~mL}$ incubations.

(Concentration of free cells in filtered seawater $\times$ Volume added)

+ (Concentration of particle associated cells in trap slurry

$\times$ Volume added $)=$ Concentration of cells in incubations at $t$ $=$ initial $\times$ Total Volume of incubations

$\left(1.0 \times 10^{4}\right.$ cells $\left.m L^{-1}\right) \times(75 m L)$

+ (Concentration of particle associated cells in trap slurry) $\times 75 \mathrm{~mL}$ $=\left(1.0 \times 10^{6}\right.$ cells $\left.\mathrm{mL}^{-1}\right) \times(150 \mathrm{~mL})$

Concentration of particle associated cells in trap slurry $=1.99 \times 10^{6}$ cells $\mathrm{mL}^{-1}$ 
In other words, of the $1.5 \times 10^{8}$ cells in each incubation, $1.49 \times 10^{8}$ were introduced into the incubations via the slurry of particles. We attribute the responses observed in the incubations to the dominant particle-associated community, even if they sloughed off during the course of incubations. An alternative explanation that free-living bacteria were orders of more active than the particle-associated community cannot be absolutely precluded. However, cell specific activity rates of particle associated bacteria are generally thought to be much more active than free-living, not the other way around (Smith DC, 1992). Furthermore, amendments of nanomolar concentrations of PUAs (versus the micromolar concentrations used in the present study), led to reductions in the cell-specific activities of natural communities of free-living marine bacteria (Balestra et al., 2011). Thus attributing the observed responses (Fig 3, Fig 4, and Fig. 4) to the particle-attached bacteria is more consistent with existing knowledge than the alternative explanation.

\subsection{Bacterial Production}

Bacterial production rates within triplicate incubations for each of the PUA treatments at TWNA1, TWNA2, and SS3 were determined by tracing the uptake of ${ }^{3} \mathrm{H}$-leucine using the standard microcentrifuge method (Smith and Azam, 1992). Briefly, $1.5 \mathrm{~mL}$ aliquots from the incubations were collected in microcentrifuge tubes and spiked with L-[3,4,5- $\left.{ }^{3} \mathrm{H}(\mathrm{N})\right]-\mathrm{Leucine}$ to a final concentration of $20 \mathrm{nmol} \mathrm{L}^{-1}$. Samples were then place in the dark at in situ temperatures, which were the same temperatures as the incubations, for approximately four hours. Next $80 \mu \mathrm{L}$ of $100 \%$ trichloroacetic acid (TCA) was added to the tubes, which were then vortexed and placed on ice. The tubes were centrifuged at approximately 15,000 x G. The resultant pellet of precipitated proteins (and other macromolecules) was further purified by rinsing/centrifuging with ice-cold 5\% TCA and $80 \%$ ethanol. Finally, $1 \mathrm{~mL}$ of scintillation cocktail was added to the tube, and the quench-corrected ${ }^{3} \mathrm{H}$ radioactivity was determined by liquid scintillation counting.

\subsection{PUA extraction}

At the end of the incubation period, a $20 \mathrm{~mL}$ sample of each triplicate was taken for PUA derivatization and extraction at sea. For experiments SS3, TWNA1, TWNA2, and SANA1, $\mathrm{t}=$ initial samples were also extracted. Benzaldehyde was added, as an internal standard, to a final concentration of $10 \mu \mathrm{M}$. The aldehydes within the samples were derivatized by addition of 
DNPH dissolved in $1 \%$ sulfuric acid (final DNPH concentration $200 \mu \mathrm{M}$ ). The derivatization reaction was allowed to proceed at room temperature for 15 minutes, vortexing every 5 minutes. Sulfuric acid is a known protein denaturant, precluding biological production of PUAs in response to vortexing or subsequent sample handling. The reaction was sonicated for 3 minutes before adding $10 \mathrm{ml}$ of hexane. The organic extraction was allowed to take place over 15 minutes, continuing to vortex every 5 minutes. After another 3 minute round of sonication, the aqueous phase was removed. The aldehyde extract was placed in a $55{ }^{\circ} \mathrm{C}$ water bath and blown down under $\mathrm{N}_{2}$ gas to a volume of approximately $1 \mathrm{ml}$. The aldehyde extract was transferred to a $2 \mathrm{ml}$ HPLC vial and dried down in a vaccufuge. The samples were then capped under $\mathrm{N}_{2}$ and stored at $-80{ }^{\circ} \mathrm{C}$. The samples were flown back to the lab in a dry shipper and immediately redissolved in 70:30 acetonitrile:water.

\subsection{PUA quantification}

PUAs were quantified using high performance liquid chromatography (HPLC) coupled with UV-visible spectroscopy using a photodiode-array detector (Thermo Finnigan) monitoring the $360 \mathrm{~nm}$ channel. An isocratic chromatographic method was performed on an Agilent 1200 HPLC system (Agilent Technologies) with 73\% acetonitrile and 27\% 70:30 acetonitrile:water as the mobile phase. An Agilent C18 column (Zorbax ODS 5 $\mu \mathrm{m} 3.0 x 150 \mathrm{~mm}$ ) served as the stationary phase. Atmospheric pressure chemical ionization (APCI)- triple quadrupole mass spectrometry (TQMS) was used to verify that the peaks detected with HPLC-UV vis spectroscopy were indeed the molecules of interest. The mass spectrometer used was a Thermo TSQ Vantage TQMS operating in the negative ionization mode. A calibration curve was constructed for dilutions of a mixed PUA standard containing DNPH-derivatized trans, trans-2,4heptadienal (Sigma-Aldrich), trans,trans-2,4-octadienal (Sigma Aldrich), trans, trans-2,4decadienal (Acros Organics), and benzaldhyde (Sigma Aldrich). The internal standard DNPHbenzaldehyde eluted at $\sim 1.3$ minutes. DNPH-heptadienal eluted at $\sim 1.6$ minutes with a negative ion $\mathrm{m} / \mathrm{z}$ of 288.9. DNPH-octadienal eluted at $\sim 1.9$ minutes with a negative ion $\mathrm{m} / \mathrm{z}$ of 302.8 . DNPH-decadienal eluted at $~ 3.0$ minutes with a negative ion $\mathrm{m} / \mathrm{z}$ of 330.9. The $\mathrm{MS}^{2}$ spectra for each chromatographic peak were further analyzed. The identity of each compound was confirmed by the characteristic fragmentation of unsaturated aldehydes ((Kolliker et al., 1998); Fig. 2). 
We tested the hypothesis that the $\mathrm{O}_{2}$ consumption via respiration in our experiments $(\Delta$ $\left.\left[\mathrm{O}_{2}\right]=\left[\mathrm{O}_{2}\right]_{\mathrm{t}=\text { initial }}-\left[\mathrm{O}_{2}\right]_{\mathrm{t}=\text { final }}\right)$ was not supported solely by the respiration of exogenous PUAs. To do this, we converted the observed changes in PUA concentrations $\left(\Delta[\mathrm{PUA}]=[\mathrm{PUA}]_{\mathrm{t}=\text { initial }}-\right.$ $\left.[\mathrm{PUA}]_{\mathrm{t}=\text { final }}\right)$ to oxygen equivalent units $\left(\Delta\left[\mathrm{PUA}_{\mathrm{O} 2 \text { equivalents }}\right]\right)$ by calculating the $\Delta[\mathrm{PUA}]$ over the course of incubation for each PUA chain-length separately, using stoichiometric relationships to convert to moles $\mathrm{O}_{2}$, and then summing.

$$
\begin{aligned}
& \text { Test: } \Delta\left[\mathrm{O}_{2}\right]>\Delta\left[\mathrm{PUA}_{\mathrm{O} 2 \text { equivalents }}\right] \\
& \text { Heptadienal } \mathrm{C}_{7} \mathrm{H}_{10} \mathrm{O}+9 \mathrm{O}_{2} \rightarrow 7 \mathrm{CO}_{2}+5 \mathrm{H}_{2} \mathrm{O} ; \Delta\left[\mathrm{C} 7-\mathrm{PUA}_{\mathrm{O} 2 \text { equivalents }}\right]=9 \cdot \Delta[\mathrm{C} 7-\mathrm{PUA}] \\
& \text { Octadienal } \mathrm{C}_{8} \mathrm{H}_{12} \mathrm{O}+10.5 \mathrm{O}_{2} \rightarrow 8 \mathrm{CO}_{2}+6 \mathrm{H}_{2} \mathrm{O} ; \Delta\left[\mathrm{C} 8 \text { - } \mathrm{PUA}_{\mathrm{O} 2 \text { equivalents }}\right]=10.5 \cdot \Delta[\mathrm{C} 8 \text {-PUA] } \\
& \text { Decadienal } \mathrm{C}_{10} \mathrm{H}_{14} \mathrm{O}+13.5 \mathrm{O}_{2} \rightarrow 10 \mathrm{CO}_{2}+7 \mathrm{H}_{2} \mathrm{O} ; \Delta\left[\mathrm{C} 10-\mathrm{PUA}_{\mathrm{O} 2 \text { equivalents }}\right]=13.5 \cdot \Delta[\mathrm{C} 10 \text {-PUA }] \\
& \Delta\left[\mathrm{PUA}_{\mathrm{O} 2 \text { equivalents }}\right]=\Delta\left[\mathrm{C} 7-\mathrm{PUA}_{\mathrm{O} 2 \text { equivalents }}\right]+\Delta\left[\mathrm{C} 8-\mathrm{PUA}_{\mathrm{O} 2 \text { equivalents }}\right]+\Delta\left[\mathrm{C} 10-\mathrm{PUA}_{\mathrm{O} 2 \text { equivalents }}\right]
\end{aligned}
$$

The comparison between the average $\Delta\left[\mathrm{O}_{2}\right]$ the average $\Delta$ [PUA $\left.\mathrm{P}_{\mathrm{O} 2 \text { equivalents }}\right]$ was conducted for stations TWNA1, TWNA2, and SS3, which were the stations where we had obtained all of the necessary data (Table 4).

\subsubsection{Estimation of PUA concentrations within sinking particles}

As described above, incubations containing sinking particles were extracted using a liquid:liquid extraction protocol and the PUAs were quantified using UV-vis spectrometry. Then the concentrations of PUAs within sinking particles were estimated using the POC concentration in the incubations paired with a previously published relationship between POC and volume on diatom-derived marine snow particles (Brzezinski et al., 1997). This estimation assumes that PUAs are evenly distributed throughout the particulate organic matter and it effectively treats the organic matter in the $20 \mathrm{~mL}$ aliquot of incubation that was analyzed for PUAs as one large particle (Table 2). For the $\mathrm{t}=$ final incubations, these values are likely an underestimation as the POC content after 24 hours would be lower than the original trap material. 


$$
\frac{\text { mol PUA }}{L_{\text {incubation }}} \div \frac{\text { mol POC }}{L_{\text {incubation }}} \times \frac{\text { mol POC }^{\dagger}}{L_{\text {particle }}}=\frac{\text { mol PUA }}{L_{\text {particle }}}
$$

$\dagger$ From Brzezinski et al (1997).

Within these incubations, total endogenous PUA carbon represents between $0.6 \%$ and $5.4 \%$ of POC (Table 2), which supports our assertion that PUA are a small component of the organic carbon in sinking particles. For comparison, we used diatom physiology studies to estimate how much carbon within sinking particles might be expected to be attributable to PUAs. In pure cultures of the diatom Skeletonema marinoi, the production of PUAs from disrupted biomass reached $28 \mathrm{fmol} \mathrm{cell}^{-1}$ under silica-limited conditions(Ribalet et al., 2009); applying octadienal molecular weight $\left(124 \mathrm{~g} \mathrm{~mol}^{-1}\right)$ and molecular carbon content (77\% by mass), this is equivalent to $2.7 \mathrm{pg} \mathrm{C}$ cell $^{-1}$. Given that Si-limited cultures of $S$. marinoi have been shown to contain $17 \mathrm{pg} \mathrm{C}$ cell $^{-1}$ (Ribalet et al., 2009), PUAs could compose as much as $16 \%$ of the carbon in cell. By contrast, in bloom-forming field populations of S. marinoi, PUAs composed as much as approximately $1 \%$ of the carbon in wounded cells (Taylor, 2009) (again, assuming octadienal carbon content). Therefore, our results fall within the low end of the range of these two estimates of the fraction of carbon composed by PUAs, which makes sense since not all of the POC in our sediment trap samples was derived from diatoms.

\subsection{Particulate Organic Carbon content of trap material}

A $500 \mathrm{~mL}$ split of the trap material was filtered onto pre-combusted $0.7 \mu \mathrm{m}$ glass fiber filters for particulate organic carbon (POC) analysis. The samples were kept at $-80{ }^{\circ} \mathrm{C}$ until analysis. Back in the lab the filters were acidified (Whiteside et al., 2011) and then analyzed on a Finnigan-MAT DeltaPlus stable light isotope ratio mass spectrometer.

\subsection{Genomic DNA Extraction}

Samples for DNA were taken by filtering $50 \mathrm{ml}$ of each incubation onto a $25 \mathrm{~mm}$ Durapore filter. Each filter was placed in a cryovial and frozen at $-80{ }^{\circ} \mathrm{C}$ until extraction. Genomic DNA was extracted using a modified protocol and the Qiagen Blood and Tissue Kit (Santoro et al., 2010). The extracted DNA was quantified using Qubit dsDNA Broad Spectrum Kit (Life Technologies). Samples were stored at $-20{ }^{\circ} \mathrm{C}$ until amplification with PCR. 


\subsection{ARISA}

The intergenic spacer region (ITS) between the 16S and 23S rRNA genes was amplified by PCR with universal forward primer 1392F (5'-G[C/T]ACACACCGCCCGT-3') and bacterial reverse primer 23s-125R(5'-GGGTT[C/G/T]CCCCATTC(A/G)G-3') labeled with a 6-FAM. The $25 \mu 1$ reaction mixtures contained final concentrations of 1 X PCR buffer (Promega), 2.5 $\mathrm{mM} \mathrm{MgCl} 2$ (Promega), $250 \mu \mathrm{M}$ of each dNTP (Promega), $200 \mathrm{nM}$ of each primer, $40 \mathrm{ng} \mathrm{l}^{-1}$

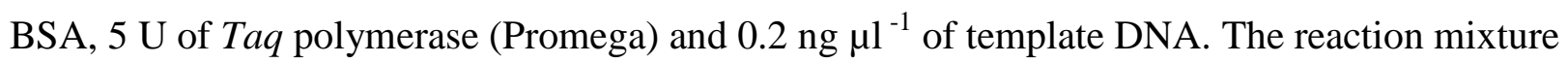
was held at $94{ }^{\circ} \mathrm{C}$ for $2 \mathrm{~min}$ followed by 35 cycles of amplification $\left(94{ }^{\circ} \mathrm{C}\right.$ for $40 \mathrm{~s}, 56{ }^{\circ} \mathrm{C}$ for $40 \mathrm{~s}$ and $72{ }^{\circ} \mathrm{C}$ for $90 \mathrm{~s}$ ), and a final step of $72{ }^{\circ} \mathrm{C}$ for $5 \mathrm{~min}$. PCR products were purified using Qiagen MinElute PCR purification columns. The purified DNA extracts were quantified using Qubit

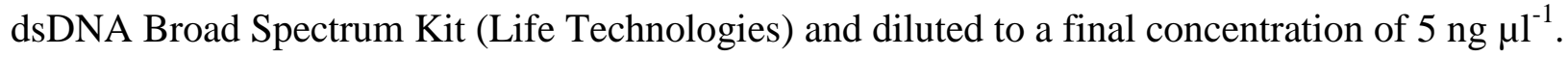
1ul aliquots were transferred to a 96 well plate and shipped on dry ice to the Core Molecular Biology lab at UMass Medical (Dartmouth, MA). There they were analyzed with an ABI GeneAnalyzer. Electropherograms were further analyzed at Woods Hole with ABI Peak Scanner v2. While a sample from each triplicate was sent off for ARISA ( $n=6)$, there were technical errors and three of the samples failed to be analyzed (a $10 \mu \mathrm{M}$ triplicate from TWNA1, a $10 \mu \mathrm{M}$ triplicate from SS3, and a $100 \mu \mathrm{M}$ triplicate from SS3). OTUs were assigned for ITS fragments between 400 and 1000bp. Peaks were binned according to fragment length: 400-450 bp $\pm 0.5 \mathrm{bp}$ bins, $450-650 \mathrm{bp} \pm 1$ bp bins, $650-900 \mathrm{bp} \pm 1.5$ bp bins, and $900-1400 \mathrm{bp} \pm 2.5$ bp bins (Needham et al., 2013). Multidimensional Scaling analysis was run on presence/absence of OTUs in STATISTICA. OTUs were assigned putative taxonomic identities using an ARISA ITS fragment length database compiled by Jed Fuhrman's lab group at USC that combined ITS region clone library sequence data from the USC Microbial Observatory, the Global Ocean Survey, in silico Cyanobacterial estimations, Biocomplexity project, Whole Genome project, and JCVI-Indian Ocean project. The method used for assigning identity is described in Needham et al., (2013).

\subsection{Statistical analysis}

In order to remove between-station variability and compare the incubation experiment data from all six stations, respiration rates, bacterial cell abundance, bacterial production, and enzymatic activity data were normalized to the data from the control incubation. To do this, the 
values from each incubation at a given station were divided by the corresponding average value of the control triplicates from that station. Control-normalized values greater than one represent increases in respiration rate, bacterial cell abundance, bacterial production or enzyme activity compared to the control, whereas, control normalized values less than one represent decreases in these parameters. A series of Wilcoxon tests on ranks was used to determine whether the distributions of data from the incubations amended with 1, 10 or $100 \mu \mathrm{M}$ PUAs treatments were different from the distributions of the data from the no-amendment controls. Any tests yielding P-values less than or equal to 0.05 were considered to be significant, and were identified in the manuscript as such. All statistics were run in STATISTICA software.

\section{Results}

\subsection{Exposure to PUAs affects changes in the rates of sinking POC remineralization.}

We tested the linkages between PUAs and the rate of organic matter remineralization by particle-associated bacteria at six stations across the North Atlantic Ocean (Fig. 1; Table 1): three stations in the Sargasso Sea (SS), two in the temperate western North Atlantic (TWNA), and one in the Subarctic North Atlantic (SANA). Our experimental methods centered on collecting sinking particles, incubating particles in the presence of exogenous PUAs (cocktail of heptadienal, octadienal, and decadienal) at a range of concentrations, and assessing changes in organic matter respiration, hydrolytic enzyme activity, bacterial cell abundance, bacterial production rates, and bacterial community structure. The absolute values of these parameters varied considerably between stations (Fig. 3). To remove between-stations variability we divided the average values of the PUA-amended treatments by the average of the no-amendment controls from each corresponding station. These control-normalized data showed strikingly similar responses by particle-associated bacteria to PUA treatments across this ocean basin (Fig 4 and 5). We then asked whether there were differences between the controls and the incubations amended with different concentrations of PUAs using a series of Wilcoxon ranked sum statistical tests.

In general, the addition of exogenous PUAs at lower concentrations led to stimulated rates of bacterial organic matter remineralization. The average respiration rates in 1 and $10 \mu \mathrm{M}$ treatments were approximately double that observed in the control treatment (Fig. 4). The average respiration rate in the $100 \mu \mathrm{M}$ treatment did not differ from the control. Enzyme activity 
assays revealed enhanced alkaline phosphatase (APase) and lipase activity compared to the control over the same stimulatory range of concentrations observed for respiration rates (Fig. 5, Fig 4). Average APase activity in the 1 and $10 \mu \mathrm{M}$ treatments was quadruple that of the controls. The average lipase activity was one and a half times that of the control in the 1 and 10 $\mu \mathrm{M}$ treatments. Lipase activity was significantly lower than the control in the $100 \mu \mathrm{M}$ treatments. Peptidase activity was significantly lower than the control in the 10 and $100 \mu \mathrm{M}$ treatments. Whereas alpha-glucosidase activity did not significantly deviate from the control in any of the treatments.

The changes in the rates of organic matter remineralization in response to PUAs were reflected in the growth of particle-associated bacteria. Bacterial cell abundances in the $10 \mu \mathrm{M}$ treatments were approximately $50 \%$ greater than in the controls (Fig. 4), whereas bacterial production was about $20 \%$ higher (Fig. 4). Similar responses in these signals, although of lesser magnitude, were observed in the $1 \mu \mathrm{M}$ PUA treatments, hinting at a dose-dependent growth response to PUAs. In contrast to the 1 and $10 \mu \mathrm{M}$ treatments, bacterial cell abundance was significantly lower than the control in the $100 \mu \mathrm{M}$ PUA treatments, pointing to an inhibitory threshold between 10 and $100 \mu \mathrm{M}$. The average bacterial production rates were also generally lower in the $100 \mu \mathrm{M}$ treatment compared to the control, but this effect was not statistically significant because of geographic variability; the two TWNA sites showed almost complete inhibition of bacterial production, whereas, SS3 showed stimulation (Fig. 3).

\subsection{Sinking particles are hotspots for PUA production}

Sinking particles were also collected for PUA analysis by HPLC-UV-MS ${ }^{\mathrm{n}}$. Decadienal was clearly observed in the sinking particles at TWNA2 prior to incubation (i.e. $\mathrm{t}=$ initial; Fig. 6); based on POC content of these sinking particles and a previously published relationship between POC and volume of diatom-derived marine snow particles (Brzezinski et al., 1997) the in situ concentration of decadienal within sinking particles was estimated to be $26 \mu \mathrm{M}$ (Fig. 7; Table 2 ). This concentration was comparable to the stimulatory range of concentrations observed in our incubation experiments. Additionally, significant production of PUAs of multiple chain lengths was observed in the no-amendment controls after 24 hours of incubation (i.e. $\mathrm{t}=$ final) in all three sampling regions (Fig. 7; Table 2); heptadienal, octadienal, and decadienal reached concentrations as high as $10.4,12.9$, and $34.0 \mu \mathrm{M}$, respectively. 


\subsection{Shift in bacterial community upon exposure to PUAs}

Changes in the community structure of particle-associated bacteria were assessed by using Automated Ribosomal Intergenic Spacer Analysis (ARISA) at one station in the TWNA (TWNA1) and at one station in the Sargasso Sea (SS3). The presence/absence of each OTU identified in the various treatments was analyzed with Multidimensional Scaling to describe the variations in community structure. The particle-associated bacteria communities from the control, $1 \mu \mathrm{M}$ and $10 \mu \mathrm{M}$ treatments formed two distinct clusters based on geographic location (Fig. 8), indicative of negligible impacts on bacterial community structure at these low PUA concentrations. In contrast, the $100 \mu \mathrm{M}$ treatments led to dramatic changes in community structure versus the other treatments.

An ARISA clone library database was used to assign a putative identity to each OTU and we calculated the relative abundance of the following bacterial phyla/classes: Actinobacteria, Bacteroidetes, Cyanobacteria, Deferribacteres, Firmicutes, $\alpha$-proteobacteria, $\beta$-proteobacteria, $\delta$ proteobacteria, $\mathcal{E}$-proteobacteria, and $\gamma$-proteobacteria (Fig. 9). The effects of PUAs on individual clades were assessed using Spearman's rank correlations. Abundances of most groups did not show a significant correlation with the amount of PUA added, which includes noted particle specialists (eg. Bacteriodetes and Firmicutes) (Crespo et al., 2013; DeLong et al., 1993). At the same time, $\gamma$-proteobacteria were significantly negatively correlated with PUA concentrations and Actinobacteria were significantly positively correlated (Table 3).

\section{Discussion}

Doses of PUAs ranging from 1 to $10 \mu \mathrm{M}$ stimulated organic matter respiration on sinking particles (Fig. 4). Enhanced organic matter respiration in these treatments supported bacterial growth, as suggested by parallel increases in bacterial cell abundance and bacterial production (Fig 4). Stimulatory concentrations of 1 to $10 \mu \mathrm{M}$ agree with data reported for two cultured bacterial strains, Eudora adriatica and Alteromonas hispanica, which showed enhanced growth rates when exposed to PUA concentrations as low as $13 \mu \mathrm{M}$ (Ribalet et al., 2008). Ribalet et al. (2008) conducted incubations that suggested that the bioactivity of PUAs is derived specifically from their combination of carbonyl group and double bonds, precluding the potential for PUAs to be used as a food source. 
If PUAs were a consistent food source, then a relationship between the PUA consumption $\left([\mathrm{PUA}]_{t=\text { initial }}-[\mathrm{PUA}]_{\mathrm{t}=\text { final }}\right)$ and oxygen consumption by respiration $\left((\mathrm{al} .)_{\mathrm{t}=\text { initial }}-(\mathrm{al} .)_{\mathrm{t}=\text { final }}\right)$ would be expected. This comparison was made for the 1 to $10 \mu \mathrm{M}$ treatments in experiments conducted at TWNA1, TWNA2, and SS3 where $[\mathrm{PUA}]_{t=\text { initial }}$ and $[\mathrm{PUA}]_{t=\text { final }}$ data were available (Table S4). In three of the six comparisons, the consumption of oxygen was greater than the drawdown in PUAs, yielding strong evidence in those instances that the stimulation of respiration was not driven solely by respiration of the PUA amendments themselves. Indeed, in the $10 \mu \mathrm{M}$ experiments at SS3, and in most of the control experiments across the study (Fig 3), net PUA production was observed, which further suggests that PUAs are not a readily accessible food source. The remaining two incubations where oxygen consumption was less than PUA drawdown were from TWNA1 and do not necessarily contradict the results from the other four incubations because PUAs could have been partially degraded, which would remove them from our analytical window without incurring stoichiometric oxygen consumption. The inconsistent relationship between respiration and PUA consumption bolsters our interpretation that the stimulatory effect of PUAs was not simply the result of direct respiration of these molecules.

Our data and subsequent calculations suggest that PUA concentrations in environmental samples of sinking particles were in the low micromolar range and comparable to the stimulatory range in the incubation experiments (Fig. 7; Table 2). The concentrations of PUAs within sinking particles were calculated using a previously published POC-volume relationship for diatom-derived marine snow particles (Brzezinski et al., 1997), and thus there are considerable uncertainties in these concentrations. However, dissolved concentrations of PUAs from phytoplankton in North Atlantic seawater were recently determined to be generally less than 1 picomolar (Bartual and al, 2014). Since the concentrations we observed in sinking particles are orders of magnitude higher, we propose that sinking particles are hotspots for PUA production. This idea is supported both by the direct observation of decadienal in native (i.e. $t=$ initial) particles from TWNA, as well as the accumulation of PUAs to micromolar concentrations within particles from four of the six no-amendment control treatments $(t=$ final; Fig. 7).

The accumulation of PUAs in the incubations also suggests that PUAs continue to be produced as particles descend into the mesopelagic. It will be important to quantify PUAs on particles at different depths (vs. only $150 \mathrm{~m}$ in this study) because the accumulation of PUAs to concentrations above $\sim 10 \mu \mathrm{M}$ during transit could potentially result in a transition between 
stimulatory and toxic effects on bacteria associated with the particles at particular depth horizons (Fig. 4). For example, a recent large-scale study of a diatom bloom in the North Atlantic concluded that export efficiency was low at depths above $100 \mathrm{~m}$ (al., 2012), but then increased substantially at depths below $100 \mathrm{~m}$ (Martin P, 2011); these results are consistent with our data suggesting that PUA dynamics could have complex and potentially contrasting effects on the export depths and the biogeochemical fate of POC sinking through the water column.

Export of POC to depth via sinking particles represents a globally significant carbon sink. The observed stimulatory concentrations of PUAs within sinking particles could affect biogeochemical cycling by decreasing POC export efficiency (Fig. 10). Direct PUA-enhanced respiration of sinking POC would accelerate the transfer of carbon from the organic carbon pool to the dissolved inorganic carbon pool; to a first approximation, the shallower this occurs the shorter the timescales of carbon sequestration from the atmosphere (Kwon et al., 2009). By increasing the hydrolysis of POC, stimulatory concentrations of PUAs could also cause additional transfer of carbon from the POC pool into the DOC pool through disaggregation or dissolution. Disaggregation leads to decreased sinking speeds and dissolution to greater rates of microbial utilization (Cochran et al., 1993; Smith DC, 1992). All of these PUA-induced changes cumulatively lead to shallower remineralization depths causing the release of $\mathrm{CO}_{2}$ in waters that are more likely to be mixed to the surface and re-equilibrate with the atmosphere on shorter timescales, thus attenuating carbon sequestration in the deep sea.

The shoaling of remineralization depths by PUAs would also affect greater release of inorganic nutrients from sinking particles in shallower waters (Fig. 5). The depth of mixing is 100-450 m in the North Atlantic during the winter (Michaels and Knap, 1996), and thus nutrients released from sinking particles above this depth during spring diatom blooms have the potential to fuel primary productivity in the subsequent spring. APase activity quadrupled in our incubation experiments suggesting PUAs led to enhanced release rates of dissolved inorganic phosphorus (Fig.2). Enhanced lipase activity directed toward phospholipids may also affect the liberation of dissolved phosphorus and other nutrients (Suzumura and Ingall, 2004). Phosphorus can be a limiting nutrient in regions of the North Atlantic (Ammerman et al., 2003). The C:P ratios of exported particles in these regions can be high, which could also point toward enhanced release of phosphorus (DeVries and Deutsch, 2014; Teng et al., 2014). Furthermore, physiological studies with diatoms in cultures and in mesocosm experiments have shown that 
PUA production increases under phosphorus-depleted conditions (Ribalet et al., 2009; Ribalet et al., 2007b). Thus there appear to be feedbacks between PUA production and organic phosphorus remineralization, potentially leading to the release of more phosphorus above the winter mixed layer, and thereby affecting greater rates of primary production on inter-annual and basin-wide scales.

While PUAs may stimulate the recycling of phosphorous, the decreases in peptidase activity (Fig 4) might indicate a decrease in biogenic Si remineralization on particles because diatom frustules are covered by glycoproteins that biochemically protect frustules from contact with seawater that is under-saturated in dissolved silica. Thus decreased peptidase activity directed toward these glycoproteins could result in lower dissolution of biogenic silica (BSi) in the euphotic zone (Bidle and Azam, 1999; KD et al., 2003), a process that supports $\sim 60 \%$ of global BSi production (Ragueneau et al., 2006). Preferential recycling of phosphorous over silica could play a role in phytoplankton community succession; in the North Atlantic, diatom blooms are followed by blooms of coccolithophores which do not have a silica requirement (Leblanc et al., 2009). It should be noted that the link between peptidase activity and BSi dissolution does not appear to be universal across all diatom-associated strains of bacteria (Bidle and Azam, 2001). Thus the impact of PUAs on BSi regeneration and the potential link with enhanced organic phosphorus cycling remain to be fully elucidated.

The variable response of different enzyme activities to different PUA concentrations might belie a connection between PUAs and the biochemical composition of sinking particles. Although the ectoenzymatic hydrolysis measurements were based on a few, well-established model substrates routinely used in microbial ecology since the 1980s (Hoppe, 1993), they likely do not encapsulate the complete enzymatic response to the molecularly diverse organic matter in sinking particles. Future studies could focus on how the addition of PUAs to sinking particles alters the biochemical composition of POC and DOC, as well as the exchange of classes of biochemicals between the various particulate and dissolved pools. Yet, our current data clearly show enhanced rates of respiration when particles were amended with ecologically relevant concentrations of PUAs. Notably, respiration represents the ultimate utilization and complete remineralization of organic matter to $\mathrm{CO}_{2}$ regardless of the biochemical composition of the particles or the enzyme activities of particle-associated bacteria. 
Our finding that the addition of high doses of PUAs significantly altered the community structure of particle-associated bacteria suggests that PUAs could play a role in bacterial community succession on sinking particles as PUA levels accumulate. Indeed, $100 \mu \mathrm{M}$ PUA treatments exhibited dramatic shifts in community structure combined with significant decreases in bacterial cell abundance (Fig 3 and Fig. 4). In contrast, lower levels of PUA amendments in the $1-10 \mu \mathrm{M}$ range stimulated bacterial metabolic activity (cell abundance, production, and APase activity) (Fig 3 and Fig 4), while affecting much more subtle shifts in resident bacterial community, which included phyla and classes known to contain particle specializers (Firmicutes and Bacteriodetes) (Fig. 4 and Fig. S4).

By using Spearman's rank correlations to assess the effects of PUAs on individual bacterial clades, we were able to identify one PUA-sensitive clade and one PUA-tolerant clade. The negative correlation between PUA addition and the relative abundance of $\gamma$-proteobacteria (Table S3), suggests that is group is PUA-sensitive. This is consistent with a report that $\gamma$ proteobacteria became dominant in the surface community in the North Sea only after a bloom of potentially PUA-rich diatoms subsided (Teeling, 2012). By contrast, the relative abundance of Actinobacteria was positively correlated with PUA addition, and this clade was previously shown to be strongly correlated with diatom pigments in the Sargasso Sea (Nelson et al., 2014). We found no other correlations between PUA amendments and any of the other clades we were able to resolve with ARISA. Ribalet et al. noted that bacterial isolates within the same genera had the potential to respond differently to PUAs (Ribalet et al., 2008), which suggests that PUA sensitivity is not strictly defined by taxonomic position.

In contrast to our $100 \mu \mathrm{M}$ results, recent work by Paul et al. concluded that PUAs do not impact the structure of free living bacterial communities (Paul and al, 2012). However, these investigators examined the effects of individual PUAs at nanomolar concentrations, and it is possible that nanomolar concentrations simply do not affect community structure, while micromolar concentrations do. In addition, it should be noted that since particle-associated and free-living bacterial communities are distinct (Bidle and Fletcher, 1995; DeLong et al., 1993), their corresponding stimulatory and inhibitory concentration ranges are also likely to be different (Amin et al., 2012; Ribalet et al., 2008). Alternatively, multiple chain lengths of PUAs together are often more potent than the same chain lengths separately, as has been observed for the metabolic activity of coastal free-living bacteria (Balestra et al., 2011). Diatoms often release 
multiple chain lengths of PUAs at once (Fontana et al., 2007) and the accumulation of heptadienal, octadienal, and decadienal in many of our no-amendment controls suggest that this is the case on sinking particles across the North Atlantic (Fig. 3).

The mechanisms by which PUAs stimulate or inhibit marine bacteria are not known. It has been proposed that PUAs stimulate bacteria by acting as growth co-factors (14). It is also possible that PUAs released from diatoms in sinking particles function as cues for the presence

of a larger pool of labile organic matter, which bacteria respond to with enhanced enzymatic and catabolic activity. We speculate that PUAs might be bona fide signals that diatoms secrete upon cell death in order to stimulate remineralization by bacteria, and thereby increase the likelihood that nutrients such as $\mathrm{P}$ are retained in surface water for the rest of the population to utilize (Fig. 5). On the other hand, PUAs are known Michael-acceptors, which are highly reactive toward $\mathrm{NH}_{2}$ and/or - $\mathrm{SH}$ groups, with the potential to cause unspecific damage inside bacterial cells (Adolph et al., 2004); Michael reactions could explain PUAs' inhibitory effects at high concentrations. Clearly additional work is necessary to understand the cellular and molecular bases for the impact of PUAs on marine bacteria in sinking particles.

\section{Conclusions}

This is one of the first reports showing that a specific class of bioactive molecules from phytoplankton impacts the activity and community structure of natural bacterial communities associated with sinking particles. We observed consistent dose-dependent bioactivity of PUAs in six iterations of the same incubation experiment across three different regions of the North Atlantic. Higher respiration rates, APase activity, lipase activity, and bacterial growth were observed over a stimulatory range of PUA exposure (1-10 $\mu \mathrm{M})$ and for generally similar bacterial communities. PUAs at higher concentrations tended to have inhibitory effects, and induced dramatic shifts in the bacterial community structure, demonstrating that PUAs may play a role in bacterial community succession on sinking particles. Decadienal concentrations comparable to the observed stimulatory range were observed within sinking particles collected in the TWNA and PUAs accumulated in incubations at other locations, suggesting that sinking particles are hotspots for PUA production.

Overall, the data are consistent with the hypothesis that PUAs in sinking particles affect an increase in remineralization of sinking particles, which results in a concomitant decrease in 
the efficiency of POC export from surface waters. This could in turn lead to retention of phosphorus and other nutrients in shallower waters, potentially fueling increased primary productivity on inter-annual timescales (Fig. 5). Although PUAs are only a very small component of the organic carbon in sinking particles (Table S2), their bioactivity exerts a disproportionate influence on the fate of this carbon in the mesopelagic zone. Our results support a broad-reaching "bioactivity hypothesis", which states that the bioactivity of the organic matter itself, through its ability to stimulate or inhibit particle-associated bacteria, affects POC export in much the same way that mineral protection and ballasting affect the efficiency of POC export (Armstrong et al., 2001; Francois et al., 2002; Klaas and Archer, 2002). Testing this hypothesis will involve spatially comprehensive field-based research focused on numerous molecular targets, efforts that must ultimately go far beyond our current study.

\section{References}

Adolph, S., Bach, S., Blondel, M., Cueff, A., Moreau, M., Pohnert, G., Poulet, S.A., Wichard, T., Zuccaro, A., 2004. Cytotoxicity of diatom-derived oxylipins in organisms belonging to different phyla, J Exp Biol, England, pp. 29352946.

Alkire, M.B., D'Asaro, E.D, Lee, C., Perry, M.J., Gray, A., Cetinic, I., Briggs, N., Rehm, E., Kallin, E., Kaiser, J., Gonzalez-Postada, A., 2012. - Estimates of net community production and export using high-resolution, Lagrangian measurements of O2, NO3-, and POC through the evolution of a spring diatom bloom in the North Atlantic. DeepSea Research I 64, 157-174.

Amin, S.A., Parker, M.S., Armbrust, E.V., 2012. Interactions between diatoms and bacteria. Microbiology and Molecular Biology Reviews 76, 667-684.

Ammerman, J.W., Hood, R.R., Case, D.A., Cotner, J.B., 2003. Phosphorus deficiency in the Atlantic: An emerging paradigm in oceanography. Eos, Transactions American Geophysical Union 84, 165-170.

Armstrong, R.A., Lee, C., Hedges, J.I., Honjo, S., Wakeham, S.G., 2001. A new, mechanistic model for organic carbon fluxes in the ocean based on the quantitative association of POC with ballast minerals. Deep Sea Research Part II: Topical Studies in Oceanography 49, 219-236.

Azam, F., Smith, D.C., Steward, G.F., Hagstrom, A., 1994. Bacteria-organic matter coupling and its significance for oceanic carbon cycling. Microbial Ecology 28, 167-179.

Balestra, C., Alonso-Saez, L., Gasol, J.M., Casotti, R., 2011. Group-specific effects on coastal bacterioplankton of polyunsaturated aldehydes produced by diatoms. Aquatic Microbial Ecology 63, 123-131.

Ban, S., et al., 1997. The paradox of diatom-copepod interactions. Marine Ecology Progress Series 157, $287-293$.

Bartual, A., al, e., 2014. Polyunsaturated Aldehydes from Large Phytoplankton of the Atlantic Ocean Surface (42 N to $33 \mathrm{~S}$ ). Marine drugs 12, 682-699. 
Bidle, K.D., Azam, F., 1999. Accelerated dissolution of diatom silica by marine bacterial assemblages. Nature 397, 508-512.

Bidle, K.D., Azam, F., 2001. Bacterial control of silicon regeneration from diatom detritus: significance of bacterial ectohydrolases and species identity. Limnology and Oceanography 46, 1606-1623.

Bidle, K.D., Fletcher, M., 1995. Comparison of free-living and particle-associated bacterial communities in the chesapeake bay by stable low-molecular-weight RNA analysis. Applied and environmental microbiology 61, 944952.

Brzezinski, M.A., Alldredge, A.L., O'Bryan, L.M., 1997. Silica cycling within marine snow. Limnology and Oceanography 42, 1706-1713.

Casotti, R., Mazza, S., Brunet, C., Vantrepotte, V., Ianora, A., Miralto, A., 2005. Growth inhibition and toxicity of the algal aldehyde 2-trans-2-cis decadienal on Thalassiosira weissflogii (Bacillariophyceae). Journal of Phycology 41, 7-20.

Cochran, J.K., Buesseler, K.O., Bacon, M.P., Livingston, H.D., 1993. Thorium isotopes as indicators of particle dynamics in the upper ocean: Results from the JGOFS North Atlantic Bloom Experiment. Deep Sea Research Part I: Oceanographic Research Papers 40, 1569-1595.

Crespo, B.G., Pommier, T., Fernández-Gómez, B., Pedrós-Alió, C., 2013. Taxonomic composition of the particleattached and free-living bacterial assemblages in the Northwest Mediterranean Sea analyzed by pyrosequencing of the 16S rRNA. MicrobiologyOpen 2, 541-552.

DeLong, E.F., Franks, D.G., Alldredge, A.L., 1993. Phylogenetic diversity of aggregate-attached vs. free-living marine bacterial assemblages. Limnology and Oceanography 38, 924-934.

DeVries, T., Deutsch, C., 2014. Large-scale variations in the stoichiometry of marine organic matter respiration. Nature Geosci 7, 890-894.

Edwards, B.R., Reddy, C.M., Camilli, R., Carmichael, C.A., Longnecker, K., Van Mooy, B.A.S., 2011. Rapid microbial respiration of oil from the Deepwater Horizon spill in offshore surface waters of the Gulf of Mexico. Environmental Research Letters 6, 035301.

Fontana, A., d'Ippolito, G., Cutignano, A., Miralto, A., Ianora, A., Romano, G., Cimino, G., 2007. Chemistry of oxylipin pathways in marine diatoms. Pure and Applied Chemistry 79, 481-490.

Francois, R., Honjo, S., Krishfield, R., Manganini, S., 2002. Factors controlling the flux of organic carbon to the bathypelagic zone of the ocean. Global Biogeochemical Cycles 16, 34-31-34-20.

Goutx, M., al, e., 2007. Composition and degradation of marine particles with different settling velocities in the northwestern Mediterranean Sea. Limnology and Oceanography 52, 1645.

Hmelo, L.R., Mincer, T.J., Van Mooy, B.A.S., 2011. Possible influence of bacterial quorum sensing on the hydrolysis of sinking particulate organic carbon in marine environments. Environmental microbiology reports 3 , 682-688.

Hoppe, H.G., 1993. Use of fluorogenic model substrates for extracellular enzyme activity (EEA) measurement of bacteria. Handbook of methods in aquatic microbial ecology, 423-431.

Ianora, A., Miralto, A., 2010. Toxigenic effects of diatoms on grazers, phytoplankton and other microbes: a review. Ecotoxicology 19, 493-511. 
Ianora, A., Poulet, S.A., 1993. Egg viability in the copepod Temora stylifera. Limnology and Oceanography 38, $1615-1626$.

Bidle, K.D., Brzezinski, M.A., Long, R.A., Jones, J.L., Azam, F., 2003. Diminished efficiency in the oceanic silica pump caused by bacteria-mediatedsilica dissolution. 48, 1855-1868.

Klaas, C., Archer, D.E., 2002. Association of sinking organic matter with various types of mineral ballast in the deep sea: Implications for the rain ratio. Global Biogeochemical Cycles 16, 63-61-63-14.

Kolliker, S., Oehme, M., Dye, C., 1998. Structure elucidation of 2, 4-dinitrophenylhydrazone derivatives of carbonyl compounds in ambient air by HPLC/MS and multiple MS/MS using atmospheric chemical ionization in the negative ion mode. Analytical chemistry 70, 1979-1985.

Kwon, E.Y., Primeau, F., Sarmiento, J.L., 2009. The impact of remineralization depth on the air-sea carbon balance. Nature Geoscience 2, 630-635.

Leblanc, K., Hare, C.E., Feng, Y., Berg, G.M., DiTullio, G.R., Neeley, A., Benner, I., Sprengel, C., Beck, A., Sanudo-Wilhelmy, S.A., Passow, U., Klinck, K., Rowe, J.M., Wilhelm, S.W., Brown, C.W., Hutchins, D.A., 2009. Distribution of calcifying and silicifying phytoplankton in relation to environmental and biogeochemical parameters during the late stages of the 2005 North East Atlantic Spring Bloom. Biogeosciences 6, 2155-2179.

Martin P, L.R., Perry MJ, Sanders R,, 2011. Export and mesopelagic particle flux during a North Atlantic spring diatom bloom. - 58, 338-349.

Michaels, A.F., Knap, A.H., 1996. Overview of the US JGOFS Bermuda Atlantic Time-series Study and the Hydrostation S program. Deep Sea Research Part II: Topical Studies in Oceanography 43, 157-198.

Miralto, A., Barone, G., Romano, G., Poulet, S.A., Ianora, A., Russo, G.L., Buttino, I., Mazzarella, G., Laabir, M., Cabrini, M., 1999. The insidious effect of diatoms on copepod reproduction. Nature 402, 173-176.

Needham, D.M., Chow, C.E.T., Cram, J.A., Sachdeva, R., Parada, A., Fuhrman, J.A., 2013. Short-term observations of marine bacterial and viral communities: patterns, connections and resilience. The ISME journal 7, 1274-1285.

Nelson, C.E., Carlson, C.A., Ewart, C.S., Halewood, E.R., 2014. Community differentiation and population enrichment of Sargasso Sea bacterioplankton in the euphotic zone of a mesoscale mode water eddy. Environmental microbiology 16, 871-887.

Paul, C., al, e., 2012. Diatom derived polyunsaturated aldehydes do not structure the planktonic microbial community in a mesocosm study. Marine drugs 10, 775-792.

Peterson, M.L., Wakeham, S.G., Lee, C., Askea, M.A., Miquel, J.C., 2005. Novel techniques for collection of sinking particles in the ocean and determining their settling rates. Limnology and Oceanography: Methods 3, 520532 .

Poulet, S.A., Laabir, M., Ianora, A., Miralto, A., 1995. Reproductive response of Calanus helgolandicus. I. Abnormal embryonic and naupliar development. Marine Ecology Progress Series 129, 85-95.

Ragueneau, O., Schultes, S., Bidle, K., Claquin, P., Moriceau, B., 2006. Si and C interactions in the world ocean: Importance of ecological processes and implications for the role of diatoms in the biological pump. Global Biogeochemical Cycles 20, GB4S02.

Ribalet, F., Berges, J.A., Ianora, A., Casotti, R., 2007a. Growth inhibition of cultured marine phytoplankton by toxic algal-derived polyunsaturated aldehydes. Aquatic Toxicology 85, 219-227. 
Ribalet, F., Intertaglia, L., Lebaron, P., Casotti, R., 2008. Differential effect of three polyunsaturated aldehydes on marine bacterial isolates. Aquatic Toxicology 86, 249-255.

Ribalet, F., Vidoudez, C., Cassin, D., Pohnert, G., Ianora, A., Miralto, A., Casotti, R., 2009. High Plasticity in the Production of Diatom-derived Polyunsaturated Aldehydes under Nutrient Limitation: Physiological and Ecological Implications. Protist 160, 444-451.

Ribalet, F., Wichard, T., Pohnert, G., Ianora, A., Miralto, A., Casotti, R., 2007b. Age and nutrient limitation enhance polyunsaturated aldehyde production in marine diatoms. Phytochemistry 68, 2059-2067.

Santoro, A.E., Casciotti, K.L., Francis, C.A., 2010. Activity, abundance and diversity of nitrifying archaea and bacteria in the central California Current. Environmental microbiology 12, 1989-2006.

Schilmiller, A.L., Howe, G.A., 2005. Systemic signaling in the wound response. Current Opinion In Plant Biology 8, 369-377.

Smith, D.C., Azam, F., 1992. A simple, economical method for measuring bacterial protein synthesis rates in seawater using 3H-leucine. Mar. Microb. Food Webs 6, 107-114.

Smith DC, S.M., Alldredge AL, Azam F, 1992. Intense hydrolytic enzyme activity on marine aggregates and implications for rapid particle dissolution. Nature 359, 10.

Smith, D.C., Steward, G.F., Long, R.A., Azam, F., 1995. Bacterial mediation of carbon fluxes during a diatom bloom in a mesocosm. Deep Sea Research Part II: Topical Studies in Oceanography 42, 75-97.

Suzumura, M., Ingall, E.D., 2004. Distribution and dynamics of various forms of phosphorus in seawater: insights from field observations in the Pacific Ocean and a laboratory experiment. Deep Sea Research Part I: Oceanographic Research Papers 51, 1113-1130.

Taylor, R.L., Abrahamsson, K., Godhe, A., Wangberg S., 2009. Seasonal Variability in Polyunsaturated Aldehyde Production Potential among Strains of Skeletonema Marinoi (Bacillariophyceae)1. Journal of Phycology 45, 46--53.

Teeling, H., 2012. Substrate-controlled succession of marine bacterioplankton populations induced by a phytoplankton bloom. Science 336, 608-611.

Teng, Y.-C., Primeau, F.W., Moore, J.K., Lomas, M.W., Martiny, A.C., 2014. Global-scale variations of the ratios of carbon to phosphorus in exported marine organic matter. Nature Geosci 7, 895-898.

Tripp, H.J., 2008. Counting marine microbes with Guava Easy-Cyte 96 well plate reading flow cytometer. Nature Protoc. Exch.

Van Mooy, B.A.S., Keil, R.G., Devol, A.H., 2002. Impact of suboxia on sinking particulate organic carbon: Enhanced carbon flux and preferential degradation of amino acids via denitrification. Geochimica et Cosmochimica Acta 66, 457-465.

Vidoudez, C., Casotti, R., Bastianini, M., Pohnert, G., 2011. Quantification of dissolved and particulate polyunsaturated aldehydes in the Adriatic Sea. Marine drugs 9, 500-513.

Wakeham, S.G., Hedges, J.I., Lee, C., Peterson, M.L., Hernes, P.J., 1997. Compositions and transport of lipid biomarkers through the water column and surficial sediments of the equatorial Pacific Ocean. Deep Sea Research Part II: Topical Studies in Oceanography 44, 2131-2162.

Whiteside, J.H., Olsen, P.E., Eglinton, T.I., Cornet, B., McDonald, N.G., Huber, P., 2011. Pangean great lake paleoecology on the cusp of the end-Triassic extinction. Palaeogeography, Palaeoclimatology, Palaeoecology 301, $1-17$. 
Wichard, T., Gerecht, A., Boersma, M., Poulet, S.A., Wiltshire, K., Pohnert, G., 2007. Lipid and fatty acid composition of diatoms revisited: rapid wound-activated change of food quality parameters influences herbivorous copepod reproductive success. ChemBioChem 8, 1146-1153. 


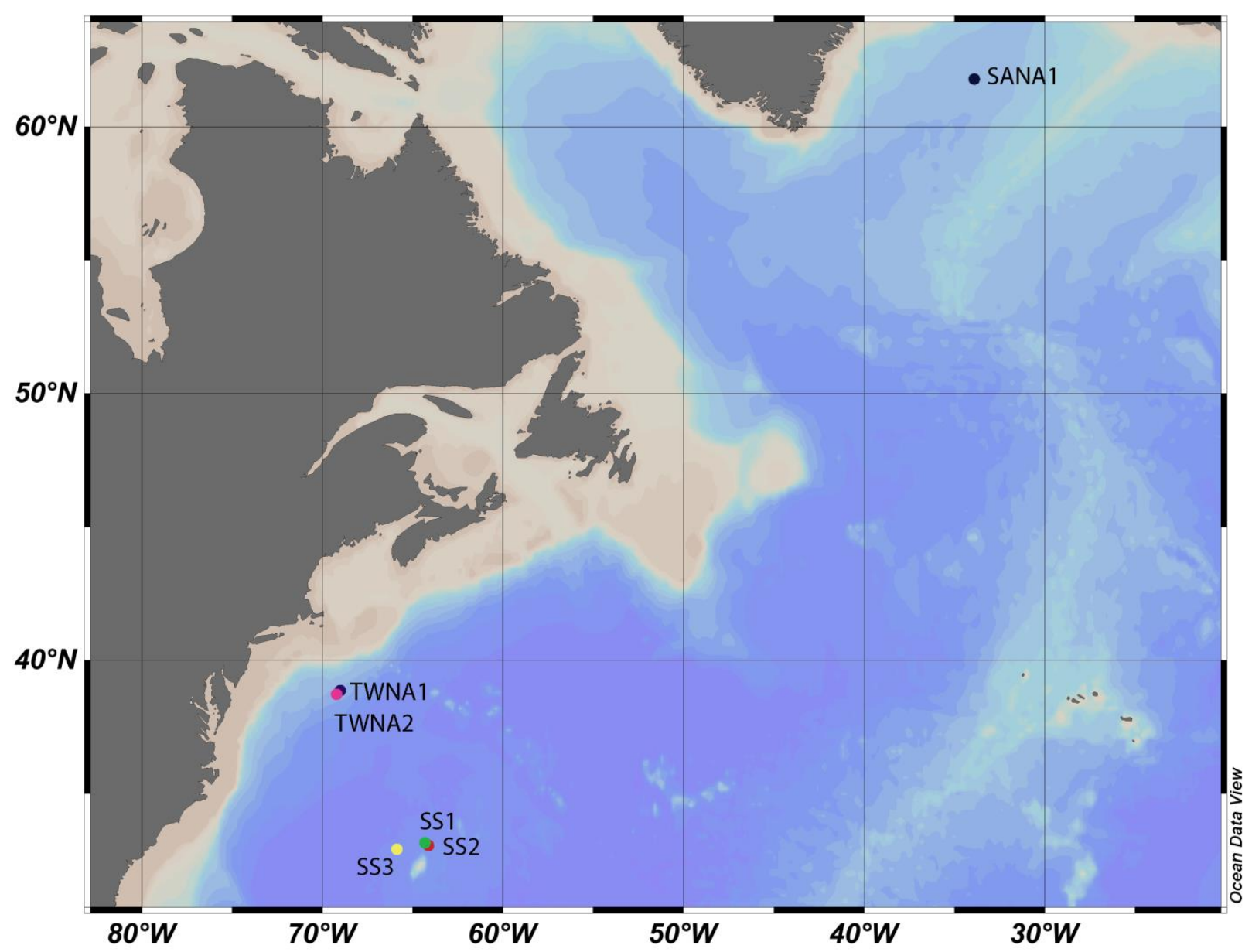

Figure 1. Figure S1. Map of the study sites covering three geographic regions: Temperate western North Atlantic (TWNA), the Sargasso Sea (SS), and the Subarctic North Atlantic (SANA). Each dot represents a trap recovery site: SS1-green, SS2-red, SS3-yellow, TWNA1purple, TWNA2-pink, and SANA1-black 

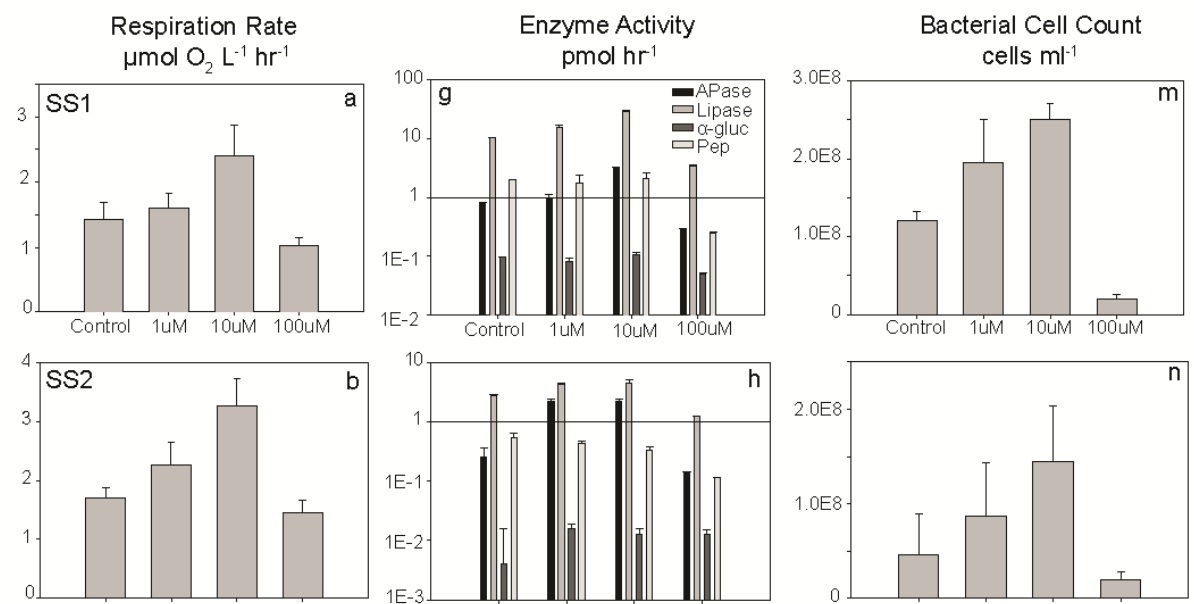

Bacterial Production $\mathrm{nmol} \mathrm{C} \mathrm{L-1} \mathrm{hr}^{-1}$
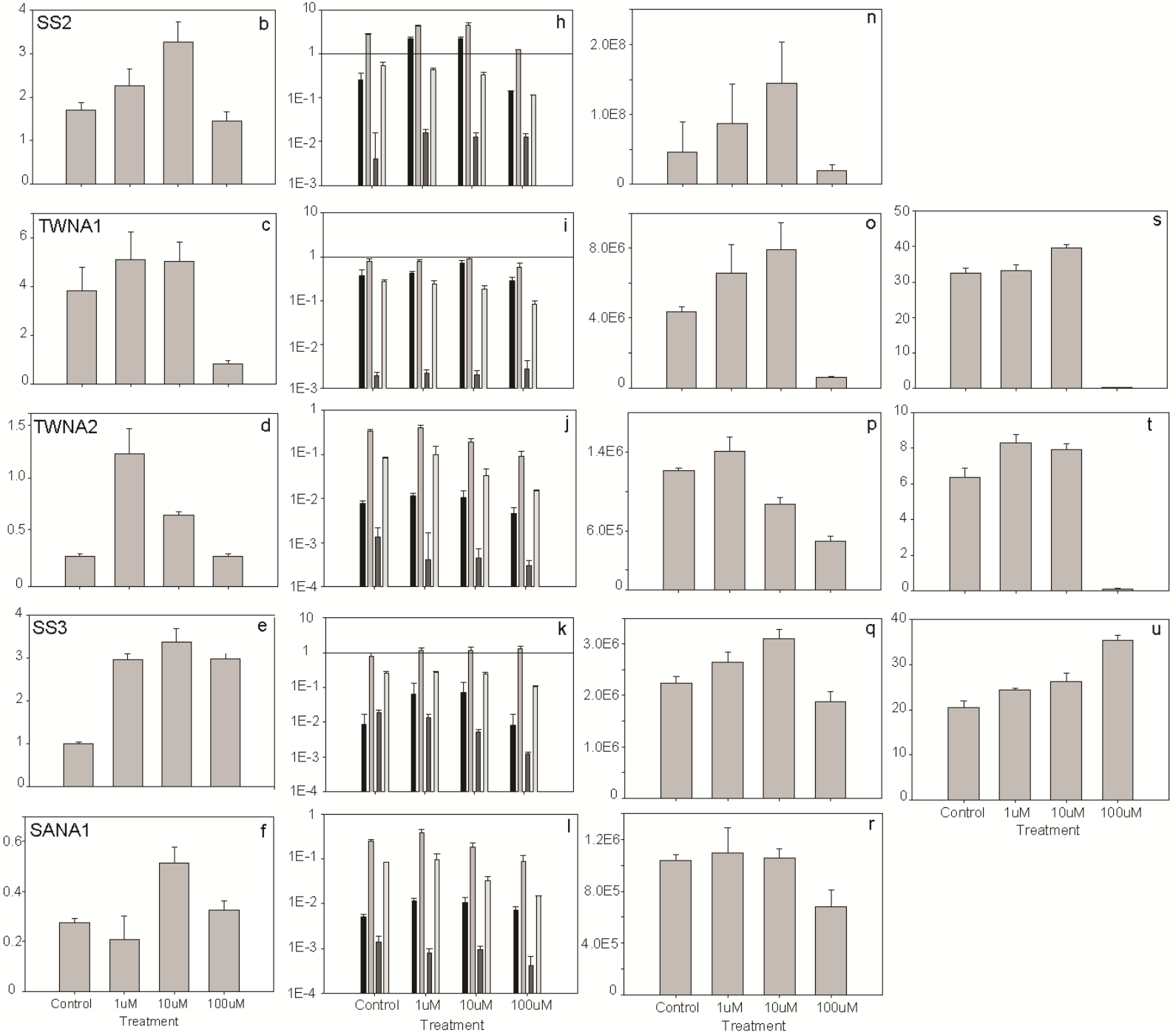

Figure 2. The response of the microbial community to PUAs in each experiment showing average values normalized to the respective quantities in control incubations for each parameter: a - f) respiration rate; $\mathrm{g}$ - l) ectohydrolytic enzymatic activities; $\mathrm{m}-\mathrm{r}$ ) bacterial cell abundance; $\mathrm{s}$ - u) bacterial production rate. 


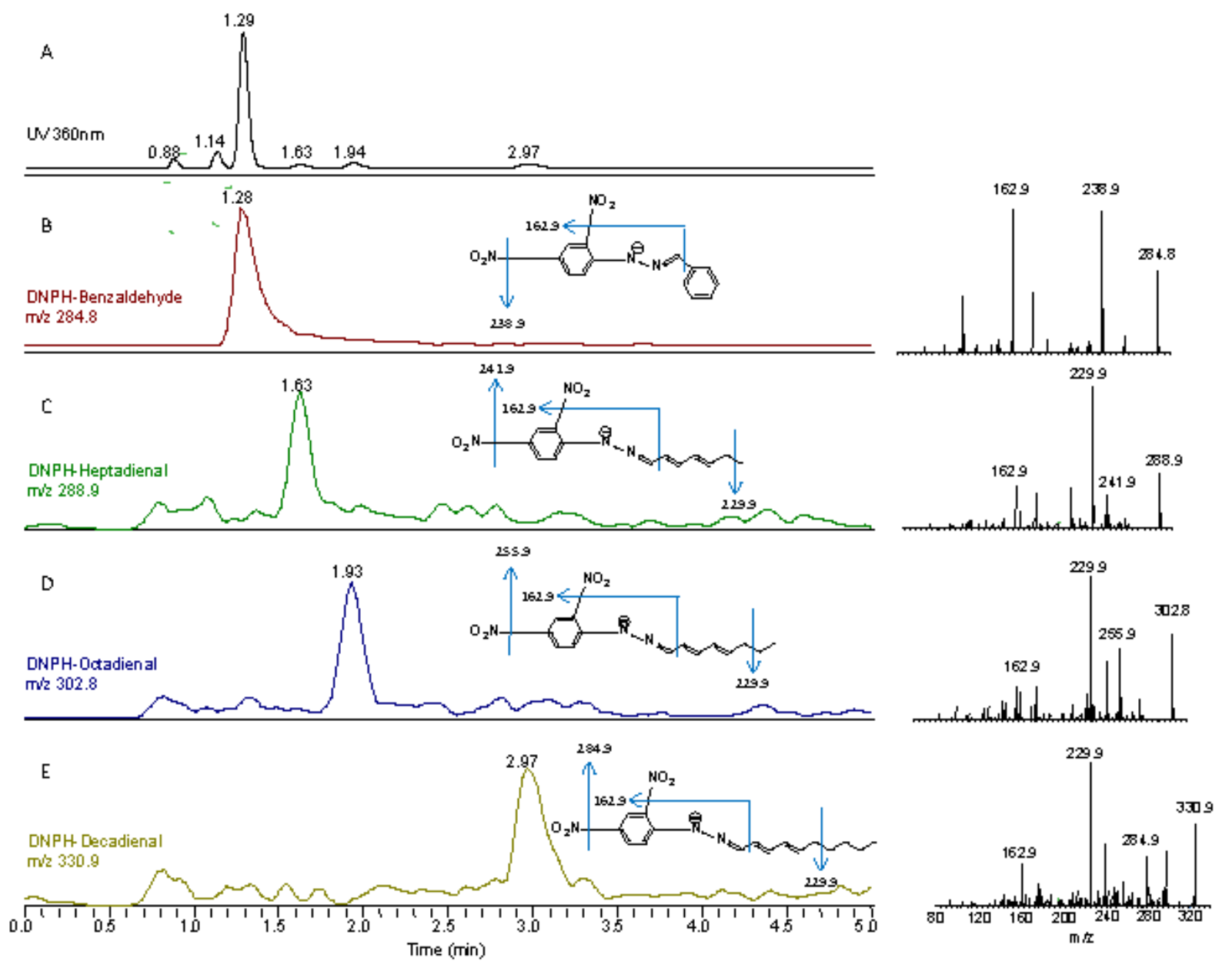

Figure 3. Ion chromatograms and mass spectra from HPLC-APCI-MS analysis of DNPH derivatized PUAs from one control triplicate at $t=$ final ( $24 \mathrm{hr}$ ) from experiment TWNA2. A) An ion chromatogram of UV absorption at $360 \mathrm{~nm}$ shows all DNPH derivatives. B) Extracted ion chromatogram for DNPH-benzaldehyde, structure depicting fragmentation, and $\mathrm{MS}^{2}$ spectrum of $\mathrm{m} / \mathrm{z}=284.8$. C) Extracted ion chromatogram for DNPH-heptadienal, structure depicting fragmentation, and $\mathrm{MS}^{2}$ spectrum of $\mathrm{m} / \mathrm{z}=288.9$. D) Extracted ion chromatogram for DNPH octadienal, structure depicting fragmentation, and $\mathrm{MS}^{2}$ spectrum of $\mathrm{m} / \mathrm{z}=302.8$. E) Extracted ion chromatogram for DNPH-decadienal, structure depicting fragmentation, and $\mathrm{MS}^{2}$ spectrum of $\mathrm{m} / \mathrm{z}=330.9$. 


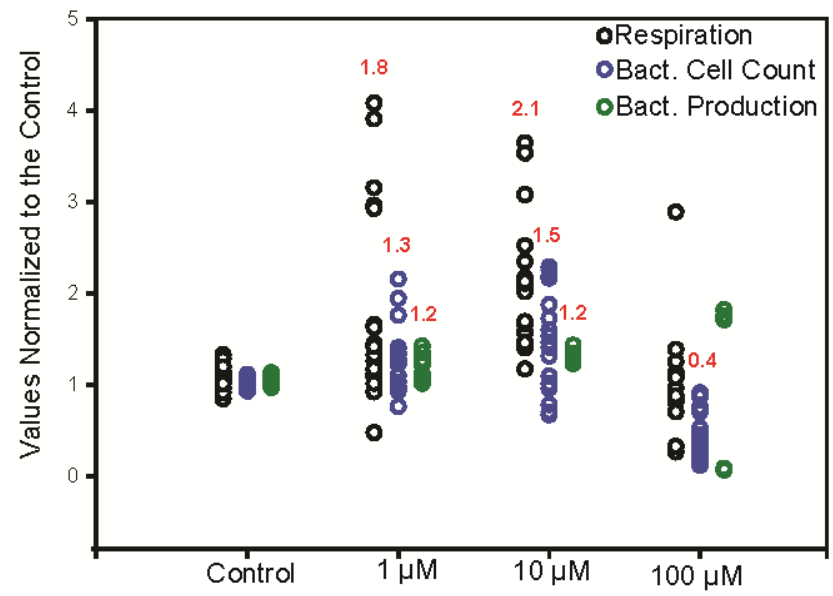

Figure 4. Average effects of PUA treatments on respiration $(n=18)$, production $(n=9)$, and abundance $(n=17)$ of particle-associated cells, presented as the normalized ratio to the noamendment control incubations. Data are derived from triplicate incubations at six stations. In order to isolate and compare the effects of the amendments across stations, results from the incubations at each station were normalized by dividing by the average value of the noamendment control. With the between-station variability removed, differences between treatments and the control were identified using Wilcoxon statistical tests. The red values above the data report the average values for each treatment across all experiments and denote statistical difference from the control, $\mathrm{p}<0.05$ (Wilcoxon Rank Sum). 


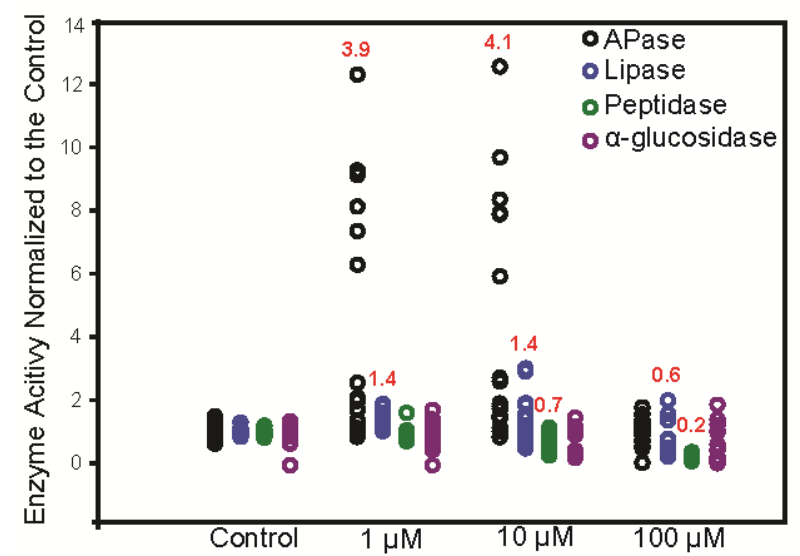

Figure 5. Average effects of PUA amendments on alkaline phosphatase, lipase, aminopeptidase, and alpha-glucosidase activity ( $\mathrm{n}=18$ for all), presented as the normalized ratio to the noamendment control incubations. Statistical analyses conducted as described for Figure 1. The red values above the data report the average values for each treatment across all experiments and denote statistical difference from the control, $\mathrm{p}<0.05$ (Wilcoxon Rank Sum). 
Figure 6. The ion chromatogram and mass spectra from HPLC-APCI-MS analysis of DNPHdecadienal extracted from the $\mathrm{t}=$ initial control treatment in incubation TWNA2. A)

Chromatogram of UV absorption at $360 \mathrm{~nm}$. The internal standard peaks are at 0.89 and 1.34 minutes. The decadienal peak at 3.29 minutes has been amplified by 10x. B) The averaged m/z spectra for retention times 3.21 to 3.41 minutes. The $330.9 \mathrm{~m} / \mathrm{z}$ ion is DNPH-decadienal. The $284.8 \mathrm{~m} / \mathrm{z}$ ion fragment is the result of DNPH-decadienal losing a NO2 group. 


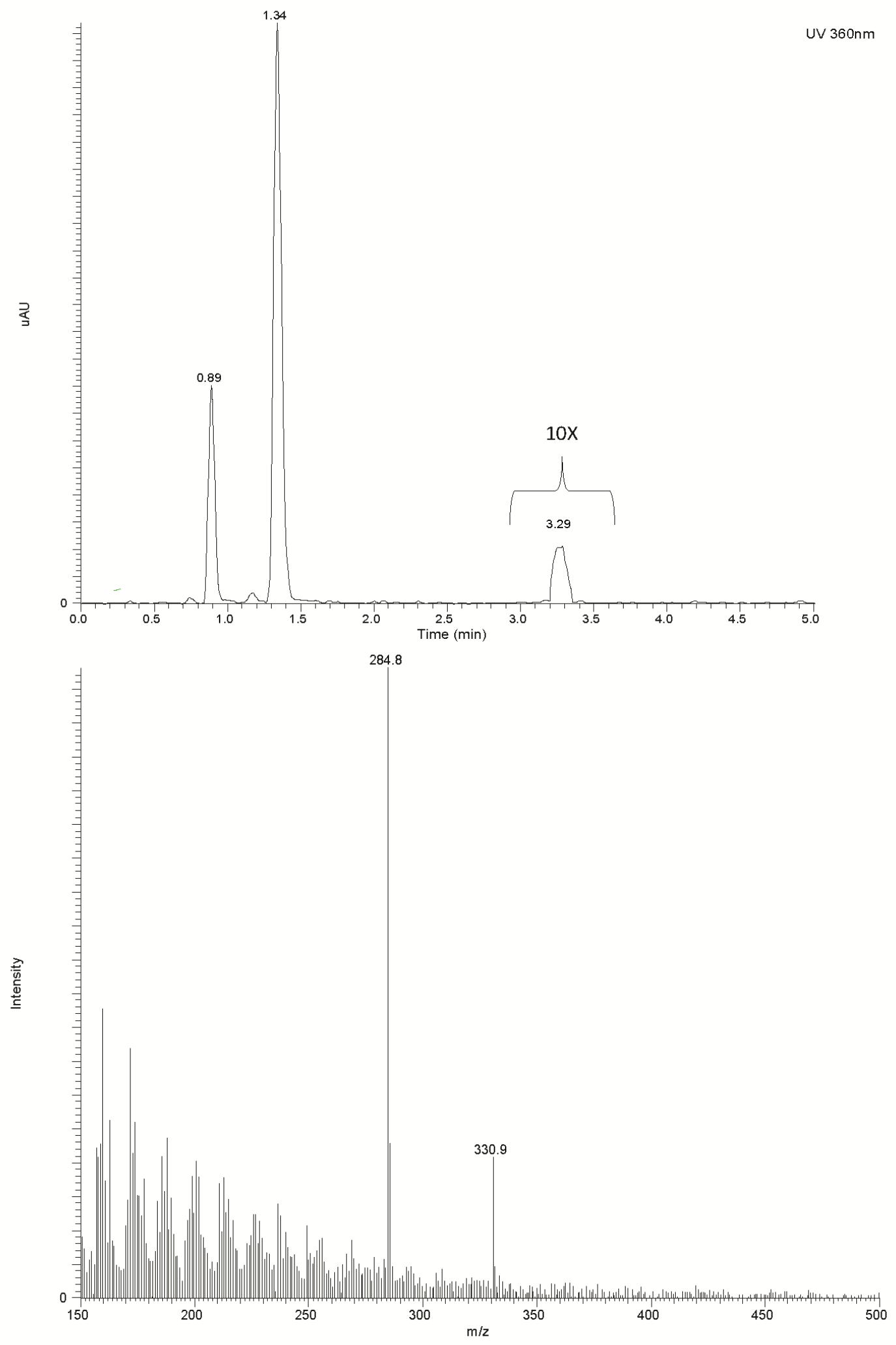




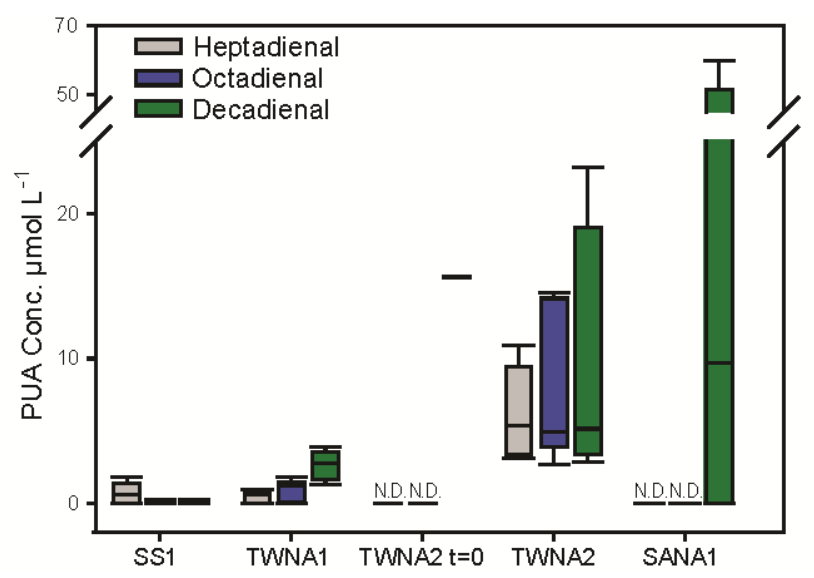

Figure 7. Box plots displaying the average concentration, range of concentrations, and standard deviation from the mean of heptadienal (gray), octadienal (blue), and decadienal (green) ( $\mu$ mol $\mathrm{L}^{-1}$ Particle) for the $\mathrm{t}=$ final control treatments from incubation experiments at SS1, TWNA1, TWNA2, and SANA1 and the $\mathrm{t}=$ initial from TWNA2 ( $\mathrm{n}=3$ for all). These values were estimated from the PUA concentration within the incubations, the POC content of the trap material, and the relationship between POC and volume of diatom-derived marine sinking particulate matter published by Brzezinski et al. (1997).

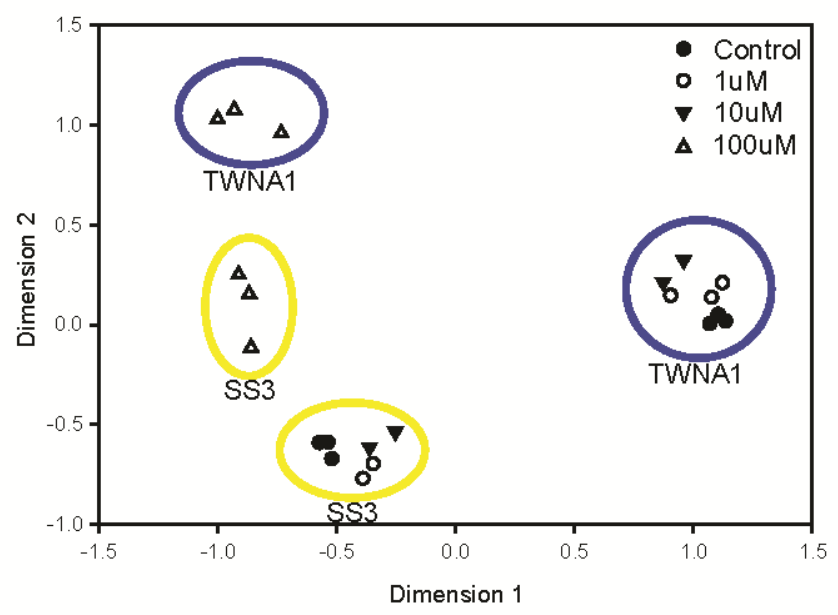

Figure 8. Multidimensional scaling analysis of community structures determined using presence/absence of OTUs analyzed by ARISA. Data points within blue and yellow circles are from experiments TWNA1 and SS3, respectively. Solid black circles are control treatments $(n=6)$. Open circles are $1 \mu \mathrm{M}$ PUA treatments $(\mathrm{n}=5)$. Solid black triangles are $10 \mu \mathrm{M}$ treatments $(n=4)$. Open triangles are $100 \mu \mathrm{M}$ treatments $(n=6)$. 

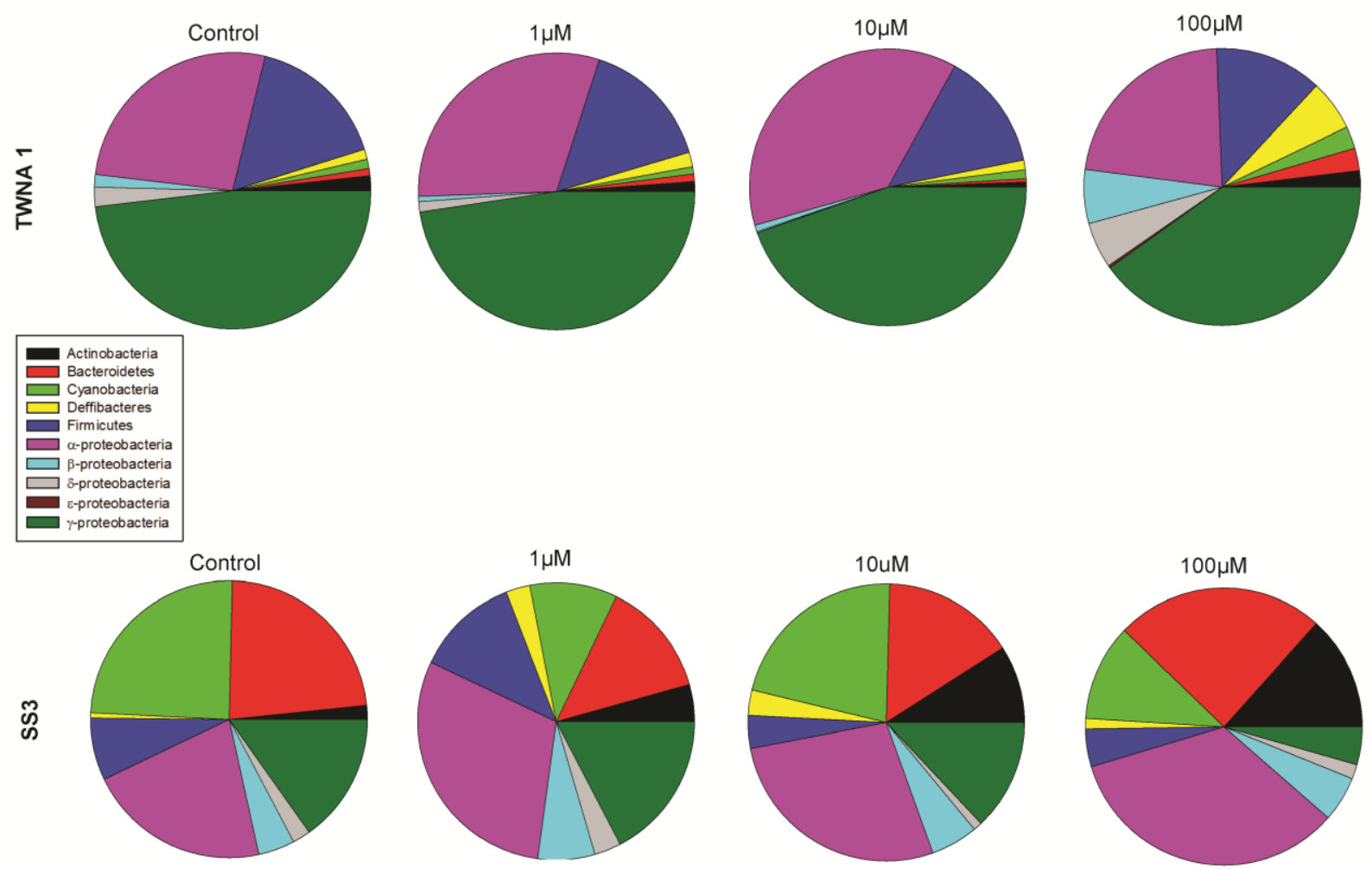

Figure 9. Bacterial community composition within experiments TWNA1 and SS3. For each of the four treatments the pie charts depict the average relative abundance of bacteria within each clade determined by peak area of electropherograms generated using the ARISA technique. Gamma-proteobacteria, Alpha-proteobacteria, and taxa belonging to the Phyla Firmicutes dominated the initial bacterial community from TWNA1, whereas, bacteria falling in to the clades Delta-proteobacteria, Actinobacteria, Beta-proteobacteria, Deferribacteres, Cyanobacteria, and Bacteroidetes were relatively less abundant. The distribution of bacteria amongst the clades shows only minor shifts with the addition of 1 and $10 \mu \mathrm{M}$ PUA. In the $100 \mu \mathrm{M}$ treatment the Alpha- and Gamma-proteobacteria still dominant the community but to a lesser degree compared to the other treatments. The initial bacterial community in experiment SS3 is dominated by Cyanobacteria, Bacteroidetes, Alpha-proteobacteria, and Gamma-proteobacteria. Upon addition of PUAs, Actinobacteria emerges as a dominant clade and Alpha-proteobacteria becomes the most well represented bacterial family, whereas, Gamma-proteobacteria and Cyanobacteria became less abundant. 
Figure 10. Conceptualization of the impact of polyunsaturated aldehydes (PUAs) on seasonal organic carbon export from diatom blooms and subsequent remineralization of organic matter by particle-associated bacteria. Stimulatory concentrations of PUAs lead to enhanced regeneration of nutrients above the winter mixing depth. This allows more nutrients to be entrained into the surface ocean to support a larger diatom blooms during the following year. A possible evolutionary feedback includes enhanced diatom production and concomitant bacteria production. PUA-stimulated bacterial growth and particle disaggregation could also contribute to greater release and retention of PUA-responsive bacteria at shallower depths. 

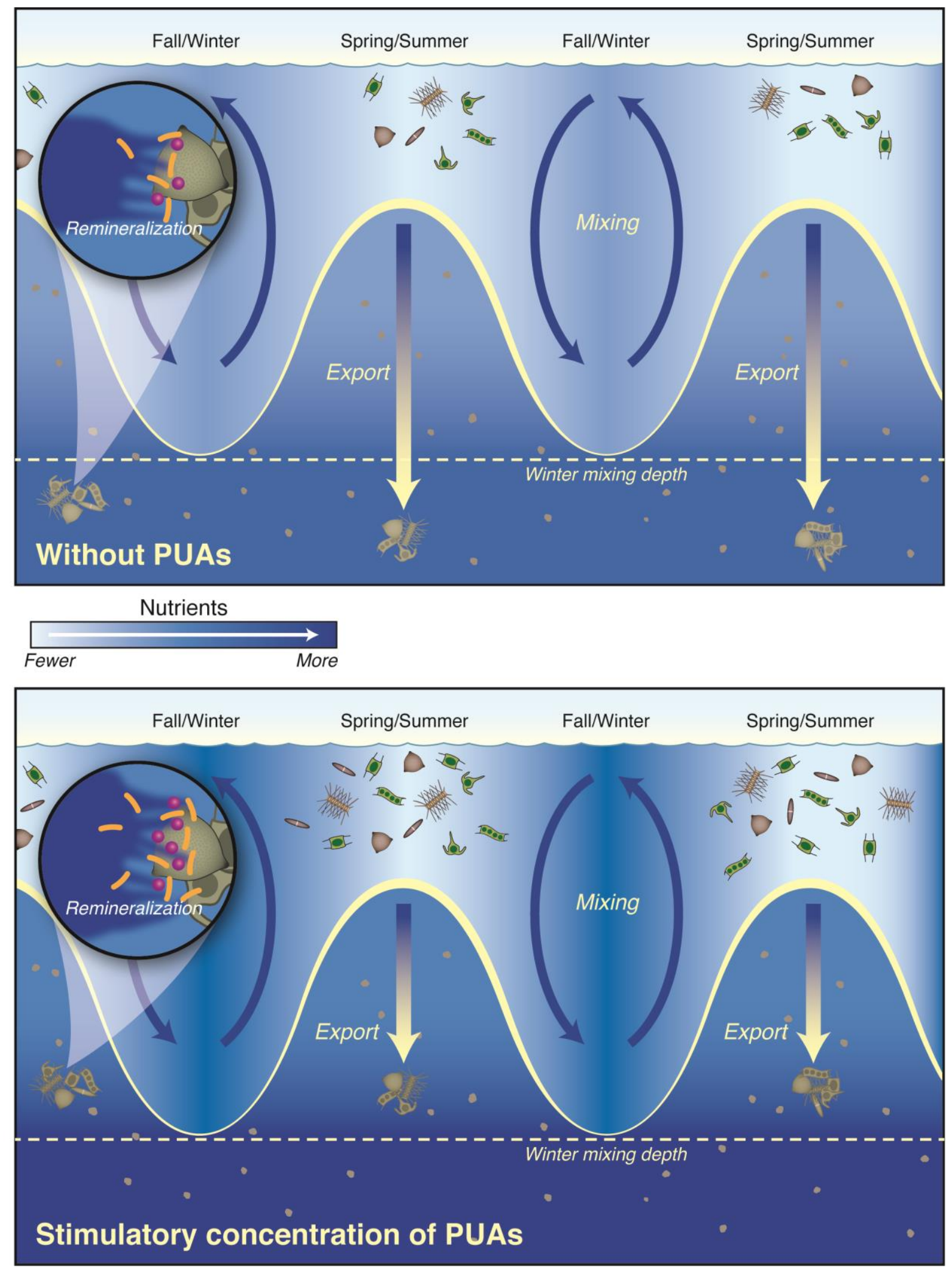
Table S1. Incubation experiment details

\begin{tabular}{|c|c|c|c|c|}
\hline $\begin{array}{l}\text { Experiment } \\
\text { ID }\end{array}$ & Cruise & Trap Location & $\begin{array}{l}\text { POC Content } \\
\mu \mathrm{mol} \mathrm{C} \mathrm{L}^{-1}\end{array}$ & Measurements \\
\hline SS1 & AEx1103 & $\begin{array}{c}33.163 \mathrm{~N} \times 64.335 \mathrm{~W} \\
\text { March 17, } 2011\end{array}$ & 123.4 & $\begin{array}{l}\text { Respiration, Enzyme Activity, Bact. } \\
\text { Cell Abundance, \& PUA Conc. } \mathrm{t}=\text { final }\end{array}$ \\
\hline $\mathrm{SS} 2$ & AEx 1103 & $\begin{array}{c}33.069 \mathrm{~N} \text { x } 64.149 \mathrm{~W} \\
\text { March 19, } 2011\end{array}$ & --- & $\begin{array}{l}\text { Respiration, Enzyme Activity, Bact. } \\
\text { Cell Abundance, \& PUA Conc. } t=\text { final }\end{array}$ \\
\hline $\mathrm{SS} 3$ & KN207-1 & $\begin{array}{c}32.923 \mathrm{~N} \text { x } 65.882 \mathrm{~W} \\
\text { May } 1,2012\end{array}$ & 115.5 & $\begin{array}{l}\text { Respiration, Enzyme Activity, Bact. } \\
\text { Cell Abundance, Bacterial Prod, } \\
\text { ARISA, POC, Pigments, \& PUA } \\
\text { Conc. t=0, final }\end{array}$ \\
\hline TWNA1 & KN207-1 & $\begin{array}{c}38.882 \mathrm{~N} \times 69.035 \mathrm{~W} \\
\text { April 25, } 2012\end{array}$ & 256.1 & $\begin{array}{l}\text { Respiration, Enzyme Activity, Bact. } \\
\text { Cell Abundance, Bacterial Prod, } \\
\text { ARISA, POC, Pigments, \& PUA } \\
\text { Conc. t=0, final }\end{array}$ \\
\hline TWNA2 & KN207-1 & $\begin{array}{c}38.735 \mathrm{~N} \times 69.218 \mathrm{~W} \\
\text { April 27, } 2012\end{array}$ & 117.5 & $\begin{array}{l}\text { Respiration, Enzyme Activity, Bact. } \\
\text { Cell Abundance, Bacterial Prod., } \\
\text { POC, Pigments, \& PUA Conc. } \mathrm{t}=0 \text {, } \\
\text { final }\end{array}$ \\
\hline SANA1 & KN207-3 & $\begin{array}{c}61.787 \mathrm{~N} \text { x } 33.922 \mathrm{~W} \\
\text { July } 10,2012\end{array}$ & 102.8 & $\begin{array}{l}\text { Respiration, Enzyme Activity, Bact. } \\
\text { Cell Abundance, POC, Pigments, \& } \\
\text { PUA Conc. } t=0 \text {, final }\end{array}$ \\
\hline
\end{tabular}

*Links to cruise information can be found on the Biological and Chemical Oceanography Data Management Office (BCO-DMO) website

AEx1103: http://www.bco-dmo.org/deployment/58751

KN207-1:http://www.bco-dmo.org/project/2189

KN207-3:http://www.bco-dmo.org/project/2136 
Table 2. Estimation of the concentration of PUAs in sinking POC

$$
\frac{\text { mol PUA }}{L_{\text {incubation }}} \div \frac{\text { mol POC }}{L_{\text {incubation }}} \times \frac{\text { mol POC }^{\mathrm{a}}}{L_{\text {particle }}}=\frac{\text { mol PUA }}{L_{\text {particle }}}
$$

Concentration in incubation

Concentration in sinking POC (estimated)

\begin{tabular}{|c|c|c|c|c|c|c|c|c|c|}
\hline & {$[\mathrm{Hept}]$} & [Octa] & [Deca] & {$[\mathrm{POC}]$} & $\%$ PUA C & $\begin{array}{c}\text { Particle } \\
\text { volume } \\
\text { (estimated }^{\mathrm{a}} \text { ) }\end{array}$ & [Hept] & [Octa] & {$[$ Deca $]$} \\
\hline & nmol & nmol & nmol & $\mu \mathrm{mol} \mathrm{C}$ & mol PUA C & mmol C & $\mu \mathrm{mol}$ & $\mu \mathrm{mol}$ & $\mu \mathrm{mol}$ \\
\hline & $\overline{L_{\text {incubati }}}$ & $\overline{L_{\text {incubatis }}}$ & $\overline{L_{\text {incubatis }}}$ & $\overline{L_{\text {incubati }}}$ & mol POC & $\overline{L_{\text {particle }}}$ & $\overline{L_{\text {particle }}}$ & $\overline{L_{\text {particle }}}$ & $L_{\text {particle }}$ \\
\hline $\begin{array}{l}\text { SS1 Control } \\
\text { TWNA } 1\end{array}$ & 91.4 & 12.8 & 4.8 & 123.5 & 0.6 & 6.1 & 4.5 & 0.6 & 0.2 \\
\hline Control & 58.0 & 22.2 & 300.7 & 259.4 & 1.4 & 3.8 & 0.9 & 0.3 & 4.4 \\
\hline $\begin{array}{c}\text { TWNA } 2 \mathrm{t}=0 \\
\text { TWNA } 2\end{array}$ & ND & ND & 55.1 & 31.1 & 1.8 & 14.5 & ND & ND & 25.7 \\
\hline $\begin{array}{l}\text { Control } \\
\text { SANA } 1\end{array}$ & 194.3 & 241.0 & 302.0 & 117.7 & 5.4 & 6.3 & 10.4 & 12.9 & 16.1 \\
\hline Control & ND & ND & 514.3 & 103.1 & 5.0 & 6.8 & ND & ND & 34.0 \\
\hline
\end{tabular}

\footnotetext{
${ }^{a}$ Estimated from empirically derived equation for the density of diatom derived organic matter, Volume
} $\left(\mathrm{mm}^{3}\right)=1.628\left[\mu \mathrm{g} \text { POC particle }{ }^{-1}\right]^{1.648}$ (Brzezinski et al., 1997) 
Table 3. Spearman ranked correlation coefficients for comparisons between the relative abundance of each bacterial clade and the concentration of PUAs added for each sample from experiments SS3 (N=10) and TWNA1 (N=11) P<0.01 are statistically significant.

\begin{tabular}{|c|c|c|c|c|c|c|c|c|c|c|}
\hline & 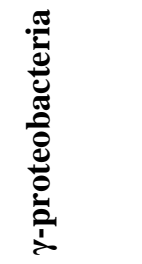 & 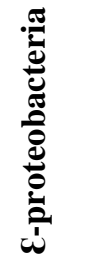 & 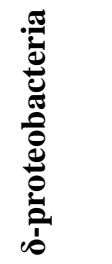 & 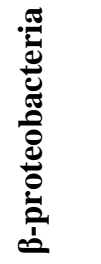 & 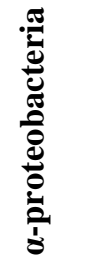 & 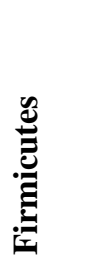 & 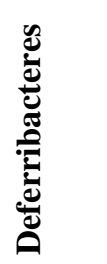 & 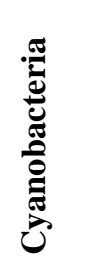 & 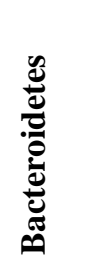 & 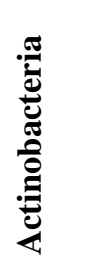 \\
\hline [PUA] & SS3 & & & & & & & & & \\
\hline$r^{2}$ & -0.825 & -- & -0.688 & 0.594 & 0.750 & -0.750 & -0.044 & -0.700 & 0.200 & 0.969 \\
\hline $\begin{array}{l}\mathrm{P} \\
\text { Value }\end{array}$ & 0.001 & -- & 0.025 & 0.060 & 0.011 & 0.011 & 0.892 & 0.022 & 0.559 & 0.000 \\
\hline [PUA] & TWNA1 & & & & & & & & & \\
\hline$r^{2}$ & -0.544 & 0.612 & 0.192 & 0.384 & -0.515 & -0.295 & 0.675 & 0.530 & 0.431 & $\begin{array}{l}- \\
0.145\end{array}$ \\
\hline $\begin{array}{l}\mathrm{P} \\
\text { Value }\end{array}$ & 0.076 & 0.043 & 0.557 & 0.233 & 0.860 & 0.369 & 0.021 & 0.088 & 0.178 & 0.653 \\
\hline
\end{tabular}


Table 4. Comparison of the average change in $\mathrm{O}_{2}$ observed in each incubation and the estimated $\mathrm{O}_{2}$ drawdown necessary to account for the observed removal of PUAs $\left([\mathrm{PUA}]_{\mathrm{t}=\mathrm{initial}}-[\mathrm{PUA}]_{\mathrm{t}=\mathrm{final}}\right)$ via respiration. $\mathrm{P}=\mathrm{Production}$ of PUAs; R =Respiration; D = Drawdown of PUAs; N.D. = Not determined; N.A. = Not applicable

\begin{tabular}{|c|c|c|c|c|c|}
\hline Station & Treatment & $\begin{array}{l}\text { Average } \Delta\left[\mathrm{O}_{2}\right] \\
\mu \mathrm{mol} / \mathrm{L}\end{array}$ & $\begin{array}{l}\text { Average } \Delta[\mathrm{PUA}] \\
\mu \mathrm{mol} / \mathrm{L}\end{array}$ & $\begin{array}{l}\Delta\left[\mathrm{PUA}_{\mathrm{O} 2 \text { equivalents }}\right] \\
\mu \mathrm{mol} / \mathrm{L}\end{array}$ & Comparison \\
\hline$\overline{S S 1}$ & Control & -40.3 & N.D. & N.D. & \\
\hline SS1 & $1 \mu \mathrm{M}$ & -43.3 & N.D. & N.D. & \\
\hline SS1 & $10 \mu \mathrm{M}$ & -63.3 & N.D. & N.D. & \\
\hline SS1 & $100 \mu \mathrm{M}$ & -35.7 & N.D. & N.D. & \\
\hline SS2 & Control & -41.3 & N.D. & N.D. & \\
\hline $\mathrm{SS} 2$ & $1 \mu \mathrm{M}$ & -48.3 & N.D. & N.D. & \\
\hline $\mathrm{SS} 2$ & $10 \mu \mathrm{M}$ & -66.0 & N.D. & N.D. & \\
\hline $\mathrm{SS} 2$ & $100 \mu \mathrm{M}$ & -39.3 & N.D. & N.D. & \\
\hline TWNA 1 & Control & -81.0 & 0.5 & N.A. & $\mathrm{P}$ \\
\hline TWNA 1 & $1 \mu \mathrm{M}$ & -107.7 & -11.0 & -122.1 & $\mathrm{R}<\mathrm{D}$ \\
\hline TWNA 1 & $10 \mu \mathrm{M}$ & -106.3 & -21.2 & -240.9 & $\mathrm{R}<\mathrm{D}$ \\
\hline TWNA 1 & $100 \mu \mathrm{M}$ & -17.3 & 3.5 & N.A. & $\mathrm{P}$ \\
\hline TWNA 2 & Control & -5.7 & 0.7 & N.A. & $\mathrm{P}$ \\
\hline TWNA 2 & $1 \mu \mathrm{M}$ & -25.3 & -0.1 & -5.8 & $\mathrm{R}>\mathrm{D}$ \\
\hline TWNA 2 & $10 \mu \mathrm{M}$ & -13.7 & -0.8 & -11.1 & $\mathrm{R}>\mathrm{D}$ \\
\hline TWNA 2 & $100 \mu \mathrm{M}$ & -5.7 & 25.4 & N.A. & $\mathrm{P}$ \\
\hline SANA 1 & Control & -5.7 & N.D. & N.D. & \\
\hline SANA 1 & $1 \mu \mathrm{M}$ & -1.0 & N.D. & N.D. & \\
\hline SANA 1 & $10 \mu \mathrm{M}$ & -10.1 & N.D. & N.D. & \\
\hline SANA 1 & $100 \mu \mathrm{M}$ & -6.6 & N.D. & N.D. & \\
\hline SS3 & Control & -26.0 & 0.5 & N.A. & $\mathrm{P}$ \\
\hline SS3 & $1 \mu \mathrm{M}$ & -77.3 & -1.8 & -18.2 & $\mathrm{R}>\mathrm{D}$ \\
\hline $\mathrm{SS} 3$ & $10 \mu \mathrm{M}$ & -88.0 & 1.1 & N.A. & $\mathrm{P}$ \\
\hline SS3 & $100 \mu \mathrm{M}$ & -77.7 & -88.8 & -1099.2 & $\mathrm{R}<\mathrm{D}$ \\
\hline
\end{tabular}




\section{Chapter 3}

Microzooplankton grazing and oxidative stress induce oxylipin production in Phaeodactylum tricornutum 


\section{Introduction}

Infochemical compounds produced by diatoms, namely oxylipins, have garnered a lot of attention in marine systems due to their impact on copepod grazers. In the 1990s scientists discovered that copepods fed diets made up exclusively of diatoms had lower reproductive success (Ban and et al., 1997; Peterson, 1994). By examining diatom exudates with high resolution mass spectrometry, lipid derived molecules, decatrienal and decadienal, were determined to be the cause of decreased copepod egg viability (Miralto et al., 1999). These particular compounds are polyunsaturated aldehydes (PUAs) one of the many types of infochemicals produced by diverse oxylipin biosynthesis pathways (Fig 1). The numerous culture studies that followed revealed that PUA production increased when diatoms were grazed or experienced nutrient stress (Pohnert, 2000; Ribalet et al., 2007b). Furthermore, the production of PUAs by diatoms does not appear to deter copepod grazing (Ianora et al., 2004). So PUAs are thought to work as a passive defense mechanism against copepods that allows diatoms to decrease their predators' population size on annual timescales. Over the next 15 years many laboratory, mesocosm, and field studies revealed that oxylipins may also impact bloom dynamics such as phytoplankton community growth (Ribalet et al., 2007a; Ribalet et al., 2008; Vardi et al., 2006), nutrient remineralization (Edwards et al., 2015), biofilm formation (Leflaive and TenHage, 2011), and the fate of carbon exported during bloom termination (Edwards et al., 2015).

Microzooplankton, not copepods, have recently been appreciated as the primary cause of phytoplankton mortality in the surface ocean, consuming 59-75\% of daily phytoplankton growth (Calbet, 2004). Studies that have focused on the impacts of diatom derived infochemicals on the protistan grazers show that unlike copepods, the growth rates of natural microzooplankton populations in the Chesapeake bay were negatively impacted by the addition of exogenous PUAs (Lavrentyev et al., 2015) and compounds released from large volume filtration of mesocosm experiments with PUA producing Skeletonema marioni decreased microzooplankton grazing rates (Stoecker, 2015). Thus, oxylipins produced in situ may act as a chemical defense mechanism towards protistan grazers, potentially decreasing grazing pressure and prolonging blooms.

PUA production in the cosmopolitan diatoms, Thalassiosira rotula and S. marioni has been very well studied making them model organisms for oxylipin research. The biosynthetic 
pathways for PUA production were elucidated by isotopic labeling of potential precursors in these model diatoms (d'Ippolito et al., 2005; d'Ippolito et al., 2006; Fontana et al., 2007a). Like oxylipin production in higher plants, PUA biosynthesis relies on a cascade of enzymatic reactions. First, fatty acids are cleaved from glycolipids and phospholipids that make up the cell membrane (Andreou et al., 2009). Depending on the specificity of the lipoxygenase enzyme (LOX), a hydroperoxy group is added to an alkene carbon moving the position of the double bond over one position. From here the pathway branches, hydroperoxy acids can be transformed into hydroxy epoxy acids by allene oxide synthase (AOS), reduction to hydroxy acids by peroxidases, enzymatically cleaved to PUAs by hydroperoxy lyase (HPL), or non-enzymatically isomerized to keto-acids, leading to a diverse array of oxylipin compounds (Figure 1). The five known oxylipin pathways in diatoms are based on the oxygenation of specific free fatty acids: hexadecactrienoic acid (HDTA; 16:3), hexadecatetranoic acid (HDTrA; 16:4), arachiodonic acid (AA; 20:4), eicosapentaenoic acid (EPA; 20:5), and docosahexaenoic acid (DHA; 22:6) (Fig1). Several of these compounds are known infochemicals, evidence of the ecological and biogeochemical importance of oxylipins (Andreou et al., 2009). Furthermore, the DHA oxylipin pathway was only recently described in diatoms attesting to the prospect of novel compound discovery (Nanjappa et al., 2014).

In order to assess all of the compounds involved in oxylipin biosynthesis, past studies analyzed each class of oxylipin separately by a series of targeted extractions, derivatization steps, and quantification methods. In the case of PUAs, cells were often filtered on to a $0.7 \mu \mathrm{m}$ glass fiber filter $(\mathrm{GF} / \mathrm{F})$ and put through multiple rounds of sonication and/or freeze-thaw cycles to elicit PUA production before extracting the sample, derivatizing aldehyde and ketone groups, and quantifying with GC/MS (Wichard et al., 2005). Therefore, many PUA concentrations reported in the literature represent a potential PUA concentration and do not reflect the dissolved concentration that marine organisms were exposed to in seawater. To quantify the precursor molecules to PUAs, a second filtered sample would be extracted in boiling methanol, separated by liquid chromatography and analyzed with NMR to determine intact lipids and then saponified before analysis with LC-MS/MS to determine the fatty acid composition (d'Ippolito et al., 2004). Thus previous studies do not measure the diversity of oxylipins in a comprehensive, streamlined manner. 
The diversity of oxylipins compounds produced by phytoplankton, the controls on this production, and the bioactivity of the in vivo oxylipidome must be understood in order to place these complex infochemical signaling pathways in an ecological and biogeochemical context. Therefore this study set out to determine which oxylipin compounds were produced by Phaeodactylum tricornutum, how stress impacted the oxylipidome (a subset of the lipidome containing only free fatty acids and oxylipin derivatives), and if there was a link between microzooplankton grazing rates and oxylipin production by their prey.

The diatom Phaeodactylum tricornutum $(\mathrm{Pt})$ was used during these experiments because it is a model organisms that can be genetic modified and one particular transgenic strain, PtNOA, over expresses nitric oxide (NO). No is a robust internal and external signal of stress known to be upregulated in Pt by exposure to decadienal (Vardi et al., 2006). Grazing experiments compared the instantaneous grazing rates of the microzooplankton grazer, Oxyrrhis marina $(\mathrm{Om})$, fed healthy wild-type cells (WT) and chronically stressed cells (PtNOA). The impact of exogenous NO and C20:4 derived oxylipins on microzooplankton grazing rates were also explored, as was the impact of grazing and exogenous oxylipins on NO production by WT. An untargeted lipidomic approach was used to determine the suit of oxylipins produced by healthy WT cells and how the Pt oxylipidome shifts in response to grazing, overproduction of NO, and a combination of the two stress factors. The untargeted lipidomics approach used was suited to the structural diversity of oxylipins produced by diatoms and allowed for relative quantification of free fatty acids, aldehydes, hydroperoxy acids, hydroxy acids, di-hydroxyacids, and keto-acids on one analytical run.

\section{Materials and Methods}

\subsection{Cell culturing}

An axenic culture of the diatom $P$. tricornutum (CCMP2561) was obtained from the National Center for Marine Algae and Microbiota (NCMA), USA, and is referred to as the "wild-type" (WT). A transgenic clone of CCMP2561 overexpressing the ptnoa gene 
was developed by Vardi et al. (2008) and is referred to as PtNOA. A transgenic control, CCMP2561 overexpressing the green fluorescent protein (GFP) was used in some short-term grazing experiments and is referred to as PtGFP. The heterotrophic dinoflagellate Oxyrrhis marina (LB1974) was obtained from The Culture Collection of Algae at the University of Texas, USA, and was fed only WT cells for several months prior to the experiments presented here. Oxyrrhis marina will be referred to as Om.

\subsection{Chemicals}

A NO donor, NOC-5 (3-(2-Hydroxy-1-(1-methylethyl)-2-nitrosohydrazino)-1propanamine), and an extracellular stain for NO, DAFFM (4-Amino-5-Methylamino-2',7'Difluorofluorescen Diacetate) were purchased from Life Technologies, USA. The PUA, 2E,4Zdecadienal (DD) and the NO scavenger Carboxy-PTIO potassium salt were obtained from Sigma-Aldrich, USA. The oxylipins leukotriene B4 (5S,12Rdihydroxy-6Z,8E,10E,14Zeicosatetraenoic acid) and 15(S)-HpETE (15S-hydroperoxy-5Z,8Z,11Z,13E-eicosatetraenoic acid) were purchased from Cayman Chemical (Ann Arbor, MI) for exogenous oxylipin amendment experiments. Benzaldehyde was purchased from Sigma-Aldrich (St. Louis, MO) and used as an internal standard for solid phase extraction of the dissolved lipidome. Synthetic dipalmitoyl- dinitrophenyl-phosphatidylethanolamine (DNP-PE) was purchased from Avanti Polar Lipids, Inc. (Alabaster, AL) and used as the internal standard for extraction of the particulate lipidome. Butylated hydroxytoluene (BHT) was purchased from Sigma-Aldrich and added to all glassware before extraction to prevent oxidation of volatile compounds and adhesion of lipids to the glass. The authentic oxylipin standards: arachidonic acid, docosahexaenoic acid, octanoic acid, 15(S)-HpETE (15S-hydroperoxy-5Z,8Z,11Z,13E-eicosatetraenoic acid), 14,15DiHETE (14,15-dihydroxy-6Z,8E,10E,14Z-eicosatetraenoic acid), 15-HETE (15-hydroxy eicosatetraenoic acid), 14(15)-EpETE (14(15)-epoxy eicosatetraenoic acid), a mix of 5-,12-, and 15-HpETE, 15-HEPE (15-hydroxy eicosapentaenoic acid), 15-HETrE (15-hydroxy eicosatrienoic acid), 9-hydroperoxy octadecadienoic acid (9-HpODE), 13-hydroperoxy octadecadienoic acid (13-HpODE), 9-hydroxy octadecatrienoic acid (9-HOTrE), 13-hydroxy octadecatrienoic acid (13-HOTrE), 9-hydroperoxy octadecatrienoic acid (9-HpOTrE), 13hydroperoxy octadecatrienoic acid (13-HpOTrE), and 9-keto octadecatrienoic acid (9-KOTrE) were purchased from Cayman Chemical (Ann Arbor, MI). The authentic standards 2E,4Z- 
decadienal, 2E,4Z-octadienal, and 2E,4Z-heptadienal were purchased from Sigma-Aldrich. These authentic standards were also used to verify putative annotations in the lipidome.

\subsection{Laboratory grazing experiments, cell enumeration, and physiology}

Grazing experiments were conducted by Matt Johnson (Johnson et al., submitted). Experiments were set up in both $50 \mathrm{ml}$ culture flasks and 24 well tissue culture plates, at $18^{\circ} \mathrm{C}$ and in $50 \mu \mathrm{mol}$ photons $\mathrm{m}^{-2} \mathrm{~s}^{-1}$. Six types of grazing experiments were conducted measuring:
a) 10 minute instantaneous ingestion rates of WT, PtNOA, WT + NO donor (Figure 4A)
b) 24 hour ingestion rate of WT and PtNOA as a function of predator : prey (Figure 4B)
c) 24 hour ingestion rate of WT as a function of NO concentration (Figure 4C)
d) 24 hour ingestion rate of WT and PtNOA exposed to varying concentrations of decadienal (Figure 4D)
e) 24 hour ingestion rate of WT and WT + NO-Scavenger exposed to varying concentrations of decadienal (Figure 4E)
f) $48 \mathrm{hr}$ ingestion rate of WT exposed to varying concentrations of the NVOs, 15(s)- HpETE and Leukotriene B4 (Figure 4F)

For density dependent grazing experiments (Figure 4B), O. marina and P. tricornutum were combined at various concentrations in sterile filtered seawater, and growth and grazing rates were determined. Otherwise, the predator to prey ratio was 1:9. Grazing experiments were conducted in the presence of a variety of chemicals, including decadienal (between 0.1-5 $\mu \mathrm{M}$ ), 15(S)-HpETE and Leukotriene B4 (between 0.5-500 nM), a NO donor (NOC-5: 20-500 $\mu \mathrm{M}$ ), a NO stain (DAF-FM: $50 \mu \mathrm{M}$ ), and a NO scavenger (CPTIO: $200 \mu \mathrm{M}$ ).

For instantaneous $(<1 \mathrm{~h})$ ingestion rates (IR'), ingested $P$. tricornutum $(\mathrm{Pt})$ within $O$. marina $(\mathrm{Om})$ food vacuoles were enumerated using fluorescence microscopy (Ex BP 450490nm, Em LP 515nm) on a Zeiss Axio scope A1, at 0, 10, 20, 30, and 60 min (Li et al., 2001). Cells were preserved with $1 \%$ gluteraldehyde and filtered onto black polycarbonate $2 \mu \mathrm{m}$ nucleopore filters, and at least 100 cells were enumerated for ingested prey. For longer experiments (24-48h), changes in Pt cell abundance with and without Om were determined using a Guava (Millipore) flow cytometer at 0,24, and 48h. Growth and grazing rates were calculated 
using the equations of Frost (Frost, 1972) and Heinbokel (Heinbokel, 1978) ( Table 1, Figure 4A-F).

Fluorescence of DAF-FM, a NO stain, was measured using a Millipore Guava easyCyte flow cytometer at $488 \mathrm{~nm}$ in an experiment that measured the production of NO by WT under four conditions in triplicate: prey only control, WT + Om, and WT amended with 0.2 and $5 \mathrm{nM}$ decadienal (Figure 5).

\subsubsection{Statistical analysis of the grazing experiments and physiological measurements.}

Analysis of variance (ANOVA) with HSD was used to determine differences in ingestion rates of $\mathrm{Pt}$ by $\mathrm{Om}$ in all grazing experiments, growth rates of $\mathrm{Pt}$ and $\mathrm{Om}$ in amendment experiments with exogenous oxylipins, and NO production by WT under grazing pressure and decadienal exposure ( $\mathrm{p}$-value $<0.05)$.

\subsection{Preparation of the Lipidome}

Samples were collected for complete lipidome analysis from four treatments, WT, PtNOA, WT + Om, and PtNOA + Om, $6 \mathrm{hr}$ after the start of the grazing experiment. Particulate lipid samples were collected by filtering $200 \mathrm{ml}$ of each treatment onto $0.2 \mu \mathrm{m}$ Durapore filters. The filters were stored at $-80^{\circ} \mathrm{C}$ and extracted in the lab using a modified Bligh and Dyer (1959) lipid extraction protocol (Bligh and Dyer, 1959; Popendorf et al., 2013). DNP-PE was added as an internal standard during extraction, as was the antioxidant BHT.

Samples for dissolved lipids were collected by pre-filtering $200 \mathrm{~mL}$ of each treatment through a $0.2 \mu \mathrm{m}$ sterivex filter to remove particulates. Benzaldehyde was added to the filtrate as an internal standard $(10 \mu \mathrm{M})$. Dissolved lipids were then extracted onto a solid phase extraction cartridge (Waters HLB) and stored at $-80^{\circ} \mathrm{C}$ until elution. Once the dissolved lipids were eluted off of the SPE cartridges with methanol, the sample was immediately analyzed to avoid autooxidation of the compounds. BHT, a commercial antioxidant, was also added to the collection vials before elution to prevent degradation of the samples.

Both particulate and dissolved lipidome samples were analyzed in positive and negative mode using reverse phase HPLC (Agilent 1200 system ; Agilent, Santa Clara, CA, USA) paired with high resolution, accurate mass (HRAM) data from a Thermo Exactive Plus Orbitrap mass 
spectrometer (ThermoFisher Scientific, Waltham, MA, USA). This method was adapted from Hummel et al. (2011). A $20 \mu \mathrm{L}$ aliquot of sample was injected onto a C8 Xbridge HPLC column (particle size $5 \mu \mathrm{m}$, length $150 \mathrm{~mm}$, width $2.1 \mathrm{~mm}$; Waters Corp., Milford, MA, USA) carried by Eluent A (water with $1 \% 1 \mathrm{M}$ ammonium acetate and $0.1 \%$ acetic acid) and Eluent $\mathrm{B}(70 \%$ acetonitrile and $30 \%$ isopropanol with $1 \% 1 \mathrm{M}$ ammonium acetate and $0.1 \%$ acetic acid ). The starting gradient was $45 \%$ Eluent A and 55\% Eluent B. Over the 30 minute run, the gradient shifted to $1 \%$ Eluent A and $99 \%$ Eluent B. The first minute of the run was diverted to waste. From 1 to 30 minutes the Orbitrap mass spectrometer collected full scan and all-ionfragmentation data in positive and negative mode for a mass range of $100-1500 \mathrm{~m} / \mathrm{z}$ at a mass resolution of 170,000 . Only the negative mode data was presented here since the molecules of interest, free fatty acids and oxylipins, form negative ions under electrospray ionization. However, the recovery efficiency of the internal standard, benzaldehyde, was extracted from the positive mode data. Additionally, authentic standards of seventeen oxylipins were diluted in methanol to a final concentration of $10 \mu \mathrm{M}$ and analyzed separately using the above method.

\subsection{Annotation of the particulate and dissolved lipidomes}

The particulate and dissolved lipidomes were annotated to the structural level in the software package MAVEN (Metabolic Analysis and Visualization ENgine) which detected peaks and facilitated structural level annotation by querying a boutique lipid database containing the various adducts of intact polar lipids, fatty acids, triacylglycerols, and their oxylipin derivatives (Collins et al., in prep; Melamud et al., 2010). Structural level annotation corresponded to oxylipin subclass (i.e. fatty acid, NVO, or PUA) and designated the number of carbons, double bonds, and oxygenations based on the exact mass. Putative structural level annotations were only assigned to features that were greater than $10^{4}$ intensity units with at least one peak across all samples with a maximum quality score of 0.8. Putative structural level annotations in MAVEN were based solely on $\mathrm{m} / \mathrm{z} \pm 2 \mathrm{ppm}$ for NVOs and $\pm 5 \mathrm{ppm}$ for PUA with a maximum of 3 best matches. Structural level annotations were parsed down to the "best match" assignments based on broad retention time criteria (1-18 mins), the assumption of acetogenic lipid production in $\mathrm{Pt}$ (i.e. only even chain-length lipids), and dominance of the $[\mathrm{M}-\mathrm{H}]^{-}$adduct (Collins et al., in prep). 
Of the 223 features assigned putative structural annotations, 126 were annotated as free fatty acids, PUAs, or non-volatile oxylipins.

A suit of 23 authentic standards was used to further annotate features in the lipidome to the functional group level based on retention times (RT) (Table 2). Functional group level annotation described the class of oxylipin based on the oxygenated functional group present (i.e. hydroxy acids, hydroperoxy acids, and hydroxy epoxy acids). The authentic standards encompassed a diverse range of molecules including free fatty acids, NVOs with 1 or 2 oxygens (-1O and -2O), and PUAs. First the observed RTs ( $\left.\mathrm{RT}_{\mathrm{obs}}\right)$ of features in the lipidome with structural level annotations matching the authentic standards were compared to the RTs of authentic standards $\left(\mathrm{RT}_{\text {auth }}\right)$ (Table 3). For $-2 \mathrm{O}$ NVO, $\mathrm{RT}_{\text {obs }}$ within 0.7 minutes of the $\mathrm{RT}_{\text {auth }}$ of standards were assigned putative functional group level annotations. For -1O NVO, fatty acid, and PUAs $\mathrm{RT}_{\text {obs }}$ within 0.5 minutes of the $\mathrm{RT}_{\text {auth }}$ were assigned putative functional group level annotations. These RT windows were determined by the spread in retention times observed for the positional isomers 5-, 12-, and 15- HpETE and 12- and 15-HETE (Table 2).

Next the $\mathrm{RT}_{\text {obs }}$ of features in the lipidome that were annotated as other fatty acids, $-1 \mathrm{O}$ NVOs, -2O NVOs, and PUAs were compared to RT predicted $\left(\mathrm{RT}_{\text {calc }}\right)$ from the authentic standards. The difference between the retention times of C20:3 and C18:3 structural analogs, 15HETrE (RT-6.1) and 13-HOTrE (RT-4.0), was 2.1 minutes, suggesting that RT increased 1.05 minutes per additional carbon to the alkyl chain(Figure 2A). The differences between retention times of the C20:5 and the C20:4 hydroxy acid standards, 15-HEPE (RT- 4.6) and 15-HETE (5.4), suggested that the addition of a double bond (DB) increased the retention time of HEPE by 0.9 minutes (Figure 2B). Therefore, the predicted RTs were calculated using Equation 1 (Table 4a-g). $\mathrm{RT}_{\text {obs }}$ within 0.7 minutes of the RT predicted by the $-2 \mathrm{O}$ NVO standards and within 0.5 minutes of the RT predicted by the -10 , fatty acid, and PUAs standards were assigned putative functional group level annotations (Table 4a-h).

$$
\begin{gathered}
\text { Eq. } 1 \quad R T_{\text {calc }}=R T_{\text {auth }}-\left(\# \text { carbons }_{\text {auth }}-\# \text { carbons }_{\text {obs }}\right) * \frac{1.05 \mathrm{~min}}{\text { carbon }} \\
+\left(\# D B_{\text {auth }}-\# D B_{\text {obs }}\right) * \frac{0.9 \mathrm{~min}}{D B}
\end{gathered}
$$


Features putatively annotated as PUAs that did not match the RT predicted by the authentic standards were re-annotated at the elemental formulas level (Table $4 \mathrm{~h}$ ). These $\mathrm{m} / \mathrm{z}$ were then queried against the Kyoto Encyclopedia of Genes and Genomes (KEGG) database since PUAs can be isomers of small metabolites (Table 5). NVO that did not match the RT predicted by the authentic standards were not re-annotated because authentic standards were not available for all of the possible NVO and there were fewer overlapping metabolites.

A confidence ranking system ranging from $1-5$ was employed to describe the certainty of these putative annotations (Fig 3). Level 1 denotes the highest level of confidence and requires an elemental formula determined by exact mass, RT match to a standard, and two diagnostic mass spectra. Because all-ion fragmentation, a less selective ionization method, was used there were no Level 1 annotations. Level 2 annotations required an elemental formula determined by exact mass and a RT in agreement with an authentic standard. The Level 2 confidence ranking was further broken down into $2 \mathrm{a}$ and $2 \mathrm{~b}$ annotations. The letter "a" denoted that the $\mathrm{RT}_{\text {obs }}$ was exactly equal to the $\mathrm{RT}_{\text {auth. }}$. The letter "b" denoted that the $\mathrm{RT}_{\mathrm{obs}}$ was within 0.7 min of the $\mathrm{RT}_{\text {auth }}$ for $-2 \mathrm{O}$ NVOs or $0.5 \mathrm{~min}$ of the $\mathrm{RT}_{\text {auth }}$ for $-1 \mathrm{O}$ NVOs, fatty acids, and PUAs. Level 3 annotations required an elemental formula determine by exact mass, a structural level annotation, and a RT in agreement with RT predicted from authentic standards. Both levels 2 and 3 of this confidence ranking system represent proposed functional group level annotations. Level 4 annotations had an elemental formula determined by exact mass and structural level annotation but were not matched to retention times proposed by the suite of authentic standards. The level 4 confidence ranking represented a proposed structural level annotation consistent with oxylipin subclasses (i.e. non-volatile oxylipins, PUAs, and free fatty acids). All structural level annotations without confidence level in parentheses were level 4 annotations. All features annotated as NVOs with 3 or 4 oxygens were level 4 annotations because these types of molecules were not included in the set of standards. Level 5 annotations represented unknown compounds that had an elemental formula determined by exact mass but were excluded from the proposed oxylipin subclass based on RT. The level 5 annotations in this study were compounds originally annotated as PUAs but eluted much earlier than the PUA standards would predict thus the structural level annotation was rejected and the features were assigned an elemental formula level annotation. A search against the KEGG database suggested that these small $(\mathrm{m} / \mathrm{z}<200)$, 
early eluting compounds might be terpenoid alcohols or cyclized metabolites with keto groups, many of which are bioactive (Table 5).

\subsection{Normalization and relative quantification of molecular species}

The peak area of each identified component was normalized to benzaldhyde in the dissolved lipidome and DNP-PE in the particulate lipidome. Level scaling was applied to the data by dividing each peak area, $\mathrm{x}_{\mathrm{j}}$ by the average peak area of that feature across the samples, $\mathrm{x}_{\mathrm{avg}}$ (Equation 2). Level-scaling allowed for relative quantification, observing the change in the abundances of molecular species across treatments. Because an untargeted approach was taken, there was not adequate information to account for variations in extraction efficiency and ionization efficiency across the diversity of molecular species observed. Therefore, quantification was relative not absolute. The level-scaled peak areas of each molecular species were averaged across the triplicates for each treatment $(\mathrm{N}=3)$ (Figure $6 \mathrm{~B})$. This data was used to generate a heatmap depicting the $\log 2$ transformed fold change in peak area of each molecular species relative to the average across the treatments.

$$
\text { Equation (2) } \quad x_{\hat{\jmath}}=\frac{x_{j}}{x_{a v g}}
$$

\subsection{Structural and statistical analysis of the dissolved and particulate lipidomes}

A Euclidean distance matrix of the data was calculated and used to build linkage trees describing the similarities in the lipidomes across treatments and the variations of individual compounds across treatments. Similarity profile analysis was applied to both dendrograms to determine which treatments and clusters of molecules were significantly similar using the simprof function with in the R package sigclus (Figures 4A, 4C, 6A, and 6B). A permutational multivariate analysis of variance (PerMANOVA) technique was also applied to the hierarchical clustering of the treatments to determine if any experimental meta-data (Table 6) could adequately explain the clustering patterns (R vegan package, adonis function) (Table 7).

Lastly, a series of paired t-tests were used to determine which molecular species were significant different between the treatments (Figure 7 and 8B). Nested t-tested were run to test the following six comparisons: a) WT vs PtNOA, b) WT vs WT+Om, c) WT vs PtNOA+Om, d) 
PtNOA vs WT+Om, e) PtNOA vs PtNOA + Om, and f) PtNOA+Om vs WT+Om. A p-value of 0.1 was chosen and the Bonferroni correction was applied to account for Type I errors, lowering the p-value to 0.0167 . Only the molecular species that varied most significantly with a p-value< 0.0167 were presented in Figure 7. All molecular species that varied significantly between treatments with a p-value $<0.1$ were presented in Figure 8 with the degree of significance denoted.

\section{Results}

\subsection{Grazing Experiments}

Instantaneous ingestion rates (IR') of $O$. marina on PtNOA and WT pretreated with 400 $\mu \mathrm{M}$ of the NO donor NOC-5 for 2 hours were significantly lower than ingestion rates of untreated wild-type cells (ANOVA, F3, $4=15.7$, p = 0.0263; Fig. 4A). Grazing of PtNOA was lower relative to WT when grazing was assayed as a function of prey concentration and the predator : prey ratio was greater than or equal to 20 (ANOVA, F11,12=43.16, p = 6.4 x 10 $0^{-8}$; Fig. 4B). Density-dependent differences in grazing by $O$. marina due to NO production by PtNOA resulted in lower saturation of the IR curve. The effect of NO production on grazing also exhibited dose dependence with reduced IR as concentrations of the NO donor, NOC-5, reached levels of 100-500 $\mu \mathrm{M}$ (Figure 4C). Despite observed declines in grazing rates, the growth rate of O. marina $(\mu)$ was not affected over the 24 or $48 \mathrm{~h}$ grazing experiments (Table 1).

Grazing experiments adding a series of exogenous arachidonic acid (C20:4) oxylipins were also conducted. Decadienal, HpETE, and leukotriene B4 (di-hydroxy-ETE) were tested separately over a range of concentration. Any addition of decadienal led to a decrease in grazing rate relative to the no-amendment control (Figure 4D). From 0 to $0.1 \mu \mathrm{M}$ decadienal, the grazing rate of $O$. marina on WT is the same as the grazing rate on PtNOA. However at $0.5 \mu \mathrm{M}$ decadienal, WT and PtNOA grazing rates diverge with $O$. marina grazing WT cells at a rate of $90 \%$ the control and PtNOA cells at a rate of $60 \%$ the control. As the decadienal concentration increased to $1 \mu \mathrm{M}$ the gap between ingestion rates of WT and PtNOA continued to grow. At the highest concentration tested, $5 \mu \mathrm{M}$, grazing of both WT and PtNOA converged at less than a third of the no-amendment control. The effect of decadienal on grazing rates could be 
ameliorated by the addition of CPTIO, a NO scavenger, to decadienal grazing experiments (Fig 4E). At 0.05 and $5 \mu \mathrm{M}$ decadienal, the ingestion rate of WT recovered to control levels when treated with a NO scavenger. The link between NO production and oxylipin exposure was shown by measuring NO produced by WT cells exposed to varying concentrations of decadienal. After 2 hours, NO production by WT exposed to 0.2 and $5 \mu \mathrm{M}$ decadienal was equivalent to NO production by grazed WT (Figure 5).

In contrast to decadienal, exposure to the C20:4 oxylipins resulted in significantly lower grazing at much lower concentrations. The oxylipin 15(s)- HpETE resulted in significant declines in IR (ANOVA: F7,40 = 5.9, p = 5.93 x 10 ${ }^{-5}$ ) that were observed at or above $1 \mathrm{nM}$ (Fig. 4F; black circles). Similar results were also observed for leukotriene B4 with lower IR at or above $0.5 \mathrm{nM}\left(\mathrm{F} 7,40=22.3, \mathrm{p}=6.2 \times 10^{-12}\right.$; Fig.2F; white circles $)$. While stronger responses were observed for C20:4 oxylipins relative to decadienal, the full effects required $48 \mathrm{~h}$ of exposure. Grazing responses to decadienal only required $24 \mathrm{~h}$ and did not increase with time.

No changes were observed in growth rates $(\mu)$ of $O$. marina in any of the decadienal amendment experiments over 24 or $48 \mathrm{~h}$ (Table 1), despite observing significant declines in several grazing parameters (Figure 4D). For C20:4 oxylipin experiments, $O$. marina $\mu$ was lower in only one experiment at 50,100, and $500 \mathrm{nM}$ 15(s)-HpETE (Table 1; ANOVA: $\mathrm{F}_{7,16}=4.82$, p= $4.41 \times 10^{-3}$; HSD: $\mathrm{p}_{50 \mathrm{nM}}=0.013, \mathrm{p}_{100 \mathrm{nM}}=0.006$, and $\left.\mathrm{p}_{500 \mathrm{nM}}=0.006\right)$. In contrast, $\mu$ of $P$. tricornutum was significantly affected by decadienal in all experiments ( Table 1; ANOVA: $\mathrm{F}_{6,113}=12.43, \mathrm{p}=1.02 \times 10-10$; HSD: $\left.\mathrm{p}=1.1 \times 10^{-6}\right)$ and by 15(s)-HpETE at the highest concentrations tested, $500 \mathrm{nM}$ (ANOVA: $\mathrm{F}_{7,40}=3.059, \mathrm{p}=0.011$; HSD: $\mathrm{p}=0.026$ ). No effect was observed for leukotriene B4 on $\mu$ of $P$. tricornutum.

\subsection{Dissolved Free Fatty Acids and Oxylipins}

\subsubsection{Relative Quantification Using Comparative Lipidomics.}

A comparative lipidomics approach was used for the relative quantification of free fatty acids and oxylipin derivatives within the dissolved lipidomes of the four treatments (WT, PtNOA, PtWT+Om, and PtNOA+Om). Average normalized relative quantification revealed 
distinct shifts in the lipidome of $P$. tricornutum in response to stress. Differential regulation patterns were observed amongst the molecular species with both up- and down-regulation across the treatments, underscoring the complex lipidomic response to stress (Figure 6B).

\subsubsection{Similarities in lipidome structure between treatments}

Overall similarities between lipidomes were assessed by hierarchical cluster analysis of the treatments based on their average normalized lipidomes using similarity profile analysis to identify significantly distinct clusters (Figure 6A). The WT lipidome (red) formed a separate branch from the three stressed treatments which formed two significantly distinct clusters (green and blue). The lipidomes of the two grazed treatments, $\mathrm{WT}+\mathrm{Om}$ and PtNOA $+\mathrm{Om}$, were most similar to one another forming a significant cluster. The PtNOA lipidome (green) was more similar to the lipidome of the grazed treatments than to the WT lipidome. Thus, the dissolved lipidome of $P$. tricornutum was significantly different under chronic stress induced by over production of $\mathrm{NO}$ and under acute stress due to microzooplankton grazing. However, grazing had a larger impact on the dissolved lipidome than overproduction of NO. The two grazing treatments were statistically similar and "permutational" multivariate analysis of variance determined that the lipidomic similarities between treatments were most attributable to the presence/absence of the grazer $\left(\right.$ Table $7, \mathrm{R}^{2}=0.19605 \mathrm{p}$-value $=0.033$ ).

\subsubsection{Oxylipin and free fatty acids significantly upregulated in response to stress}

Individual molecular species that varied most significantly between the treatments ( $p$-value $<0.0167)$ were determined by a series of nested t-tests making a total of six comparisons between the treatments and applying a Bonferroni correction. Comparing the lipidomes of WT and WT + Om yielded the largest number of significantly different compounds with fourteen molecular species upregulated and two molecular species downregulated in the grazed treatment relative to the prey only WT treatment (Figure 7). Grazing of PtNOA (PtNOA $+\mathrm{Om}$ ) only resulted in three significantly upregulated molecules relative to WT and no significantly regulated molecules relative to the prey only PtNOA treatment. Two molecular

species were significantly regulated between the two prey only treatments, WT and PtNOA. 
Comparison between the two grazed treatments ( $\mathrm{WT}+\mathrm{Om}$ vs. PtNOA $+\mathrm{Om}$ ) showed no significant difference in the relative abundance of any of the dissolved oxylipins.

\subsubsection{Differential production patterns of free fatty acids and oxylipin compounds in response to} stress

Hierarchical cluster analysis was also applied to the relative abundance of molecular species in order to identify groups of molecules that varied similarly across treatments. Using similarity profile analysis, eighteen significantly distinct groups were identified (Figure 6C). The twenty-two most significantly differing molecular species determined in the previous section were distributed amongst nine groups and are highlighted in Figure 6. Significantly differing molecular species made up nearly half of compounds in Groups 16 and 18. Group 10 was composed of a single significantly varying compound, PUA 9:2 RT-1.48. This analysis suggested that the nested t-tests with a Bonferroni correction may be too rigid of a statistical test leading to Type II errors. When the Bonferroni correction is removed, the number of significantly varying compounds increased from 22 to 58 (Fig 8).

\subsection{Particulate Free Fatty Acids and Oxylipins}

\subsubsection{Untargeted annotation and relative quantification using comparative lipidomics}

The same comparative lipidomics approach described for the dissolved lipidome was used to analyze the particulate lipidome. The particulate lipidome had 500 features in negative ionization mode, of which 19 were annotated as free fatty acids or oxylipins (Figure 9B). The particulate oxylipidome was small relative to the dissolved oxylipidome (19 compounds as opposed to 126; Figure 6B). The only previously described diatom oxylipins observed in the particulate lipidome were the 20:5 and 22:6 free fatty acids which are oxylipin precursors. The particulate lipidome was not annotated to the functional group level. 


\subsubsection{Similarities in lipidome structure between treatments}

Hierarchical and similarity profile analyses showed that the particulate lipidomes of grazed cells were significantly more similar than the particulate lipidomes of prey onlyWT and PtNOA treatments (Figure 9A). Despite the two grazing treatments clustering together, none of the molecular species in the particulate lipidome of the grazed WT treatment (WT+Om) were found to be significantly regulated ( $\mathrm{p}<0.00167$ ), whereas, four molecular species were upregulated in the grazed PtNOA treatment (PtNOA+Om) relative to the two prey only control treatments: DHA (FFA 22:6), docosaenoic acid (FFA 22:1), docosatetraenoic acid (FFA 24:1) and an saturated C14 fatty acid with two additional oxygen (FFA 14:0-2O) (Fig 9B). Two molecular species were found to be significantly more abundant in grazed PtNOA relative to grazed WT, EPA (FFA 20:5) and a dodecadienoic acid with one additional oxygen (FFA 12:2 10).

\section{Discussion}

\subsection{Linking phytoplankton stress to microzooplankton grazing deterrence}

The microzooplankton grazer $O$. marina exhibited faster grazing when fed healthy WT cells than when fed chronically stressed cells (PtNOA), both in terms of instantaneous ingestion rates and 24 hour density dependent ingestion rates (Figure 4A and 4B). The transgenic strain PtNOA overexpresses the gene ptnoa, resulting in over production of nitric oxide (NO) a robust stress signal used in most intracellular signaling cascades across kingdoms (Beligni and Lamattina, 2001). Since PtNOA is constitutively signaling stress it was a good model for the chronic stress that phytoplankton experience in the environment due to shifting nutrients fields, UV stress, grazing pressure, viral infection. Grazing preference for healthy cells over stressed cells in the environment could lead to suppressed blooms, as mortality rates would be highest for the fastest growing cells. However, because the number of stressed cells in the phytoplankton community increases as blooms decline deterrence of microzooplankton grazers could hinder top-down control of the system, potentially prolonging blooms. Consistent with that hypothesis, diatom blooms are typically terminated by nutrient stress or aggregation and rapid sinking out of the euphotic zone, not predation (Alldredge et al., 1995; Conley and Malone, 1992). 
NO signaling played an important role in the deterrence of grazing. O. marina grazing significantly decreased when WT cells were spiked with the NO donor 5-NOC (Fig 4C). A similar decrease in grazing rates was observed when decadienal and C20:4 NVOs were added to WT + Om treatments (Figure 4D and 4F). The effects of both NO and oxylipin amendment on grazing were dose dependent (Figure 4C, 4D, and 4E). The interplay between grazing, oxylipin exposure, and NO production was further demonstrated by experiments showing significantly enhanced NO production by WT in the presences of the grazer Om and when exposed to decadienal at two different concentrations 0.2 and $5 \mathrm{uM}$ relative to the prey only, no amendment control (Figure 5). Furthermore, the addition of a NO scavenger to decadienal amended grazing experiments returned grazing rates to control levels (Figure 4D), suggesting that grazing deterrence depended on something downstream of $\mathrm{NO}$ in the stress surveillance pathway that decadienal elicits in Pt (Vardi et al., 2006).

\subsection{Lipidomic differences between healthy and stressed cells}

Since both NO and exposure to oxylipins reduced microzooplankton grazing, and both grazing and oxylipin exposure elicited NO production, the response of the lipidome to NO and grazing must be queried to understand the interdependences of this stress surveillance pathway. Overproduction of $\mathrm{NO}$ and microzooplankton grazing resulted in similar yet distinct shifts in the dissolved lipidome of Pt. The lipidomes of the stressed treatments (PtNOA, WT+Om, and PtNOA+Om) were more similar to each other than the WT (Figure 6A). Therefore, in addition to oxylipins eliciting de novo NO production (Vardi et al 2006), the reverse is also true: NO elicited de novo oxylipin production by Pt. Vardi et al. postulated this in (2008) but tied additional infochemical production in response to NO to programed cell death. However, activation of the NO stress surveillance system in PtNOA resulted in enhanced oxylipin production without cell death. In the environment this mechanism could further propagate the stress signal throughout a population using NO and oxylipins, potentially triggering downstream processes such as immunization of the cells against subsequent stress conditions.

While overproduction of NO resulted in a significant change in the dissolved lipidome, it was subtle compared to the impact of microzooplankton grazing. The two grazing treatments 
$\mathrm{WT}+\mathrm{Om}$ and PtNOA + Om formed their own significant cluster, demonstrating upregulation of more molecular species compared to PtNOA. Similarly, copepod grazing was previously shown to increase PUA production (Pohnert et al 2002). Copepods exhibit sloppy-feeding, biting into their prey, whereas, microzooplankton like $O$. marina engulf their prey whole. Despite differences in feeding style between copepods and microzooplankton, the overall impact of grazing in both cases appears to be increased oxylipin production.

Based on the relationship between stress and increased oxylipin production previously observed for PUA production in diatoms (Ribalet et al., 2009; Ribalet et al., 2007b), one might have expected the dissolved lipidome of chronically stressed PtNOA cells to respond more drastically to grazing than the healthy WT cells. However, WT cells exhibited a more statistically robust response to grazing with very significant $(\mathrm{p}<0.0167)$ upregulation of fourteen molecular species within the WT+Om lipidome compared to the WT (Figure 7). Only three molecular species were very significantly upregulated in the dissolved lipidome of grazed $\mathrm{PtNOA}(\mathrm{PtNOA}+\mathrm{Om})$ relative to the WT prey only treatment.

It was confounding that similarity profile analysis and nest t-tests showed that the dissolved lipidomes of grazed PtNOA and grazed WT were not significantly different from one another (Figure 6A), despite lower microzooplankton grazing on chronically stressed PtNOA cells and evidence from grazing experiments that oxylipin signaling played a role in grazing deterrence (Figure 4A and 4B). However, based on the dose dependent nature of oxylipin signaling, it is plausible that the few molecules significantly upregulated in PtNOA acted as chemical defense signals against microzooplankton grazing (Figure 7 and 8B).

\subsubsection{Potential microzooplankton grazing deterrents in dissolved lipidome}

An unknown lipophilic small molecule, $\mathrm{C}_{10} \mathrm{H}_{20} \mathrm{O}$ RT-2.06, was most significantly upregulated in the PtNOA prey only treatment relative to the WT treatment $(\mathrm{p}$-value $<0.0167)$ and may have served a chemical defense role by deterring microzooplankton grazing of PtNOA. Originally this molecule was annotated as decanal, a C10 saturated aldehyde previous observed in two strains of Pt that were isolated from a Norweigian Fjord (Prestegard et al., 2015). However, the $\mathrm{RT}_{\text {obs }}$ was much earlier than the RT predicted by authentic polyunsaturated aldehydes standards (Table 4h). The elemental formula matched three other molecules in the 
KEGG database: menthol, neomenthol, and citronellol (Table 5). All of these putative identifications were terpenoids, lipid molecules made up of repeating five carbon isoprene units. The majority of known natural products are terpenoids (Firn, 2010). Due to the bioactive nature of this group of compounds, I hypothesize that the $\mathrm{C}_{10} \mathrm{H}_{20} \mathrm{O}$ molecule was a terpenoid that acted as a grazing deterrent. The cyclized terpene alcohols, menthol and neomenthol, have not been reported in diatoms but citronellol, an acyclized terpene alcohol, was annotated in DiatomCYC, a database of Pt metabolism developed by mining the genome with genomic, transcriptomic, proteomic, and metabolomics resources (Fabris et al., 2012). Alcohols are more polar than aldehydes which is consistent with the early elution time of the $\mathrm{C}_{10} \mathrm{H}_{20} \mathrm{O}$ peak relative to the PUAs. Citronellol, along with other terpenes, inhibited the growth of the green alga Chlorella pyrenoidosa (Ikawa et al 1992). Menthol is known to have some anti-biofouling properties towards fungi, algae, and barnacle larvae settlement with several patents for anti-biofouling treatments that utilize menthol and related compounds (Seabrook and Stockum, 2011).

Oxylipin signaling is very dose-dependent as was demonstrated in the dose-dependent grazing experiments with decadienal (Fig 4D), HpETE (Fig4F), Leukotriene B4 (Fig 4F), and 5NOC (Fig 4C). Thus the difference in $\mathrm{C}_{10} \mathrm{H}_{20} \mathrm{O}$ concentrations between PtNOA and WT treatments may have been sufficient to deter $O$. marina grazing on chronically stressed PtNOA cells relative to WT cells. This hypothesis was supported by the divergence in the grazing rates of Om on PtNOA and WT exposed to decadienal concentrations higher than $0.2 \mu \mathrm{M}$ (Fig 4D). Between $0.5 \mu \mathrm{M}$ and $1 \mu \mathrm{M}, \mathrm{Om}$ is more severely affected by decadienal in the PtNOA treatment. I hypothesize that this is due to the compounding effects of decadienal with elevated concentrations of $\mathrm{C}_{10} \mathrm{H}_{20} \mathrm{O}$ and other small molecule elevated in PtNOA. Termed the synergist effect, multiple chain lengths of PUA are often more potent than a single compound PUA alone (Balestra et al., 2011). Furthermore, Pt was the original diatom observed to decrease copepod hatching success in the paper that presented the 'paradox of the plankton' (Ban et al. 1994). The causative agent of Pt toxicity to copepod eggs has not previously been determined as $\mathrm{Pt}$ is not known to produce PUAs. Considering the impacts of menthol on other crustaceans (i.e. barnacles), terpenoids have the potential to decrease copepod hatching success and should be investigated in future studies. 
Compounds that were released from PtNOA as it was grazed could have also deterred microzooplankton grazing. There were no molecular species significantly upregulated in the PtNOA + Om treatments compared to the WT + Om (Figure 8). However, there were some distinctly regulated molecules in the PtNOA+Om lipidome relative to the PtNOA and WT prey only lipidomes. Again given the dose dependent nature of oxylipin infochemical signaling, the small differences between the concentrations of these molecules in $\mathrm{WT}+\mathrm{Om}$ and $\mathrm{PtNOA}+\mathrm{Om}$ treatments could have been enough to deter grazing.

The molecular species significantly upregulated in the dissolved lipidome of the grazed PtNOA treatment compared to the PtNOA prey only treatment were NVO 18:5 4O RT-1.47, NVO 12:0 3O RT-1.47, and $\mathrm{C}_{10} \mathrm{H}_{14} \mathrm{O}$ RT 1.45. $\mathrm{C}_{10} \mathrm{H}_{14} \mathrm{O}$ and NVO 18:3 4O RT 1.47 were also significantly upregulated in the $\mathrm{WT}+\mathrm{Om}$ treatments relative to the prey only treatments. Therefore they likely were not microzooplankton deterrents but instead biomarkers of grazing. The molecule annotated as a 12:0 3O RT 1.47 was not significantly upregulated in the WT+Om treatment compared to the prey only treatments, representing a molecule that was uniquely upregulated upon grazing of PtNOA and a good candidate for microzooplankton deterrence.

There were nine molecular species that were significantly upregulated in the PtNOA +Om treatment compared to the WT prey only treatment. Five of these molecules were also higher in the PtNOA + Om treatment relative to the WT+Om treatment on average, suggesting that they could be the causative agents of microzooplankton grazing deterrence. NVO 22:4 40 was significantly upregulated in both grazing treatments but to a higher degree in the PtNOA+Om treatment where grazing was lower. Oxylipins with four oxygens were not previously reported in diatoms but sequential lipoxygenation of 20:4 fatty acids in red algae and in mammals haven been observed to produce dihydroperoxy acids (Brash et al., 1989; Hada et al., 1991; Jiang and Gerwick, 1997; Jiang et al., 2000; Maas et al., 1982; Serhan, 2002; Serhan et al., 1986; Yamamoto et al., 1988). The small unknown molecules $\mathrm{C}_{12} \mathrm{H}_{16} \mathrm{O}$ RT-1.59, NVO 14:2 $1 \mathrm{O}$ RT-4.38, and $\mathrm{C}_{9} \mathrm{H}_{16} \mathrm{O}$ RT-1.8 were also significantly upregulated in the PtNOA + Om relative to the WT but not in the WT + Om. These molecules did not match any of the metabolites found in the KEGG database (Table 5). These compounds could be investigated as grazing deterrents by isolating them from PtNOA + Om cultures with preparative HPLC, adding 
them to grazing experiments with WT to look for a similar decrease in grazing rate, and using derivatization and fragmentation techniques to elucidate their structure.

\subsubsection{Changes in particulate lipidome suggests grazing elicited production of C20:5 oxylipins}

The particulate oxylipidome represented the intracellular pool of free fatty acids and oxylipins. The observation of far fewer compounds in the particulate fraction compared to the dissolved (19 vs 126) is consistent with tight intracellular control of bioactive lipid molecules. Note that the relative abundance of the 20:5 free fatty acid was on average higher in the prey only WT and PtNOA treatments compared to grazed cells. This may indicate the enzymatic conversion of 20:5 to oxylipins in grazed treatments. The relative abundance of intracellular 20:5 free fatty acid was significantly higher $(\mathrm{p}<0.0167)$ in the grazed PtNOA treatment relative to grazed WT treatment suggesting a higher intracellular pool of 20:5 free fatty acid. No known 20:5 oxylipins were significantly upregulated in the dissolved or particulate lipidomes of PtNOA+Om. However, two C20:5 NVO were significantly up regulated in the dissolved lipidome of WT+Om treatments suggesting that the 20:5 oxylipin pathway may have been upregulated in the grazed WT cells resulting in a significant decrease in intracellular 20:5 free fatty acid relative to grazed PtNOA. However, the possibility that the significantly lower 20:5 free fatty acid concentrations in the $\mathrm{WT}+\mathrm{Om}$ treatment compared to the PtNOA +Om treatment was the result of the higher removal of WT cells by grazers cannot be excluded.

\subsubsection{Additional insights from the particulate lipidome}

The particulate lipidomes of the grazed treatments were more similar to each other than the prey only WT and PtNOA controls. And while prey only WT and PtNOA cells had distinct dissolved lipidomes, the particulate lipidomes were statistically the same (Figure 6A and 9A). This suggested that the basal level of oxylipin production in PtNOA cells was closely coupled to transport out of the cell into the dissolved pool such that there was no difference in intracellular oxylipidome between WT and PtNOA cells. The toxicity of oxylipins and the energetic efficiency of their use as infochemical signals have been attributed to their polarity and small 
size allowing them to diffuse/intercalate into membranes (Adolph et al., 2004). Furthermore, HPL and AOS enzymes in higher plants are chloroplast transmembrane proteins, potentially expediting exudation (Froehlich et al., 2001). Thus, close coupling between production and release would be expected.

The free fatty acids 22:6, 24:1, and 22:1 and a saturated short chain fatty acid with two oxygens (NVO 14:0-2O) were significantly more abundant in the oxylipidome of grazed PtNOA relative to the prey only controls (Fig 5B). The 14:O-2O moiety was hypothesized to be an oxidation product of a C14 intermediate in the fatty acid synthesis II pathway. Monounsaturated fatty acids are unlikely molecules for oxylipin enzymatic pathways based on LOX specificity for polyunsaturated fatty acids. The 22:1 fatty acid was observed as a minor component of the glycerol lipid fatty acids in Pt and 24:1 has been observed in trace amounts in Skeletonema and a Navicula-like diatom strain (Abida et al., 2015; Mansour et al., 2005). Therefore, I hypothesize that monounsaturated free fatty acids built up in grazed cells (although not significantly in $\mathrm{WT}+\mathrm{Om}$ ) because they were liberated from membrane lipids with PUFA but could not be shunted into oxylipin pathways.

The 22:6 fatty acid was also upregulated in the lipidome of the grazed PtNOA treatment compared to the prey-only treatments (Fig 5B). Leptocylindrus diatoms can use 22:6 free fatty acids to synthesize oxylipins and 22:6 fatty acids are also found in microzooplankton. Thus the potential contribution from the predator to the 22:6 pool cannot be overlooked. However, based on the ratio of predator to prey in the treatments (1:9) and previously published data on the grams of 22:6 fatty acid per cell in Pt (Jiang and Gao, 2004) and Om (Veloza et al., 2006), the predator was estimate to contribute one 22:6 molecule for every 5702 contributed by the prey, making it unlikely that the prey would contribute largely to the intracellular lipid pool of 22:6 free fatty acid (Equations 3-5).

\section{O. marina:}

$$
\begin{gathered}
\text { Equation (3) } \frac{\text { grams 22:6 }}{\text { gram Om biomass }} \times \frac{\text { grams carbon }}{\text { O.marina cell }}=\text { grams } 22: 6 \text { per } 10 \mathrm{~m} \mathrm{cell} \\
\frac{15.17 \mu g 22: 6}{m g C} \times \frac{5.16 \mathrm{pgC}}{\text { Om cell }}=\frac{78 \mathrm{fg} 22: 6}{\text { Om cell }}
\end{gathered}
$$




\section{P. tricornutum:}

Equation (4) $\frac{\text { grams PUFA }}{\text { Pt cell }} \times \%$ of PUFAs that are 22:6 = grams 22: 6 from 1 Pt cell

$$
\frac{9.92 \mathrm{pg} \mathrm{PUFA}}{\text { Pt cell }} \times 0.5 \%=\frac{49.6 \mathrm{pg} \mathrm{22:6}}{\text { Pt cell }}
$$

Equation (5) $\quad \frac{\text { predator }}{\text { prey }} * \frac{\text { grams } 22: 6 \text { per } 1 \text { om cell }}{\text { grams } 22: 6 \text { per } 1 \text { Pt cell }}=\frac{22: 6 \text { origniating from predator }}{22: 6 \text { originating from prey }}$

$$
\frac{1}{9} * \frac{78 f g 22: 6_{\text {predator }}}{49.6 \text { pg } 22: 6_{\text {prey }}}=\frac{1}{5702}
$$

Therefore, the 22:6 free fatty acid was likely liberated from membrane lipids when $\mathrm{Pt}$ was grazed and served as a precursor for the 22:6 NVOs that were relatively more abundant in the dissolved lipidomes of the grazed treatments. The accumulation of 22:6 in the grazed cells would suggest that lipolytic cleavage from the cell membrane and subsequent lipoxygenase was not as closely coupled as that observed in the 20:5 pathway. The turnover time of C20:5 free fatty acids may be faster than the turnover time of 22:6 free fatty acids due to the large number of enzymes in the 20:5 pathway and potentially lower affinity of oxylipin enzymes for the larger, more unsaturated 22:6 fatty acid (Halangk et al., 1977).

\subsection{Potentially bioactive molecules upregulated by grazing of WT cells}

An alternative hypothesis to the production of microzooplankton deterrents by PtNOA was that the plume of dissolved oxylipins produced by WT cells as they were grazed served as a signal to microzooplankton grazers alerting them to the presence of a high quality food source. DMSP, another secondary metabolite of phytoplankton, functioned as a strong chemoattractant for Oxyrrhis marina in microfluidic cell experiments (Seymour et al., 2010). However, the semiquantitative method used was not sufficient for testing this hypothesis and the average normalized oxy-lipidomes of grazed PtNOA and grazed WT were not distinct (Figure 7). Regardless, compounds from known diatom oxylipin pathways and several novel molecules were upregulated by grazing of WT cells and may serve some other stress signaling function in 
diatoms. Because there were so many molecules significantly upregulated in the $\mathrm{WT}+\mathrm{Om}$ treatments relative to the WT prey only treatments, I will only discuss those that were most significantly upregulated with a p-value less than 0.1 with a Bonferroni correction (p-value $<0.016$; Fig 7).

\subsubsection{C20:5 dihydroxy acids}

Di-hydroxy eicosapentaenoic acid (diHEPE) was significantly upregulated in WT+Om treatments relative to the WT prey only treatments. Various isomers of di-hydroxy octadecadienoic acid (18:2) have been described in fungi and freshwater cyanobacteria and 20:4 dihydroxy acids have been observed in red algae (Andreou et al., 2009). Di-hydroxy acids have not previously been identified in diatoms, representing a new branch in the diatom C20:5 oxylipin pathway. In the fungus Aspergillus sp., these compounds are considered precocious sexual inducer factors and was involved in the switch between asexual and sexual life cycles (Garscha et al., 2007). The function of di-hydroxy acids has not been determined in the fungus Gaeumannomyces graminis (Hamberg et al., 1994). Di-hydroxy acids prevent epiphyte settling on the red macroalgae Gracilaria chilensis (Weinberger et al., 2011) . Lastly, the human immune system di-hydroxy DHA, diHEPE, and diHETE served anti-inflammatory functions mediating cell interactions in the human immune system (Serhan et al., 2009), demonstrating the broad range of bioactivity that these functional group analogs have.

Further investigation is necessary to determine the function of di-HEPE in diatoms but grazing experiments with leukotriene B4 revealed a dose-dependent inhibition of microzooplankton grazing (Figure 4F). Leukotriene B4 is a mammalian C20:4 dihydroxy acids that is analogous to the diHEPE observed in Pt in the this study and diHETE molecules observed in red algae. Furthermore, leukotriene B4, had no impact on $(\mu)$ of Pt contrary to decadienal and HpETE (Table 1). Similar to the allelopathic effects of PUAs on marine organisms, di-HEPE may serve multiple roles in diatoms potentially impacting both diatom cell cycles and microzooplankton grazing rates. However, diHEPE wasn't significantly upregulated in the PtNOA+Om treatment so its primary role in diatom ecology may not be grazer deterrence. 
In red algae, there at four di-hydroxy acid isomers, each with a different biosynthetic pathway. Production of the 9,15-diHETE isomer was hypothesized to occur via sequential 9- and 15-LOX activity followed by immediate peroxidation of the two hydroperoxy groups to hydroxy groups (Jiang and Gerwick, 1997; Jiang et al., 2000). The 5,6-diHETE isomer was produced non-enzymatically by isomerization of 5(6)- epETE produced by AOS (Jiang et al., 2000). The 7,8-diHETE isomer has been hypothesized to be released directly from galactolipids (Weinberger et al., 2011), whereas, the 11,12-diHETE isomer was hypothesized to be produced via hydro-peroxide isomerase activity on 12(S)-HpETE. Furthermore, the gene PhLOX2 was recently cloned from the red algae Pyropia haitanensis and observed to produce an array of oxylipin substrates from C18-C22 fatty acids including 9,12-dihydroperoxy acids (4 oxygens), demonstrating the functional plasticity of oxylipin biosynthesis (Zhu et al., 2015).

Two proteins were responsible for C18 di-hydroxy acid production in fungi, linoleate diol synthase (LDS) and PpoA (Su and Oliw, 1996; Tsitsigiannis et al., 2004). In the wheat pathogen Gaeumannomyces graminis, the bi-functional enzyme linoleate diol synthase (LDS) adds a hydroperoxy group to the $8^{\text {th }}$ carbon of 18:2 free fatty acid and then isomerizes the hydroperoxy acid intermediate to the di-hydroxy acid, effectively bypassing lipoxygenation by LOX. The homologous protein Ppo A is responsible for biosynthesis of di-hydroxy octadecadienoic acid and hydroxyl octadecadienoic acid from linoleic acid in Aspergillus sp., again independent of LOX.

Diatoms are most closely related to macro red algae both falling under the taxa Chromalveolates. However, taxonomic position does not always determine homology in biosynthetic pathways. In fact, there is evidence that LOX genes have been shared by horizontal gene transfer between bacteria and between symbiotic bacteria and their hosts (Porta and RochaSosa, 2001). Along those lines, diatom diHEPE biosynthetic pathway may resemble the cyanobacterial pathway which has only be observed in vitro by incubating isolated 9-LOX/AOS fusion protein from Nostoc sp. with C18:3 free fatty acid (Lang et al., 2008). The 9-LOX is unique and thought to have evolved as a mutation of the 13-LOX common to prokaryotes (Andreou et al., 2008). With recent advances in eukaryotic genomics and the wealth of knowledge from other systems, unraveling the biosynthetic origins of these novel oxylipins in 
diatoms is low hanging fruit. Being able to approach the infochemical signaling from a genomic perspective may prove invaluable as the field progresses towards sampling the oxy-lipidomes of complex communities in the environment.

\subsubsection{Novel C18 oxylipins}

There were five C18 oxylipins upregulated in the dissolved lipidome of grazed WT treatment relative to the WT prey only treatment. Typical C18 oxylipins are produced by flowering plants and fungi from linoleic (18:2) and alpha-linolenic acids (18:3) thus these C18 oxylipins represent a novel oxylipin pathway in diatoms and potentially novel oxylipins compounds in nature. The presence of putative C18:5 oxylipins was particularly unusual because 18:5 is not observed in Pt and is often used as a biomarker for dinoflagellates (Viso and Marty, 1993). Because 18:5 free fatty acids are not observed in diatoms the contribution of prey and predator to the 18:5 pool could not be calculated as was done for the 22:6 free fatty acid. However, the presence of 18:5 oxylipins in the prey only controls suggested that 18:5 oxylipins are likely Pt derived. The strameophile Schizochytrium sp was found to use 18:5 fatty acids as an intermediate in 22:6 fatty acid production which was observed in the lipidome of Pt during this study (Ratledge, 2004). Thus 18:5 cannot be excluded as a possible trace lipid in Pt.

\subsubsection{Novel -3O oxylipins}

An oxylipin annotated as a -30 oxylipin belonging to the $\mathrm{C} 16: 4$ pathway was significantly upregulated in grazed WT treatments compared to the prey only WT. There are two classes of oxylipins with three oxygens: the 20:4 derived lipoxins and 22:6/20:5 derived resolvins. Lipoxins function as inhibitors of chemotaxis, adherence, and transmigration of neutrophil cells in the human immune system (Clària and Serhan, 1995; Fierro et al., 2003). Resolvins are anti-inflammatory molecules that return tissue to the non-inflamed state (Serhan et al., 2002). Lipoxin and resolvin biosynthesis enzymes are well characterized in humans and their production is dependent on 5-LOX activating proteins (Lehmann et al., 2015). In diatoms, 5(R)LOX leads to the production of 5-HpEPE, 5-HEPE, and 5-HepEPE thus it is possible that the 
16:4 -3O analog of lipoxin also has some dependence on 5-LOX. A study looking at resolvin production in human brain tissue, blood, and lymphocytes found non-enzymatic production of several 22:5 hydroxy epoxy acids (-2O) and tri-hydroxy acids (-3O) from the 22:6 hydroperoxy acid (Hong et al., 2003). A 16:5 PUFA does not exist in nature, so the 5-LOX dependent pathway is more likely for 16:4 30 oxylipin production than non-enzymatic production from hydroperoxy acids. However, there was a compound annotated as NVO 18:4-3O that was significantly up regulated in PtNOA prey only treatment compared to the WT prey only treatment which could be the non-enzymatic product of NVO 18:5-2O reacting with ROS (Figure 8). A focused study on -30 oxylipins that uses authentic standards to confirm these putative annotations and seeks to isolate these compounds and conduct amendment experiments will be necessary to determine the importance of these compounds. However, bioactivity of $-3 \mathrm{O}$ oxylipins in mammalian systems suggests that they are likely bioactive in Pt.

\subsubsection{C16 oxylipins}

Grazing of WT also lead to upregulation of three molecules initially thought to be components of the C16:3 and C16:4 oxylipin pathways previously described for Skeletonema sp. (d'Ippolito et al., 2004; Fontana et al., 2007a) and T. rotula (d'Ippolito et al., 2006). Predicted RTs suggested that the 16:3 1O RT- 2.78 molecules was a keto hexadecadienoic acid (KHDE) but the 16:3 1O RT- 4.33 and 16:4 3O RT-1.94 molecules could not be resolved to the functional group level. A functional group analog to KHDE, KHME (16:1 keto acid) is among the NVO produced by $S$. marinoi implicated in decreasing copepod reproductive success in cultures and in the field (Barreiro et al., 2011; Fontana et al., 2007b; Ianora et al., 2015). Thus KHDE produced by grazed Pt may serve a similar role.

\subsection{Ecological Importance}

Microzooplankton grazing and oxidative stress resulted in the significant release of several novel compounds into the dissolved organic pool (Figure 8). Structural isomers of many of these compounds are known to be bioactive in other systems implying that these molecules 
are serving infochemical signaling roles. There were equally strong lines of evidence for microzooplankton grazing deterrence by small unknown molecules upregulated initially in the PtNOA prey only treatment and different small unknown molecules upregulated by grazing of PtNOA (Figure 8). Microzooplankton grazers consume a large proportion of daily global primary productivity. Unlike copepods which are generally thought to increase particulate organic carbon export to depth via rapidly sinking encapsulated faecal pellets, microzooplankton largely increase the flow of carbon from the particulate pool to the dissolved pool by engulfing

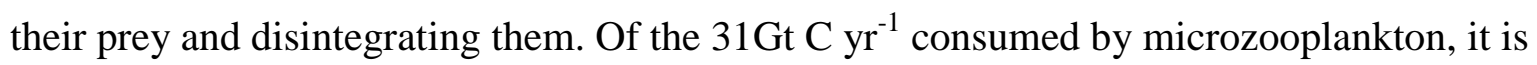
estimated that about $18 \%$ is transferred up the food web to mesozooplankton grazers like copepods and the remaining $82 \%$ is remineralized (Schmoker et al., 2013). Deterrence of microzooplankton grazing by diatoms in the environment has several ecological implications. For instance, decreased microzooplankton grazing could lead to a greater proportion of diatom primary productivity being consumed by copepods due to increased encounter rates and a potential decrease in the availability of copepod's preferred prey, flagellates and ciliates. In addition to reducing mortality of diatoms, oxylipins production may prolong blooms by stimulating recycling of nutrients by particle associated bacteria (Chapter 2).

Many of the molecules produced by grazed WT cells did not have an observed impact on grazing and are likely involved in other interactions. Oxylipins regulate sexual cycles in brown macroalgae and fungi (Andreou et al., 2009). While a sexual cycle has not been observed in Pt, these molecules may be conserved across lineages and play a role in the sexual cycles of other diatoms or life stage changes like resting spore formation, both of which occur as blooms decline (Smetacek, 2012). Resting spores tend to aggregate and sink, increasing carbon export (Rynearson et al., 2013). Sexualized diatom cells are often associated with export with some studies observing 'mass sexual phases' correlated with mass sinking of blooms (Crawford, 1995). In culture, once sexualized Ditylum brightwellii formed early post-auxospore cells and their sinking rates increased from $0.02 \mathrm{~m} \mathrm{~d}^{-1}$ to $1.4 \mathrm{~m} \mathrm{~d}^{-1}$ (Waite and Harrison, 1992). However, the cells quickly began ascending at $3 \mathrm{~m} \mathrm{~d}^{-1}$, suggesting the stressed diatom cells can switch to a sexualized phase, sink out of the upper ocean, and re-emerge later potentially in better environmental conditions. 
Furthermore, the legacy of stress in a diatom population can also impact the lipidomic response of the population to new stress. As blooms decline, autolysis of cells and concomitant production of the PUAs heptadienal and octadienal have been observed in the field (Ribalet et al., 2014). The dissolved lipidome release by a diatom population as it is grazed mid bloom may be more similar to that of the grazed WT in this study, whereas, the dissolved lipidome of a stressed diatom population at the terminus of a bloom is likely more similar to that exhibited by PtNOA.

Lastly, the production of 20:5 and 22:6 fatty acids are extremely temperature dependent in Pt, with 22:6 fatty acid synthesis only occurring at temperatures between $15^{\circ} \mathrm{C}$ and $20^{\circ} \mathrm{C}$ and 20:5 fatty acid synthesis spiking at temperatures below $10^{\circ} \mathrm{C}$ (Jiang and Gao, 2004). Therefore, sea surface temperature may influence the availability of FFA oxylipin precursors and in turn determine the bioactivity of the dissolved lipidome and microbial biogeochemistry. In addition to experiments focused on determining the bioactivity of the novel compounds observed in this study, future efforts to tease apart the controls on oxylipin production in situ will further elucidate the role of oxylipins in microbial food webs and marine biogeochemistry.

\section{Conclusion}

In conclusion, the microzooplankton grazer Oxyrrhis marina demonstrated faster grazing rates on WT cells compared to chronically stressed PtNOA cells. The stress signaling system mediated by NO was induced by both grazing and exogenous oxylipins. In turn, microzooplankton grazing was inhibited by exposure to both NO and exogenous C20:4 oxylipins. The oxylipidome of PtNOA was distinctly different from the WT, suggesting that NO elicited a stressed lipidome. Grazing resulted in a distinct upregulation in the oxylipidome. Grazing of WT cells incurred the strongest lipidomic response with upregulation of many novel oxylipins with infochemical signaling potential beyond grazing deterrence. Grazing deterrence of O. marina by PtNOA was linked to small unknown lipophilic compounds that warrant further investigation. Furthermore, DHA derived oxylipins, which were only recently discovered in diatoms, emerged as important components of the Pt lipidome. 
The focus of previous oxylipin studies in marine systems had largely reduced the ecological importance of oxylipins to the impact of PUAs on copepods. By pairing an untargeted lipidomics approach with an experimental design that looked at both grazing and overproduction of the stress signal NO, the large diversity of oxylipins produced when cells are stressed was revealed. These results suggest that chronic stress as well as environmental factors that affect membrane lipid composition can have a large impact on the bioactivity of organic molecules in the dissolved pool. The structure and enzymatic production of these new biomarkers for diatom stress must be validated and the range of their bioactivity must be determined to fully understand the role of oxylipins in regulating diatom bloom dynamics and upper ocean biogeochemistry.

\section{References}

Abida, H., Dolch, L.-J., Meï, C., Villanova, V., Conte, M., Block, M.A., Finazzi, G., Bastien, O., Tirichine, L., Bowler, C., Rébeillé, F., Petroutsos, D., Jouhet, J., Maréchal, E., 2015. Membrane Glycerolipid Remodeling Triggered by Nitrogen and Phosphorus Starvation in Phaeodactylum tricornutum. Plant Physiology 167, 118-136.

Adolph, S., Bach, S., Blondel, M., Cueff, A., Moreau, M., Pohnert, G., Poulet, S.A., Wichard, T., Zuccaro, A., 2004. Cytotoxicity of diatom-derived oxylipins in organisms belonging to different phyla, J Exp Biol, England, pp. 29352946.

Alldredge, A.L., Gotschalk, C., Passow, U., Riebesell, U., 1995. Mass aggregation of diatom blooms: Insights from a mesocosm study. Deep Sea Research Part II: Topical Studies in Oceanography 42, 9-27.

Andreou, A., Brodhun, F., Feussner, I., 2009. Biosynthesis of oxylipins in non-mammals. Progress in Lipid Research 48, 148-170.

Andreou, A.-Z., Vanko, M., Bezakova, L., Feussner, I., 2008. Properties of a mini 9R-lipoxygenase from Nostoc sp PCC 7120 and its mutant forms. Phytochemistry 69, 1832-1837.

Balestra, C., Alonso-Saez, L., Gasol, J.M., Casotti, R., 2011. Group-specific effects on coastal bacterioplankton of polyunsaturated aldehydes produced by diatoms. Aquatic Microbial Ecology 63, 123-131.

Ban, S., et al., 1997. The paradox of diatom-copepod interactions. Marine Ecology Progress Series 157, 287-293.

Barreiro, A., Carotenuto, Y., Lamari, N., Esposito, F., D'Ippolito, G., Fontana, A., Romano, G., Ianora, A., Miralto, A., Guisande, C., 2011. Diatom induction of reproductive failure in copepods: The effect of PUAs versus non volatile oxylipins. Journal of Experimental Marine Biology and Ecology 401, 13-19.

Beligni, M.V., Lamattina, L., 2001. Nitric oxide in plants: the history is just beginning. Plant, Cell \& Environment 24, 267-278.

Bligh, E.G., Dyer, W.J., 1959. A rapid method of total lipid extraction and purification. Canadian Journal of Biochemistry and Physiology 37, 911-917. 
Brash, A.R., Yokoyama, C., Oates, J.A., Yamamoto, S., 1989. Mechanistic studies of the dioxygenase and leukotriene synthase activities of the porcine leukocyte 12S-lipoxygenase. Arch Biochem Biophys 273, 414-422.

Calbet, A.a.L.M.R., 2004. Phytoplankton growth, microzooplankton grazing, and carbon cycling in marine systems. Limnology and Oceanography 49, 51--57.

Clària, J., Serhan, C.N., 1995. Aspirin triggers previously undescribed bioactive eicosanoids by human endothelial cell-leukocyte interactions. Proceedings of the National Academy of Sciences of the United States of America 92, 9475-9479.

Collins, J., Edwards, B., Fredricks, H., Van Mooy, B., in prep. A rules-based lipidomics pipeline for discovery of oxidative stress biomarkers in large datasets.

Conley, D.J., Malone, T.C., 1992. Annual cycle of dissolved silicate in Chesapeake Bay: implications for the production and fate of phytoplankton biomass. Marine ecology progress series. Oldendorf 81, 121-128.

Crawford, R.M., 1995. The role of sex in the sedimentation of a marine diatom bloom. Limnology and Oceanography 40, 200-204.

d'Ippolito, G., Cutignano, A., Briante, R., Febbraio, F., Cimino, G., Fontana, A., 2005. New C16 fatty-acid-based oxylipin pathway in the marine diatom Thalassiosira rotula. Org Biomol Chem 3, 4065-4070.

d'Ippolito, G., Cutignano, A., Tucci, S., Romano, G., Cimino, G., Fontana, A., 2006. Biosynthetic intermediates and stereochemical aspects of aldehyde biosynthesis in the marine diatom Thalassiosira rotula. Phytochemistry 67, 314 322 .

d'Ippolito, G., Tucci, S., Cutignano, A., Romano, G., Cimino, G., Miralto, A., Fontana, A., 2004. The role of complex lipids in the synthesis of bioactive aldehydes of the marine diatom Skeletonema costatum. Biochimica et Biophysica Acta (BBA) - Molecular and Cell Biology of Lipids 1686, 100-107.

Edwards, B.R., Bidle, K.D., Van Mooy, B.A.S., 2015. Dose-dependent regulation of microbial activity on sinking particles by polyunsaturated aldehydes: Implications for the carbon cycle. Proceedings of the National Academy of Sciences 112, 5909-5914.

Fabris, M., Matthijs, M., Rombauts, S., Vyverman, W., Goossens, A., Baart, G.J., 2012. The metabolic blueprint of Phaeodactylum tricornutum reveals a eukaryotic Entner-Doudoroff glycolytic pathway. Plant J 70, 1004-1014.

Fierro, I.M., Colgan, S.P., Bernasconi, G., Petasis, N.A., Clish, C.B., Arita, M., Serhan, C.N., 2003. Lipoxin A4 and aspirin-triggered 15-epi-lipoxin A4 inhibit human neutrophil migration: comparisons between synthetic 15 epimers in chemotaxis and transmigration with microvessel endothelial cells and epithelial cells. J Immunol 170, 2688-2694.

Firn, R., 2010. Nature's Chemicals. Oxford Scholarship Online.

Fontana, A., d'Ippolito, G., Cutignano, A., Miralto, A., Ianora, A., Romano, G., Cimino, G., 2007a. Chemistry of oxylipin pathways in marine diatoms. Pure and Applied Chemistry 79, 481-490.

Fontana, A., d'Ippolito, G., Cutignano, A., Romano, G., Lamari, N., Gallucci, A.M., Cimino, G., Miralto, A., Ianora, A., 2007b. LOX-induced lipid peroxidation mechanism responsible for the detrimental effect of marine diatoms on Zooplankton grazers. Chembiochem 8, 1810-1818.

Froehlich, J.E., Itoh, A., Howe, G.A., 2001. Tomato allene oxide synthase and fatty acid hydroperoxide lyase, two cytochrome P450s involved in oxylipin metabolism, are targeted to different membranes of chloroplast envelope. Plant Physiol 125, 306-317. 
Frost, B.W., 1972. Effects of Size and Concentration of Food Particles on the Feeding Behavior of the Marine Planktonic Copepod Calanus Pacificus1. Limnology and Oceanography 17, 805--815.

Garscha, U., Jerneren, F., Chung, D., Keller, N.P., Hamberg, M., Oliw, E.H., 2007. Identification of dioxygenases required for Aspergillus development. Studies of products, stereochemistry, and the reaction mechanism. J Biol Chem 282, 34707-34718.

Hada, T., Ueda, N., Takahashi, Y., Yamamoto, S., 1991. Catalytic properties of human platelet 12-lipoxygenase as compared with the enzymes of other origins. Biochim Biophys Acta 1083, 89-93.

Hamberg, M., Zhang, L.Y., Brodowsky, I.D., Oliw, E.H., 1994. Sequential oxygenation of linoleic acid in the fungus Gaeumannomyces graminis- Stereochemistry of dioxygenase and hydroperoxide isomerase reactions. Archives of Biochemistry and Biophysics 309, 77-80.

Heinbokel, J.F., 1978. Studies on the functional role of tintinnids in the Southern California Bight. I. Grazing and growth rates in laboratory cultures. Marine Biology 47, 177-189.

Hong, S., Gronert, K., Devchand, P.R., Moussignac, R.-L., Serhan, C.N., 2003. Novel Docosatrienes and 17SResolvins Generated from Docosahexaenoic Acid in Murine Brain, Human Blood, and Glial Cells: Autacoid in antiinflammation. Journal of Biological Chemistry 278, 14677-14687.

Ianora, A., Bastianini, M., Carotenuto, Y., Casotti, R., Roncalli, V., Miralto, A., Romano, G., Gerecht, A., Fontana, A., Turner, J.T., 2015. Non-volatile oxylipins can render some diatom blooms more toxic for copepod reproduction. Harmful Algae 44, 1-7.

Ianora, A., Miralto, A., Poulet, S.A., Carotenuto, Y., Buttino, I., Romano, G., Casotti, R., Pohnert, G., Wichard, T., Colucci-D'Amato, L., Terrazzano, G., Smetacek, V., 2004. Aldehyde suppression of copepod recruitment in blooms of a ubiquitous planktonic diatom. Nature 429, 403-407.

Jiang, H., Gao, K., 2004. Effects of lowering temperature during culture on the production of polyunsaturated fatty acids in the marine diatom Phaeodactylum tricornutum (Bacillariophyceae).. Journal of Phycology 40, 651-654.

Jiang, Z.D., Gerwick, W.H., 1997. Novel oxylipins from the temperate red alga Polyneura latissima: Evidence for an arachidonate 9(S)-lipoxygenase. Lipids 32, 231-235.

Jiang, Z.D., Ketchum, S.O., Gerwick, W.H., 2000. 5-Lipoxygenase-derived oxylipins from the red alga Rhodymenia pertusa. Phytochemistry 53, 129-133.

Johnson, M., Edwards, B., Beaudoin, D., Van Mooy, B.A.S., Vardi, A., submitted. Oxylipins mediate microzooplankton grazing on diatoms.

Lang, I., Goebel, C., Porzel, A., Heilmann, I., Feussner, I., 2008. A lipoxygenase with linoleate diol synthase activity from Nostoc sp PCC 7120. Biochemical Journal 410, 347-357.

Lavrentyev, P., Franzè, G., Pierson, J., Stoecker, D., 2015. The Effect of Dissolved Polyunsaturated Aldehydes on Microzooplankton Growth Rates in the Chesapeake Bay and Atlantic Coastal Waters. Marine Drugs 13, 2834.

Leflaive, J., Ten-Hage, L., 2011. Effects of 2E,4E-decadienal on motility and aggregation of diatoms and on biofilm formation. Microb Ecol 61, 363-373.

Lehmann, C., Homann, J., Ball, A.K., Blocher, R., Kleinschmidt, T.K., Basavarajappa, D., Angioni, C., Ferreiros, N., Hafner, A.K., Radmark, O., Proschak, E., Haeggstrom, J.Z., Geisslinger, G., Parnham, M.J., Steinhilber, D., Kahnt, A.S., 2015. Lipoxin and resolvin biosynthesis is dependent on 5-lipoxygenase activating protein. Faseb j. 
Li, A., Stoecker, D.K., Coats, D.W., 2001. Use of the 'Food Vacuole Content' Method to Estimate Grazing by the Mixotrophic Dinoflagellate Gyrodinium Galatheanum on Cryptophytes. Journal of Plankton Research 23, 303-318.

Maas, R.L., Turk, J., Oates, J.A., Brash, A.R., 1982. Formation of a novel dihydroxy acid from arachidonic acid by lipoxygenase-catalyzed double oxygenation in rat mononuclear cells and human leukocytes. J Biol Chem 257, 70567067.

Mansour, M., Frampton, D.F., Nichols, P., Volkman, J., Blackburn, S., 2005. Lipid and fatty acid yield of nine stationary-phase microalgae: Applications and unusual C24-C28 polyunsaturated fatty acids. Journal of Applied Phycology 17, 287-300.

Melamud, E., Vastag, L., Rabinowitz, J.D., 2010. Metabolomic Analysis and Visualization Engine for LC-MS Data. Analytical Chemistry 82, 9818-9826.

Miralto, A., Barone, G., Romano, G., Poulet, S.A., Ianora, A., Russo, G.L., Buttino, I., Mazzarella, G., Laabir, M., Cabrini, M., 1999. The insidious effect of diatoms on copepod reproduction. Nature 402, 173-176.

Nanjappa, D., Ippolito, G., Gallo, C., Zingone, A., Fontana, A., 2014. Oxylipin Diversity in the Diatom Family Leptocylindraceae Reveals DHA Derivatives in Marine Diatoms. Marine Drugs 12, 368.

Peterson, W.T.a.K.W.J., 1994. Processes controlling recruitment of the marine Calanoid copepod Temora longicornis in Long Island Sound: Egg production, egg mortality, and cohort survival rates. Limnology and Oceanography 39, 1594--1605.

Pohnert, G., 2000. Wound-Activated Chemical Defense in Unicellular Planktonic Algae. Angewandte Chemie International Edition 39, 4352--4354.

Popendorf, K.J., Fredricks, H.F., Van Mooy, B.A., 2013. Molecular ion-independent quantification of polar glycerolipid classes in marine plankton using triple quadrupole MS. Lipids 48, 185-195.

Porta, H., Rocha-Sosa, M., 2001. Lipoxygenase in bacteria: a horizontal transfer event? Microbiology-Sgm 147, 3199-3200.

Prestegard, S.K., Erga, S.R., Steinrucken, P., Mjos, S.A., Knutsen, G., Rohloff, J., 2015. Specific Metabolites in a Phaeodactylum tricornutum Strain Isolated from Western Norwegian Fjord Water. Mar Drugs 14.

Ratledge, C., 2004. Fatty acid biosynthesis in microorganisms being used for Single Cell Oil production. Biochimie $86,807-815$.

Ribalet, F., Bastianini, M., Vidoudez, C., Acri, F., Berges, J., Ianora, A., Miralto, A., Pohnert, G., Romano, G., Wichard, T., Casotti, R., 2014. Phytoplankton Cell Lysis Associated with Polyunsaturated Aldehyde Release in the Northern Adriatic Sea. PLoS ONE 9, e85947.

Ribalet, F., Berges, J.A., Ianora, A., Casotti, R., 2007a. Growth inhibition of cultured marine phytoplankton by toxic algal-derived polyunsaturated aldehydes. Aquatic Toxicology 85, 219-227.

Ribalet, F., Intertaglia, L., Lebaron, P., Casotti, R., 2008. Differential effect of three polyunsaturated aldehydes on marine bacterial isolates. Aquatic Toxicology 86, 249-255.

Ribalet, F., Vidoudez, C., Cassin, D., Pohnert, G., Ianora, A., Miralto, A., Casotti, R., 2009. High plasticity in the production of diatom-derived polyunsaturated aldehydes under nutrient limitation: physiological and ecological implications. Protist 160, 444-451.

Ribalet, F., Wichard, T., Pohnert, G., Ianora, A., Miralto, A., Casotti, R., 2007b. Age and nutrient limitation enhance polyunsaturated aldehyde production in marine diatoms. Phytochemistry 68, 2059-2067. 
Rynearson, T.A., Richardson, K., Lampitt, R.S., Sieracki, M.E., Poulton, A.J., Lyngsgaard, M.M., Perry, M.J., 2013. Major contribution of diatom resting spores to vertical flux in the sub-polar North Atlantic. Deep-Sea Research Part I-Oceanographic Research Papers 82, 60-71.

Schmoker, C., Hernandez-Leon, S., Calbet, A., 2013. Microzooplankton grazing in the oceans: impacts, data variability, knowledge gaps and future directions. Journal of Plankton Research 35, 691-706.

Seabrook, S.G., Stockum, G., 2011. Polymer coatings containing phytochemical agents and methods for making and using same. Google Patents.

Serhan, C.N., 2002. Lipoxins and aspirin-triggered 15-epi-lipoxin biosynthesis: an update and role in antiinflammation and pro-resolution. Prostaglandins Other Lipid Mediat 68-69, 433-455.

Serhan, C.N., Hamberg, M., Samuelsson, B., Morris, J., Wishka, D.G., 1986. On the stereochemistry and biosynthesis of lipoxin B. Proc Natl Acad Sci U S A 83, 1983-1987.

Serhan, C.N., Hong, S., Gronert, K., Colgan, S.P., Devchand, P.R., Mirick, G., Moussignac, R.L., 2002. Resolvins: a family of bioactive products of omega-3 fatty acid transformation circuits initiated by aspirin treatment that counter proinflammation signals. J Exp Med 196, 1025-1037.

Serhan, C.N., Yang, R., Martinod, K., Kasuga, K., Pillai, P.S., Porter, T.F., Oh, S.F., Spite, M., 2009. Maresins: novel macrophage mediators with potent antiinflammatory and proresolving actions. J Exp Med 206, 15-23.

Seymour, J.R., Simó, R., Ahmed, T., Stocker, R., 2010. Chemoattraction to Dimethylsulfoniopropionate Throughout the Marine Microbial Food Web. Science 329, 342-345.

Smetacek, V., 2012. Making sense of ocean biota: How evolution and biodiversity of land organisms differ from that of the plankton. Journal of Biosciences 37, 589-607.

Stoecker, D.K., Nejstgaard, J.C., Mashusoodhanan, R., Pohnert, G., Wolfram, S., Jakobsen, H.H., Šulčius, S., and Larsen, A., 2015. Underestimation of microzooplankton grazing in dilution experiments due to inhibition of phytoplankton growth. Limnology and Oceanography 60, 1426--1438.

$\mathrm{Su}, \mathrm{C}$., Oliw, E.H., 1996. Purification and Characterization of Linoleate 8-Dioxygenase from the Fungus Gaeumannomyces graminis as a Novel Hemoprotein. Journal of Biological Chemistry 271, 14112-14118.

Tsitsigiannis, D.I., Zarnowski, R., Keller, N.P., 2004. The lipid body protein, PpoA, coordinates sexual and asexual sporulation in Aspergillus nidulans. J Biol Chem 279, 11344-11353.

Vardi, A., Bidle, K.D., Kwityn, C., Hirsh, D.J., Thompson, S.M., Callow, J.A., Falkowski, P., Bowler, C., 2008. A diatom gene regulating nitric-oxide signaling and susceptibility to diatom-derived aldehydes. Curr Biol 18, 895-899.

Vardi, A., Formiggini, F., Casotti, R., De Martino, A., Ribalet, F., Miralto, A., Bowler, C., 2006. A Stress Surveillance System Based on Calcium and Nitric Oxide in Marine Diatoms. PLoS Biol 4, e60.

Veloza, A., Chu, F.-L., Tang, K., 2006. Trophic modification of essential fatty acids by heterotrophic protists and its effects on the fatty acid composition of the copepod Acartia tonsa. Marine Biology 148, 779-788.

Viso, A.-C., Marty, J.-C., 1993. Fatty acids from 28 marine microalgae. Phytochemistry 34, 1521-1533.

Waite, A., Harrison, P.J., 1992. Role of sinking and ascent during sexual reproduction in the marine diatom Ditylum brightwelli. Marine Ecology Progress Series 87, 113-122. 
Weinberger, F., Lion, U., Delage, L., Kloareg, B., Potin, P., Beltran, J., Flores, V., Faugeron, S., Correa, J., Pohnert, G., 2011. Up-Regulation of Lipoxygenase, Phospholipase, and Oxylipin-Production in the Induced Chemical Defense of the Red Alga Gracilaria chilensis against Epiphytes. Journal of Chemical Ecology 37, 677-686.

Wichard, T., Poulet, S.A., Pohnert, G., 2005. Determination and quantification of $\alpha, \beta, \gamma, \delta$-unsaturated aldehydes as pentafluorobenzyl-oxime derivates in diatom cultures and natural phytoplankton populations: application in marine field studies. Journal of Chromatography B 814, 155-161.

Yamamoto, S., Ueda, N., Ehara, H., Maruyama, T., Yokoyama, C., Kaneko, S., Yoshimoto, T., Komatsu, N., Watanabe, K., Hattori, A., et al., 1988. Biochemical studies on mammalian lipoxygenases. Ann N Y Acad Sci 524, 12-26.

Zhu, Z., Qian, F., Yang, R., Chen, J., Luo, Q., Chen, H., Yan, X., 2015. A Lipoxygenase from Red Alga Pyropia haitanensis, a Unique Enzyme Catalyzing the Free Radical Reactions of Polyunsaturated Fatty Acids with Triple Ethylenic Bonds. Plos One 10. 
Figure 1. Diatom oxylipin biosynthesis pathways from the literature.

A) Hexadecatrienoic acid pathway. Lipoxygenation of the C16:3 free fatty acid (HTrE; 1$)$ at the 6th and 9th carbons yields 6-hydroperoxy hexadecatrienoic acid (6-HpHTrE; 2) and 9-HpHTrE (3). HPL cleaves 6-HpHTrE to produce two hydroxyl acids, 6-hydroxy octenoic acid (4) and 8- hydroxy octenoic acid (5). Hydroperoxy lyase (HPL) can cleave 9-HpHTrE to produce the PUA octadienal (6) and 8-hydroxy octenoic acid (5). Allene oxide synthase (AOS) produces 11,9- hydroxyepoxy hexadecadienoic acid (11,9-HepHDE;7) from 9-HpHTrE (3). Peroxidase activity reduces both 6- and 9- HpHTrE to the respective hydroxy acid, 6-HHTrE (8) and 9-HHTrE (9). 6-HpHTrE has also been observed to non-enzymatically covert to the keto acid 6-KHTrE (10).

B) Hexadecatetraenoic acid pathway. LOX enzymes add a hydroperoxyl group to the 6th and 9th carbon in the C16:4 free fatty acid (HTE; 11) forming 6-hydroperoxy hexatetraenoic acid (6-HpHTE; 12) and 9- HpHTE (13). HPL activity produces the PUA octatrienal (14) and 8-hydroxy octenoic acid (5) from 9-HpHTE (13). Similar to the C16:3 pathway, peroxidase activity reduces both 6- and 9- HpHTE to the respective hydroxy acids, 6-hydroxy hexadecatetranoic acid (6-HTE; 15) and 9-HHTE (16). 6-HpHTrE (12) has also been observed to non-enzymatically covert to 6-keto hexadecatetraenoic acid (6-KHTE; 17).

C) Arachidonic acid pathway (AA;18). Activity of LOX enzymes specific for the 11th and 12th carbons of the C20:4 free fatty acid yields (11R)-hydroperoxy eicosatetraenoic acid (HpETE;19) and 12-HpETE (20). HPL cleaves 11R-HpETE (19) to produce the PUA decadienal (21) and 10-hydroxy decadienoic acid (22), whereas, the peroxidase activity results in 11-HETE (23) production. Hydroperoxide halolyase (HPH) cleaves 12-HpETE (20) to produce 12-oxo dodecadienoic acid (24) and chlorinated alkenes (25).

D) Eicosapentaenoic acid pathway (EPA;26). LOX enzymes targeting the 5th, 9th, 11th, 12th, 14th, 15th, or 18th carbons of the C20:5 free fatty acid produce the respective hydroperoxy eicosapentaenoic acids (5-, 9-, 11R-, 12-, 14-, 15-, 18-HpETE; 27-33). HPL cleaves 9-HpETE (28) to produce 9-oxo nondienoic acid (34) and diatom hormones finavarrene (35) and hormosirene (36), 11R-HpEPE (29) to produce the PUA decatrienal (37) and 10hydroxy decadienoic acid (24), and 14-HpEPE (31) to produce the PUA heptadienal (38) and 13-hydroxy tridecatrienoic acid (39). AOS acts on 5-HpEPE (27) to produce 7,5 HepETE (40), 9-HpEPE (28) to produce 7,8HepETE (41), 14-HpEPE (31) to produce 13,14-HepETE (42) and 16,14 HepETE (43), 15-HpEPE (32) to produce 15,16 HepETE(44), and 18-HpEPE (33) to produce 16,17 HpETE (45). Peroxidase activity produces 5-, 9-, 11R-, 14-, 15-and 18-HEPE (46-51) from the respective HpEPE precursors. HPH activity cleaves 12-HpEPE (30) to produce 12-oxo dodecatrienoic acid (25) and a chlorinated alkene (52)

E) Docosahexenoic acid pathway (DHA; 53). LOX enzymes specific to the 17th and 20th carbon on the C22:6 free fatty acid produce 17- hydroperoxy docosahexenoic acid (17-HpDoHE; 54) and 20-HpDoHE (55). Peroxidation of 17- and 20-HpDoHE produces 17-hydroxy docosahexenoic acid (17-HDoHE; 56) and 20-HDoHE (57). Activity of AOS yields 15,16 hydroxy epoxy docosapentaenoic acid (15,16-HepDoPE; 58) from 17-HpDoHE (54) and 18,19HepDoPE (59) from 20-HpDoHE (55).

Adapted from Andreou et al. (2009) and Nanjappa et al. (2014). Note this figure does not encompass the entire diversity of molecules produced by diatoms. Additionally this figure does not reflect the chirality or the cis-trans orientations of bonds. 


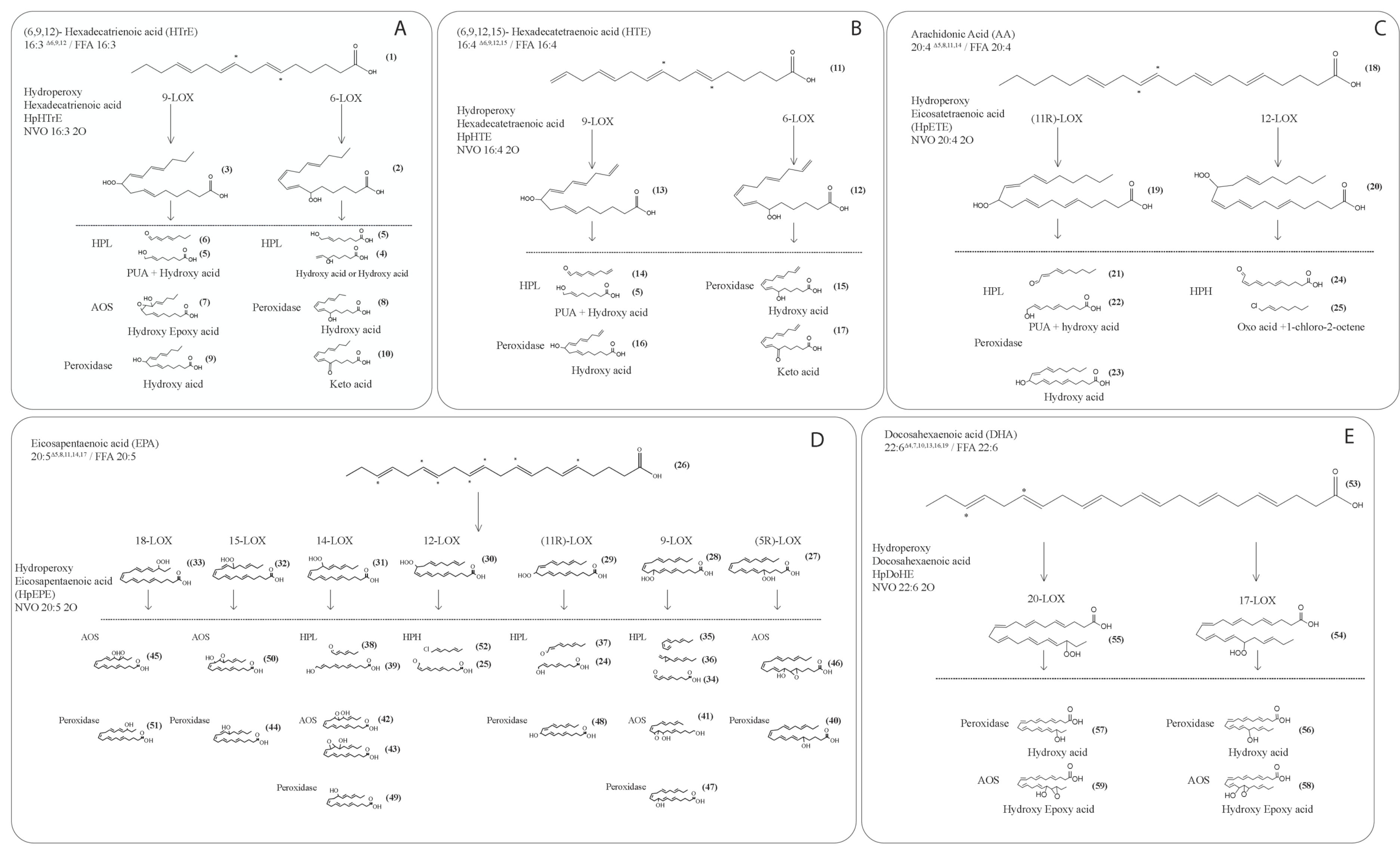




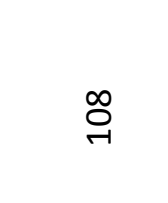



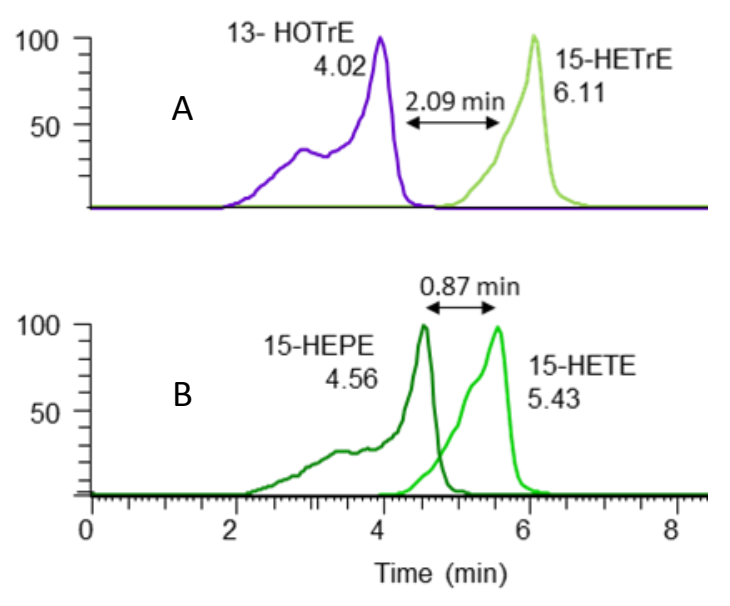

Figure 2. A) Extracted ion chromatogram (EIC) of 13-HOTrE and 15-HETrE demonstrating the 2.09 min increase in retention time associated with the addition of two carbons. B) EIC of 15HEPE and 15-HETE demonstrating the 0.87 min increase in retention time associated with C20 oxylipins losing one double bond. These offsets represent the basis of functional group annotation of molecules that included in the authentic standard set. 


\section{Structural Level Annotation (ex: NVO, PUA, FFA)}

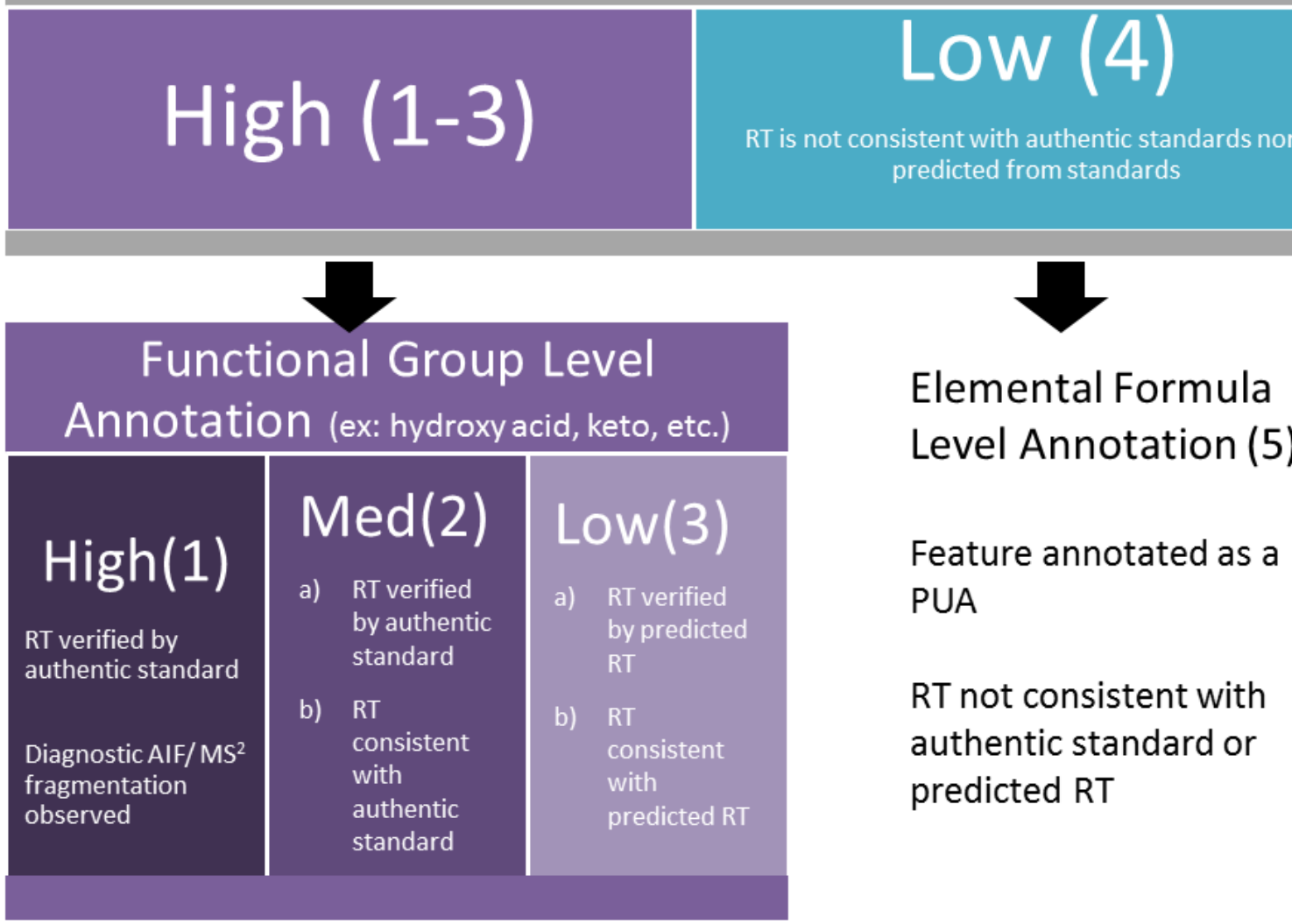

Figure 3. Confidence ranking system for putative annotations. MAVEN detected features and queried the $\mathrm{m} / \mathrm{z}$ of these features against a custom database resulting in structural level annotations. The retention times (RT) of these features were then compared to the RT of the authentic standards or RT predicted from authentic standards to resolve the features in the dissolved lipidome to the functional group level. High confidence structural annotations were denoted by a number 1-3. Level 1 confidence rankings were verified by diagnostic fragmentation in addition to having a RT consistent with an authentic standard. There were no Level 1 annotations in this data set because all ion fragmentation (AIF) was used and was not selective enough to generate diagnostic fragments. Level 2 confidence rankings were assigned to features that had a RT in agreement with an authentic standard. The letter "a" denotes that the RT observed for the feature ( $\mathrm{RT}_{\text {obs }}$ ) was an exact match to the RT of the authentic standard (RT $\left.\mathrm{Ruth}_{\text {ath }}\right)$. The letter "b" denotes that the $\mathrm{RT}_{\text {obs }}$ was within 0.7 min of the $\mathrm{RT}_{\text {auth }}$ for $-2 \mathrm{O}$ NVOs or 0.5 min of the RT $\mathrm{Ruth}_{\text {ath }}-1 \mathrm{O}$ NVOs, fatty acids, and PUAs. Level 3 confidence rankings required that the $\mathrm{RT}_{\text {obs }}$ was in agreement with $\mathrm{RT}$ predicted from authentic standards. Level 4 confidence rankings had an $\mathrm{RT}_{\mathrm{obs}}$ that did not match the RTs proposed by the suite of authentic standards. The level 4 confidence ranking represented a proposed structural level annotation consistent with oxylipin subclasses (i.e. non-volatile oxylipins, PUAs, and free fatty acids). Level 5 annotations represented unknown compounds that had an elemental formula determined by exact mass but were excluded from the proposed oxylipin subclass based on RT. The level 5 annotations in this study were compounds originally annotated as PUAs but eluted much earlier than the PUA standards would predict thus the structural level annotation was rejected and the features were assigned an elemental formula level annotation. 
Figure 4. Impacts of NO production and oxylipins on microzooplankton grazing.

A) Instantaneous ingestion rates of Oxyrrhis marina feeding on wild-type Phaeodactulym tricornutum (WT), a transgenic control (PtGFP), wild-type cells exposed to the external NO donor NOC-5, and a transgenic strain that over produces NO (PtNOA). Values represent an average over triplicate treatments. Error bars represent standard deviation from the mean. Asterisks denote significant difference from the WT control.

B) Density dependent grazing of Om on wild-type cells (WT; black circles) and chronically stressed cells (PtNOA; white circles). Ingestion rates shown for increasing prey-to-predator ratios for experiments with PtNOA and WT. Error bars show standard deviation from the mean ( $\mathrm{N}=3$ for each treatment).

C) Dose-dependent response of microzooplankton grazing to NO. Ingestion rates of WT being fed on by Om at various concentrations of the NO donor NOC-5.

D) Dose-dependent response in microzooplankton grazing to decadienal. Average normalized ingestion rates of WT (black) and PtNOA (white) by Om with increasing concentrations of decadienal. Values represent an average across each treatment $(\mathrm{N}=3)$ Error bars denote significant deviation from the control.

E) The NO mediated impact of decadienal on grazing. Ingestion rates of WT by Om across 4 treatments with decadienal ( $0,0.05 \mu \mathrm{M}, 0.5 \mu \mathrm{M}$, and $5 \mu \mathrm{M})$ with (+CPTIO) and without (control) a NO scavenger. Values represent an average across triplicates with error bars showing the standard deviation from the mean. Asterisks denote significant difference from the respective control treatment.

F) Dose-dependent impact of 20:4 oxylipins on microzooplankton grazing. Control normalized averaged ingestion rate of WT by Om in treatments amended with varying concentrations of 15HpETE (black circle) and Leukotriene B4 (di-HPETE; white circle). Values denote average across triplicate treatments. Error bars show standard deviation from the mean. 

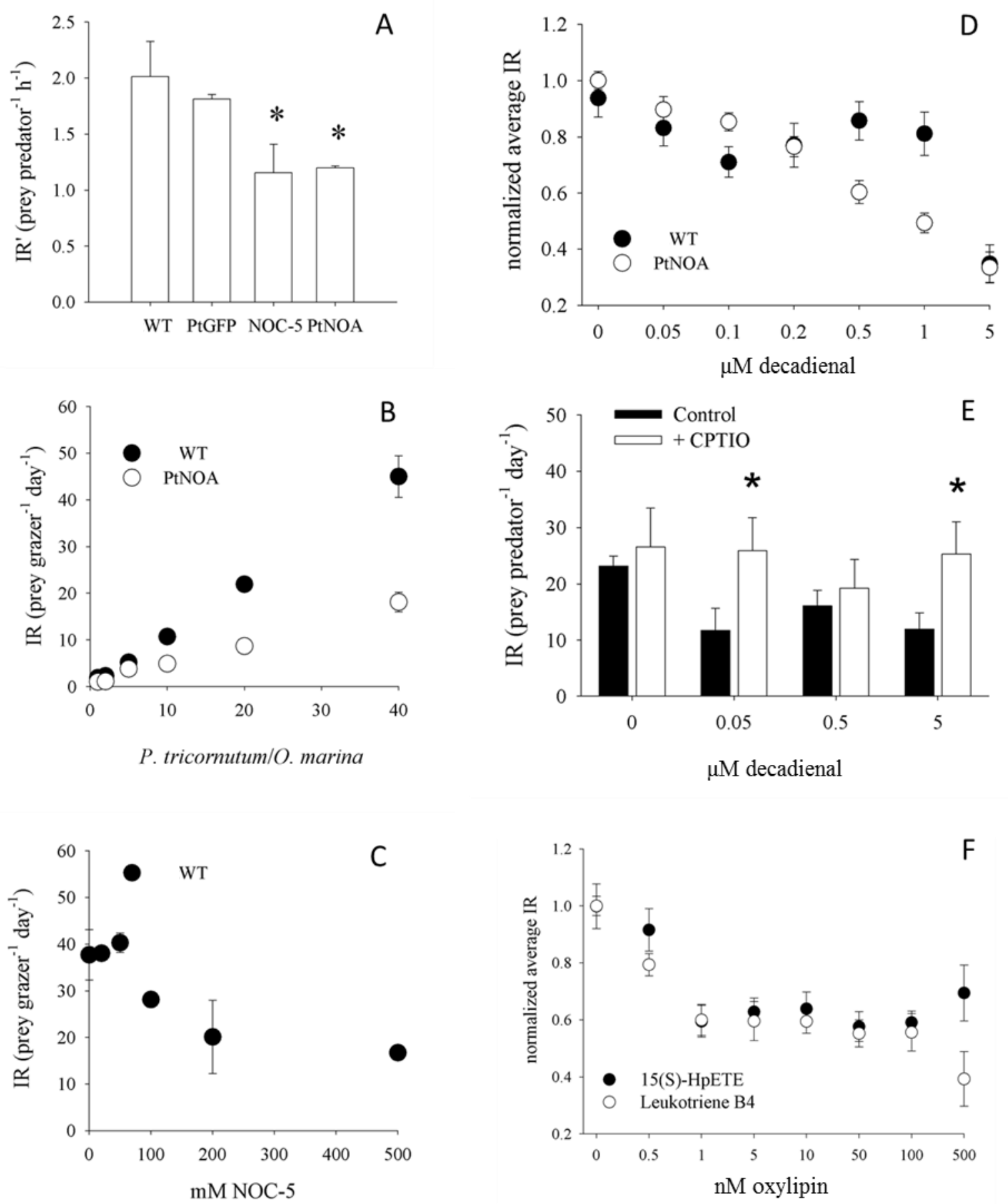


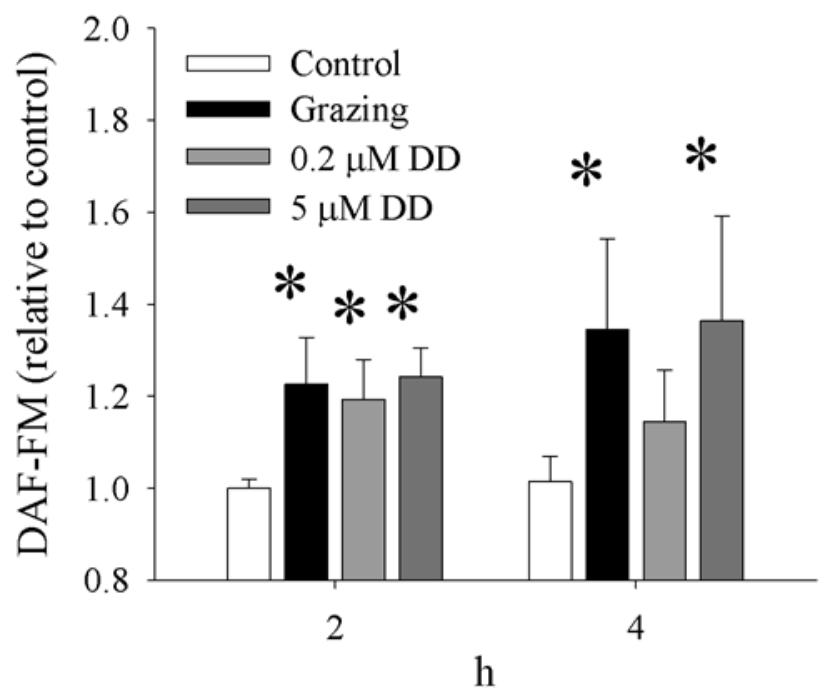

Figure 5. Microzooplankton grazing and exposure to decadienal both upregulate internal NO production in wild-type Phaeodactylum tricornutum. Control normalized DAF-FM fluorescence, a measure of the internal concentrations of NO, in wild-type cells (control), wild-type cells grazed by Oxyrrhis marina (grazing), and wild-type cells exposed to $0.2 \mathrm{uM}$ and $5 \mathrm{uM}$ decadienal (DD). Values represent control normalized averages across each treatment. Error bars show standard deviation from the mean. Asterisks denote significant difference from the control treatment. 


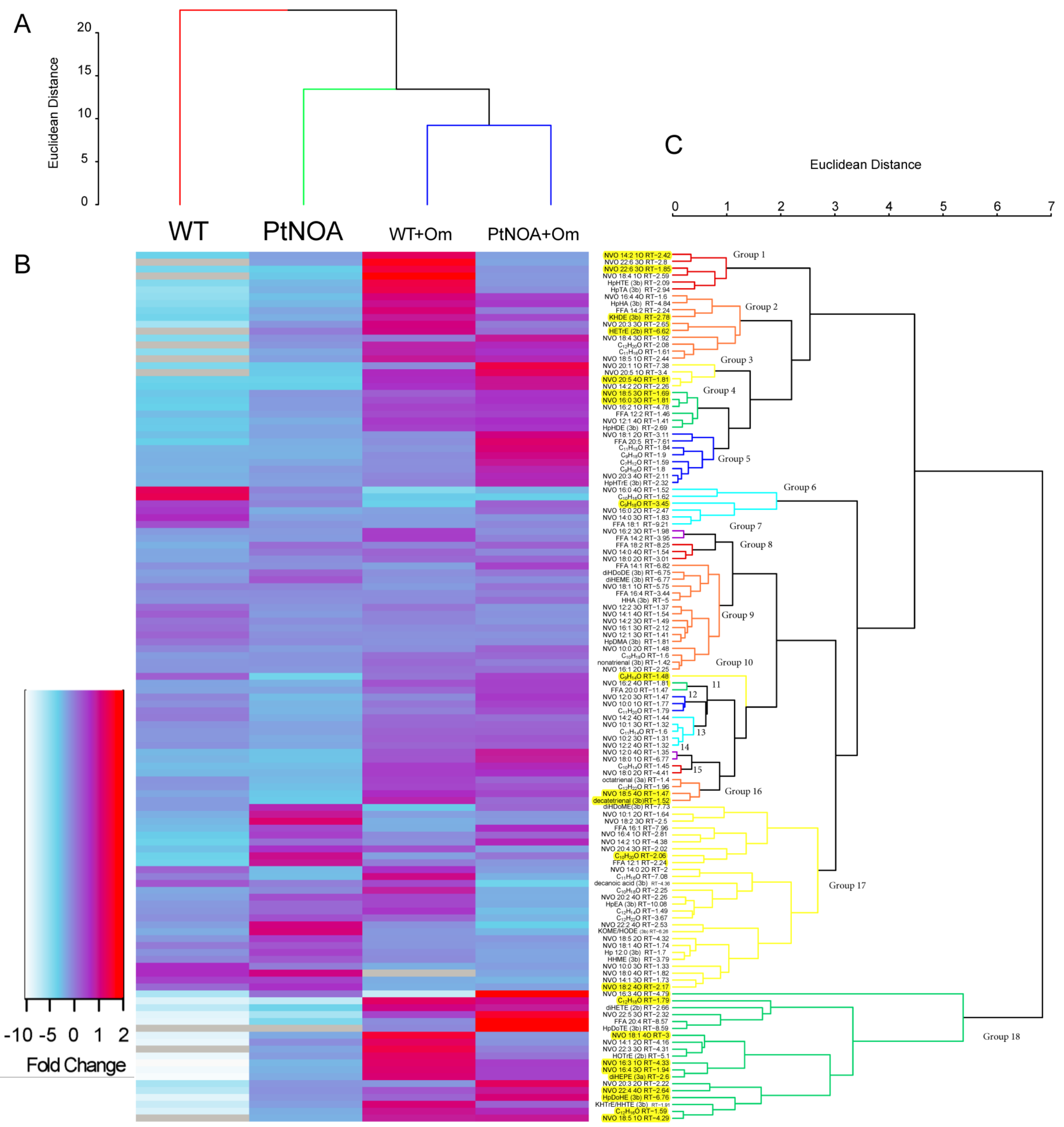

Figure 6. Dissolved lipidome of Phaeodactylum tricornutum WT and PtNOA cells with and without the grazer Oxyrrhis marina. (A) Similarity profile analysis of the four treatments based on the distribution of free fatty acids and oxylipins. WT (red) was significantly different from the PTNOA (green) and grazing treatments (blue). (B) Heat map of the average fold change of free fatty acids and oxylipin derivatives within each treatment relative to the averaged peak area across all treatments. (C) Similarity profile analysis of each molecular species based on its relative peak area across the treatments. There were 18 significantly clustering groups identified. Annotations highlighted varied most significantly between treatments (p-value $<0.0167$; Figure 7). 

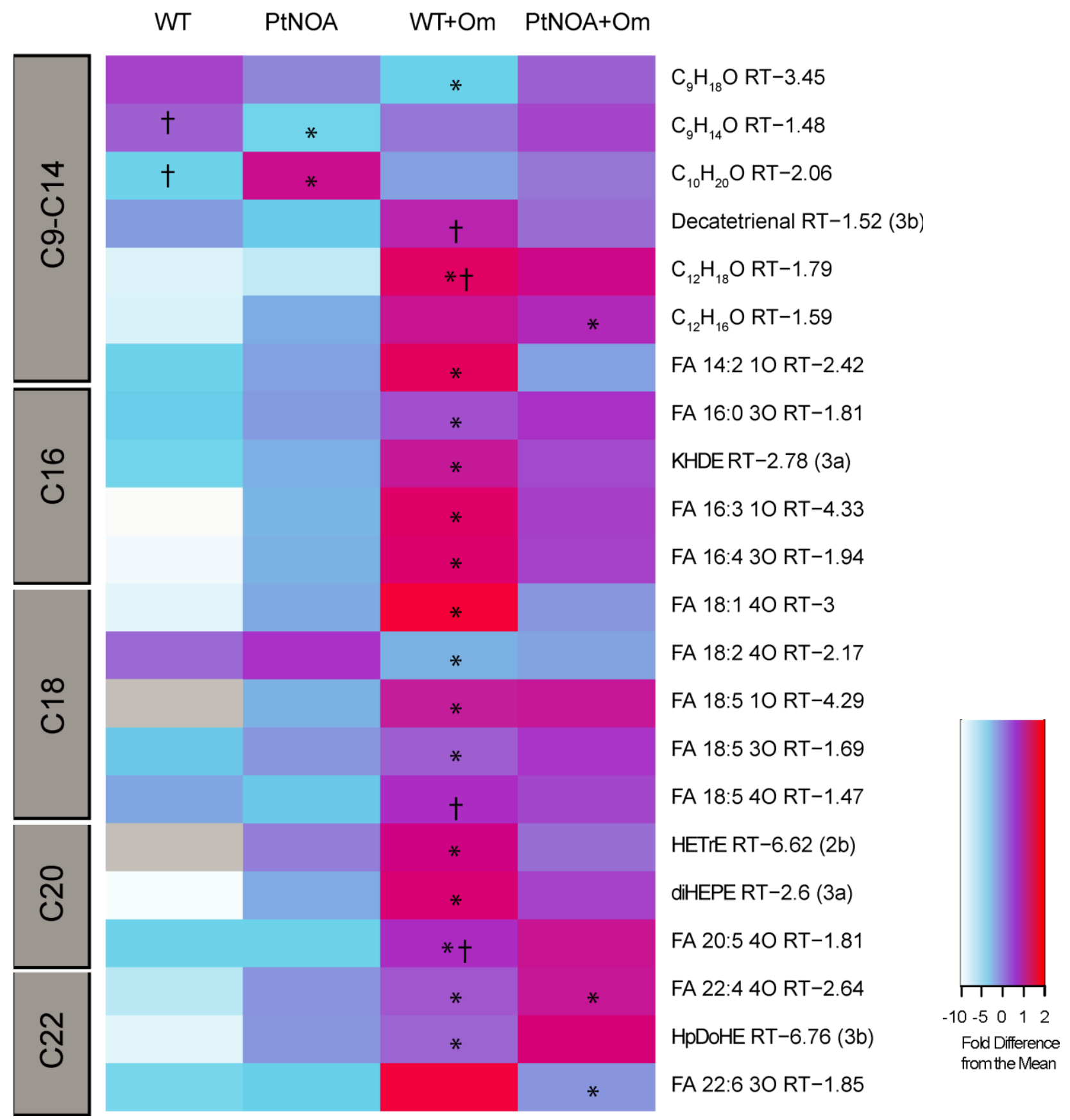

Figure 7. Heat map of the average fold change of each molecule relative to the averaged peak area across the four treatments with the molecular species most significantly regulated in the dissolved lipidome of Phaeodactylum tricornutum by chronic stress (PtNOA), grazing (WT+Om), or a combination of chronic stress and grazing (PtNOA+Om). Asterisks denote significant difference from the WT control and crosses denote significant difference from the prey only PtNOA treatments (t-test with Bonferroni correction; p-value <0.0167). Molecular species were group according to carbon chain length (grey boxes). 
A
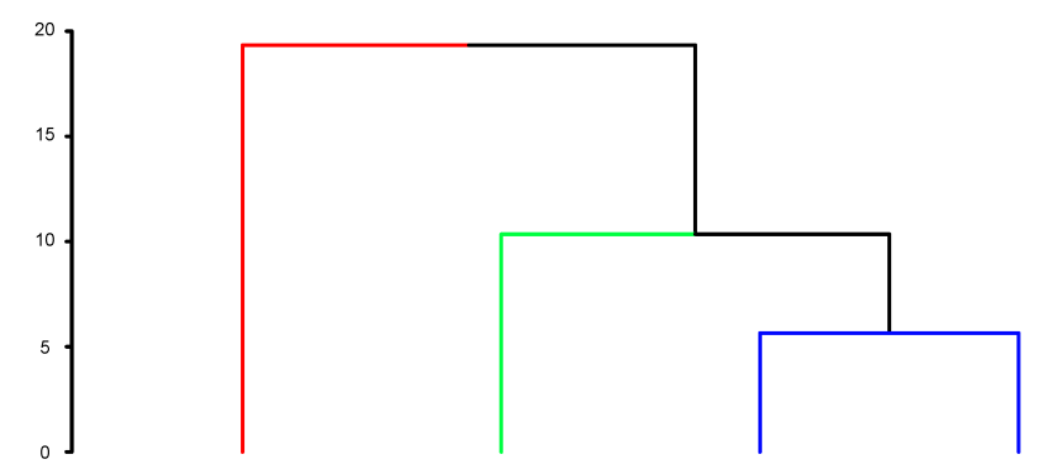

C

B

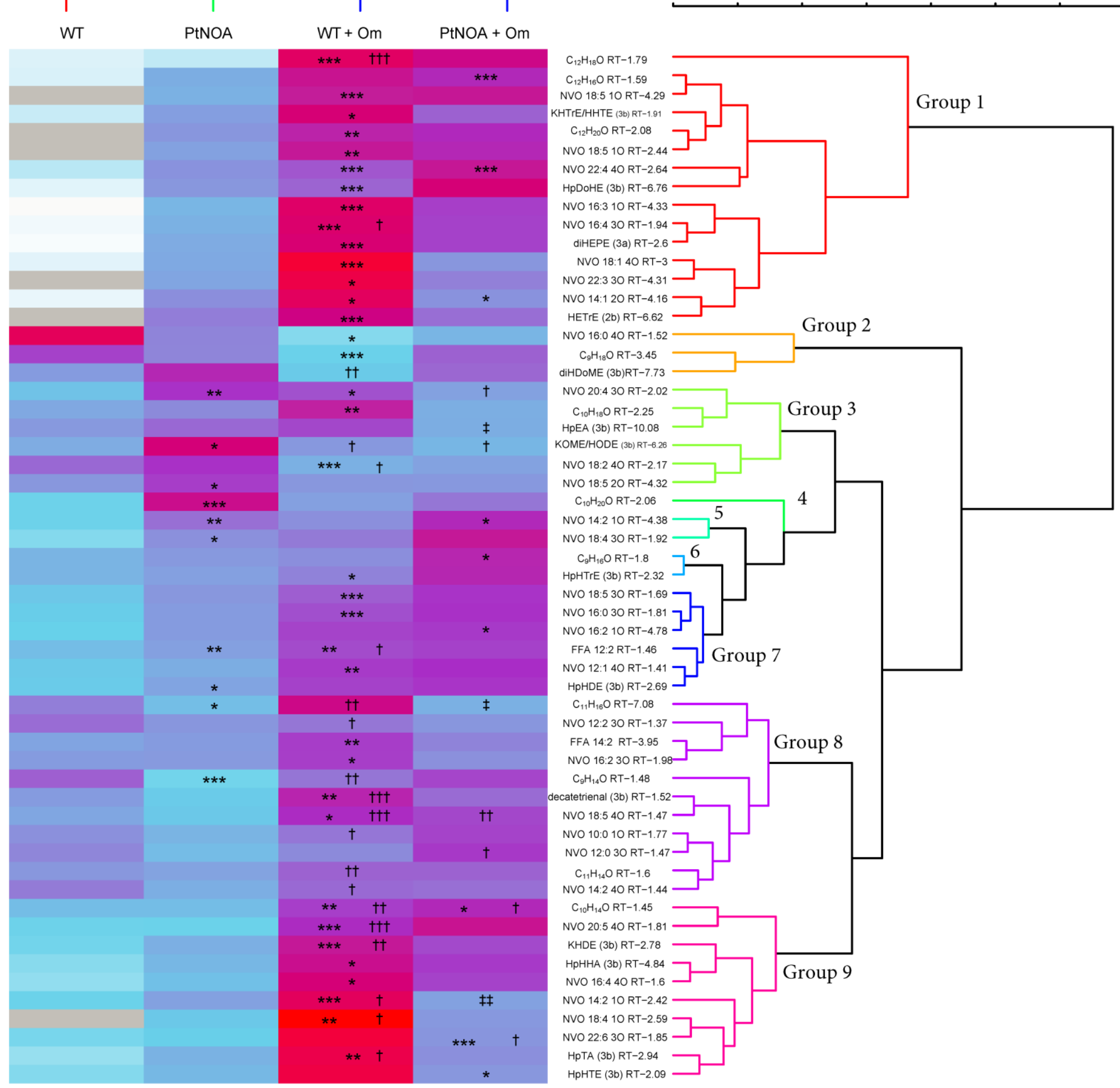

Figure 8. Expanded view of the molecular species significantly regulated in the dissolved lipidome of Phaeodactylum tricornutum by chronic stress (PtNOA), grazing (WT+Om), or a combination of chronic stress and grazing (PtNOA+Om). A) Similarity profile analysis of the four treatments based on the distribution of free fatty acids and oxylipins. WT (red) is significantly different from the PTNOA (green) and grazing treatments (blue). B) Heat map of the average fold change of each molecule relative to the averaged peak area across the four treatments. Asterisks denote significant difference from the WT control, crosses denote significant difference from the prey only PtNOA treatments, and double daggers denote significant difference from the WT+Om treatments. One symbol denotes a significant difference at a $p$-value $<0.1$. Two symbols denote significant difference at a $p$-value $<0.05$. Three symbols denote the most significant differences at a p-value $<0.0167$, representing a Bonferroni correction on a $p$-value $=0.1$. The most significantly varying compounds can be found in Figure 7. C) Similarity profile analysis of each molecular species based on the relative peak area across the treatments. There were 9 significantly clustering groups identified. 
A

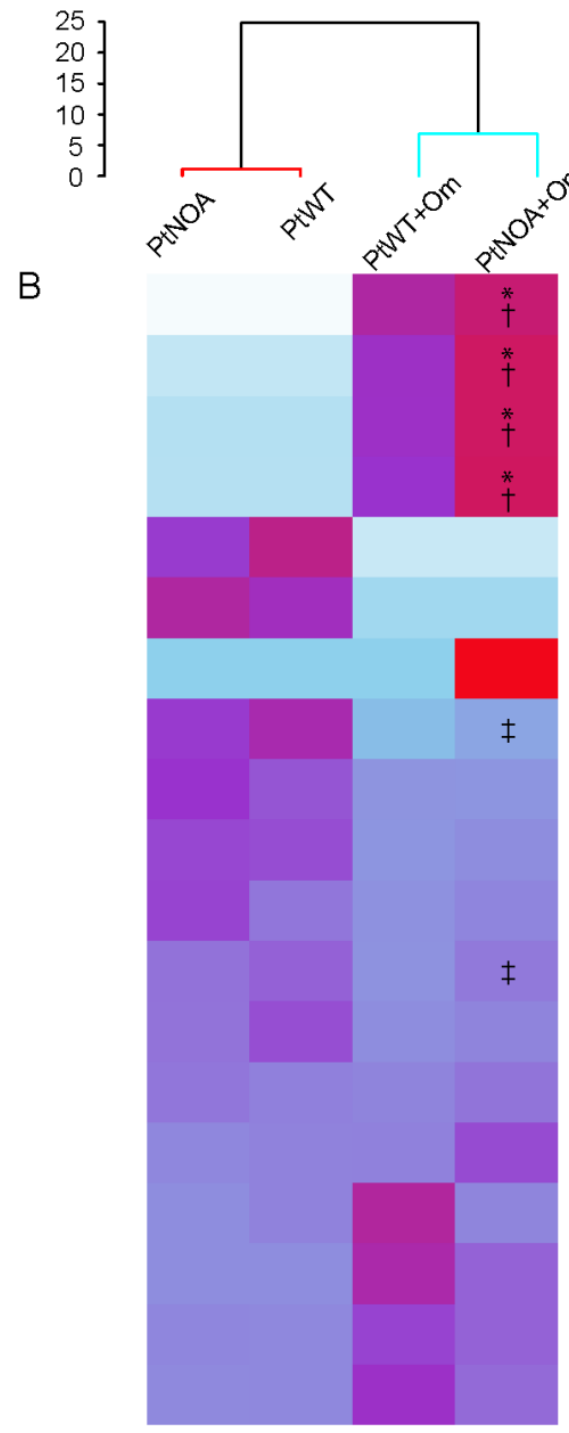

C

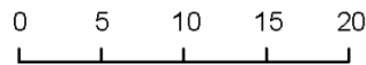

FA 22:6 none RT- 7.8

FA 14:0 20 RT- 9.3

FA 22:1 none RT- 11

FA 24:1 none RT- 12.6

FA 16:2 none RT- 7.4

FA 18:2 20 RT- 4.9

FA 16:1 30 RT- 3.8

FA 20:5 none RT- 7.6

FA 22:1 2O RT- 9.3

FA 24:1 2O RT- 10.5

FA 26:1 2O RT- 12

FA 12:2 10 RT- 3.2

FA 26:0 none RT- 17.1

FA 22:0 none RT- 12.6

FA 24:0 none RT- 14.7

FA 10:1 10 RT- 3.5

FA 20:0 20 RT- 9.5

FA 14:2 2O RT- 4

FA 20:0 none RT- 10.9
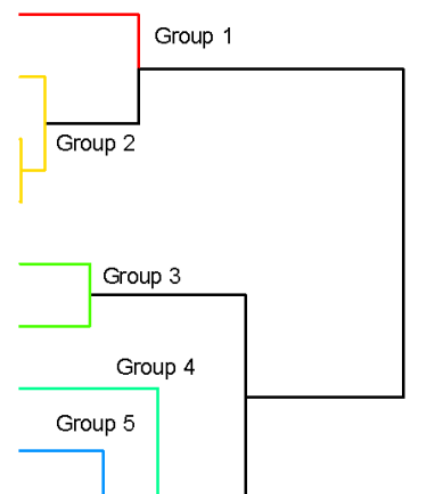

Group 6
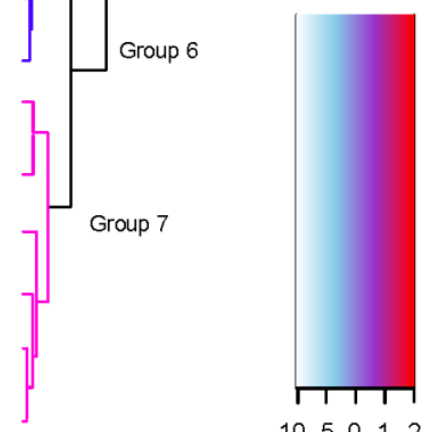

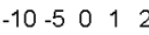

Fold Change

Figure 9. Particulate lipidome of Phaeodactylum tricornutum WT and PtNOA cells with and without the grazer Oxyrrhis marina (Om). (A) Similarity profile analysis of the four treatments based on the distribution of free fatty acids and oxylipins. WT (red) is significantly different from the other treatments (cyan). (B) Heat map of the average fold change of free fatty acids and oxylipin derivatives within each treatment relative to the averaged peak area across all treatments. Asterisks denote significant difference from the WT control, crosses denote significant difference from the prey only PtNOA treatments, and double daggers denote significant difference from WT + Om treatment ( $\mathrm{t}$-test with Bonferroni correction; $\mathrm{p}$-value $<0.0167$ ). (C) Similarity profile analysis of each molecular species based on its relative peak area across the treatments. There were 7 significantly clustering groups identified. 
Table 1. Growth rate $(\mu)$ of Oxyrrhis marina and Phaeodactylum tricornutum no predator controls in experiments with additions of the decadienal, 15(s)HpETE, and leukotriene B4. Decadienal experiments were conducted with wild type strain (WT) and a transgenic strain overexpression a nitric oxide-associated protein (PtNOA). Experiments with 15(s)-HpETE and leukotriene B4 were only conducted with WT. Numbers in bold indicate significant differences, as determined by an ANOVA with HSD $(\mathrm{p}<0.05)$.

\begin{tabular}{|c|c|c|c|c|c|}
\hline \multirow[b]{3}{*}{ Exp No. } & \multirow[b]{3}{*}{$\begin{array}{l}\text { Oxylipin } \\
(\mu \mathrm{M})\end{array}$} & \multicolumn{4}{|c|}{ Growth Rate $(\mu)$} \\
\hline & & \multicolumn{2}{|c|}{ O. marina } & \multicolumn{2}{|c|}{ P. tricornutum } \\
\hline & & Mean & SD & Mean & $\mathrm{SD}$ \\
\hline \multicolumn{6}{|c|}{ WT + decadienal } \\
\hline 1 & 0 & 0.46 & 0.31 & 0.41 & 0.10 \\
\hline 1 & 0.05 & 0.42 & 0.19 & 0.33 & 0.08 \\
\hline 1 & 0.1 & 0.47 & 0.15 & 0.39 & 0.16 \\
\hline 1 & 0.2 & 0.42 & 0.15 & 0.97 & 0.27 \\
\hline 1 & 0.5 & 0.48 & 0.24 & 0.26 & 0.04 \\
\hline 1 & 1 & 0.44 & 0.15 & 0.78 & 0.14 \\
\hline 1 & 5 & 0.11 & 0.08 & -0.05 & 0.05 \\
\hline 2 & 0 & 0.37 & 0.20 & 1.22 & 0.06 \\
\hline 2 & 0.05 & 0.42 & 0.06 & 1.11 & 0.04 \\
\hline 2 & 0.1 & 0.58 & 0.17 & 1.18 & 0.12 \\
\hline 2 & 0.2 & 0.46 & 0.14 & 1.14 & 0.08 \\
\hline 2 & 0.5 & 0.53 & 0.11 & 1.23 & 0.06 \\
\hline 2 & 1 & 0.37 & 0.24 & 1.20 & 0.09 \\
\hline 2 & 5 & 0.69 & 0.12 & 0.83 & 0.19 \\
\hline 4 & 0 & 0.62 & 0.16 & 0.97 & 0.10 \\
\hline 4 & 0.05 & 0.67 & 0.11 & 0.91 & 0.05 \\
\hline 4 & 0.1 & 0.67 & 0.09 & 0.73 & 0.12 \\
\hline 4 & 0.2 & 0.72 & 0.16 & 1.14 & 0.09 \\
\hline 4 & 0.5 & 0.59 & 0.03 & 1.08 & 0.12 \\
\hline 4 & 1 & 0.52 & 0.07 & 1.06 & 0.05 \\
\hline 4 & 5 & 0.60 & 0.07 & 0.24 & 0.07 \\
\hline 5 & 0 & 0.40 & 0.14 & 0.57 & 0.16 \\
\hline 5 & 0.05 & 0.24 & 0.22 & 0.60 & 0.13 \\
\hline 5 & 0.1 & 0.15 & 0.11 & 0.67 & 0.10 \\
\hline 5 & 0.2 & 0.38 & 0.10 & 0.57 & 0.12 \\
\hline 5 & 0.5 & 0.18 & 0.19 & 0.56 & 0.10 \\
\hline 5 & 1 & 0.34 & 0.06 & 0.71 & 0.07 \\
\hline 5 & 5 & 0.38 & 0.10 & 0.33 & 0.13 \\
\hline 6 & 0 & 0.37 & 0.10 & 1.09 & 0.06 \\
\hline 6 & 0.05 & 0.28 & 0.03 & 1.03 & 0.07 \\
\hline 6 & 0.1 & 0.33 & 0.14 & 0.87 & 0.21 \\
\hline 6 & 0.2 & 0.24 & 0.09 & 1.00 & 0.10 \\
\hline 6 & 0.5 & 0.25 & 0.20 & 0.99 & 0.14 \\
\hline 6 & 1 & 0.17 & 0.13 & 0.99 & 0.05 \\
\hline 6 & 5 & 0.18 & 0.08 & 0.46 & 0.07 \\
\hline
\end{tabular}




\begin{tabular}{|c|c|c|c|c|c|c|}
\hline 7 & & 0 & 0.63 & 0.20 & 1.28 & 0.16 \\
\hline 7 & & 0.05 & 0.56 & 0.13 & 1.32 & 0.09 \\
\hline 7 & & 0.075 & 0.51 & 0.06 & 1.33 & 0.12 \\
\hline 7 & & 0.1 & 0.52 & 0.06 & 1.33 & 0.12 \\
\hline 7 & & 0.15 & 0.54 & 0.12 & 1.16 & 0.01 \\
\hline 7 & & 0.2 & 0.54 & 0.12 & 1.16 & 0.01 \\
\hline 7 & & 0.5 & 0.35 & 0.10 & 1.40 & 0.19 \\
\hline \multicolumn{7}{|c|}{ PtNOA + decadienal } \\
\hline 1 & & 0 & 0.21 & 0.15 & 1.49 & 0.09 \\
\hline 1 & & 0.05 & 0.35 & 0.02 & 1.34 & 0.04 \\
\hline 1 & & 0.1 & 0.23 & 0.07 & 1.45 & 0.08 \\
\hline 1 & & 0.2 & 0.09 & 0.17 & 1.41 & 0.04 \\
\hline 1 & & 0.5 & 0.14 & 0.06 & 1.58 & 0.14 \\
\hline 1 & & 1 & 0.14 & 0.06 & 1.59 & 0.08 \\
\hline 1 & & 5 & 0.27 & 0.07 & 1.63 & 0.04 \\
\hline 2 & & 0 & 0.27 & 0.09 & 1.11 & 0.06 \\
\hline 2 & & 0.05 & 0.37 & 0.17 & 1.15 & 0.09 \\
\hline 2 & & 0.1 & 0.56 & 0.05 & 1.01 & 0.24 \\
\hline 2 & & 0.2 & 0.68 & 0.06 & 1.02 & 0.10 \\
\hline 2 & & 0.5 & 0.54 & 0.08 & 1.04 & 0.10 \\
\hline 2 & & 1 & 0.66 & 0.15 & 1.01 & 0.03 \\
\hline 2 & & 5 & 0.57 & 0.15 & 0.88 & 0.23 \\
\hline 3 & & 0 & 0.75 & 0.16 & 0.86 & 0.19 \\
\hline 3 & & 0.05 & 0.61 & 0.11 & -0.03 & 0.22 \\
\hline 3 & & 0.1 & 0.48 & 0.26 & 0.75 & 0.06 \\
\hline 3 & & 0.2 & 0.72 & 0.12 & 0.80 & 0.14 \\
\hline 3 & & 0.5 & 0.46 & 0.16 & 0.64 & 0.25 \\
\hline 3 & & 1 & 0.45 & 0.06 & 0.66 & 0.30 \\
\hline 3 & & 5 & 0.55 & 0.24 & 0.69 & 0.14 \\
\hline \multicolumn{7}{|c|}{ WT + 15(s)-HpETE } \\
\hline & 1 & 0 & 1.00 & 0.24 & 0.68 & 0.15 \\
\hline & 1 & 0.0005 & 0.71 & 0.14 & 0.77 & 0.14 \\
\hline & 1 & 0.001 & 0.78 & 0.11 & 0.56 & 0.10 \\
\hline & 1 & 0.005 & 0.61 & 0.10 & 0.61 & 0.07 \\
\hline & 1 & 0.01 & 0.64 & 0.09 & 0.39 & 0.16 \\
\hline & 1 & 0.05 & 0.51 & 0.12 & 0.79 & 0.14 \\
\hline & 1 & 0.1 & 0.46 & 0.08 & 0.46 & 0.15 \\
\hline & 1 & 0.5 & 0.46 & 0.19 & 0.14 & 0.20 \\
\hline & 2 & 0 & 0.78 & 0.09 & 1.03 & 0.15 \\
\hline & 2 & 0.0005 & 0.86 & 0.08 & 0.76 & 0.42 \\
\hline & 2 & 0.001 & 0.84 & 0.08 & 0.90 & 0.37 \\
\hline & 2 & 0.005 & 0.63 & 0.32 & 1.05 & 0.08 \\
\hline & 2 & 0.01 & 0.85 & 0.22 & 0.94 & 0.31 \\
\hline & 2 & 0.05 & 0.65 & 0.10 & 1.05 & 0.10 \\
\hline & 2 & 0.1 & 0.81 & 0.21 & 0.65 & 0.39 \\
\hline
\end{tabular}




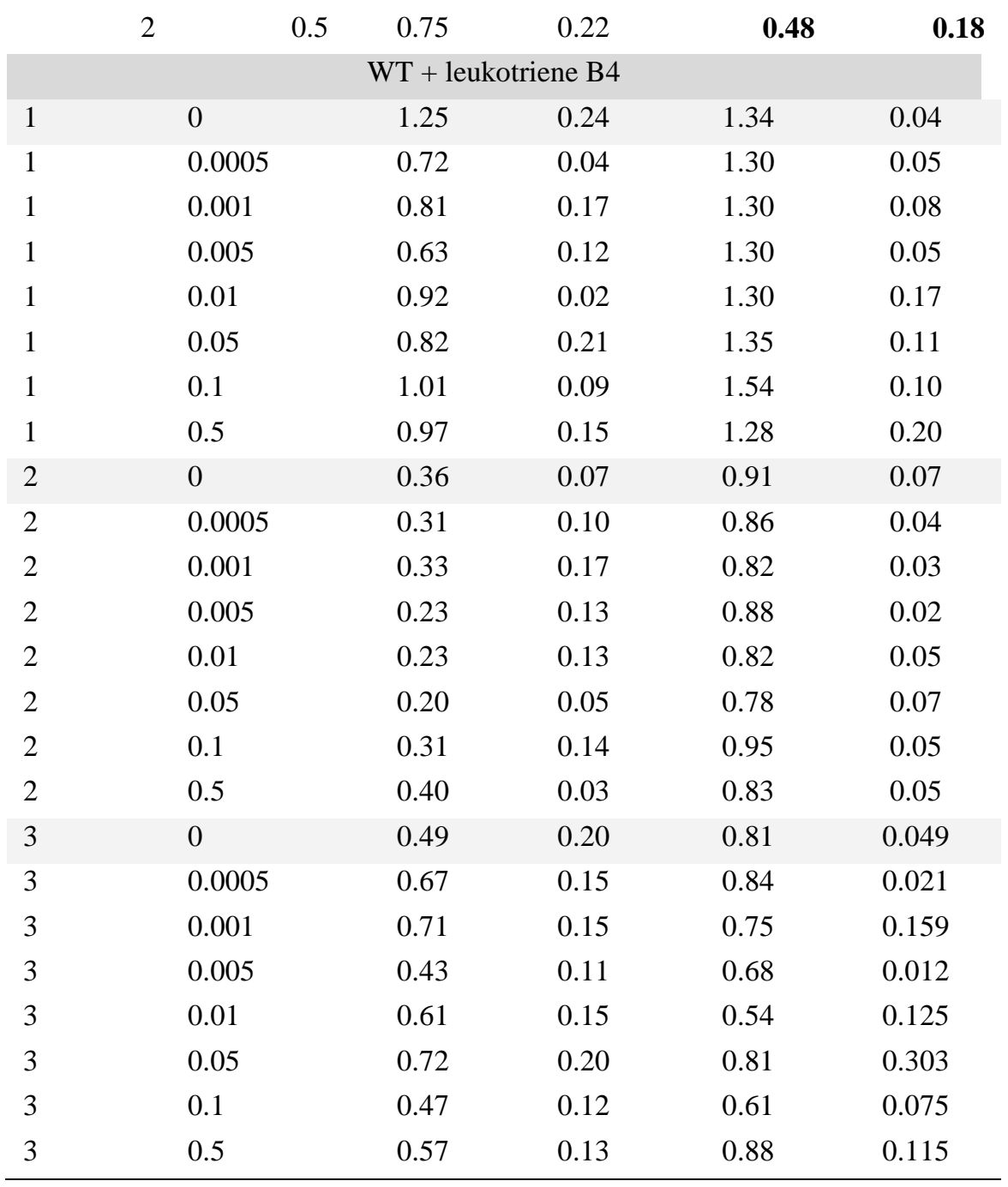


Table 2. Retention times (RT) of authentic standards. Abbreviations for oxylipins can be found in Appendix A.

\begin{tabular}{|c|c|c|}
\hline Standard & $\begin{array}{c}\text { Structural level } \\
\text { annotation }\end{array}$ & RT \\
\hline Octanoic acid & FFA 8:0 & 2.65 \\
\hline Arachidonic acid & FFA 20:4 & 4.02 \\
\hline Docasahexaenoic acid & FFA 22:6 & 7.71 \\
\hline 9-HpODE & NVO 18:2 20 & 5.44 \\
\hline 13-HpODE & NVO 18:2 20 & 5.44 \\
\hline 9-HOTrE & NVO 18:310 & 4.02 \\
\hline 13-HpOTrE & NVO 18:3 20 & 4.65 \\
\hline 9-HpOTrE & NVO 18:3 20 & 4.28 \\
\hline 9-KOTrE & NVO 18:4 10 & 4.10 \\
\hline 15-HETrE & NVO 20:3 10 & 6.11 \\
\hline 15-HETE & NVO 20:4 10 & 5.43 \\
\hline 5-HpETE & NVO 20:5 20 & 5.01 \\
\hline 12-HpETE & NVO 20:5 20 & 5.95 \\
\hline 15-HрETE & NVO 20:5 20 & 5.73 \\
\hline 5,6-diHETE & NVO 20:5 20 & 3.48 \\
\hline 14,15-diHETE & NVO 20:5 20 & 3.00 \\
\hline 15-HEPE & NVO 20:5 10 & 4.56 \\
\hline 15-KETE & NVO 20:5 10 & 5.24 \\
\hline 12-KETE & NVO 20:5 10 & 5.63 \\
\hline Heptadienal & PUA 7:2 & 1.90 \\
\hline Octadienal & PUA 8:2 & 2.28 \\
\hline Nondienal & PUA 9:2 & 2.69 \\
\hline Decadienal & PUA 10:2 & 3.65 \\
\hline
\end{tabular}


Table 3. Functional group level annotations of the features with structural level annotations matching the authentic standards. Retention times of authentic standards ( $\mathrm{RT}_{\text {auth}}$ ) presented in Table 2 were compared to the observed retention times of structural isomers (RTobs) in the dissolved lipidome. Putative functional group annotations were assigned to -10 $\mathrm{NVO}$, fatty acids, and PUAs features that had $\mathrm{RT}_{\text {obs }}$ within 0.5 minutes of the $\mathrm{RT}_{\text {auth }}$ and $-2 \mathrm{O} \mathrm{NVO}$ features that had a $\mathrm{RT}_{\mathrm{obs}}$ with 0.7 minutes of the $\mathrm{RT}_{\text {auth }}$. Abbreviations for oxylipins can be found in Appendix A.

\begin{tabular}{llllll}
\hline Structural annotation & $\mathrm{RT}_{\text {obs }}$ & Standard & $\mathrm{RT}_{\text {auth }}$ & $\mathrm{Rt}_{\text {obs-RT }}$ auth & $\begin{array}{l}\text { Proposed functional } \\
\text { group annotation }\end{array}$ \\
\hline FFA 20:4 & 8.57 & AA & 4.02 & 4.55 & \\
PUA 10:2 & 1.64 & Decadienal & 3.65 & -2.01 & \\
PUA 9:2 & 1.48 & Nonadienal & 2.69 & -1.21 & \\
NVO 18:3 1O RT-5.1 & 5.1 & 9-HOTrE & 5.02 & $\mathbf{0 . 0 8}$ & HOTrE (2b) \\
NVO 20:3 1O RT-6.62 & 6.62 & 15-HETrE & 6.11 & 0.51 & $\operatorname{HETrE~(2b)~}$ \\
NVO 20:4 2O RT-2.66 & 2.66 & 14,15-diHETE & 3 & $\mathbf{- 0 . 3 4}$ & diHETE (2b)
\end{tabular}


Table 4a. Functional group level annotations of features annotated as small fatty acids ( $<14$ carbons) within the dissolved lipidome. The retentions time of the octanoic acid authentic standard (RTauth) presented in Table 2 was used to predict retention times for structural

isomers (RTcalc). The difference in carbon number $(\mathrm{dC})$ and double bond number (dDB) was calculated for the structural annotation of the observed feature and the structural annotation of the authentic standard. Equation 1 was applied to determine the RT $\mathrm{T}_{\text {calc }}$. Putative functional group annotations were assigned to $-10 \mathrm{NVO}$, fatty acids, and PUAs features that had RTobs within 0.5 minutes of the RT $\mathrm{T}_{\text {calc }}$.

Abbreviations for oxylipins can be found in Appendix A.

\begin{tabular}{|c|c|c|c|c|c|c|c|c|c|}
\hline \multicolumn{2}{|l|}{ Observed feature } & \multicolumn{3}{|c|}{ Authentic Standard } & \multicolumn{4}{|c|}{ Calculation } & \multirow{2}{*}{$\begin{array}{l}\text { Proposed } \\
\text { Functional } \\
\text { Group } \\
\text { Annotation }\end{array}$} \\
\hline Structural Annotation & $\mathrm{RT}_{\mathrm{obs}}$ & $\begin{array}{l}\text { Structural } \\
\text { Annotation }\end{array}$ & Standard Name & $\mathrm{RT}_{\text {auth }}$ & $\mathrm{dC}$ & dDB & $\mathrm{RT}_{\text {calc }}$ & $\mathrm{RT}_{\text {obs }}-\mathrm{RT}_{\text {cal }}$ & \\
\hline FFA 10:0 none & 4.36 & FFA 8:0 & Octanoic acid & 2.65 & 2 & 0 & 4.75 & -0.39 & $\begin{array}{l}\text { Decanoic acid } \\
\text { (3b) }\end{array}$ \\
\hline FFA 12:2 none & 1.46 & FFA 8:0 & Octanoic acid & 2.65 & 4 & 2 & 5.05 & -3.59 & \\
\hline
\end{tabular}


Table 4b. Functional group level annotations of features annotated as small non-volatile oxylipins ( $<14$ carbons) within the dissolved lipidome. The retentions times of the $\mathrm{C} 18$ authentic standards $\left(\mathrm{RT}_{\text {auth }}\right)$ presented in Table 2 were used to predict retention times for structural isomers $\left(\mathrm{RT}_{\text {calc }}\right)$. The difference in carbon number $(\mathrm{dC})$ and double bond number (dDB) was calculated for the structural annotation of the observed feature and the structural annotation of the authentic standard. Equation 1 was applied to determine the $\mathrm{RT}_{\text {calc }}$. Putative functional group annotations were assigned to $-1 \mathrm{O} \mathrm{NVO}$ features that had $\mathrm{RT}_{\mathrm{obs}}$ within 0.5 minutes of the $\mathrm{RT}$ calc. and $-2 \mathrm{O} \mathrm{NVO}$ features that had $\mathrm{RT}_{\mathrm{obs}}$ within 0.7 minutes of the $\mathrm{RT}_{\text {calc. }}$. Abbreviations for oxylipins can be found in Appendix A.

\begin{tabular}{|c|c|c|c|c|c|c|c|c|c|}
\hline \multicolumn{2}{|l|}{ Observed Feature } & \multicolumn{5}{|l|}{ Authentic Standard } & \multicolumn{2}{|c|}{ Calculation } & \multirow{2}{*}{$\begin{array}{l}\text { Proposed Functional Group } \\
\text { Annotation }\end{array}$} \\
\hline $\begin{array}{l}\text { Structural } \\
\text { Annotation }\end{array}$ & $\mathrm{RT}_{\mathrm{obs}}$ & Structural Annotation & Standard Name & $\mathrm{RT}_{\text {auth }}$ & $\mathrm{dC}$ & $\mathrm{dDB}$ & $\mathrm{RT}_{\text {calc }}$ & $\mathrm{RT}_{\mathrm{obs}}-\mathrm{RT}_{\mathrm{cal}}$ & \\
\hline \multirow[t]{2}{*}{ NVO 10:0 10 } & 1.77 & NVO 18:310 & 9-HOTrE & 4.02 & -8 & -3 & -1.68 & 3.45 & \\
\hline & & NVO $18: 410$ & 9-KOTrE & 4.1 & -8 & -4 & -0.7 & 0.7 & \\
\hline \multirow[t]{3}{*}{ NVO 10:0 20} & 1.48 & NVO $18: 220$ & 9-HpODE & 5.44 & -8 & -2 & -1.16 & 2.64 & \\
\hline & & NVO $18: 320$ & 9-HpOTrE & 4.28 & -8 & -3 & -1.42 & 1.42 & \\
\hline & & NVO 18:3 20 & 13-HpOTrE & 4.65 & -8 & -3 & -1.05 & 1.05 & \\
\hline \multirow{3}{*}{ NVO 10:1 20} & 1.64 & NVO 18:2 20 & 9-HpODE & 5.44 & -8 & -1 & -2.06 & 3.7 & \\
\hline & & NVO $18: 320$ & 9-HpOTrE & 4.28 & -8 & -2 & -2.32 & 2.32 & \\
\hline & & NVO $18: 320$ & 13-HpOTrE & 4.65 & -8 & -2 & -1.95 & 1.95 & \\
\hline \multirow[t]{3}{*}{ NVO 12:0 20} & 1.7 & NVO $18: 220$ & 9-HpODE & 5.44 & -6 & -2 & 0.94 & 0.76 & \\
\hline & & NVO $18: 320$ & 9-HpOTrE & 4.28 & -6 & -3 & 0.68 & -0.68 & HpDA (3b) \\
\hline & & NVO $18: 320$ & 13-HpOTrE & 4.65 & -6 & -3 & 1.05 & -1.05 & \\
\hline \multirow[t]{3}{*}{ NVO 12:1 20} & 1.81 & NVO $18: 220$ & 9-HpODE & 5.44 & -6 & -1 & 0.04 & 1.77 & \\
\hline & & NVO $18: 320$ & 9-HpOTrE & 4.28 & -6 & -2 & -0.22 & 0.22 & HpDMA (3b) \\
\hline & & NVO $18: 320$ & 13-HpOTrE & 4.65 & -6 & -2 & 0.15 & -0.15 & \\
\hline \multirow[t]{3}{*}{ NVO 14:0 20} & 2 & NVO $18: 220$ & 9-HpODE & 5.44 & -4 & -2 & 3.04 & -1.04 & \\
\hline & & NVO $18: 320$ & 9-HpOTrE & 4.28 & -4 & -3 & 2.78 & -0.78 & \\
\hline & & NVO $18: 320$ & 13-HpOTrE & 4.65 & -4 & -3 & 3.15 & -1.15 & \\
\hline \multirow{3}{*}{ NVO 14:0 20} & 2.94 & NVO $18: 220$ & 9-HpODE & 5.44 & -4 & -2 & 3.04 & -0.1 & HpTA (3b) \\
\hline & & NVO $18: 320$ & 9-HpOTrE & 4.28 & -4 & -3 & 2.78 & 0.16 & \\
\hline & & NVO $18: 320$ & 13-HpOTrE & 4.65 & -4 & -3 & 3.15 & -0.21 & \\
\hline \multirow[t]{3}{*}{ NVO 14:1 20} & 4.16 & NVO $18: 220$ & 9-HpODE & 5.44 & -4 & -1 & 2.14 & 2.02 & \\
\hline & & NVO $18: 320$ & 9-HpOTrE & 4.28 & -4 & -2 & 1.88 & 2.28 & \\
\hline & & NVO $18: 320$ & 13-HpOTrE & 4.65 & -4 & -2 & 2.25 & 1.91 & \\
\hline \multirow[t]{2}{*}{ NVO 14:2 10} & 2.42 & NVO $18: 310$ & 9-HOTrE & 4.02 & -4 & -1 & 0.72 & 1.7 & \\
\hline & & NVO $18: 410$ & 9-KOTrE & 4.1 & -4 & -2 & 1.7 & 0.72 & \\
\hline \multirow[t]{2}{*}{ NVO 14:2 10} & 4.38 & NVO $18: 310$ & 9-HOTrE & 4.02 & -4 & -1 & 0.72 & 3.66 & \\
\hline & & NVO $18: 410$ & 9-KOTrE & 4.1 & -4 & -2 & 1.7 & 2.68 & \\
\hline \multirow[t]{3}{*}{ NVO 14:2 20} & 2.26 & NVO $18: 220$ & 9-HpODE & 5.44 & -4 & 0 & 1.24 & 1.02 & \\
\hline & & NVO $18: 320$ & 9-HpOTrE & 4.28 & -4 & -1 & 0.98 & 1.28 & \\
\hline & & NVO $18: 320$ & 13-HpOTrE & 4.65 & -4 & -1 & 1.35 & 0.91 & \\
\hline
\end{tabular}


Table 4c. Functional group level annotations of features annotated as C16 non-volatile oxylipins within the dissolved lipidome. The retentions times of the C18 authentic standards $\left(\mathrm{RT}_{\text {auth }}\right)$ presented in Table 2 were used to predict retention times for structural isomers $\left(\mathrm{RT}_{\text {calc }}\right)$. The difference in carbon number $(\mathrm{dC})$ and double bond number (dDB) was calculated for the structural annotation of the observed feature and the structural annotation of the authentic standard. Equation 1 was applied to determine the $\mathrm{RT}_{\text {calc }}$. Putative functional group annotations were assigned to $-1 \mathrm{O} N V O$ features that had $\mathrm{RT}_{\text {obs }}$ within 0.5 minutes of the $\mathrm{RT}_{\text {calc }}$. and $-2 \mathrm{O} \mathrm{NVO}$ features that had $\mathrm{RT}_{\mathrm{obs}}$ within 0.7 minutes of the $\mathrm{RT}_{\text {calc }}$. Abbreviations for oxylipins can be found in Appendix $\mathrm{A}$

\begin{tabular}{|c|c|c|c|c|c|c|c|c|c|}
\hline \multicolumn{2}{|c|}{ Observed Feature } & \multicolumn{3}{|c|}{ Authentic Standard } & \multicolumn{4}{|c|}{ Calculation } & \multirow[b]{2}{*}{ Proposed Functional Group Annotation } \\
\hline $\begin{array}{l}\text { Structural } \\
\text { Annotation }\end{array}$ & $\mathrm{RT}_{\mathrm{obs}}$ & Structural Annotation & Standard Names & $\mathrm{RT}_{\text {auth }}$ & $\mathrm{dC}$ & $\mathrm{dDB}$ & $\mathrm{RT}_{\text {calc }}$ & $\mathrm{RT}_{\mathrm{obs}}-\mathrm{RT}_{\mathrm{cal}}$ & \\
\hline \multirow[t]{2}{*}{ NVO 16:0 10} & 5 & NVO 18:310 & 9-HOTrE & 4.02 & -2 & -3 & 4.62 & 0.38 & HНA (3b) \\
\hline & & NVO $18: 410$ & 9-KOTrE & 4.1 & -2 & -4 & 5.6 & -0.6 & \\
\hline \multirow[t]{3}{*}{ NVO 16:0 20} & 2.47 & NVO $18: 220$ & 9-HpODE & 5.44 & -2 & -2 & 5.14 & -2.67 & \\
\hline & & NVO 18:32O & 9-HpOTrE & 4.28 & -2 & -3 & 4.88 & -2.41 & \\
\hline & & NVO 18:32O & 13-HpOTrE & 4.65 & -2 & -3 & 5.25 & -2.78 & \\
\hline \multirow[t]{3}{*}{ NVO 16:0 20} & 4.84 & NVO 18:2 20 & 9-HpODE & 5.44 & -2 & -2 & 5.14 & -0.3 & HрHA (3b) \\
\hline & & NVO $18: 320$ & 9-HpOTrE & 4.28 & -2 & -3 & 4.88 & -0.04 & \\
\hline & & NVO 18:3 20 & 13-HpOTrE & 4.65 & -2 & -3 & 5.25 & -0.41 & \\
\hline \multirow[t]{2}{*}{ NVO 16:1 10} & 3.79 & NVO 18:310 & 9-HOTrE & 4.02 & -2 & -2 & 3.72 & 0.07 & HHME (3b) \\
\hline & & NVO 18:4 10 & 9-KOTrE & 4.1 & -2 & -3 & 4.7 & -0.91 & \\
\hline \multirow[t]{3}{*}{ NVO 16:1 20} & 2.25 & NVO 18:2 20 & 9-HpODE & 5.44 & -2 & -1 & 4.24 & -1.99 & \\
\hline & & NVO 18:3 20 & 9-HpOTrE & 4.28 & -2 & -2 & 3.98 & -1.73 & \\
\hline & & NVO 18:32O & 13-HpOTrE & 4.65 & -2 & -2 & 4.35 & -2.1 & \\
\hline \multirow[t]{2}{*}{ NVO 16:2 10} & 4.78 & NVO 18:310 & 9-HOTrE & 4.02 & -2 & -1 & 2.82 & 1.96 & \\
\hline & & NVO $18: 410$ & 9-KOTrE & 4.1 & -2 & -2 & 3.8 & 0.98 & \\
\hline \multirow[t]{3}{*}{ NVO 16:2 20} & 2.69 & NVO 18:2 20 & 9-HpODE & 5.44 & -2 & 0 & 3.34 & -0.65 & HpHDE (3b) \\
\hline & & NVO 18:32O & 9-HpOTrE & 4.28 & -2 & -1 & 3.08 & -0.39 & \\
\hline & & NVO 18:32O & 13-HpOTrE & 4.65 & -2 & -1 & 3.45 & -0.76 & \\
\hline \multirow[t]{2}{*}{ NVO $16: 310$} & 2.78 & NVO $18: 310$ & 9-HOTrE & 4.02 & -2 & 0 & 1.92 & 0.86 & \\
\hline & & NVO 18:4 10 & 9-KOTrE & 4.1 & -2 & -1 & 2.9 & -0.12 & KHDE (3b) \\
\hline \multirow[t]{2}{*}{ NVO 16:3 10 } & 4.33 & NVO 18:310 & 9-HOTrE & 5.02 & -2 & 0 & 2.92 & 1.41 & \\
\hline & & NVO 18:4 10 & 9-KOTrE & 4.1 & -2 & -1 & 2.9 & 1.43 & \\
\hline \multirow[t]{3}{*}{ NVO 16:3 20} & 2.32 & NVO 18:3 20 & 9-HpOTrE & 4.28 & -2 & 0 & 2.18 & 0.14 & HpHTrE (3b) \\
\hline & & NVO $18: 320$ & 13-HpOTrE & 4.65 & -2 & 0 & 2.55 & -0.23 & \\
\hline & & NVO 18:2 20 & 9-HpODE & 5.44 & -2 & 1 & 2.44 & -0.12 & \\
\hline NVO 16:4 10 & 1.91 & NVO 18:4 10 & 9-KOTrE & 4.1 & -2 & 0 & 2 & -0.09 & KHTrE/HHTE (3b) \\
\hline
\end{tabular}




\begin{tabular}{llllrrrrrr} 
& & NVO 18:3 1O & 9-HOTrE & 5.02 & -2 & 1 & 2.02 & $\mathbf{- 0 . 1 1}$ \\
NVO 16:4 1O & 2.81 & NVO 18:4 1O & 9-KOTrE & 4.1 & -2 & 0 & 2 & 0.81 & \\
& & NVO 18:3 1O & 9-HOTrE & 5.02 & -2 & 1 & 2.02 & 0.79 & \\
\multirow{2}{*}{ NVO 16:4 2O } & 2.09 & NVO 18:3 2O & 9-HpOTrE & 4.28 & -2 & 1 & 1.28 & 0.81 & \\
& & NVO 18:3 2O & 13-HpOTrE & 4.65 & -2 & 1 & 1.65 & $\mathbf{0 . 4 4}$ & HpHTE (3b) \\
& & NVO 18:2 2O & 9-HpODE & 5.44 & -2 & 2 & 1.54 & $\mathbf{0 . 5 5}$ &
\end{tabular}


Table 4d. Functional group level annotations of features annotated as C18 non-volatile oxylipins within the dissolved lipidome. The retentions times of the C18 authentic standards $\left(\mathrm{RT}_{\text {auth }}\right)$ presented in Table 2 were used to predict retention times for structural isomers $\left(\mathrm{RT}_{\text {cac }}\right)$. The difference in carbon number $(\mathrm{dC})$ and double bond number ( $\mathrm{dDB}$ ) was calculated for the structural annotation of the observed feature and the structural annotation of the authentic standard. Equation 1 was applied to determine the $\mathrm{RT}_{\text {calc }}$. Putative functional group annotations were assigned to $-1 \mathrm{O} \mathrm{NVO}$ features that had $\mathrm{RT}_{\text {obs }}$ within 0.5 minutes of the RT $\mathrm{T}_{\text {calc }}$. and $2 \mathrm{O} N V O$ features that had $\mathrm{RT}_{\mathrm{obs}}$ within 0.7 minutes of the $\mathrm{RT}_{\text {calc. }}$. Abbreviations for oxylipins can be found in Appendix A.

\begin{tabular}{|c|c|c|c|c|c|c|c|c|c|}
\hline \multicolumn{2}{|c|}{ Observed feature } & \multicolumn{3}{|c|}{ Authentic Standard } & \multicolumn{4}{|c|}{ Calculation } & \multirow[b]{2}{*}{ Proposed Functional Group annotation } \\
\hline $\begin{array}{l}\text { Structural } \\
\text { Annotation }\end{array}$ & $\mathrm{RT}_{\mathrm{obs}}$ & Structural Annotation & Standard Name & $\mathrm{RT}_{\text {auth }}$ & $\mathrm{dC}$ & dDB & $\mathrm{RT}_{\text {calc }}$ & $\mathrm{RT}_{\mathrm{obs}}-\mathrm{RT}_{\mathrm{cal}}$ & \\
\hline \multirow[t]{2}{*}{ NVO 18:0 10} & 6.77 & NVO 18:4 10 & 9-KOTrE & 4.1 & 0 & -4 & 7.7 & -0.93 & \\
\hline & & NVO $18: 310$ & 9-HOTrE & 5.02 & 0 & -3 & 7.72 & -0.95 & \\
\hline \multirow[t]{3}{*}{ NVO 18:0 20} & 3.01 & NVO 18:3 20 & 9-HpOTrE & 4.28 & 0 & -3 & 6.98 & -3.97 & \\
\hline & & NVO 18:3 20 & 13-HpOTrE & 4.65 & 0 & -3 & 7.35 & -4.34 & \\
\hline & & NVO 18:2 20 & 9-HpODE & 5.44 & 0 & -2 & 7.24 & -4.23 & \\
\hline \multirow[t]{3}{*}{ NVO 18:0 20} & 4.41 & NVO 18:3 20 & 9-HpOTrE & 4.28 & 0 & -3 & 6.98 & -2.57 & \\
\hline & & NVO 18:3 20 & 13-HpOTrE & 4.65 & 0 & -3 & 7.35 & -2.94 & \\
\hline & & NVO 18:2 20 & 9-HpODE & 5.44 & 0 & -2 & 7.24 & -2.83 & \\
\hline \multirow[t]{2}{*}{ NVO 18:1 10} & 5.75 & NVO 18:4 10 & 9-KOTrE & 4.1 & 0 & -3 & 6.8 & -1.05 & \\
\hline & & NVO 18:3 10 & 9-HOTrE & 5.02 & 0 & -2 & 6.82 & -1.07 & \\
\hline \multirow[t]{3}{*}{ NVO 18:1 20} & 3.11 & NVO 18:3 20 & 9-HpOTrE & 4.28 & 0 & -2 & 6.08 & -2.97 & \\
\hline & & NVO 18:3 20 & 13-HpOTrE & 4.65 & 0 & -2 & 6.45 & -3.34 & \\
\hline & & NVO 18:2 20 & 9-HpODE & 5.44 & 0 & -1 & 6.34 & -3.23 & \\
\hline \multirow[t]{2}{*}{ NVO 18:2 10} & 6.26 & NVO 18:4 10 & 9-KOTrE & 4.1 & 0 & -2 & 5.9 & 0.36 & KOME/HODE (3b) \\
\hline & & NVO 18:3 10 & 9-HOTrE & 5.02 & 0 & -1 & 5.92 & 0.34 & \\
\hline \multirow[t]{2}{*}{ NVO 18:3 10} & 5.1 & NVO 18:4 10 & 9-KOTrE & 4.1 & 0 & -1 & 5 & 0.1 & KODE/HOTrE \\
\hline & & NVO 18:3 10 & 9-HOTrE & 5.02 & 0 & 0 & 5.02 & 0.08 & HOTrE (2b) \\
\hline \multirow[t]{2}{*}{ NVO 18:4 10} & 2.59 & NVO 18:4 10 & 9-KOTrE & 4.1 & 0 & 0 & 4.1 & -1.51 & \\
\hline & & NVO $18: 310$ & 9-HOTrE & 5.02 & 0 & 1 & 4.12 & -1.53 & \\
\hline \multirow[t]{2}{*}{ NVO 18:5 10} & 2.44 & NVO 18:4 10 & 9-KOTrE & 4.1 & 0 & 1 & 3.2 & -0.76 & \\
\hline & & NVO $18: 310$ & 9-HOTrE & 5.02 & 0 & 2 & 3.22 & -0.78 & \\
\hline \multirow[t]{2}{*}{ NVO $18: 510$} & 4.29 & NVO $18: 410$ & 9-KOTrE & 4.1 & 0 & 1 & 3.2 & 1.09 & \\
\hline & & NVO $18: 310$ & 9-HOTrE & 5.02 & 0 & 2 & 3.22 & 1.07 & \\
\hline \multirow[t]{3}{*}{ NVO 18:5 20} & 4.32 & NVO 18:3 20 & 9-HpOTrE & 4.28 & 0 & 2 & 2.48 & 1.84 & \\
\hline & & NVO 18:3 20 & 13-HpOTrE & 4.65 & 0 & 2 & 2.85 & 1.47 & \\
\hline & & NVO 18:2 20 & 9-HpODE & 5.44 & 0 & 3 & 2.74 & 1.58 & \\
\hline
\end{tabular}


Table 4e. Functional group level annotations of features annotated as C20:3 non-volatile oxylipins within the dissolved lipidome. The retentions times of the C20 authentic standards $\left(\mathrm{RT}_{\text {auth }}\right)$ presented in Table 2 were used to predict retention times for structural isomers $\left(\mathrm{RT}_{\text {calc }}\right)$. The difference in carbon number $(\mathrm{dC})$ and double bond number (dDB) was calculated for the structural annotation of the observed feature and the structural annotation of the authentic standard. Equation 1 was applied to determine the $\mathrm{RT}_{\text {calc }}$. Putative functional group annotations were assigned to $-1 \mathrm{O} \mathrm{NVO}$ features that had $\mathrm{RT}_{\text {obs }}$ within 0.5 minutes of the RT $\mathrm{T}_{\text {calc }}$. and $-2 \mathrm{O} \mathrm{NVO}$ features that had $\mathrm{RT}_{\mathrm{obs}}$ within 0.7 minutes of the $\mathrm{RT}_{\text {calc }}$. Abbreviations for oxylipins can be found in Appendix A.

\begin{tabular}{|c|c|c|c|c|c|c|c|c|c|}
\hline \multicolumn{2}{|c|}{ Observed feature } & \multicolumn{3}{|c|}{ Authentic Standard } & \multicolumn{4}{|c|}{ Calculation } & \multirow[b]{2}{*}{ Proposed Functional Group annotation } \\
\hline $\begin{array}{l}\text { Structural } \\
\text { Annotation }\end{array}$ & $\mathrm{RT}_{\mathrm{obs}}$ & Structural Annotation & Standard & $\mathrm{RT}_{\text {auth }}$ & $\mathrm{dC}$ & $\mathrm{dDB}$ & $\mathrm{RT}_{\text {calc }}$ & $\mathrm{RT}_{\mathrm{obs}}-\mathrm{RT}_{\mathrm{cal}}$ & \\
\hline \multirow[t]{5}{*}{ NVO 20:0 20} & 10.08 & NVO 20:4 20 & 5-HpETE & 5.01 & 0 & -4 & 8.61 & 1.47 & \\
\hline & & NVO 20:4 20 & 12-НpETE & 5.95 & 0 & -4 & 9.55 & $\mathbf{0 . 5 3}$ & HpEA (3b) \\
\hline & & NVO 20:4 20 & 15-HpETE & 5.73 & 0 & -4 & 9.33 & 0.75 & \\
\hline & & NVO 20:4 20 & 5,6 diHETE & 3.48 & 0 & -4 & 7.08 & 3 & \\
\hline & & NVO 20:4 20 & 14,15-diHETE & 3 & 0 & -4 & 6.6 & 3.48 & \\
\hline \multirow[t]{5}{*}{ NVO 20:1 10} & 7.38 & NVO 20:5 10 & 15-HEPE & 4.56 & 0 & -4 & 8.16 & -0.78 & \\
\hline & & NVO 20:5 10 & 15-KETE & 5.24 & 0 & -4 & 8.84 & -1.46 & \\
\hline & & NVO 20:5 10 & 12-KETE & 5.63 & 0 & -4 & 9.23 & -1.85 & \\
\hline & & NVO 20:4 10 & 15-HETE & 5.43 & 0 & -3 & 8.13 & -0.75 & \\
\hline & & NVO 20:3 10 & 15-HETrE & 6.11 & 0 & -2 & 7.91 & -0.53 & \\
\hline \multirow[t]{5}{*}{ NVO 20:1 20} & 6.77 & NVO 20:4 20 & 5-HpETE & 5.01 & 0 & -3 & 7.71 & -0.94 & \\
\hline & & NVO 20:4 20 & 12-HpETE & 5.95 & 0 & -3 & 8.65 & -1.88 & \\
\hline & & NVO 20:4 20 & 15-НpETE & 5.73 & 0 & -3 & 8.43 & -1.66 & \\
\hline & & NVO 20:4 20 & 5,6 diHETE & 3.48 & 0 & -3 & 6.18 & 0.59 & diEME (3b) \\
\hline & & NVO 20:4 20 & 14,15-diHETE & 3 & 0 & -3 & 5.7 & 1.07 & \\
\hline \multirow[t]{5}{*}{ NVO 20:3 10} & 6.62 & NVO 20:5 10 & 15-HEPE & 4.56 & 0 & -2 & 6.36 & 0.26 & HETrE/KETE \\
\hline & & NVO 20:5 10 & 15-KETE & 5.24 & 0 & -2 & 7.04 & -0.42 & \\
\hline & & NVO 20:5 10 & 12-KETE & 5.63 & 0 & -2 & 7.43 & -0.81 & \\
\hline & & NVO 20:4 10 & 15-HETE & 5.43 & 0 & -1 & 6.33 & 0.29 & HETrE (2b) \\
\hline & & NVO 20:3 10 & 15-HETrE & 6.11 & 0 & 0 & 6.11 & 0.51 & \\
\hline \multirow[t]{5}{*}{ NVO 20:3 20} & 2.22 & NVO 20:4 20 & 5-HpETE & 5.01 & 0 & -1 & 5.91 & -3.69 & \\
\hline & & NVO 20:4 20 & 12-HpETE & 5.95 & 0 & -1 & 6.85 & -4.63 & \\
\hline & & NVO 20:4 20 & 15-НpЕTE & 5.73 & 0 & -1 & 6.63 & -4.41 & \\
\hline & & NVO 20:4 20 & 5,6 diHETE & 3.48 & 0 & -1 & 4.38 & -2.16 & \\
\hline & & NVO 20:4 20 & 14,15-diHETE & 3 & 0 & -1 & 3.9 & -1.68 & \\
\hline
\end{tabular}


Table 4f. Functional group level annotations of features annotated as C20: 4 and C20:5 non-volatile oxylipins within the dissolved lipidome. The retentions times of the $\mathrm{C} 20$ authentic standards $\left(\mathrm{RT}_{\text {auth }}\right)$ presented in Table 2 were used to predict retention times for structural isomers $\left(\mathrm{RT}_{\text {calc }}\right)$. The difference in carbon number $(\mathrm{dC})$ and double bond number (dDB) was calculated for the structural annotation of the observed feature and the structural annotation of the authentic standard. Equation 1 was applied to determine the $\mathrm{RT}_{\text {calc }}$. Putative functional group annotations were assigned to $-1 \mathrm{O} \mathrm{NVO}$ features that had $\mathrm{RT}_{\text {obs }}$ within 0.5 minutes of the RT $\mathrm{T}_{\text {calc }}$. and $-2 \mathrm{O} \mathrm{NVO}$ features that had $\mathrm{RT}_{\mathrm{obs}}$ within 0.7 minutes of the $\mathrm{RT}_{\text {calc }}$. Abbreviations for oxylipins can be found in Appendix A.

\begin{tabular}{|c|c|c|c|c|c|c|c|c|c|}
\hline \multicolumn{2}{|c|}{ Observed feature } & \multicolumn{3}{|c|}{ Authentic Standard } & \multicolumn{4}{|c|}{ Calculation } & \multirow{2}{*}{$\begin{array}{l}\text { Proposed Functional Group } \\
\text { annotation }\end{array}$} \\
\hline Structural Annotation & $\mathrm{RT}_{\mathrm{obs}}$ & Structural Annotation & Standard & $\mathrm{RT}_{\text {auth }}$ & $\mathrm{dC}$ & $\mathrm{dDB}$ & $\mathrm{RT}_{\text {calc }}$ & $\mathrm{RT}_{\mathrm{obs}}-\mathrm{RT}_{\mathrm{cal}}$ & \\
\hline \multirow[t]{5}{*}{ NVO 20:4 20} & 2.66 & NVO 20:4 20 & 5-НpETE & 5.01 & 0 & 0 & 5.01 & -5.01 & \\
\hline & & NVO 20:4 20 & 12-HрETE & 5.95 & 0 & 0 & 5.95 & -3.29 & \\
\hline & & NVO 20:4 20 & 15-НpETE & 5.73 & 0 & 0 & 5.73 & -3.07 & \\
\hline & & NVO 20:4 20 & 5,6 diHETE & 3.48 & 0 & 0 & 3.48 & -0.82 & \\
\hline & & NVO 20:4 20 & 14,15-diHETE & 3 & 0 & 0 & 3 & -0.34 & diHETE (2b) \\
\hline \multirow[t]{5}{*}{ NVO 20:5 10} & 3.4 & NVO 20:5 10 & 15-HEPE & 4.56 & 0 & 0 & 4.56 & -1.16 & \\
\hline & & NVO 20:5 10 & 15-KETE & 5.24 & 0 & 0 & 5.24 & -1.84 & \\
\hline & & NVO 20:5 10 & 12-KETE & 5.63 & 0 & 0 & 5.63 & -2.23 & \\
\hline & & NVO 20:4 10 & 15-HETE & 5.43 & 0 & 1 & 4.53 & -1.13 & \\
\hline & & NVO 20:3 10 & 15-HETrE & 6.11 & 0 & 2 & 4.31 & -0.91 & \\
\hline \multirow[t]{5}{*}{ NVO 20:5 20} & 2.6 & NVO 20:4 20 & 5-HрETE & 5.01 & 0 & 1 & 4.11 & -1.51 & \\
\hline & & NVO 20:4 20 & 12-НpETE & 5.95 & 0 & 1 & 5.05 & -2.45 & \\
\hline & & NVO 20:4 20 & 15-НpETE & 5.73 & 0 & 1 & 4.83 & -2.23 & \\
\hline & & NVO 20:4 20 & 5,6 diHETE & 3.48 & 0 & 1 & 2.58 & 0.02 & diHEPE (3a) \\
\hline & & NVO 20:4 20 & 14,15-diHETE & 3 & 0 & 1 & 2.1 & 0.5 & \\
\hline
\end{tabular}


Table 4g. Functional group level annotations of features annotated as C22 non-volatile oxylipins within the dissolved lipidome. The retentions times of the C20 authentic standards $\left(\mathrm{RT}_{\text {auth }}\right)$ presented in Table 2 were used to predict retention times for structural isomers $\left(\mathrm{RT}_{\text {calc }}\right)$. The difference in carbon number $(\mathrm{dC})$ and double bond number $(\mathrm{dDB})$ was calculated for the structural annotation of the observed feature and the structural annotation of the authentic standard. Equation 1 was applied to determine the $\mathrm{RT}_{\text {calc }}$. Putative functional group annotations were assigned to $-1 \mathrm{O}$ NVO features that had $\mathrm{RT}_{\text {obs }}$ within 0.5 minutes of the $\mathrm{RT}_{\text {calc }}$. and $-2 \mathrm{O} \mathrm{NVO}$ features that had $\mathrm{RT}_{\mathrm{obs}}$ within 0.7 minutes of the $\mathrm{RT}_{\text {calc. }}$. Abbreviations for oxylipins can be found in Appendix A.

\begin{tabular}{|c|c|c|c|c|c|c|c|c|c|}
\hline \multicolumn{2}{|c|}{ Observed feature } & \multicolumn{3}{|c|}{ Authentic Standard } & \multicolumn{4}{|c|}{ Calculation } & \multirow[b]{2}{*}{ Proposed Functional Group Annotation } \\
\hline $\begin{array}{l}\text { Structural } \\
\text { Annotation }\end{array}$ & $\mathrm{RT}_{\text {obs }}$ & Structural Annotation & Standard Name & $\mathrm{RT}_{\text {auth }}$ & $\mathrm{dC}$ & $\mathrm{dDB}$ & $\mathrm{RT}_{\text {calc }}$ & $\mathrm{RT}_{\mathrm{obs}}-\mathrm{RT}_{\mathrm{cal}}$ & \\
\hline \multirow[t]{5}{*}{ NVO 22:1 20} & 7.73 & NVO 20:4 20 & 5-HpETE & 5.01 & 2 & -3 & 9.81 & -2.08 & \\
\hline & & NVO 20:4 20 & 12-НpETE & 5.95 & 2 & -3 & 10.75 & -3.02 & \\
\hline & & NVO 20:4 20 & 15-HpETE & 5.73 & 2 & -3 & 10.53 & -2.8 & \\
\hline & & NVO 20:4 20 & 5,6 diHETE & 3.48 & 2 & -3 & 8.28 & -0.55 & diDoME (3b) \\
\hline & & NVO 20:4 20 & 14,15-diHETE & 3 & 2 & -3 & 7.8 & -0.07 & \\
\hline \multirow[t]{5}{*}{ NVO $22: 220$} & 6.75 & NVO 20:4 20 & 5-HpETE & 5.01 & 2 & -2 & 8.91 & -2.16 & \\
\hline & & NVO 20:4 20 & 12-НpETE & 5.95 & 2 & -2 & 9.85 & -3.1 & \\
\hline & & NVO 20:4 20 & 15-НpETE & 5.73 & 2 & -2 & 9.63 & -2.88 & \\
\hline & & NVO 20:4 20 & 5,6 diHETE & 3.48 & 2 & -2 & 7.38 & -0.63 & $\operatorname{diDoDE}(3 b)$ \\
\hline & & NVO 20:4 20 & 14,15-diHETE & 3 & 2 & -2 & 6.9 & -0.15 & \\
\hline \multirow[t]{5}{*}{ NVO 22:4 20} & 8.59 & NVO 20:4 20 & 5-HpETE & 5.01 & 2 & 0 & 7.11 & 1.48 & \\
\hline & & NVO 20:4 20 & 12-НpETE & 5.95 & 2 & 0 & 8.05 & 0.54 & HpDoTE (3b) \\
\hline & & NVO 20:4 20 & 15-НpETE & 5.73 & 2 & 0 & 7.83 & 0.76 & \\
\hline & & NVO 20:4 20 & 5,6 diHETE & 3.48 & 2 & 0 & 5.58 & 3.01 & \\
\hline & & NVO 20:4 20 & 14,15-diHETE & 3 & 2 & 0 & 5.1 & 3.49 & \\
\hline \multirow[t]{5}{*}{ NVO 22:6 20} & 6.76 & NVO 20:4 20 & 5-HpETE & 5.01 & 2 & 2 & 5.31 & 1.45 & \\
\hline & & NVO 20:4 20 & 12-НpETE & 5.95 & 2 & 2 & 6.25 & 0.51 & HpDoHE (3b) \\
\hline & & NVO 20:4 20 & 15-НpETE & 5.73 & 2 & 2 & 6.03 & 0.73 & \\
\hline & & NVO 20:4 20 & 5,6 diHETE & 3.48 & 2 & 2 & 3.78 & 2.98 & \\
\hline & & NVO 20:4 20 & 14,15-diHETE & 3 & 2 & 2 & 3.3 & 3.46 & \\
\hline
\end{tabular}


Table 4h. Functional group level annotations of features annotated as PUAs within the dissolved lipidome. The retentions times of the PUA authentic standards $\left(\mathrm{RT}_{\text {auth }}\right)$ presented in Table 2 were used to predict retention times for structural isomers $\left(\mathrm{RT}_{\text {calc }}\right)$. The difference in carbon number ( $\mathrm{dC}$ ) and double bond number (dDB) was calculated for the structural annotation of the observed feature and the structural annotation of the authentic standard. Equation 1 was applied to determine the $\mathrm{RT}_{\text {calc }}$. Putative functional group annotations were assigned to features that had $\mathrm{RT}_{\text {obs }}$ within 0.5 minutes of the $\mathrm{RT}_{\text {calc. }}$ Elemental formula annotations were assigned to all other features.

\begin{tabular}{|c|c|c|c|c|c|c|c|c|c|c|}
\hline \multicolumn{2}{|c|}{ Observed feature } & \multicolumn{3}{|c|}{ Authentic Standard } & \multicolumn{4}{|c|}{ Calculation } & \multirow{2}{*}{$\begin{array}{c}\text { Proposed } \\
\text { Functional Group } \\
\text { Annotation }\end{array}$} & \multirow[b]{2}{*}{$\begin{array}{l}\text { Elemental Formula } \\
\text { Annotation }\end{array}$} \\
\hline Structural annotation & $\mathrm{RT}_{\mathrm{obs}}$ & $\begin{array}{l}\text { Structural } \\
\text { annotation }\end{array}$ & Standard & $\mathrm{RT}_{\text {auth }}$ & $\mathrm{dC}$ & $\mathrm{dDB}$ & $\mathrm{RT}_{\text {calc }}$ & $\mathrm{RT}_{\mathrm{obs}}-\mathrm{RT}_{\text {calc }}$ & & \\
\hline PUA 7:1 none & 1.59 & PUA 7:2 & heptadienal & 1.9 & 0 & -1 & 2.8 & -1.21 & & $\mathrm{C}_{7} \mathrm{H}_{12} \mathrm{O}$ \\
\hline PUA 8:3 none & 1.4 & PUA 8:2 & octadienal & 2.28 & 0 & 1 & 1.38 & 0.02 & octatrienal (3a) & \\
\hline PUA 9:0 none & 1.9 & PUA 9:2 & nonadienal & 2.69 & 0 & -2 & 4.49 & -2.59 & & $\mathrm{C}_{9} \mathrm{H}_{18} \mathrm{O}$ \\
\hline PUA 9:0 none & 3.45 & PUA 9:2 & nonadienal & 2.69 & 0 & -2 & 4.49 & -1.04 & & \\
\hline PUA 9:1 none & 1.8 & PUA 9:2 & nonadienal & 2.69 & 0 & -1 & 3.59 & -1.79 & & $\mathrm{C}_{9} \mathrm{H}_{16} \mathrm{O}$ \\
\hline PUA 9:3 none & 1.42 & PUA 9:2 & nonadienal & 2.69 & 0 & 1 & 1.79 & -0.37 & nonatrienal (3b) & \\
\hline PUA 10:0 none & 2.06 & PUA 10:2 & decadienal & 3.65 & 0 & -2 & 5.45 & -3.39 & & $\mathrm{C}_{10} \mathrm{H}_{20} \mathrm{O}$ \\
\hline PUA 10:1 none & 1.6 & PUA 10:2 & decadienal & 3.65 & 0 & -1 & 4.55 & -2.95 & & $\mathrm{C}_{10} \mathrm{H}_{17} \mathrm{O}$ \\
\hline PUA 10:1 none & 2.25 & PUA 10:2 & decadienal & 3.65 & 0 & -1 & 4.55 & -2.3 & & \\
\hline PUA 10:3 none & 1.45 & PUA 10:2 & decadienal & 3.65 & 0 & 1 & 2.75 & -1.3 & & $\mathrm{C}_{10} \mathrm{H}_{13} \mathrm{O}$ \\
\hline PUA 10:4 none & 1.52 & PUA 10:2 & decadienal & 3.65 & 0 & 2 & 1.85 & -0.33 & decatetrienal (3b) & \\
\hline PUA 11:1 none & 1.79 & PUA 10:2 & decadienal & 3.65 & 1 & -1 & 5.6 & -3.81 & & $\mathrm{C}_{11} \mathrm{H}_{20} \mathrm{O}$ \\
\hline PUA 11:2 none & 1.84 & PUA 10:2 & decadienal & 3.65 & 1 & 1 & 4.7 & -2.86 & & $\mathrm{C}_{11} \mathrm{H}_{18} \mathrm{O}$ \\
\hline PUA 11:3 none & 1.61 & PUA 10:2 & decadienal & 3.65 & 1 & 1 & 3.8 & -2.19 & & $\mathrm{C}_{11} \mathrm{H}_{16} \mathrm{O}$ \\
\hline PUA 11:3 none & 7.08 & PUA 10:2 & decadienal & 3.65 & 1 & 1 & 3.8 & 3.28 & & \\
\hline PUA 11:4 none & 1.6 & PUA 10:2 & decadienal & 3.65 & 1 & 2 & 2.9 & -1.3 & & $\mathrm{C}_{11} \mathrm{H}_{14} \mathrm{O}$ \\
\hline PUA 12:1 none & 1.96 & PUA 10:2 & decadienal & 3.65 & 2 & -1 & 6.65 & -4.69 & & $\mathrm{C}_{12} \mathrm{H}_{22} \mathrm{O}$ \\
\hline PUA 12:1 none & 3.67 & PUA 10:2 & decadienal & 3.65 & 2 & -1 & 6.65 & -2.98 & & \\
\hline PUA 12:2 none & 2.08 & PUA 10:2 & decadienal & 3.65 & 2 & 0 & 5.75 & -3.67 & & $\mathrm{C}_{12} \mathrm{H}_{20} \mathrm{O}$ \\
\hline PUA 12:3 none & 1.79 & PUA 10:2 & decadienal & 3.65 & 2 & 1 & 4.85 & -3.06 & & $\mathrm{C}_{12} \mathrm{H}_{18} \mathrm{O}$ \\
\hline PUA 12:4 none & 1.59 & PUA 10:2 & decadienal & 3.65 & 2 & 2 & 3.95 & -2.36 & & $\mathrm{C}_{12} \mathrm{H}_{16} \mathrm{O}$ \\
\hline PUA 12:5 none & 1.49 & PUA 10:2 & decadienal & 3.65 & 2 & 3 & 3.05 & -1.56 & & $\mathrm{C}_{12} \mathrm{H}_{14} \mathrm{O}$ \\
\hline
\end{tabular}


Table 5. Putative annotation of small unknown molecules that originally annotated as PUAs but did not agree with predicted retention times. The $\mathrm{m} / \mathrm{z}$ of each feature wasby querying against metabolites from KEGG pathways $( \pm 5 \mathrm{ppm})$.

\begin{tabular}{|c|c|c|}
\hline Annotation & $\begin{array}{c}\text { Structural annotation assigned } \\
\text { with custom database }\end{array}$ & Structural annotation assigned with KEGG \\
\hline $\mathrm{C}_{9} \mathrm{H}_{18} \mathrm{O}$ RT-1.90 & PUA 9:0 & None \\
\hline \multicolumn{3}{|l|}{$\mathrm{C}_{9} \mathrm{H}_{18} \mathrm{O}$ RT-3.45 } \\
\hline $\mathrm{C}_{9} \mathrm{H}_{16} \mathrm{O}$ RT- 1.80 & PUA 9:1 & None \\
\hline $\mathrm{C}_{10} \mathrm{H}_{20} \mathrm{O}$ RT-2.06 & PUA 10:0 & Decanal (PUA 10:0), Menthol, Citronellol, Neomenthol \\
\hline $\begin{array}{l}\mathrm{C}_{10} \mathrm{H}_{18} \mathrm{O} \text { RT-1.6 } \\
\mathrm{C}_{10} \mathrm{H}_{18} \mathrm{O} \text { RT-2.25 }\end{array}$ & PUA 10:1 & $\begin{array}{l}\text { (1S-2S-4S)-Dihydrocarveol, (S)-(-)-Citronellal, 1-8-Cineole, (1R-2R-4S)-Iso-dihydrocarveol, Terpineol-4, } \\
\text { (-)-endo-Fenchol, (R)-(+)-Citronellal, (+)-trans-Piperitenol, Borneol, (R)-(+)-alpha-Terpineol, DL-alpha- } \\
\text { Terpineol, (1R-2R-4R)--ihydrocarveol, beta-Terpineol, (+)-Isomenthone, (1R-2S-4S)- Neoiso- } \\
\text { dihydrocarveol, 8-p-Menthen-2-ol, Geraniol, 1-4-Cineole, gamma-Terpineol, (1S-2R-4S)-Neo- } \\
\text { dihydrocarveol, (-)-Isomenthone, 3-7-Dimethylocta-1-6-dien-3-ol, (-)-Linalool, (+)-Linalool, (-)-Borneol, } \\
\text { (+)-Borneol, (1S-2R-4R)-Neoiso-dihydrocarveol, (+)-Menthone, (1S-2S-4R)-Iso-dihydrocarveol, } \\
\text { Chrysanthemol, Citronellal, Nerol, (-)-alpha-Terpineol, Sabinene hydrate, (+)-trans-Pulegol, (1R-2S-4R)- } \\
\text { Neo-dihydrocarveol, (-)-Menthone }\end{array}$ \\
\hline $\mathrm{C}_{10} \mathrm{H}_{14} \mathrm{O}$ RT-1.45 & PUA $10: 3$ & $\begin{array}{l}\text { Piperitenone, 4-n-Butylphenol, Pinocarvone, (+)-Menthofuran, Isopiperitenone, Carvacrol, Thymol, p- } \\
\text { Cumic alcohol, Myrtenal, Chrysanthenone, Umbellulone, Menthofuran, (+)-Chrysanthenone, (+)-(S)- } \\
\text { Carvone, (+)-Sabinone, Perillyl aldehyde, 4-sec-Butylphenol, 2-sec-Butylphenol, 2-tert-Butylphenol, } \\
\text { Verbenone, Eucarvone, 3-tert-Butylphenol, 4-tert-Butylphenol, Safranal, 4-Isopropyl-3-methylphenol, (-)- }\end{array}$ \\
\hline
\end{tabular}


Isopiperitenone, (-)-Carvone

$\begin{array}{lll}\mathrm{C}_{11} \mathrm{H}_{20} \mathrm{O} \text { RT-1.52 } & \text { PUA 11:1 } & \text { None } \\ \mathrm{C}_{11} \mathrm{H}_{18} \mathrm{O} \text { RT-1.84 } & \text { PUA 11:2 } & \text { None } \\ \mathrm{C}_{11} \mathrm{H}_{16} \mathrm{O} \text { RT-1.61 } & \text { PUA 11:3 } & \text { 4-n-Pentylphenol, 4-Isopentylphenol, Jasmone, p-tert-Amylphenol } \\ \mathrm{C}_{11} \mathrm{H}_{16} \mathrm{O} \text { RT-7.08 } & \text { PUA 11:4 } & \text { None } \\ \mathrm{C}_{11} \mathrm{H}_{14} \mathrm{O} \text { RT-1.6 } & \text { Geosmin } \\ \mathrm{C}_{12} \mathrm{H}_{22} \mathrm{O} \text { RT- } 1.96 & \text { PUA 12:1 } \\ \mathrm{C}_{12} \mathrm{H}_{22} \mathrm{O} \text { RT- } 3.67 & \text { PUA 12:2 } & \text { (Z-Z-Z)-3-6-9-Dodecatrien-1-ol } \\ \mathrm{C}_{12} \mathrm{H}_{20} \mathrm{O} \text { RT-2.08 } & \text { PUA 12:3 } & \text { Propofol } \\ \mathrm{C}_{12} \mathrm{H}_{18} \mathrm{O} \text { RT-1.79 } & \text { PUA 12:4 } & \text { 4-n-hexylphenol } \\ \mathrm{C}_{12} \mathrm{H}_{16} \mathrm{O} \text { RT-1.59 } & \text { PUA 12:5 } & \text { None } \\ \mathrm{C}_{12} \mathrm{H}_{14} \mathrm{O} \text { RT- } 1.49 & \text { Capillanol }\end{array}$


Table 6. Experimental Metadata for PERMANOVA of dissolved lipidome clustering

\begin{tabular}{lllll}
\hline Treatments & Strain & NO production & Stress Factor & Grazing \\
\hline WT & WT & 0 & 0 & 0 \\
PtNOA & PtNOA & 1 & 1 & 0 \\
WT+Om & WT & 0 & 1 & 1 \\
PtNOA $+O m$ & PtNOA & 1 & 2 & 1
\end{tabular}


Table 7. Results of permutational multivariate analysis of variance of the dissolved lipidome structure with respect to experimental metadata, using ADONIS

(permutations $=999$, method $=$ Euclidean $)$. Asterisk denotes significance $(\mathrm{P}<0.1)$

\begin{tabular}{lcccccc}
\hline & $d . f$. & $S S$ & $M S$ & $F$ model & $R^{2}$ & $P$ \\
\hline Strain & 1 & 0.0672 & 0.067203 & 0.61676 & 0.05809 & 0.8264 \\
Residual & 10 & 1.0896 & 0.108962 & 0.94191 & & \\
Total & 11 & 1.1568 & & & & \\
Stress factor & 2 & 0.26945 & 0.134724 & 1.3664 & 0.23292 & 0.1983 \\
Residual & 9 & 0.88738 & 0.098598 & 0.76708 & & \\
Total & 11 & 1.15683 & & & & \\
NO production & 1 & 0.0672 & 0.067203 & 0.61676 & 0.05809 & 0.8182 \\
Residual & 10 & 1.0896 & 0.108962 & 0.94191 & & \\
Total & 11 & 1.1568 & & & & \\
Grazing & 1 & 0.22679 & 0.226795 & 2.4386 & 0.19605 & $0.03306 *$ \\
Residual & 10 & 0.93003 & 0.093003 & 0.80395 & & \\
Total & 11 & 1.15683 & & & &
\end{tabular}




\section{Chapter 4}

Distribution and abundance of oxylipins in the California Coastal System corresponded with bloom decline and the abundance of RNA viruses. 


\section{Introduction}

Oxylipins are lipid derived infochemicals hypothesized to mediate diatom bloom dynamics and influence ocean biogeochemistry. However, these hypotheses are largely based on culture studies and we are only beginning to understand oxylipin infochemical signaling in situ. Oxylipins are produced mainly by diatoms and some prymnesiophytes. When the cell integrity of these organisms is compromised, free fatty acids are cleaved from membrane lipids and nucleophilic, oxygen-containing functional groups are added by a broad array of enzymes (Andreou et al., 2009). The resulting class of molecules is bioactive, functioning as infochemical signals, and diverse, ranging from relatively large hydroperoxy acids and hydroxy epoxy acids to the smaller polyunsaturated aldehydes (PUAs) and diatom hormones (Fig 1). The $\mathrm{CO}_{2}$ fixed and subsequently exported during diatom blooms represents a significant sink in the ocean carbon cycle. The production of bioactive molecules as cells are damaged during bloom decline undoubtedly impacts the fate of this carbon.

PUAs represent the most well studied oxylipins in the marine environment and facilitate infochemical signaling between a wide range of marine organisms that are key to the cycling of carbon through the microbial loop. Largely, the effects of PUAs are deleterious and dosedependent, decreasing the reproductive success of copepods (Miralto et al., 1999), inhibiting microzooplankton grazing in culture (Chapter 3) and in shipboard experiments (Johnson et al., in prep), and decreasing the growth rates of other eukaryotic phytoplankton, microzooplankton grazers, and several bacterial isolates in culture (Lavrentyev et al., 2015; Ribalet et al., 2007a; Ribalet et al., 2008). However, particle associated communities in the North Atlantic responded to PUAs in a unique dose-dependent fashion, exhibiting increased organic matter respiration, enzymatic activity, and bacterial growth under stimulatory concentrations and decreased bacterial growth at higher concentrations (Edwards et al., 2015). In diatoms, exposure to small concentrations of PUAs 'immunizes' the population to later toxic doses, whereas, exposure to large concentrations induces apoptosis, forming a nitric oxide mediated stress surveillance system (Vardi et al., 2008; Vardi et al., 2006).

The bioactivity of PUAs has numerous implications for carbon flux through the oceans, potentially altering the standing stock of copepod grazers, the transfer of carbon to the dissolved pool by microzooplankton, phytoplankton community succession, export efficiency of sinking 
particles, and nutrient recycling by particle associated communities, just to name a few. But PUAs represent just a small proportion of oxylipin diversity. Ianora et al. (2015) found that nonvolatile oxylipins (NVOs) were well correlated with decreased hatching success of copepods in the Adriatic Sea. And the non-volatile oxylipin amendment experiments recently showed that 15HpETE and Leukotriene B4 (5,12-dihydroxyETE) decrease microzooplankton grazing of Phaeodactylum tricornutum in a dose dependent manner (Johnson et al., submitted). Therefore, non-volatile oxylipins, like PUAs, are predicted to play an integral role in diatom bloom dynamics and the biogeochemistry of the upper ocean.

To understand the ecological relevance of oxylipins in the environment, in situ measurements must be made, especially considering the dose-dependence of oxylipin bioactivity, the wide range of induced responses, and the structural diversity of the class. Even with the thrust of infochemical research in the ocean focusing on PUAs, there are currently only four published studies that measured PUA along an oceanic transect and half of those studies used sampling methods that were inappropriate for surveying dissolved oxylipins in the ocean.

Bartual et al. (2014) measured PUA production by large phytoplankton on four cruises across seven biogeographical oceanic provinces. However, this sampling method concentrated phytoplankton cells and utilized multiple rounds of sonication to elicit PUA production. Therefore, the values are reported are potential PUA concentrations and do not represent the gradient of concentrations nor the suit of compounds experienced by organisms in the environment. Vidoudez et al. (2011a) was diligent in making that distinction, presenting both dissolved PUA concentrations and potential PUA concentrations for the surface ocean of the Adriatic Sea during a Skeletonema marinoi bloom. Ribalet et al.(2014) also adopted the convention of measuring both dissolved and potential PUA concentrations in the northern Adriatic Sea. As to the diversity of oxylipins produced in situ, at ten out of twelve stations sampled in the northern Adriatic Sea by Ianora et al.(2015), higher potential concentrations of non-volatile oxylipins were observed compared to potential concentrations of PUAs. However, the authors only alluded to the diversity of compounds observed in field, noting that they measured the same oxylipins as were previously described for S. marinoi, Chaetoceros socialis, and Chaetoceros affinis cultures. 
In situ measurements of oxylipins can also be understood in an ecological and biogeochemical context by linking the presence of oxylipins to biological and chemical processes. In the northern Adriatic Sea, dissolved and particulate PUA concentrations were linked with phytoplankton lysis (Ribalet et al., 2014). It was speculated that nutrient stress, UV irradiation, viral infection, or PUAs themselves could have been the cause of phytoplankton lysis and subsequent release of PUAs but the authors were unable to test the hypotheses with their dataset. The four transects measuring PUAs from large phytoplankton across the North Atlantic showed a good correlation between N:P ratios and potential PUA concentrations (Bartual and al, 2014). Indeed culture studies have shown that nutrient stress, seasonal variability, and UV irradiation are all associated with altered PUA production upon simulated wounding (Pacheco et al., 2014; Ribalet et al., 2009; Ribalet et al., 2007b; Taylor, 2009).

However, the correlation between potential PUA concentrations and low N:P in the North Atlantic does not necessarily mean that dissolved PUA concentrations are correlated with nutrient stress. Additionally, the most likely mechanism underlying the correlation seen in the northern Adriatic Sea, viral infection, has not yet been investigated with respect to oxylipin infochemical signaling in culture. Therefore, these field studies are not sufficient enough to concretely say what environmental factors control dissolved oxylipin concentrations and infochemical signaling in the ocean.

Driven by the seemingly ubiquitous nature of oxylipin infochemical signaling in the microbial loop, the lack of measurements in the marine environment, and the implications for ocean biogeochemistry, the goals of this study were two-fold: 1) utilize novel lipidomics techniques to determine the distribution and diversity of oxylipins produced in a dynamic environment where diatoms dominated the phytoplankton community 2) link the meta-lipidome structure to biological and chemical parameters such as, nutrient concentrations, biological proxies, Si uptake measurements, enzymatic activity rates, grazing rates, and RNA viral reads measured along the cruise track. This approach revealed that free fatty acids from diatoms and non-volatile oxylipins dominated the dissolved lipid pool, the structure of the dissolved metalipidome varied significantly across the cruise track, and oxylipin abundance was strongly linked to bloom decline and viral abundance. Several novel oxylipins were observed in the dissolved lipidome. Many were relatively abundant and varied significantly across the cruise track, making 
them promising new analytes. Additionally, a role for oxylipins in balancing mechanisms of diatom mortality was proposed.

\section{Materials and Methods}

\subsection{DYEatom cruise specifics and supplemental data collection}

The DYEatom cruise disembarked from Moss Landing, CA on July $27^{\text {th }}, 2013$. The cruise track transected the California coastal upwelling region, as well as, an offshore eddy at $37^{\circ} 33.42^{\prime} \mathrm{N} x 123^{\circ} 37.96^{\prime} \mathrm{W}$ (Figure 2). Along the cruise track, nine stations were occupied. A CTD rosette was used to collect water from six depths between the surface ocean $(\sim 2 \mathrm{~m})$ and 50m. Seven core biological and chemical parameters were measured at each depth:, total chlorophyll, chlorophyll a, biogenic $\mathrm{Si}$, phaeophytin, dissolved $\mathrm{Si}$, nitrate and nitrite, and soluble reactive phosphate (SRP) (Fig 3A-I). Nutrients and pigments were measured at the University of California-Santa Barbara Marine Science Institute Analytical lab. With the exception of Stations 1 and 11, enzymatic activities of leucine, serine, and glycine peptidases and alkaline phosphatase (APase) were measured at each sampling depth using fluorogenic model substrates (Hoppe, 1993) (Fig 4A-D).

At the 55\%, 22\%, and 1\% incident irradiance depths (Io), samples were collected for metatranscriptomic analysis on $1.2 \mu \mathrm{m}$ filters which were frozen at $-80^{\circ} \mathrm{C}$ and transferred back to Rutgers University. RNA was purified, treated with DNase and the RNAeasy MinElute Kit (Qiagen) before reverse transcription and sequencing with Illumina according to a method modified from Marchetti (2012). The reads were BLAST-ed against an in-house database containing all known RNA viral genomes with a cut off 1e-05 (Fig 4F-I). There were 112 hits to RNA viral genomes with percent identity ranging from 22 to 57\%. Table 2 contains best hit annotations for the RNA viral meta-transcriptome. The meta-transcriptomic work was done as part of a collaboration with Kim Thamatrakoln (Rutgers), Kay Bidle (Rutgers) and Andy Allen (J. Craig Venter Institute). Silica stress was measured throughout the light upper water column along the cruise track using a ${ }^{32} \mathrm{Si}$ isotope uptake method to compare the 24-hour uptake rates under ambient Si concentrations $\left(\mathrm{V}_{\mathrm{amb}}\right)$ to uptake rates after spiking the incubation with $20 \mu \mathrm{M}$ silicate $\left(\mathrm{V}_{\text {sat }}\right)($ Krause et al., 2011). Dilution experiments $(5,20$, and $100 \%$ whole seawater) were 
conducted in the surface ocean $(\sim 2 \mathrm{~m})$ at 8 of the 9 stations to determine microzooplankton grazing rates (Johnson et al., submitted; Landry and Hassett, 1982) (Fig 5A). Phytoplankton growth rates were also determined in these experiments by measuring changes in chlorophyll (Fig 5B). Additionally, the dissolved lipidome was sampled at the 55\% Io depth and at the chlorophyll maximum at the nine stations (method described below). This information is consolidated in Table 1.

\subsection{Dissolved lipidome sampling}

The dissolved lipidome was sampled at two depths in the water column along the cruise track, the 55\% Io depth and the chlorophyll maximum. One liter of seawater from each depth was filtered through a bottle top $0.2 \mu \mathrm{m}$ filter (Corning) to remove particulate material. The internal standard benzaldehyde (Sigma-Aldrich) was added to a final concentration of $10 \mu \mathrm{M}$ in one liter of seawater. Using a vacuum manifold, the samples were filtered onto a Water HLB solid phase extraction (SPE) cartridge for approximately one hour. After one hour the remaining sample was measured in a graduated cylinder and the filtered volume was recorded. The SPE cartridges were acidified with $0.1 \%$ hydrochloric acid, placed in a whirl-pak bag, and stored in liquid nitrogen. At the end of the cruise the samples were transported back to Woods Hole in a dry shipper where they were stored at $-80^{\circ} \mathrm{C}$ until elution.

SPE cartridges were removed from the $-80^{\circ} \mathrm{C}$ freezer and allowed to thaw for 5 minutes before elution. SPE cartridges were rinsed with $2 \mathrm{ml}$ of milli-Q water to remove any excess salt. The antioxidant BHT was added to the pre-combusted collection vials before elution to prevent autoxidation of the samples. The samples were eluted from the SPE cartridge with $2 \mathrm{ml}$ of methanol. The samples were immediately transferred from the collection vials into HPLC vials and capped under argon. The samples were stored a $-80^{\circ} \mathrm{C}$ until mass spectrometric analysis. Of the eighteen dissolved lipidome samples collected, only sixteen were extracted, as two were lost in transit. 


\subsection{Dissolved lipidome analysis}

Dissolved lipidome samples were analyzed in positive and negative mode using reverse phase HPLC (Agilent 1200 system ; Agilent, Santa Clara, CA, USA) paired with high resolution, accurate mass (HRAM) data from a Thermo Exactive Plus Orbitrap mass spectrometer (ThermoFisher Scientific, Waltham, MA, USA). This method was adapted from Hummel et al. (2011) using the same chromatography gradient and injecting $20 \mu \mathrm{L}$ of sample onto a C8 Xbridge HPLC column (particle size $5 \mu \mathrm{m}$, length $150 \mathrm{~mm}$, width $2.1 \mathrm{~mm}$; Waters Corp., Milford, MA, USA). Eluent A was water with $1 \% 1 \mathrm{M}$ ammonium acetate and $0.1 \%$ acetic acid, Eluent B was $70 \%$ acetonitrile and $30 \%$ isopropanol with $1 \% 1 \mathrm{M}$ ammonium acetate and $0.1 \%$ acetic acid. The starting gradient was $45 \%$ Eluent $\mathrm{A}$ and $55 \%$ Eluent B. The gradient shifted to $1 \%$ Eluent $\mathrm{A}$ and $99 \%$ Eluent B over the 30 minute run. The first minute of each run was diverted to waste. Full scan and all-ion-fragmentation data (m/z 100-1500) was collected in positive and negative mode at a mass resolution of 170,000 on the Orbitrap mass spectrometer collected from 1 to 30 minutes. Only the negative mode data was presented here since the molecules of interest, free fatty acids and oxylipins, both form negative ions under electrospray ionization. However, the recovery efficiency of the internal standard, benzaldehyde, was extracted from the positive mode data.

Additionally, authentic standards of nineteen oxylipins were purchased from Cayman Chemical (Ann Arbor, MI), diluted in methanol to a final concentration of $10 \mu \mathrm{M}$, and analyzed using the above method. The authentic oxylipin standards were: 15(S)-HpETE (15Shydroperoxy-5Z,8Z,11Z,13E-eicosatetraenoic acid), 14,15-DiHETE (14,15-dihydroxy6Z,8E,10E,14Z-eicosatetraenoic acid), 15-HETE (15-hydroxy eicosatetraenoic acid), 14(15)EpETE (14(15)-epoxy-eicosatetraenoic acid), a mix of 5-,12-, and 15-HpETE, 15-HEPE (15hydroxy eicosapentaenoic acid), 15-HETrE (15-hydroxy eicosatrienoic acid), 9-hydroperoxy octadecadienoic acid (9-HpODE), 13-hydroperoxy octadecadienoic acid (13-HpODE), 9hydroxy octadecatrienoic acid (9-HOTrE), 13-hydroxy octadecatrienoic acid (13-HOTrE), 9hydroperoxy octadecatrienoic acid (9-HpOTrE), 13-hydroperoxy octadecatrienoic acid (13HpOTrE), 9-keto octadecatrienoic acid (9-KOTrE), octanoic acid, and 2E,4Z-decadienal. The retention times of these standards can be found in Tables 4A, 5A, 11, and 12 . 


\subsection{Dissolved lipidome annotation}

The dissolved lipidome was annotated in the software package MAVEN (Metabolic Analysis and Visualization ENgine) which detected peaks and assigned putative structural level annotations to each feature by querying a custom lipid database containing the various adducts of intact polar lipids, fatty acids, triacylglycerols, and their oxylipin derivatives (Collins et al., in prep; Melamud et al., 2010). MAVEN detected 4460 features and assigned putative structural level annotations to 2030 features (Table 3). Putative identities were only assigned to features that were greater than $10^{5}$ intensity units with at least two peaks across all samples and at least one peak with a maximum quality score of 0.8. Putative identities assigned in MAVEN were based solely on $\mathrm{m} / \mathrm{z} \pm 2 \mathrm{ppm}$ for fatty acids and NVOs and $\mathrm{m} / \mathrm{z} \pm 5 \mathrm{ppm}$ for PUAs and small fatty acids. A maximum of 3 best matches were retained for each feature. For fatty acids, NVOs, and PUAs, only $[\mathrm{M}-\mathrm{H}]^{-}$adducts were considered. The data set was reduced to the 361 features that were putatively identified as free fatty acids or their oxylipin derivatives. Lastly, the odd fatty acids were then removed from the lipidome, as well as peaks that did not meet the broad retention time criteria ( 1 minute $<\mathrm{RT}<18$ minute). There were 265 featured retained after applying this data reduction protocol.

\subsubsection{Functional group level annotation based on retention times of the standards}

Authentic standards were available for several C20 and C18 oxylipins (Table 4A and Table 5A). Comparisons between observed retention times and the retention times of the standards were used to further elucidate structural isomers and assign oxylipin functional group level annotations to some non-volatile oxylipins. Authentic standards were used to annotate the C20:5 oxylipin hydroxy eicosapentaenoic acid (HEPE RT-4.6), four C20:4 oxylipins, two C20:3 oxylipins, and five C18:n oxylipins (Table 4B and Table 5B). The confidence ranking scheme laid out in Chapter 3 was applied to annotation of this dataset. Thus compounds matching the exact mass and RT of authentic standards were considered level 2 annotations, representing the highest level of confidence achieved with the mass spectrometric method used in this study. The letter "a" after the level of confidence denoted that the $\mathrm{RT}_{\text {obs }}$ was exactly equal to the $\mathrm{RT}_{\text {auth }}$. The letter " $b$ " after the level of confidence denoted that the $\mathrm{RT}_{\text {obs }}$ was within 0.7 min of the $\mathrm{RT}_{\text {auth }}$ for $-2 \mathrm{O}$ NVOs or 0.5 min of the $\mathrm{RT}_{\text {auth }}$ for -10 NVOs, fatty acids, and PUAs. 
The differences between retention times of the C20:5 and the C20:4 hydroxy acid standards, 15-HEPE (RT- 4.6) and 15-HETE (5.4), suggested that the additional double bond increased the polarity of the oxylipin and shifts the retention time of HEPE forward 0.87 minutes. This relationship was used to determine theoretical retention times $\left(R_{+D B}\right)$ for the $C 20: 5$ analogs of the C20:4 oxylipin standards (Table 6). There were four structural isomers annotated as NVO C20:5 -2O in the meta-lipidome (Table 7). Three of the NVO C20:5 -2O isomers were further resolved as two di-hydroxy eicosapentaenoic acids (diHEPE RT-2.1 and diHEPE RT-2.9) and a hydroperoxy eicosapentaenoic acid (HpEPE RT-4.9). The NVO C20:5 20 isomers with retention times at 6.7 and 7.5 minutes eluted much later than any of our authentic $\mathrm{C} 20: 42 \mathrm{O}$ standards and were not assigned a functional group level annotation. Similarly, the NVO C20:5 10 isomers with retention times at 5.9 and 7.1 minutes eluted much later than any of our C20:4 10 standards and were not assigned a more detailed annotations. Functional group annotations based on predicted RT from authentic standards represented level 3 in our confidence ranking system. Again the "a" and " $b$ " notation is used to denote exact matches to the predicted RT and matches within the acceptable window, respectively.

A similar framework was applied to the C16:n NVO annotations. The difference between the retention times of C20:3 and C18:3 structural analogs, 15-HETrE (RT-6.1) and 13-HOTrE (RT-4.0), was 2.1 minutes, suggesting that RT increase 1.05 minutes per carbon. Using that relationship retention times of the C18:n standards were used to calculate theoretical retention times for $\mathrm{C} 16: \mathrm{n}$ analogs $\left(\mathrm{RT}_{\mathrm{C} 18->\mathrm{C} 16}\right.$; Table 8). The theoretical $\mathrm{RT}_{\mathrm{C} 18->\mathrm{C} 16}$ were compared to observed retention times for structural isomers to the C16:n analogs in the DYEatom dissolved lipidome using percent error from the observed retention times (Table 9). Three C16 oxylipins were putatively annotated to the functional group level using this method: hydroperoxy hexadecadienoic acid (HpHDE RT-3.4), hydroperoxy hexadecatrienoic acid (HpHTrE RT-2.5), and keto hexadecatrienoic acid (KHTrE/HHTE RT-2.1).

The offset in the retention times of 13-HpODE and 13-HpOTrE (0.79 minutes) was used to determine how unsaturation affects smaller chain oxylipins. This 0.79 minute decrease in retention time with increased unsaturation was applied to the theoretical RT of 16:n analogs to calculate retention times for 16:n-1, C16:n+1 and C16:n-2 analogs of C18:n standards (Table 8). The theoretical $\mathrm{RT}_{-\mathrm{DB}}, \mathrm{RT}_{+\mathrm{DB}}$, and $\mathrm{RT}_{-2 \mathrm{DB}}$ were compared to observed retention times for 
structural isomers to the C16:n-1, C16:n+1, and C16:n-2 analogs in the DYEatom lipidome (Table 10). The putative annotation of NVO 16:2 2O RT-3.4 as HpHDE was supported by comparisons to both RT C18->C16 and RT-DB calculations (Table 9 and Table 10A).

Additionally the double bond method led to the putative annotation of nine other C16 oxylipins to the functional group level. There were two lines of evidence for the annotation of NVO 16:1 $2 \mathrm{O}$ RT-4.6 as a hydroperoxy hexadecaenoic acid (HpHME), stemming from the theoretical retention times of the C16:n-1 analog of the C18 standard HpODE and the C16:n-2 analog of the C18 standard HpOTrE (Table 10A and 10C).

The RTs of the authentic standards decadienal (PUA 10:2) and octanoic acid (FFA 8:0) were also used to further annotate the molecular species annotated as PUAs and C10 fatty acids at the structural level (Table 11 and 12). Two C10 fatty acids were putatively annotated as decatrienoic acid RT-1.9 and decanoic acid RT-4.3. Two PUAs were putatively annotated as decatetrienal RT-1.7 and dodecatrienal RT-4.9, were confirmed by RT predicted from the decadienal authentic standard. The PUAs that did not match the retention times predicted by authentic standards were re-annotated at the elemental formula level because of the overlap in $\mathrm{m} / \mathrm{z}$ of PUAs with many small metabolites (See Chapter 3 Table 5). Elemental formulas represent the lowest level of annotation (5). Structural level annotations without a confidence ranking associated with it were considered level 4 annotations.

\subsubsection{Functional level annotation based on theoretical retentions calculated from octanol-water partition coefficients}

A second approach was tried to resolve the functional group level annotations of oxylipins in the meta-lipidome for which there were not appropriate standards and to resolve the positional level annotations of oxylipins with functional group annotations, employing the relationship between octanol-water partition coefficients, polarity, and retention time. The octanol-water partition coefficient of each of the standard was calculated and regressed against the retention times of the C20 and C18 oxylipins separately (Table 4A and Table 5A). The regression was used to predict the retention times of the standards $\left(\mathrm{R}_{\mathrm{Log}}\right)$ demonstrating absolute differences in RT between 0.02 and 0.94 minutes for C20 standards and between 0.01 and 1.26 absolute percent error for C18 standards (Table 4A and 5A). The C20 model did not predict the 
retention times of positional isomers with the function group close to the carboxylic acid (i.e. 5HpETE off by -0.94 minutes), epoxy acids (14,15 EpETE off by 0.68 minutes), or the HpETE positional isomers (which demonstrated an unusual elution pattern) with high accuracy. Whereas, the C18 regression did not predict the keto-acid, 9-KOTrE with high accuracy (-1.26 min offset). The molecule 9-KOTrE was eventually excluded from the calculation since it severally reduced the $\mathrm{R}^{2}$ value of the linear regression from 0.98 to 0.42 . The octanol-water partition coefficients of several positional isomers proposed for the $\mathrm{C} 20: 51 \mathrm{O}$ and $2 \mathrm{O}$ oxylipins were used to calculate theoretical retention times $\left(\mathrm{RT}_{\mathrm{LogP}}\right)$ which was compared to $\mathrm{R}_{\mathrm{obs}}$ (Table 10). Because of the poor predictive ability of the regression for epoxy and oxo acids along with the lack of authentic standards for some classes of oxylipin and the overlap of RT for oxo and hydroxy acids, this information was not conclusive enough to aid in additional annotation of the meta-lipidome.

\subsection{Normalization of the lipidome}

The lipidomic data from each sample was normalized to the volume filtered and to the internal standard. During sample collection, the internal standard was not added to the lipidomic samples from St2. And in samples, St1-5m, St7-2m, and St11-1m, the recovery of the internal standard was low, $<10 \%$. Preliminary exploratory analysis revealed that two of the three low recovery samples (St1-5m and St7-2m) drove the Principal Component Analysis (PCA) (data not shown). Therefore, these four samples were excluded from the PCA and subsequent analyses.

\subsection{Lipidomic analysis}

2.6.1 Richness and Diversity of the oxylipidome at each station.

The richness of molecular species positively annotated as free fatty acids or oxylipins was determined by summing the number of features in each sample (Fig 6A \& B). The proportion of the meta-lipidome total peak area that each molecular species contributed $\left(\mathrm{p}_{\mathrm{i}}\right)$ was calculated and used to determine the Shannon-Wiener index of diversity, H (Equation 1). 


$$
H=-\sum_{i=1}^{i} p_{i} \ln p_{i} \quad(\text { Equation } 1)
$$

\subsubsection{PCA analysis}

Covariance principle component analysis was used to determine if there were biological and chemical factors that explain the variance in the lipidome across stations (Table 14; Figure 8). Covariance PCA was chosen over correlation PCA, since all of the active variables were measured in the same units (peak area). The core biological and chemical parameters served as the supplemental variables which do not affect the principal components. PCA was generated in STATISTICA. The PC1 and PC2 loading of the cases were linearly regressed against non-core variables, to determine the significance of relationships that could not be tested with PCA due to missing values in the supplemental data (P-value of F-test of overall significance $<0.10$; Table $15)$.

\subsubsection{Similarity profile analysis}

Level scaling was applied to the data by dividing each peak area, $\mathrm{x}_{\mathrm{j}}$ by the average peak area of that feature across the samples, $x_{\text {avg }}$ (Equation 2). Euclidean distance matrices were created for the level scaled data and used to construct a dendrogram describing the hierarchical clustering of the samples and the compounds (Figure 9). Similarity profile analysis was then employed to determine which samples had significantly similar lipidomes and which compounds varied similarity across samples (Table 16). The SIMPROF function in clustsig R package was used.

$$
x_{\hat{\jmath}}=\frac{x_{j}}{x_{a v g}} \quad(\text { Equation 2) }
$$

\subsubsection{Permutational multivariate analysis of variance}

A permutational multivariate analysis of variance (PERMANOVA) was conducted to determine which supplemental variables were most attributable to the hierarchical clustering of the samples. The ADONIS function in the R package vegan was used (Anderson, 2001). Only core biological and chemical variables were tested since the analysis required complete datasets without missing variables. All core variables were analyzed together in one test that assumed an additive relationship (i.e. the dissimilarity of the distance matrix $\sim$ total chlorophyll +chlorophyll 
$\mathrm{a}+$ phaeophytin $+\mathrm{bSi},+\mathrm{dSi},+\mathrm{SRP},+$ nitrogen) (Table 17). The core variables were also considered separately but no significant relationship were observed.

\section{Results}

\subsection{Cruise description and biogeochemical properties across the transect}

From June $27^{\text {th }}$ to July $5^{\text {th }}, 2013$ the DYEatom cruise abroad the R/V Point Sur (PS1312) traversed the productive coastal California upwelling region, as well as, an eddy off of the continental shelf (Figure 2). Nine stations were occupied along the cruise track. Stations 1, 2, 8, 10 and 11 were in the coast upwelling region. Stations 6, 7 and 9 were off of the continental shelf with Station 7 corresponding to an anticyclonic eddy. Information on parameters measured at each station can be found in Table 1 .

A dynamic nutrient field, as well as, large variations in phytoplankton biomass, RNA virus metatranscriptomic reads, grazing, Si-stress, and enzymatic activities were observed along the cruise transect. Stations 2 and 6 were the most nutrient replete (Fig 3 F, G, and H). The ratio of N:P deviated the most from Redfield in the surface ocean at Stations 4, 8, 9, and 10 (Fig 3 I). The most silica stressed stations were Stations 1, 6, 10 and 11 (Fig 4E). Phytoplankton biomass, as assessed by total chlorophyll concentrations, was fairly high across the cruise transect (Fig 3 B). While diatom biomass, as assessed by chlorophyll a and biogenic Si concentrations, was highest in the surface ocean at Station 9 and in the subsurface at St 10 (Fig 3C). The highest phaeophytin concentrations were also observed in the surface ocean at Station 9 and in the subsurface at St 10 (Fig 3D). Microzooplankton grazing rates were above $1 \mathrm{~d}^{-1}$ at Stations 1, 2, and 8 (Fig 5A). The highest growth rates were observed at St 1,2,7, and $8\left(>1 \mathrm{~d}^{-1}\right)$ and the lowest growth rates were observed at stations 10 and $9\left(<0.25 \mathrm{~d}^{-1}\right)$ (Fig 5B). Stations 7 and 10 had the highest RNA viral reads with a subsurface maximum at Station 7 and a surface maximum at Station 10 (Fig 4E). Phytophthora infestans RNA virus 1 dominated the viral community at both stations (Fig 4F). Stations 7 and 10 had the largest dissolved lipidome peak area and the highest molecular species richness (Fig $6 \mathrm{~A}$ and 6C). However, the meta-lipidomes at Stations 7 and 10 did not demonstrate higher molecular species diversity (Fig 6B). 


\subsection{Lipidomic analysis}

\subsubsection{Most abundant compounds in the dissolved lipidome}

There were 265 compounds annotated as oxylipins and free fatty acids from the database (Table 3), represent $86 \%$ of the annotated peak area. This subset of compounds will be referred to as the dissolved lipidome or dissolved meta-lipidome. There were twenty molecular species that were more than $1 \%$ of the average total peak area of dissolved lipidome, constituting about $70 \%$ of the peak area in each sample (Fig 7). The C20:5 and C16:4 free fatty acids, eicosapentaenoic acid (EPA) and hexadecatetraenoic acid (HTA), dominated the dissolved lipidome across the cruise track averaging $17.7 \%$ and $13.2 \%$ of the total peak area (Fig 7). The short chain polyunsaturated free fatty acid, decatrienoic acid (FFA C10:3 RT-1.9), was the third most abundant molecule in the dissolved meta-lipidome (6.6\%). The free fatty acids, docosahexaenoic acid (DHA; FFA C22:6 RT-8.2), hexadecatrienoic acid (FFA C16:3 RT-6.8), hexadecadienoic acid (FFA C16:2 RT-7.5), octadecapentaenoic acid (FFA C18:5 RT-6.7), octadecatetraenoic acid (FFA C18:4 RT-7.3) and octadecaenoic acid (C18:1 RT-9.3), were also observed amongst the twenty most abundant molecular species. The percentage of the peak area attributable to all of the features in the dissolved lipidome can be found in Fig 7B.

One fifth of the twenty most abundant molecules in the dissolved meta-oxylipidome were annotated as C20:5 derived oxylipins which were further resolved to the oxylipin functional group level using retention time information from C20:n standards (Table 4 and 5). Hydroxy eicosapentaenoic acid (HEPE RT-4.6) was the most abundant oxylipin in the meta-lipidome constituting an average of 3.6\% of the total peak area (Fig 7). Di-hydroxy eicosapentaenoic acid (diHEPE RT- 2.1) and hydroperoxy eicosapentaenoic acid (HpEPE RT-4.9), were the $5^{\text {th }}$ and 7 th most abundant molecules in the dissolved lipidome making up an average of $2.7 \%$ and $2.34 \%$ of the total peak area. Another isomer of diHEPE was present in the meta-lipidome, eluting at 2.9 minutes and comprising $1.39 \%$ of the total peak area. Oxylipins annotated as C16 hydroperoxy acids were also relatively abundant in the meta-lipidome with HpHME and HpHTrE making up 1.3 and $1.2 \%$ of the total peak area. Two other C16 oxylipins, NVO 16:2 1O RT-4.6 and NVO 16:1 104.3 , constituted 1.7 and $1.2 \%$ of the meta-lipidome. However, the functional group level annotation of these molecules could not be resolved using the C18 standards as they eluted much later than the theoretical retention times would predict (Table 10). 


\subsubsection{Descriptive analyses of the dissolved lipidome}

\subsubsection{Significantly varying compounds in the dissolved lipidome}

Principal component analysis was applied to the meta-lipidomic data to determine if any of the biological or chemical parameters measured across the cruise track explained the variations observed in the data. Since the active variables (free fatty acids and oxylipin peak areas) were reported in the same units, covariance PCA was applied (Abdi, 2010). Principal component 1 (PC1) represented $83.44 \%$ of the variability in the dataset, whereas, PC2 represented 9.38\% (Figure 8). The bulk of molecular species loaded together on PC1 and PC2 (Fig 8A). However, there were nineteen compounds that plotted separately from the majority of the dataset and were considered to be significantly varying compounds. Sixteen of these compounds were also the most abundant molecular species across the cruise track (Fig 7). The most abundant compound in the meta-oxylipidome, C20:5 free fatty acid contributed the most to PC1 (54\%) followed by the diHETE isomer at 2.1 minutes (11.5\%) (Fig 7; Table 14B). The second most abundant compound in the meta-oxylipidome, C16:4 only contributed $3.7 \%$ to PC1 but contributed 39\% to PC2. The diHETE isomer at 2.1 minutes contributed 21\% to PC2, whereas, the $\mathrm{C} 20: 5$ free fatty acid contributed $12 \%$. Covariance PCA weights the analysis towards variables with the most variation and thus the most abundant molecules in the dissolved meta-oxylipidome demonstrated the most variation and drove the PCA analysis.

\subsubsection{Principal component loadings of core supplemental variables underlying large variation} in the dissolved lipidome

The core biological and chemical parameters measured along the transect were included as supplemental variables in the PCA (Fig 8B). Based on the loading of the supplemental variables, $\mathrm{PC} 1$ appeared to describe the presence of phaeophytin and bSi in the absence of nitrogen, $\mathrm{dSi}$, and chlorophyll a. The supplemental variables did not load as strongly on PC2. However phaeophytin and bSi also loaded opposite of $\mathrm{Chl}$ a, dSi, and nitrogen on PC2. The cosine squared values generated during PCA represent the importance of a principal component for a given observation. For PC1 and PC2, phaeophytin and dSi have the highest cosine squared values, respectively (Table 14C). 
The nineteen significantly varying compounds loaded negatively on PC1 with the supplemental variables phaeophytin and bSi suggesting a link between the variation of these compounds and the degradation of diatom biomass (Fig 8A). Five of the significantly varying compounds loaded negatively on PC2 away from the main cluster of molecules. All five compounds were free fatty acids (C20:5, C16:4, C18:5,C18:4, and C16:3). Conversely, nine of the ten significantly varying compounds that loaded positively on PC2 were non-volatile oxylipins. The one compound positively loading on PC2 that was not a NVO was the C10:3 free fatty acid. This suggests that un-oxidized free fatty acids were associated with higher concentrations of $\mathrm{dSi}$ and oxylipins with lower dSi concentrations.

Principal Component 1 separated Stations 7 and 10 from the rest of the samples (Fig 8C). PC2 resolved differences in Stations 6, 8, 9 and 10 from Stations 1, 4, 7 and 11. St10-10m had the largest contribution to PC1 (68\%), whereas, St7-8m contributed the most to PC2 (18.5\%) (Table 14). However St11-2m, St10-10m, St4-9m, and St1-10m also contributed more than 10\% to PC2. The meta-lipidomes of St 6-4m, St6-35m, St 8-4m and St 9-7m loaded similarly on PC1 and PC2, forming the tightest cluster of samples. Taking all of this information together the PCA of the meta-lipidome mainly describes the link between phaeophytin and the nineteen significantly varying compounds which is largely driven by St10. Underlying the relationship between large variations in the meta-lipidome and phaeophytin, is another relationship describing the variability of oxylipins relative to free fatty acids which is loosely linked to dSi and driven by St 7 .

\subsubsection{Correlations between non-core supplemental variables and the principal components} describing the dissolved lipidome revealed relationship with RNA viral abundance

There were several other supplemental variables that were not measured at all of the depths and therefore could not be included in the PCA (Table 1). Si-stress was measured throughout the water column along the cruise track with values for ten of the eleven depths where quality metalipidomic data was generated. Enzymatic activity of leucine-amino peptidase, serine-amino peptidase, glycine-amino peptidase, and APase were also measured throughout the cruise however only nine measurements lined up with the eleven analyzed meta-lipidomes. Viral reads were measured at three depths at each station $(55,22$, and $1 \%$ Io) which corresponded to eight depths were the meta-lipidome was also analyzed. Grazing and 
phytoplankton growth rate were measured in the surface ocean at each station providing direct grazing measurements for four of the eleven meta-lipidome samples.

While the incomplete supplemental data sets could not be directly considered in the PCA analysis, these variables were regressed against the loadings of the samples on PC1 and PC2 (Table 15). The relationship between PC1 and the core supplemental variables, phaeophytin and $\mathrm{bSi}(\mathrm{F}=0.090$ and 0.063$)$ was confirmed using this approach but the relationship between PC2 and $\mathrm{dSi}$ was not confirmed $(\mathrm{F}=0.457)$. Furthermore, regression analysis revealed significant correlation of PC1 with total RNA viral reads $(\mathrm{F}=0.008)$. The ratio of $\mathrm{N}: \mathrm{P}$ was also significantly correlated with the loading of samples on PC2 $(\mathrm{F}=0.075)$ along with the proportion of the RNA viriome attributable to Phytophthora infestans RNA virus 1, Marine RNA virus JP-A, and Chaetoceros sp. RNA virus $2(\mathrm{~F}=0.053,0.002$, and 0.001).

\subsubsection{Level Scaling of the data and over all shifts in the dissolved lipidome}

Since the molecules present in the high abundances drove the PCA analysis, level scaling was applied so the high and low abundance molecules could be considered equally (Fig 9B). Hierarchical cluster analysis was then applied to the data to determine similarities between the meta-lipidomes of the various samples and to determine which compounds varied similarly across samples. Similarity profile analysis was used to determine which branches of each dendrogram were significantly similar to one another (Fig 9A and 9C).

The eleven meta-lipidomes formed 6 distinct clusters with two branches (Fig 9A). The first branch was formed by St9-7m, St8-4m, St6-35m and St6-4m. The dissolved meta-lipidomes from St6-4m. St6-35m, and St8-4m were significantly more similar than that of St9-7m, forming two distinct clusters (Group I and Group II). The second branch contained four distinct clusters (Group III-VI). The meta-lipidomes of St7-8m and St10-10m were significantly similar to one another (Group III) and only about 20\% similar to other Groups in the second branch. St8-1m was not significantly similar to any other samples, forming Group IV, a singlet branch in the dendrogram that is about $35 \%$ similar to Groups V and VI. The meta-lipidomes of St 1-10m and St 4-9m formed Group V and Group IV consisted of St11-2m and St 4-2m.

The 265 compounds within the meta-lipidome were grouped into 2 branches of 40 significantly clustering groups (Fig 8C; Table 16). The most abundant molecules were dispersed 
amongst ten significantly clustering groups of molecules (denoted by Arabic numerals in Table 16). Groups 36, 37, and 38 represented molecules that were only present at St7 and St10 and contained molecules annotated as the C12:3 PUA dodecatrienal RT-4.9 (3b) and a small lipophilic molecule with the elemental formula $\mathrm{C}_{10} \mathrm{H}_{14} \mathrm{O}$ RT-4.9. Group 2 molecules were generally only found at St7, St10 and St8-1m. Molecules in Group 6 were only present in St7, while molecules in Group 1 were mainly present in St10.

Similar to PCA, permutational multivariate analysis of variance (PERMANOVA) was used to determine if any of the supplemental variables adequately described the similarities in meta-oxylipidomic structure across the samples (Table 17). Separately none of the core variables were significantly correlated to the lipidomic structure of the samples. However, when all of the core variables were considered together, phaeophytin was most attributable to the similarities amongst stations, re-enforcing the view that oxylipidomic structure is linked to degradation of diatom biomass.

\section{Discussion}

\subsection{Strong contribution from diatoms to the dissolved meta-lipidome}

\subsubsection{Diatom derived free fatty acids}

Canonical diatom fatty acids and non-volatile C20 oxylipins were the most abundant molecules in the meta-lipidome across the DYEatom cruise transect, suggesting diatom oxylipin biosynthesis contributed greatly to the dissolved lipid pool in the Coastal California System (CCS). Eicosapentaenoic acid (EPA; FFA C20:5 RT-7.7) and hexadecatetraenoic acid (HTE; FFA C16:4 RT-5.7) were the two most abundant molecules in the meta-lipidome constituting an average of $17.7 \%$ and $13.2 \%$ of the total peak area, respectively (Fig 7). EPA is generally the dominate fatty acid in diatoms, making up an average of $22 \%$ of cellular fatty acids in centric and pennate diatoms (Dunstan et al., 1994). While the C16:4 fatty acid is common in the lipidome of diatoms it generally constitutes a much smaller percentage of total fatty acids within diatom cells ( 4\%, Dunstan et al., 1994). Only the C16:3, C16:4, C20:4, C20:5, and C22:6 fatty acids are thought be used as substrates for oxylipin biosynthesis in diatoms (Andreou et al., 
2009; Nanjappa et al., 2014). Thus the disproportionate abundance of the C16:4 free fatty acid in the lipidome relative to its cellular abundance is likely due to lipolytic cleavage of C16:4 fatty acids from intact polar lipids during oxylipin biosynthesis. Two of the other fatty acids favored for oxylipin biosynthesis, C16:3 and C22:6 were also relatively abundant within the metalipidome, constitution $1.7 \%$ and $1.5 \%$ of the average total peak average. Finding lipid biomarkers for diatoms in the dissolved meta-lipidome is consistent with Chaetoceros and Pseudo-nitzchia sp. dominating the phytoplankton community at most of the DYEatom cruise stations (Krause and Thamatrakoln, personal communication).

Interestingly arachidonic acid (C20:4) was not observed in the meta-lipidome. Taken on its own, this is not surprising. C20:4 fatty acids make up less than 1\% of the cellular free fatty acids in diatoms (Dunstan et al., 1994). But twelve molecular species were annotated as C20:4 oxylipins, suggesting that that C20:4 was used as a substrate for oxylipin biosynthesis (Figs 7B and 9B). A feature annotated as a C20:4 non-volatile oxylipin with three oxygens (NVO C20:4 30 RT-1.7) was the $6^{\text {th }}$ most abundant compound in the dissolved lipidome (Fig 7). Oxylipins with three oxygens are rare in diatoms and were only recently reported for P. tricornutum in Chapter 3 of this thesis. Structural isomers to NVO C20:4 3O RT-1.7 exist in mammalian systems. Lipoxin A4 and lipoxin B4 are trihydroxy acids that function as short lived inflammation signals in human cells, signaling macrophages to phagocytose the remains of cells that have undergone apoptosis (Mitchell et al., 2002). Lipoxins also facilitate communication between several cell types in the human reproductive, respiratory, and immune systems in a dose dependent manner (Chiang et al., 2005; Levy et al., 1993; Papayianni et al., 1996). Given the anti-inflammatory nature of lipoxins in mammals and the link to apoptosis, trihydroxy acids should be added to the suite of authentic standards and used to further elucidate the structure of -30 NVOs which may function as infochemical signals in diatom bloom communities.

\subsubsection{High abundance of non-volatile oxylipins derived from C20:5}

Many of the most abundant molecules in the meta-lipidome were C20:5 oxylipins accounting for approximately $10 \%$ of the total peak area of the meta-lipidome. The EPA oxylipin pathway represents the most common and the most diverse diatom oxylipin pathway with 
twenty-eight molecular byproducts observed in across 14 diatom species in culture (Fig1), perhaps explaining the prevalence of C20:5 oxylipins in the dissolved pool. Hydroperoxy eicosapentaenoic acid (HpEPE) is the first oxylipin produced after lipolysis of EPA from intact membrane lipids and HpEPE RT-4.9 made up an average of $2.3 \%$ of the peak area in the dissolved meta-lipidome (Fig 7). Peroxidation of HpEPE produces hydroxy-eicosapentaenoic acid (HEPE) which was the most abundant oxylipin in the meta-lipidome constituting an average of $3.6 \%$ of the dissolved meta-lipidome peak area. Dihydroxy eicosapentaenoic acid (diHEPE) was present as two isomers constituting $2.7 \%$ and $1.4 \%$ of the lipidome on average. The production of diHEPE in diatoms was first reported for $P$. tricornutum in Chapter 3 . The production of the C20:4 analog, diHETE, has been studied in red algae where three positional isomers with three distinct biosynthetic pathways are known (Garscha et al., 2007b; Jiang and Gerwick, 1997; Jiang et al., 2000).

Proposed biosynthetic pathways cannot be put forth for the non-volatile C20:5 oxylipins without making assumptions about the positions of the oxygenated functional groups. There are seven positional isomers of HpEPE in the known C20:5 oxylipin pathways of diatoms (Fig 1). The presence of a structural isomer of decatrienal $\mathrm{C}_{10} \mathrm{H}_{14} \mathrm{O}$ RT-4.9 and the putative NVO C10:2 1O RT-2.2 in the meta-lipidome suggests activity of 11(R)-lipoxygenase. However, it is impossible to conclude that the hydroperoxy and hydroxy groups of HpEPE and HEPE are located on the $11^{\text {th }}$ carbon with the information currently available. Based on the 0.87 minute offset in RT between C20:4 and C20:5 hydroxyacids and the retention times of 15-HpETE and 12-HpETE standards, the molecule annotated as HpEPE was more likely a 15-HpEPE than a 12HpEPE (Table 10). However, the small difference in retention time between positional isomers may not be easily resolved using theoretical retention times. For example, the 12 and 15- HpETE standards were only 0.22 minutes apart (Table 4). If only one isomer was present it would be difficult to confidently assign a positional level identification to the feature based on retention time alone.

Therefore, the best method of determining positional information would be fragmenting the molecules using multi-stage mass spectrometry. This would also allow for functional group level annotation of oxylipins that could not be resolved with authentic standards. All-IonFragmentation (AIF) was used in this study and was not selective enough to generate quality 
fragmentation information for the small oxylipins. However, future studies could employ the same streamlined lipidomic method presented here but paired with a selective fragmentation method. An example would be utilizing data dependent ms2 from a Thermo Q-Exactive Orbitrap MS to generate fragments for the most abundant ions which should produce high enough resolution $\mathrm{ms}^{2}$ spectra to determine the position of the oxygenated functional groups.

All other oxylipin studies mentioned in this chapter utilize both derivatization and fragmentation patterns to determine the functional groups and positional information of oxylipins. However, at least three different types of derivatization are necessary to measure the diversity of molecules observed in DYEatom dissolved lipidome (Cutignano et al., 2011).In addition to reducing time at the bench, the untargeted lipidomic method presented here is wellsuited to the structural diversity of oxylipins in the environment and allows for the discovery of novel compounds in the dissolved lipid pool. Expanding this method to include fragmentation will be crucial for future studies.

\subsubsection{Minor C20:5 components of the dissolved lipidome}

The majority of non-volatile oxylipins could not be annotated to the functional group level (88\%). Even within the well characterized C20:5 oxylipin class, there were two C20:5 2O isomers (RT-6.7 and 7.5), two C20:5 10 isomers (RT-5.9 and 6.8), three C20:5 30 isomers (RT1.7, 3.2, and 6.6), and two C20:5 $4 \mathrm{O}$ (RT-1.7 and RT- 3.2) isomers that could not be resolved. These molecules make up a minor fraction of the dissolved lipidome, $2.8 \%$ of the average peak area (data not shown), and all but the C20:5 20 isomers appear to be novel molecules in diatom oxylipin biosynthesis (Table 7).

The C20:5 20 isomers are hypothesized to be hydroxy epoxy acids (HepEPE), stemming from allene oxide synthase (AOS) activity (Fig 1). Authentic standards were not commercially available for hydroxy epoxy acids so I was not able to validate this putative annotation.

Theoretical retention times for several positional isomers of HepEPE were calculated from the octanol-water partition coefficients, suggesting much earlier elution of HepEPE around 4.8 minutes (Table 13). However, octanol water partition coefficients appear to inaccurately predict retention times of compounds with epoxy groups; the $\mathrm{RT}_{\log P}$ calculated for the epoxy acid C20:4 standard was 0.68 minutes earlier than observed. In the future, hydroxy epoxy acids could be 
isolated from Skeletonmena pseudocostatum, Skeletonmena marinoi, Leptocylindrus diatoms and/or Pseudeonitzchia delicatissimia and used as authentic standards (Barreiro et al., 2011; d'Ippolito et al., 2009; Fontana et al., 2007; Nanjappa et al., 2014).

The two C20:5 10 isomers at 5.9 and 6.8 minutes were enigmatic. The retention times were much later than any of the C20:4 standards would predict (Table 7), excluding the possibility of these molecules being keto-acids or epoxy-acids, the other known C20:5 10 oxylipins in diatoms. The $\mathrm{C} 20: 53 \mathrm{O}$ isomers could potentially be functional group analogs of Resolvin E1, a C20:5 trihydroxy acid that mediates human anti-inflammatory response, is involved in cell migration in the dermis and pulmonary arteries, and inhibits efflux transporters (Cholkar et al., 2015; Hiram et al., 2015; Kolanowski, 2015; Sawada et al., 2015). The two C20:5 4O isomers suggest sequential lipoxygenation of EPA by LOX to produce a dihydroperoxy acids. Such a mechanism is observed in Magnaporthe oryzae, a fungi that infects rice (Wennman et al., 2015). It is hypothesized that the fungi produces di-hydroperoxy acids from alpha-linolenic acid (C18:2) and other oxylipins as a strategy to oxidize the lipid membranes of rice cells and the protective plant cuticle during infection.

\subsection{Bioactivity of high abundance EPA derived non-volatile oxylipins}

The infochemical signals encoded by the abundant EPA oxylipins in the DYEatom metalipidome can be inferred from the bioactivities of these molecules in other systems. Culture studies with the diatom S. pseudocostaum found that HEPE, HepEPE, and keto-EPE decreased hatching success and induce apoptosis in the nauplii of the copepod Temora stylifera (Barreiro et al., 2011). In the northern Adriatic Sea, non-volatile oxylipins were more abundant than PUAs in the particulate phase and NVO concentrations were correlated with decreased hatching success of copepod eggs along the cruise track (Ianora et al., 2015). The dominance of nonvolatile oxylipins over PUAs in the potential oxylipin pool was consistent with our survey of the dissolved meta-lipidome which showed $47 \%$ of the total dissolve lipidome peak area was attributable to non-volatile oxylipin versus $0.01 \%$ for PUAs. In the northern Adriatic study, the NVO concentration was defined as the sum of the oxylipins described in culture and mesocosm extracts from S. marinoi, C. socialis, and C. affinis which included several structural isomers observed in the DYEatom cruise lipidome (Table 18; (Fontana et al., 2007; Gerecht et al., 2013; Gerecht et al., 2011). The relatively high concentrations of dissolved HpEPE and HEPE along 
the DYEatom cruise track indicate that non-volatile oxylipins may be influencing the reproductive success of copepods in the region which would ultimately impact the biological pump.

While copepods are not the major grazers of diatoms, like they were once thought to be, they consume $\sim 12 \%$ of global primary production (Calbet, 2001). Copepods and other grazers play a complicated role in the marine carbon cycle, disaggregating particles through sloppy feeding, exporting carbon to depth via fecal pellets, transporting carbon from the surface to depth via diel migrations (Ducklow et al., 2001). Many copepods are not deterred by oxylipins which means that there is a temporal de-coupling between the bioactivity of oxylipins and the biogeochemical repercussions (Barreiro et al., 2011; Leising et al., 2005). Feeding on oxylipin rich diatoms would result in a smaller population size for the next generation of copepods, decreasing the influence of zooplankton on the marine carbon cycle on annual timescales.

Microzooplankton grazing is a more important term for diatom mortality than copepod grazing, removing an average of $67 \%$ of daily phytoplankton growth in a review of 788 dilution experiments (Calbet, 2004). Unlike copepods, microzooplankton grazing behavior appears to be sensitive to oxylipins. When added to cultures, Leukotriene B4 and15-HpETE, were shown to interact with the diatom NO stress surveillance system and decrease microzooplankton grazing of P. tricornutum in a dose dependent fashion (Chapter 3). Leukotriene B4 and15-HpETE are C20:4 analogs to diHEPE and HpEPE which were abundant and varied significantly across the oxylipidome (Fig 7 and Fig 8A). In culture experiments described in Chapter 3, diHEPE (RT2.6) was among one of the oxylipins significantly upregulated when P. tricornutum was grazed by Oxhyrris marina. Additionally, minor C20:4 compounds ( $<0.2 \%$ of the total peak area), diHETE RT-3.7 and HpETE RT-5.9, are isomers of leukotriene B4 and 15-HpETE and were verified by authentic standards (Table 4B).

While 15-HpETE and Leukotriene B4 decreased microzooplankton grazing in Pt cultures, the bioactivity of the C20:5 structural analogs and C20:4 functional group analogs produced in situ would need to be tested independently. The extra double bond and potentially different positional configuration of the observed molecules compared to Leukotriene B4 and 15HpETE could confer a different bioactivity. For example, Resolvin E1 and Lipoxin A4/B4 are C20:5 and C20:4 trihydroxy acids that serve several distinct functions in human anti- 
inflammatory response. In conjunction, C20 NVOs have multiple signaling roles in the ocean, impacting both copepod egg viability and microzooplankton grazing. So the C20:5 oxylipins in situ could have effects of grazers, as well as, other organisms in the phycosphere. Towards this point, the C20:4 dihydroxy acids produced by red algae reduce epiphyte settlement and C18 dihydroxy acids are sex hormones in fungi (Serhan et al., 2009; Tsitsigiannis et al., 2004; Weinberger et al., 2011). Future amendment experiments aimed at determining the bioactivity and ecological importance of NVO should closely consider exactly which positional isomers are present in marine environments and the multiplicity of oxylipin signaling.

\subsection{Potentially bioactive C16 oxylipins}

The C16 oxylipin HpHME and HpHTrE made up more than $1 \%$ of the peak area in the meta-lipidome (Fig 7). 9S-HpHTrE is the precursor to octadienal (PUA C8:2) in T. rotula and bioactive in its own right, being implicated as one of many oxylipins that decreasing copepod hatching success in culture and in the environment (Barreiro et al., 2011; d'Ippolito et al., 2005; Ianora et al., 2015). HpHME is potentially the precursor to HHME another bioactive oxylipin observed in $S$. marinoi and $T$. rotula cultures and observed to decrease copepod hatching success (Fontana et al., 2007). C16:1 oxylipin pathways have not been described using isotopic labeling experiments as was done for C16:4, C16:3, C20:5, and C20:4 (Barofsky and Pohnert, 2007). However, d'Ippolito et al.(2005) concluded that the high stereospecificity of HHME produced by T. rotula suggested enzymatic production.

Following the typical enzymatic cascade of oxylipin biosynthesis in diatoms, HpHME would be produced by lipoxygenase and serve as the precursor to HHME production via peroxidase activity. But LOX enzymes are specific for the cis, cis-1,4 pentadienes of polyunsaturated fatty acids and are not thought to oxidize monounsaturated fatty acids. However, linoleate diol synthase (LDS) from fungi can convert C16:1 free fatty acid to HHME and dihydroxy HHME in culture experiments (Garscha et al., 2007a; Oliw et al., 2011). PpoA/8,11LDS enzyme in Aspergilus sp. creates hydroperoxy acids from18:2 fatty acids as an intermediate step in dihydroxy acid and hydroxy acid production (Andreou et al., 2009). Thus, LDS homologs may be responsible for HHME and HpHME production in diatoms. Lastly, many structural isomers of bioactive $\mathrm{C} 16$ compounds were observed in the data set but could not be annotated to 
the function group level (Table 19), making the prospect of integrating fragmentation into the lipidomic pipeline even more exciting.

\subsection{High abundance of a potentially novel algaecide in marine environments}

A compound putatively annotated as decatrienoic acid was the third most abundant compound in the dissolved meta-lipidome (6.6\% of total peak area) and does not fit into any known oxylipin pathways, diatom or otherwise. However, decatrienoic acid was isolated from Streptomyces viridochromogenes (Phylum Actinobacteria) and found to have herbicidal effects towards common duck weed (Lemna minor) and garden crest (Lepidium sativum) (Maier et al., 1999). Actinobacteria were the only bacterial subclade positively correlated with PUA concentrations in amendment experiments with sinking particles from the North Atlantic (Edwards et al., 2015). Therefore, decatrienoic acid may represent a bacterial contribution to the dissolved lipid pool potentially serving an algaecidal role in diatom-bacterial interactions.

Algaecides, that are not structurally related to oxylipins, are produced by roseobacteria living as epibionts of Emiliania huxleyi when their coccolithophore hosts begin to senesce (Seyedsayamdost et al., 2011). These roseobacticides elicit a switch from a mutualistic interaction between bacteria and phytoplankton into a parasitic interaction that is hypothesized to impact the marine carbon cycle by increasing the flow of carbon from the particulate pool to the dissolved pool. A similar interaction that is facilitated by oxylipins could exist between diatoms and their epibionts. Diatom associated bacterial strains in culture did exhibit remarkable tolerance to PUAs and natural particle associate bacteria increased their metabolic capacity in response to non-lethal doses of PUAs (Edwards et al., 2015; Ribalet et al., 2008).

Decatrienoic acid could also be a novel product of diatom oxylipin biosynthesis. Enzymatic cleavage of 11R-hydroperoxy eicosapentaenoic acid (C20:5 -2O) by hydroperoxy lyase (HPL) yields the PUA homolog of decatrienoic acid, decatrienal, and oxo-decadienoic acid (Fig 1). Oxylipin enzymes are known to have unique "catalytic plasticity"(Wasternack and Feussner, 2008). For example, in Arabidoposis, substitution of one critical amino acid in the allene oxide synthase (AOS) proteins results in an enzyme that functions as a HPL (Grechkin et al., 2008). And in tomatoes, the AOS enzyme functions both as an AOS and as an allene oxide cyclase (Toporkova et al., 2008). Extending the idea of catalytic plasticity, it is conceivable that 
a decatrienoic acid and decadienoic acid could be produced instead of hydroxy decadienoic acid upon cleavage of 11-HPETE by a sloppy/multifunctional HPL enzyme (Figure 11). There was HPL activity in the water column with two molecular species annotated as PUAs (Table 11).

The proposed biosynthetic pathway could also be utilized by marine bacteria. In fact, decadienoic acid was also produced by Streptomyces viridiochromogenes and demonstrated the same algaecidal activity as decatrienoic acid (Maier et al., 1999). Future studies using bacterial and diatom isolates would need to be conducted to determine the origin and biosynthesis of decatrienoic acid. Regardless, the abundance of decatrienoic acid in the environment and its potential bioactivity is intriguing and supports the hypothesis that novel infochemical signaling is occurring in diatom blooms.

\subsection{Shifts in the dissolved lipidome across the cruise transect driven by fluctuations in major free fatty acids and oxylipins and tied to viral lysis}

Principal component analysis was used to give ecological context to the variability in the DYEatom cruise dissolved lipidome. Sixteen of the nineteen compounds significantly loaded on principal component 1 (PC1) were also dominant molecular species in the dissolved metaoxylipidome (Fig 8A; Fig 7). Two of those species, HEPE and HpEPE were structural isomers of known oxylipin signaling compounds (Section 4.2). PC1 carried 83\% of the inertia of the data and reflected the inverse relationship between indicators of diatom biomass degradation, phaeophytin and bSi, and indicators of healthy phytoplankton blooms, chlorophyll and dissolved inorganic nutrients (Fig 8B). Cosine squared values of the supplemental variables indicate that PC1 contributed the most to the loading of phaeophytin (Table 14C). Phaeophytin is a degradation product of chlorophyll that is a biomarker for both phytoplankton predation and senescence, the molecular level shut down of the cell as it dies and is exported to depth (Killops and KIllops, 2005). Thus, variations in abundant, bioactive molecular species were strongly correlation with diatom demise across the DYEatom cruise, corroborating the hypothesis that oxylipin infochemical signaling impacts the fate of organic carbon.

Since, covariance PCA is weighted towards the molecules with the largest variance, level scaling was applied to assess more subtle changes in the dissolved lipidome. By looking at the 
abundances of a molecular species with respect to the mean abundances of those molecules across the dataset, major and minor compounds were given equal weight in the subsequent analyses (Fig 9B). Similarity profile analysis revealed that the eleven dissolved lipidomes clustered into six distinct groups that did not separate strictly according to station or depth (Fig 9A). For example, samples from the 55\% Io depth and the DCM at St 8 and St 4 clustering separately from one another, demonstrating that there can be significant shifts in the lipidome throughout the water column at one discrete location. However, this is not always the case; the two depths sampled at St 6 were statistically similar. These results indicate infochemical signaling varies spatial-temporally and across depth horizons, suggesting that microbial interactions are likely dramatically different in parcels of water only a few meters apart.

In addition to being the biological variable underlying the large variations in the metalipidome, phaeophytin was most attributable to similarities in dissolved lipidome structure across samples that were abundance-independent (Table 17; PERMANOVA p-value <0.1). However, phaeophytin only accounted for $15 \%$ of the variability in the data. Phaeophytin is a degradation product of chlorophyll and similar to oxylipin abundance it could be associated with processes that damage the cell such as, grazing, lytic viral infection, and nutrient stress. Of these processes, RNA viral reads were most significantly correlated with phaeophytin concentrations across the dissolved lipidome samples $(\mathrm{F}=0.01$; Table 19$)$. There was also a slightly less significant relationship between phaeophytin concentration and grazing $(\mathrm{F}=0.02)$. While viral infection was not directly measured, the abundance of RNA viral reads is considered a measurement of cell associated RNA viruses since they were determined by metatranscriptomic analysis of sea water filtered through $1.2 \mu \mathrm{m}$. These cell-associated viruses may be living inside infected cells but they could also be free in the water column adsorbing to cells during filtration. In the latter case, the viruses may represent a recent viral lysis event. Re-evaluating the lipidomic similarities between samples in light of processes associated with phaeophytin production, it became apparent that the samples from the stations with the highest total RNA viral reads throughout the water column, St7 and St10, formed a distinct cluster (Group III) with the highest abundance and richness of FFA and oxylipins (Fig 4F; Fig 9A; Fig 6A \& C).

Total RNA viral reads were not measured at every depth therefore PC loadings could not be generated for this variable. However, the PC1 loading scores of the samples were significantly 
and negatively correlated with total RNA viral reads, showing a better and more significant correlation than phaeophytin, the core supplemental variable best described by PC1 (Table 15, Table 14C). St7 had the highest total viral reads of any station across the cruise track (Fig 4F). However, we do not have viral data for the depth at which the lipidome was sampled at St7. As a consequence, one of the two high oxylipin, high viral abundance samples was not included in the regression of PC1 against total viral reads. The fact that the relationship between dissolved lipidomic structure and RNA viral reads was apparent without considering one of the potentially highest viral samples act as an accidental Monte Carlo test and strengthened our confidence in this finding.

Viral ecology of eukaryotes in the ocean is a burgeoning field unto itself. For the last three decades, the focus of had been on DNA viruses which tend to be bacteriophages and, like their hosts, dominate the marine environment. A 2013 paper by Stewart and Culley asserted that RNA viruses can be equally as abundant as DNA viruses in the marine viriome. Eukaryotes are thought to be the primary hosts of RNA viruses in the ocean. And thus, diatom virus diversity has been under sampled and the importance of RNA viral infection is only beginning to be understood. To date, six single stranded RNA diatom viruses have been isolated from the hosts: Rhizosolenia setigera, Chaetoceros tenuissimus, Chaetoceros socialis, Chaetoceros sp., and a Pennate diatom (Kimura and Tomarua, 2015; Nagasaki et al., 2004; Shirai et al., 2008; Tomaru et al., 2009; Tomaru et al., 2012; Tomaru et al., 2013).

Sequences homologous to Phytopthora infestans RNA virus 1 (PiRV-1) were generally the most abundant RNA viral reads across the DYEatom cruise (Fig 4G). Sequences with homology to know diatom viruses, Chaetoceros. sp RNA Virus 2 and Marine RNA virus JP-A, only dominated the viral meta-transcriptome when total viral reads were low. PiRV- 1 infects an oomycete within the stramenopile family that is responsible for potato blight (Cai et al., 2009). The most comprehensive assessment of RNA viral assemblages in the ocean to date did not find homologous sequences to PiRV-1 amongst the sixty-four RNA-dependent RNA polymerases (RdRp) regions translated from Kaneohe Bay in 2009 and 2010 (Culley et al., 2014). RdRp genes are highly conserved regions of the genome that are diagnostic for RNA viruses. All of the metatranscriptomic reads sequenced from the DYEatom cruise that had homology with the PiRV-1genenome were annotated as hypothetical proteins (Table 2). There were no hits to PiRV- 
1 RdRp. Since RNA viruses tend to be very host specific, the hits to the PiRV-1 genome likely represent conserved regions in the genomes of viruses that have evolved to infect stramenopiles, not the presence of PiRV-1 in marine ecosystems. However, the PiRV hypothetical proteins were not observed in the global ocean sampling study core cluster and may represent previously undescribed viruses in the marine environment (Table 2).

\subsection{Grazing and Nutrient Stress}

Grazing and nutrient stress were not strongly linked to the dissolved lipidomic structure in the California Coastal System. Grazing measurements were highest at St 1, 2, and 8 with rates above 1 day $^{-1}$, whereas, St 4, 6, 7, and 10 had much lower rates (Fig 5A). There were only four depths at which both grazing was measured and the dissolve lipidome was sampled. Grazing was not correlated with PCA loadings of those samples (Table 15) but the small sample size could have led to a type II error. The dissolved lipidome from the sample with the highest grazing rates (St 8-1m) clustered adjacent to but separate from the high oxylipin, high viral abundance Group III, sharing only $20 \%$ similarity in relative abundance of molecular species and exhibiting a lower abundance and richness of oxylipins (Fig 9A, Fig 9B, Fig 6A \& C). Grazing was correlated with phaeophytin across the cruise track (Table 19).Thus microzooplankton grazing may be associated with some upregulation of oxylipins but not to the degree that viruses are.

There is some statistical evidence to suggest that nutrient stress may influence the structure of the dissolve lipidome but it is not conclusive. PC2 captured $9.38 \%$ of the variation of the meta-lipidome and it was most important to the loading of the $\mathrm{dSi}$ (cosine ${ }^{2}$ values Table 14C). PC2 also separated the nineteen significantly varying molecular species based on whether they were a free fatty acid or oxylipins (Fig 8A). Oxylipins loaded opposite of dSi suggesting higher production of these oxylipins when dSi concentrations were low. This is consistent with mesocosm and culture experiments showing the high levels of PUA production under Si stress compared to P and N stress (Ribalet et al., 2009; Vidoudez et al., 2011b). However, the regression of PC2 loadings with dSi concentrations across the samples was not significant and PC2 captures a small percentage of the variability in the data (Table12; Fig 8). Furthermore, Sistress was specifically measured across the cruise track and did not correlate with the principal components (Table 15). 
There is more evidence that PC2 described some aspect of nutrient stress. When regressed against PC2, N:P ratios were significantly correlated (Table 15). N:P ratios were also correlated with potential PUA concentrations across seven biogeographical provinces in the N. Atlantic (Bartual and al, 2014). N:P ratios below Redfield (16:1) can indicate nitrogen stress which has been shown to decrease PUA potential in Skeletonema cultures but increase PUA potential in Skeletonema mesocosms (Ribalet et al., 2009; Vidoudez et al., 2011b). High N:P ratios can indicate phosphorous stress which was shown to increase PUA production upon sonication in both mesocosm and culture experiments but the California Coastal System is considered nitrogen limited not phosphorus limited (Moore et al., 2002). Therefore, the positive loading of N:P on PC2 with the oxylipins would suggest that the significantly varying nonvolatile oxylipins were anti-correlated with nitrogen stress. However, multivariate analysis that weighted abundant and minor compounds equally did not attribute any of the nutrient state variables to the similarities in meta-lipidome structure, only phaeophytin (Table 17).

Considering the strong correlation between phaeophytin and dissolve lipidome variability and structure across the DYEatom cruise, it is speculated that the physical mechanisms (grazing and viral lysis) that release oxylipins and produce phaeophytin have a larger impact on the dissolved oxylipin pool in situ than the physiological mechanisms that control potential oxylipin concentrations (i.e. nutrient stress). Had we investigated the potential oxylipin concentrations across the cruise track by simulating grazing/viral lysis through sonication, we may have seen stronger links to nutrient stress.

\subsection{Virally induced infochemical signaling: balancing mechanisms of diatom mortality}

Throughout the marine literature, oxylipin production has been studied in the context of grazing. However, the lipidomic analysis presented here changes that paradigm and links oxylipin production to RNA viruses. A new infochemical signaling role for oxylipins is proposed that allows diatoms to balance mechanisms of mortality in the upper ocean, deterring microzooplankton grazing while limiting viral encounter rates. 


\subsubsection{Increased oxylipin production associated with RNA viruses decreases microzooplankton grazing}

Low grazing rates were observed at St 7 and 10, where viruses dominated and oxylipin abundances were high, supporting the hypothesis that oxylipin production deters microzooplankton grazing. In addition to the abundant non-volatile oxylipins identified using PCA, significant shifts were observed in the less abundant, small molecular species when considering relative changes across the dissolved lipidome (Figure 9). The relative abundances of $\mathrm{C}_{10} \mathrm{H}_{14} \mathrm{O}$ RT-4.9 and dodecatrienal RT-4.9 were significantly different from other molecules across the DYEatom cruise, clustering together in Group 37 (Table 16; Fig 9C). And these molecular species were only detectable at the high viral count stations (Fig 9B). The small unknown molecule $\mathrm{C}_{10} \mathrm{H}_{14} \mathrm{O}$ RT-4.9 is a structural isomer of decatrienal, a PUA associated with teratogenic effects in copepod larvae (Miralto et al., 1999). Dodecatrienal is a C12 PUA that has not previously been described in phytoplankton. The C10 PUA, decadienal, was shown to decrease microzooplankton grazing in Chapter 3 and dodecatrienal may serve a similar role. However, the homologous C12:2 PUA, dodecadienal, was identified as a sex pheromone in the western tent caterpillar (Chisholm et al., 1981; Underhill et al., 1980). The homologous PUA acid, 12-oxo-dodecatrienoic acid is produced during the biosynthesis of fucoserratene, a pheromones in brown algae (Pohnert and Boland, 2002) and other oxylipin molecules have been identified as sex pheromones in brown algae, fresh water diatoms, and the model diatom Phaeodactylum tricornutum (Hombeck and Boland, 1998; Pohnert and Boland, 1996; Wendel and Jüttner, 1996). Dodecadienal was also recently observed in the Kenya tree coral, Capnella imbricate and hypothesized to function in wound response (Teder et al., 2015). Given that dodecatrienal is homology to known infochemical signals in other systems, it is likely bioactive and functioning as an infochemical signal in these high oxylipin, high viral abundance stations.

Lastly, two C20:5 non-volatile oxylipins were observed in the dissolved lipidome that were structurally analogous to known C20:4 NVO microzooplankton deterrents and two C20:4 NVO were observed that were functional group analogs (Section 4.2). Deterrence of microzooplankton grazers by oxylipins could account for the lack of correlation between microzooplankton grazing and the dissolved meta-lipidome, as any oxylipins produced by 
microzooplankton grazers would lower the grazing rate in the region and prevent additional oxylipin production from grazing.

If this interpretation of the data is correct, having some level of viral infection within a population would benefit the phytoplankton community by decreasing grazing pressure on the community as a whole. Such a mechanism would certainly be evolutionarily beneficial to viruses, allowing the host population to thrive and not succumb to grazing before they could be infected. Supporting this hypothesis, previous work by Suttle and Feng (1992) and Gonzalez and Suttle (1993), found that flagellates ingested viruses a very low rates. Whereas, grazing by copepods is an important mechanism for the removal of phytoplankton viruses from the water column and export to depth (Frada et al., 2014), presumably because they are not deterred by the oxylipins produced by virally infected cells.

4.7.2 Increased oxylipin abundance associated with viruses may limit viral infection by removal of viruses from the upper ocean via aggregate formation and export.

Virally induced oxylipin infochemical signaling would benefit diatoms by reducing one source of mortality, microzooplankton grazing, in the face of another, viral infection. A feedback loop within virally induced oxylipin infochemical signaling may actually limit viral infection, as well. Removing virally infected cells from the water column by sedimentation has been proposed as an effective strategy to ameliorate the threat of demise by viral lysis (Mojica and Brussaard, 2014). PUAs have been shown to decrease motility and increase biofilm formation in benthic diatoms (Leflaive and Ten-Hage, 2011). In conjunction, experiments with the Phaeocystis globosa-virus system have shown that hours before host cells lyse they exude organic compounds that lead to the attachment of Alteromonas bacteria to infected cells, aggregation of cells, and increased substrate assimilation (Sheik et al., 2014). Phaeocystis is among the nondiatom eukaryotic phytoplankton that produce oxylipins. Therefore, viral induction of infochemical signaling between marine phytoplankton and the microbial community could influence carbon export by inducing diatom aggregation.

We propose a scheme by which viral infection of diatoms resulted in a release of oxylipins into the dissolved pool stimulating aggregation and export of infected diatom cells 
from the densely populated upper waters before viral lysis and larger scale infection could occur (Figure 12). At the same time this process deters grazing and stimulates diatom associated bacteria. This type of infochemical signaling could function to prolong diatom blooms by dampening diatom mortality terms and promoting the recycling of nutrients.

Preliminary results from cultures show that many of the same oxylipin pathways utilized by phytoplankton along the DYEatom cruise dissolved lipidome may be up-regulated intracellularly after exposure of Chaetoceros tenuissimus to the RNA virus, CtenRNAV (Appendix E). To test the sedimentation aspect of the hypothesis, aggregation, the dissolved lipidome, and settling velocities of cells could be measured throughout viral infection. Ideally, these studies would be conducted in co-culture with diatom epibionts as they play an important role in biofilm formation and aggregate in P. globosa (Sheik et al., 2014). Alternatively, molecules isolated from the dissolved lipidome of infected cells could be added to natural diatom assemblages to test for induction of TEP. To test the grazing aspect of this hypothesis, ingestion rates could be compared between treatments where microzooplankton were fed uninfected cells and treatments where microzooplankton were fed infected cells. Or microzooplankton grazing rates could be measured in the presence of molecules that are unique to the dissolve lipidome of infected cells compared to uninfected cells. Testing this hypothesis in the environment would require a rigorous field campaign that tracks a diatom bloom where viral infection is occurring through the course of its life cycle, sampling the dissolved lipidome, POC export dynamics, absorption of viruses to sinking particles, bacterial community structure and metabolism, as well as, grazing rates. A similarly rigorous field campaign was executed on the NA-VICE cruise presented in Chapter 2 and can be successful with proper preparation and effective collaboration.

\section{Conclusion}

Lipidomic analysis proved to be an excellent tool to assess the diversity of non-volatile oxylipins in the marine environment. Diatom derived free fatty acids and EPA oxylipins were the most abundant molecules in the dissolved lipidome across the DYEatom cruise track and changes in the abundances of these molecules were linked to the presence of phaeophytin and viruses. Two of the four significant C20:5 derived oxylipins observed, HEPE and HpEPE, are 
functional group isomers of compounds known to decrease copepod reproductive success in culture studies and in the field. Culture studies in Chapter 3 with C20:4 analogs of compounds observed in the study, HpEPE and diHEPE, suggest that C20:5 derived oxylipins also play a role in deterring microzooplankton grazing. In conjunction, the stations with the lowest microzooplankton grazing rates had the largest dissolved lipidomes and the highest abundance of dodecatrienal, a small molecule belonging to the PUA subclass of oxylipins which were previously implicated as microzooplankton deterrents, mediators of organic matter respiration by particle associated bacteria, and detrimental to copepod hatching success (Edwards et al., 2015; Johnson et al., submitted; Miralto et al., 1999). Nutrient stress and grazing are typically thought to influence oxylipin potential in culture and mesocosms but mechanical disruption of the cells via viral lysis appeared to have a larger influence on the dissolved lipidome in situ.

This study is the first to investigate oxylipins in the context of viral infection, revealing new avenues for infochemical signaling to impact the flow of carbon through the microbial loop. In addition to acting as a chemical defense against grazing, oxylipins may remove viruses from the water column by stimulating aggregation and export of infected cells to depth. Aggregation of cells and the bioactivity of oxylipins on diatom epibionts would also promote nutrient recycling. Therefore, virally induced infochemical signaling may play a role in mitigating all mechanisms of diatom demise and prolonging diatom blooms. Field and culture studies should be employed to test this hypothesis. Lastly, this study is one of the first forays into -omic era analysis of signaling molecules in the environment, revealing many oxylipin compounds that are novel in the marine environment. Future meta-lipidomic studies that build upon this pipeline will undoubtedly advance our understanding of oxylipins present in the environment and the ecological relevance of their bioactivity.

References

Abdi, H.a.W.L.J., 2010. Principal component analysis. Wiley Interdisciplinary Reviews: Computational Statistics 2 , 433--459.

Anderson, M.J., 2001. A new method for non-parametric multivariate analysis of variance. Austral Ecology 26, 3246.

Andreou, A., Brodhun, F., Feussner, I., 2009. Biosynthesis of oxylipins in non-mammals. Progress in Lipid Research 48, 148-170. 
Barofsky, A., Pohnert, G., 2007. Biosynthesis of polyunsaturated short chain aldehydes in the diatom Thalassiosira rotula. Organic Letters 9, 1017-1020.

Barreiro, A., Carotenuto, Y., Lamari, N., Esposito, F., D'Ippolito, G., Fontana, A., Romano, G., Ianora, A., Miralto, A., Guisande, C., 2011. Diatom induction of reproductive failure in copepods: The effect of PUAs versus non volatile oxylipins. Journal of Experimental Marine Biology and Ecology 401, 13-19.

Bartual, A., al, e., 2014. Polyunsaturated Aldehydes from Large Phytoplankton of the Atlantic Ocean Surface (42 N to $33 \mathrm{~S})$. Marine drugs 12, 682-699.

Cai, G., Fry, W.E., Hillman, B.I., Myers, K., 2009. An RNA virus from Phytophthora infestans with no apparent similarity to known viruses. Phytopathology 99, S18-S18.

Calbet, A., 2001. Mesozooplankton grazing effect on primary production: A global comparative analysis in marine ecosystems. Limnology and Oceanography 46, 1824-1830.

Calbet, A, Landry, M., 2004. Phytoplankton growth, microzooplankton grazing, and carbon cycling in marine systems. Limnology and Oceanography 49, 51--57.

Chiang, N., Arita, M., Serhan, C.N., 2005. Anti-inflammatory circuitry: Lipoxin, aspirin-triggered lipoxins and their receptor ALX. Prostaglandins Leukotrienes and Essential Fatty Acids 73, 163-177.

Chisholm, M.D., Steck, W.F., Bailey, B.K., Underbill, E.W., 1981. Synthesis of sex pheromone components of the forest tent caterpillar,Malacosoma disstria (Hübner) and of the western tent caterpillar,Malacosoma californicum (Packard). Journal of Chemical Ecology 7, 159-164.

Cholkar, K., Trinh, H.M., Vadlapudi, A.D., Wang, Z.Y., Pal, D., Mitra, A.K., 2015. Interaction Studies of Resolvin E1 Analog (RX-10045) with Efflux Transporters. Journal of Ocular Pharmacology and Therapeutics 31, 248-255.

Collins, J., Edwards, B., Fredricks, H., Van Mooy, B., in prep. A rules-based lipidomics pipeline for discovery of oxidative stress biomarkers in large datasets.

Culley, A.I., Mueller, J.A., Belcaid, M., Wood-Charlson, E.M., Poisson, G., Steward, G.F., 2014. The Characterization of RNA Viruses in Tropical Seawater Using Targeted PCR and Metagenomics. Mbio 5.

Cutignano, A., Lamari, N., d'Ippolito, G., Manzo, E., Cimino, G., Fontana, A., 2011. Lipoxygenase products in marine diatoms: A concise analytical method to explore the functional potential of oxylipins. Journal of Phycology 47, 233-243.

d'Ippolito, G., Cutignano, A., Briante, R., Febbraio, F., Cimino, G., Fontana, A., 2005. New C16 fatty-acid-based oxylipin pathway in the marine diatom Thalassiosira rotula. Org Biomol Chem 3, 4065-4070.

d'Ippolito, G., Lamari, N., Montresor, M., Romano, G., Cutignano, A., Gerecht, A., Cimino, G., Fontana, A., 2009. 15S-Lipoxygenase metabolism in the marine diatom Pseudo-nitzschia delicatissima. New Phytologist 183, 10641071.

Ducklow, H.W., Steinberg, D.K., Buesseler, K.O., 2001. Upper ocean carbon export and the biological pump. Oceanography 14, 50-58.

Dunstan, G.A., Volkman, J.K., Barrett, S.M., Leroi, J.M., Jeffrey, S.W., 1994. Essential polyunsaturated fatty acids from 14 species of diatom (Bacillariophyceae). Phytochemistry 35, 155-161.

Edwards, B.R., Bidle, K.D., Van Mooy, B.A.S., 2015. Dose-dependent regulation of microbial activity on sinking particles by polyunsaturated aldehydes: Implications for the carbon cycle. Proceedings of the National Academy of Sciences 112, 5909-5914. 
Feussner, I., Wasternack, C., 2002. The lipoxygenase pathway. Annual Review of Plant Biology 53, 275-297.

Fontana, A., d'Ippolito, G., Cutignano, A., Romano, G., Lamari, N., Gallucci, A.M., Cimino, G., Miralto, A., Ianora, A., 2007. LOX-induced lipid peroxidation mechanism responsible for the detrimental effect of marine diatoms on Zooplankton grazers. Chembiochem 8, 1810-1818.

Frada, M.J., Schatz, D., Farstey, V., Ossolinski, J.E., Sabanay, H., Ben-Dor, S., Koren, I., Vardi, A., 2014. Zooplankton May Serve as Transmission Vectors for Viruses Infecting Algal Blooms in the Ocean. Current Biology 24, 2592-2597.

Garscha, U., Jerneren, F., Chung, D., Keller, N.P., Hamberg, M., Oliw, E.H., 2007a. Identification of dioxygenases required for aspergillus development - Studies of products, stereochemistry, and the reaction mechanism. Journal of Biological Chemistry 282, 34707-34718.

Garscha, U., Jerneren, F., Chung, D., Keller, N.P., Hamberg, M., Oliw, E.H., 2007b. Identification of dioxygenases required for Aspergillus development. Studies of products, stereochemistry, and the reaction mechanism. J Biol Chem 282, 34707-34718.

Gerecht, A., Carotenuto, Y., Ianora, A., Romano, G., Fontana, A., d'Ippolito, G., Jakobsen, H.H., Nejstgaard, J.C., 2013. Oxylipin production during a mesocosm bloom of Skeletonema marinoi. Journal of Experimental Marine Biology and Ecology 446, 159-165.

Gerecht, A., Romano, G., Ianora, A., d'Ippolito, G., Cutignano, A., Fontana, A., 2011. Plasticity of oxylipin metabolism among clones of the marine diatom Skeletonema marinoi (Bacillariophyceae). Journal of Phycology 47, 1050-1056.

Gonzalez, J.M., Suttle, C.A., 1993. Grazing by marine nanoflagellates on viruses and virus-sized particles- ingestion and digestion. Marine Ecology Progress Series 94, 1-10.

Grechkin, A.N., Mukhtarova, L.S., Latypova, L.R., Gogolev, Y., Toporkova, Y.Y., Hamberg, M., 2008. Tomato CYP74C3 is a Multifunctional Enzyme not only Synthesizing Allene Oxide but also Catalyzing its Hydrolysis and Cyclization. Chembiochem 9, 2498-2505.

Hiram, R., Rizcallah, E., Marouan, S., Sirois, C., Sirois, M., Morin, C., Fortin, S., Rousseau, E., 2015. Resolvin E-1 normalizes contractility, $\mathrm{Ca} 2+$ sensitivity and smooth muscle cell migration rate in TNF-alpha- and IL-6-pretreated human pulmonary arteries. American Journal of Physiology-Lung Cellular and Molecular Physiology 309, L776L788.

Hombeck, M., Boland, W., 1998. Biosynthesis of the algal pheromone fucoserratene by the freshwater diatom Asterionella formosa (Bacillariophyceae). Tetrahedron 54, 11033-11042.

Hoppe, H.G., 1993. Use of fluorogenic model substrates for extracellular enzyme activity (EEA) measurement of bacteria. Handbook of methods in aquatic microbial ecology, 423-431.

Hummel, J., Segu, S., Li, Y., Irgang, S., Jueppner, J., Giavalisco, P., 2011. Ultra performance liquid chromatography and high resolution mass spectrometry for the analysis of plant lipids. Frontiers in Plant Science 2.

Ianora, A., Bastianini, M., Carotenuto, Y., Casotti, R., Roncalli, V., Miralto, A., Romano, G., Gerecht, A., Fontana, A., Turner, J.T., 2015. Non-volatile oxylipins can render some diatom blooms more toxic for copepod reproduction. Harmful Algae 44, 1-7.

Jiang, Z.D., Gerwick, W.H., 1997. Novel oxylipins from the temperate red alga Polyneura latissima: Evidence for an arachidonate 9(S)-lipoxygenase. Lipids 32, 231-235. 
Jiang, Z.D., Ketchum, S.O., Gerwick, W.H., 2000. 5-Lipoxygenase-derived oxylipins from the red alga Rhodymenia pertusa. Phytochemistry 53, 129-133.

Johnson, M., Edwards, B., Beaudoin, D., Van Mooy, B.A.S., Vardi, A., submitted. Oxylipins mediate microzooplankton grazing on diatoms.

Killops, S., KIllops, V., 2005. Diagenesis at the molecular level, Introduction to Organic Chemistry. Blackwell Publishing, Oxford, UK, pp. 174-195.

Kimura, K., Tomarua, Y., 2015. Discovery of Two Novel Viruses Expands the Diversity of Single-Stranded DNA and Single-Stranded RNA Viruses Infecting a Cosmopolitan Marine Diatom. Applied and Environmental Microbiology 81, 1120-1131.

Kolanowski, W., 2015. Role of omega-3 polyunsaturated fatty acids in the course of inflammation. Medycyna Weterynaryjna-Veterinary Medicine-Science and Practice 71, 608-614.

Krause, J.W., Brzezinski, M.A., Jones, J.L., 2011. Application of low-level beta counting of Si-32 for the measurement of silica production rates in aquatic environments. Marine Chemistry 127, 40-47.

Landry, M.R., Hassett, R.P., 1982. Estimating the grazing impact of marine micro-zooplankton. Marine Biology 67, 283-288.

Lavrentyev, P., Franzè, G., Pierson, J., Stoecker, D., 2015. The Effect of Dissolved Polyunsaturated Aldehydes on Microzooplankton Growth Rates in the Chesapeake Bay and Atlantic Coastal Waters. Marine Drugs 13, 2834.

Leflaive, J., Ten-Hage, L., 2011. Effects of 2E,4E-decadienal on motility and aggregation of diatoms and on biofilm formation. Microb Ecol 61, 363-373.

Leising, A.W., Pierson, J.J., Halsband-Lenk, C., Horner, R., Postel, J., 2005. Copepod grazing during spring blooms: Does Calanus pacificus avoid harmful diatoms? Progress in Oceanography 67, 384-405.

Levy, B.D., Romano, M., Chapman, H.A., Reilly, J.J., Drazen, J., Serhan, C.N., 1993. Human alveolar macrophages have 15-lipoxygenase and generate 15(s)-hydroxy-5,8,11-cis-13trans-eicosatetraenoic acid and lipoxins. Journal of Clinical Investigation 92, 1572-1579.

Maier, A., Muller, J., Schneider, P., Fiedler, H.P., Groth, I., Tayman, F.S.K., Teltschik, F., Gunther, C., Bringmann, G., 1999. (2E,4Z)-decadienoic acid and (2E,4Z,7Z)-decatrienoic acid, two herbicidal metabolites from Streptomyces viridochromogenes Tu 6105. Pesticide Science 55, 733-739.

Marchetti, A., Schruth, D.M., Durkin, C.A., Parker, M.S., Kodner, R.B., Berthiaume, C.T., Morales, R., Allen, A.E., Armbrust, E.V., 2012. Comparative metatranscriptomics identifies molecular bases for the physiological responses of phytoplankton to varying iron availability. Proceedings of the National Academy of Sciences of the United States of America 109, E317-E325.

Melamud, E., Vastag, L., Rabinowitz, J.D., 2010. Metabolomic analysis and visualization engine for LC,àí MS data. Analytical chemistry 82, 9818-9826.

Miralto, A., Barone, G., Romano, G., Poulet, S.A., Ianora, A., Russo, G.L., Buttino, I., Mazzarella, G., Laabir, M., Cabrini, M., 1999. The insidious effect of diatoms on copepod reproduction. Nature 402, 173-176.

Mitchell, S., Thomas, G., Harvey, K., Cottell, D., Reville, K., Berlasconi, G., Petasis, N.A., Erwig, L., Rees, A.J., Savill, J., Brady, H.R., Godson, C., 2002. Lipoxins, aspirin-triggered epi-lipoxins, lipoxin stable analogues, and the resolution of inflammation: Stimulation of macrophage phagocytosis of apoptotic neutrophils in vivo. Journal of the American Society of Nephrology 13, 2497-2507. 
Mojica, K.D.A., Brussaard, C.P.D., 2014. Factors affecting virus dynamics and microbial host-virus interactions in marine environments. Fems Microbiology Ecology 89, 495-515.

Moore, J.K., Doney, S.C., Glover, D.M., Fung, I.Y., 2002. Iron cycling and nutrient-limitation patterns in surface waters of the World Ocean. Deep-Sea Research Part Ii-Topical Studies in Oceanography 49, 463-507.

Nagasaki, K., Tomaru, Y., Katanozaka, N., Shirai, Y., Nishida, K., Itakura, S., Yamaguchi, M., 2004. Isolation and characterization of a novel single-stranded RNA virus infecting the bloom-forming diatom Rhizosolenia setigera. Applied and Environmental Microbiology 70, 704-711.

Nanjappa, D., Ippolito, G., Gallo, C., Zingone, A., Fontana, A., 2014. Oxylipin Diversity in the Diatom Family Leptocylindraceae Reveals DHA Derivatives in Marine Diatoms. Marine Drugs 12, 368.

Oliw, E.H., Wennman, A., Hoffmann, I., Garscha, U., Hamberg, M., Jerneren, F., 2011. Stereoselective oxidation of regioisomeric octadecenoic acids by fatty acid dioxygenases. Journal of Lipid Research 52, 1995-2004.

Pacheco, L., Uribe, E., Pino, J., Troncoso, J., Quiróz, A., 2014. The Effect of UV Light and $\mathrm{CO}_{2}$ in the Production of Polyunsaturated Aldehydes in Skeletonema costatum (Bacillariophycea). American Journal of Plant Science 5, 3632-3641.

Papayianni, A., Serhan, C.N., Brady, H.R., 1996. Lipoxin A(4) and B-4 inhibit leukotriene-stimulated interactions of human neutrophils and endothelial cells. Journal of Immunology 156, 2264-2272.

Pohnert, G., Boland, W., 1996. Biosynthesis of the algal pheromone hormosirene by the fresh-water diatom Gomphonema parvulum (Bacillariophyceae). Tetrahedron 52, 10073-10082.

Ribalet, F., Bastianini, M., Vidoudez, C., Acri, F., Berges, J., Ianora, A., Miralto, A., Pohnert, G., Romano, G., Wichard, T., Casotti, R., 2014. Phytoplankton Cell Lysis Associated with Polyunsaturated Aldehyde Release in the Northern Adriatic Sea. PLoS ONE 9, e85947.

Ribalet, F., Berges, J.A., Ianora, A., Casotti, R., 2007a. Growth inhibition of cultured marine phytoplankton by toxic algal-derived polyunsaturated aldehydes. Aquatic Toxicology 85, 219-227.

Ribalet, F., Intertaglia, L., Lebaron, P., Casotti, R., 2008. Differential effect of three polyunsaturated aldehydes on marine bacterial isolates. Aquatic Toxicology 86, 249-255.

Ribalet, F., Vidoudez, C., Cassin, D., Pohnert, G., Ianora, A., Miralto, A., Casotti, R., 2009. High plasticity in the production of diatom-derived polyunsaturated aldehydes under nutrient limitation: physiological and ecological implications. Protist 160, 444-451.

Ribalet, F., Wichard, T., Pohnert, G., Ianora, A., Miralto, A., Casotti, R., 2007b. Age and nutrient limitation enhance polyunsaturated aldehyde production in marine diatoms. Phytochemistry 68, 2059-2067.

Sawada, Y., Honda, T., Hanakawa, S., Nakamizo, S., Murata, T., Ueharaguchi-Tanada, Y., Ono, S., Amano, W., Nakajima, S., Egawa, G., Tanizaki, H., Otsuka, A., Kitoh, A., Dainichi, T., Ogawa, N., Kobayashi, Y., Yokomizo, T., Arita, M., Nakamura, M., Miyachi, Y., Kabashima, K., 2015. Resolvin E1 inhibits dendritic cell migration in the skin and attenuates contact hypersensitivity responses. The Journal of experimental medicine 212, 1921-1930.

Serhan, C.N., Yang, R., Martinod, K., Kasuga, K., Pillai, P.S., Porter, T.F., Oh, S.F., Spite, M., 2009. Maresins: novel macrophage mediators with potent antiinflammatory and proresolving actions. J Exp Med 206, 15-23.

Seyedsayamdost, M.R., Carr, G., Kolter, R., Clardy, J., 2011. Roseobacticides: Small Molecule Modulators of an Algal-Bacterial Symbiosis. Journal of the American Chemical Society 133, 18343-18349. 
Sheik, A.R., Brussaard, C.P.D., Lavik, G., Lam, P., Musat, N., Krupke, A., Littmann, S., Strous, M., Kuypers, M.M.M., 2014. Responses of the coastal bacterial community to viral infection of the algae Phaeocystis globosa. Isme Journal 8, 212-225.

Shirai, Y., Tomaru, Y., Takao, Y., Suzuki, H., Nagumo, T., Nagasaki, K., 2008. Isolation and characterization of a single-stranded RNA virus infecting the marine planktonic diatom Chaetoceros tenuissimus Meunier. Applied and Environmental Microbiology 74, 4022-4027.

Steward, G.F., Culley, A.I., Mueller, J.A., Wood-Charlson, E.M., Belcaid, M., Poisson, G., 2013. Are we missing half of the viruses in the ocean? Isme Journal 7, 672-679.

Suttle, C.A., Feng, C., 1992. Mechanisms and rates of decay of marine viruses in seawater. Applied and Environmental Microbiology 58, 3721-3729.

Taylor, R.L., AbrahamssonK., Godhe, A., Wa“ngberg, S, 2009. Seasonal Variability in Polyunsaturated Aldehyde Production Potential among Strains of Skeletonema Marinoi (Bacillariophyceae)1. Journal of Phycology 45, 46--53.

Teder, T., Lõhelaid, H., Boeglin, W.E., Calcutt, W.M., Brash, A.R., Samel, N., 2015. A Catalase-related Hemoprotein in Coral Is Specialized for Synthesis of Short-chain Aldehydes: Discovery of P450-type hydroperoxide lyase activity in a catalase. Journal of Biological Chemistry 290, 19823-19832.

Tomaru, Y., Takao, Y., Suzuki, H., Nagumo, T., Nagasaki, K., 2009. Isolation and Characterization of a SingleStranded RNA Virus Infecting the Bloom-Forming Diatom Chaetoceros socialis. Applied and Environmental Microbiology 75, 2375-2381.

Tomaru, Y., Toyoda, K., Kimura, K., Hata, N., Yoshida, M., Nagasaki, K., 2012. First evidence for the existence of pennate diatom viruses. Isme Journal 6, 1445-1448.

Tomaru, Y., Toyoda, K., Kimura, K., Takao, Y., Sakurada, K., Nakayama, N., Nagasaki, K., 2013. Isolation and characterization of a single-stranded RNA virus that infects the marine planktonic diatom Chaetoceros sp (SS08C03). Phycological Research 61, 27-36.

Toporkova, Y.Y., Gogolev, Y.V., Mukhtarova, L.S., Grechkin, A.N., 2008. Determinants governing the CYP74 catalysis: Conversion of allene oxide synthase into hydroperoxide lyase by site-directed mutagenesis. Febs Letters 582, 3423-3428.

Tsitsigiannis, D.I., Zarnowski, R., Keller, N.P., 2004. The lipid body protein, PpoA, coordinates sexual and asexual sporulation in Aspergillus nidulans. J Biol Chem 279, 11344-11353.

Underhill, E.W., Chisholm, M.D., Steck, W., 1980. (E)-5, (Z)-7-Dodecadienal, a sex pheromone component of the Western Tent Caterpillar, Malacosoma Californicum (Lepidoptera: Lasiocampidae). The Canadian Entomologist $112,629-631$.

Vardi, A., Bidle, K.D., Kwityn, C., Hirsh, D.J., Thompson, S.M., Callow, J.A., Falkowski, P., Bowler, C., 2008. A diatom gene regulating nitric-oxide signaling and susceptibility to diatom-derived aldehydes. Curr Biol 18, 895-899.

Vardi, A., Formiggini, F., Casotti, R., De Martino, A., Ribalet, F., Miralto, A., Bowler, C., 2006. A Stress Surveillance System Based on Calcium and Nitric Oxide in Marine Diatoms. PLoS Biol 4, e60.

Vidoudez, C., Casotti, R., Bastianini, M., Pohnert, G., 2011a. Quantification of dissolved and particulate polyunsaturated aldehydes in the Adriatic Sea. Marine drugs 9, 500-513.

Vidoudez, C., Nejstgaard, J.C., Jakobsen, H.H., Pohnert, G., 2011b. Dynamics of Dissolved and Particulate Polyunsaturated Aldehydes in Mesocosms Inoculated with Different Densities of the Diatom Skeletonema marinoi. Marine Drugs 9, 345-358. 
Wasternack, C., Feussner, I., 2008. Multifunctional Enzymes in Oxylipin Metabolism. Chembiochem 9, 2373-2375.

Weinberger, F., Lion, U., Delage, L., Kloareg, B., Potin, P., Beltran, J., Flores, V., Faugeron, S., Correa, J., Pohnert, G., 2011. Up-Regulation of Lipoxygenase, Phospholipase, and Oxylipin-Production in the Induced Chemical Defense of the Red Alga Gracilaria chilensis against Epiphytes. Journal of Chemical Ecology 37, 677-686.

Wendel, T., Jüttner, F., 1996. Lipoxygenase-mediated formation of hydrocarbons and unsaturated aldehydes in freshwater diatoms. Phytochemistry 41, 1445-1449.

Wennman, A., Jerneren, F., Magnuson, A., Oliw, E.H., 2015. Expression and characterization of manganese lipoxygenase of the rice blast fungus reveals prominent sequential lipoxygenation of alpha-linolenic acid. Archives of Biochemistry and Biophysics 583, 87-95. 
Figure 1. Diatom oxylipin biosynthesis pathways from the literature. A) Hexadecatrienoic acid pathway. Lipoxygenation of the $\mathrm{C} 16: 3$ free fatty acid $(\mathrm{HTrE} ; 1)$ at the 6 th and 9 th carbons yields 6 -hydroperoxy hexadecatrienoic acid (6-HpHTrE; 2) and 9-HpHTrE (3). HPL cleaves 6-HpHTrE to produce two hydroxyl acids, 6-hydroxy octenoic acid (4) and 8- hydroxy octenoic acid (5). Hydroperoxy lyase (HPL) can cleave 9-HpHTrE to produce the PUA octadienal (6) and 8-hydroxy octenoic acid (5). Allene oxide synthase (AOS) produces 11,9hydroxyepoxy hexadecadienoic acid (11,9-HepHDE;7) from 9-HpHTrE (3). Peroxidase activity reduces both 6- and 9- $\mathrm{HpHTrE}$ to the respective hydroxy acid, 6-HHTrE (8) and 9-HHTrE (9). 6-HpHTrE has also been observed to non-enzymatically covert to the keto acid 6-KHTrE (10).

B) Hexadecatetraenoic acid pathway. LOX enzymes add a hydroperoxyl group to the 6th and 9th carbon in the C16:4 free fatty acid (HTE; 11) forming 6-hydroperoxy hexatetraenoic acid (6-HpHTE; 12) and 9- HpHTE (13). HPL activity produces the PUA octatrienal (14) and 8-hydroxy octenoic acid (5) from 9-HpHTE (13). Similar to the C16:3 pathway, peroxidase activity reduces both 6- and 9- HpHTE to the respective hydroxy acids, 6-hydroxy hexadecatetranoic acid (6-HTE; 15) and 9-HHTE (16). 6-HpHTrE (12) has also been observed to non-enzymatically covert to 6-keto hexadecatetraenoic acid (6-KHTE; 17).

C) Arachidonic acid pathway (AA;18). Activity of LOX enzymes specific for the 11th and 12th carbons of the C20:4 free fatty acid yields (11R)-hydroperoxy eicosatetraenoic acid (HpETE;19) and 12-HpETE (20). HPL cleaves 11R-HpETE (19) to produce the PUA decadienal (21) and 10-hydroxy decadienoic acid (22), whereas, the peroxidase activity results in 11-HETE (23) production. Hydroperoxide halolyase (HPH) cleaves 12-HpETE (20) to produce 12-oxo dodecadienoic acid (24) and chlorinated alkenes (25).

D) Eicosapentaenoic acid pathway (EPA;26). LOX enzymes targeting the 5th, 9th, 11th, 12th, 14th, 15th, or 18th carbons of the C20:5 free fatty acid produce the respective hydroperoxy eicosapentaenoic acids (5-, 9-, 11R-, 12-, 14-, 15-, 18-HpETE; 27-33). HPL cleaves 9-HpETE (28) to produce 9-oxo nondienoic acid (34) and diatom hormones finavarrene (35) and hormosirene (36), 11R-HpEPE (29) to produce the PUA decatrienal (37) and 10hydroxy decadienoic acid (24), and 14-HpEPE (31) to produce the PUA heptadienal (38) and 13-hydroxy tridecatrienoic acid (39). AOS acts on 5-HpEPE (27) to produce 7,5 HepETE (40), 9-HpEPE (28) to produce 7,8HepETE (41), 14-HpEPE (31) to produce 13,14-HepETE (42) and 16,14 HepETE (43), 15-HpEPE (32) to produce 15,16 HepETE(44), and 18-HpEPE (33) to produce 16,17 HpETE (45). Peroxidase activity produces 5-, 9-, 11R-, 14-, 15-and 18-HEPE (46-51) from the respective HpEPE precursors. HPH activity cleaves 12-HpEPE (30) to produce 12-oxo dodecatrienoic acid (25) and a chlorinated alkene (52)

E) Docosahexenoic acid pathway (DHA; 53). LOX enzymes specific to the 17th and 20th carbon on the C22:6 free fatty acid produce 17- hydroperoxy docosahexenoic acid (17-HpDoHE; 54) and 20-HpDoHE (55). Peroxidation of 17- and 20-HpDoHE produces 17-hydroxy docosahexenoic acid (17-HDoHE; 56) and 20-HDoHE (57). Activity of AOS yields 15,16 hydroxy epoxy docosapentaenoic acid (15,16-HepDoPE; 58) from 17-HpDoHE (54) and 18,19HepDoPE (59) from 20-HpDoHE (55).

Adapted from Andreou et al. (2009) and Nanjappa et al. (2014). Note this figure does not encompass the entire diversity of molecules produced by diatoms. Additionally, this figure does not reflect the chirality or the cis-trans orientations of bonds. 


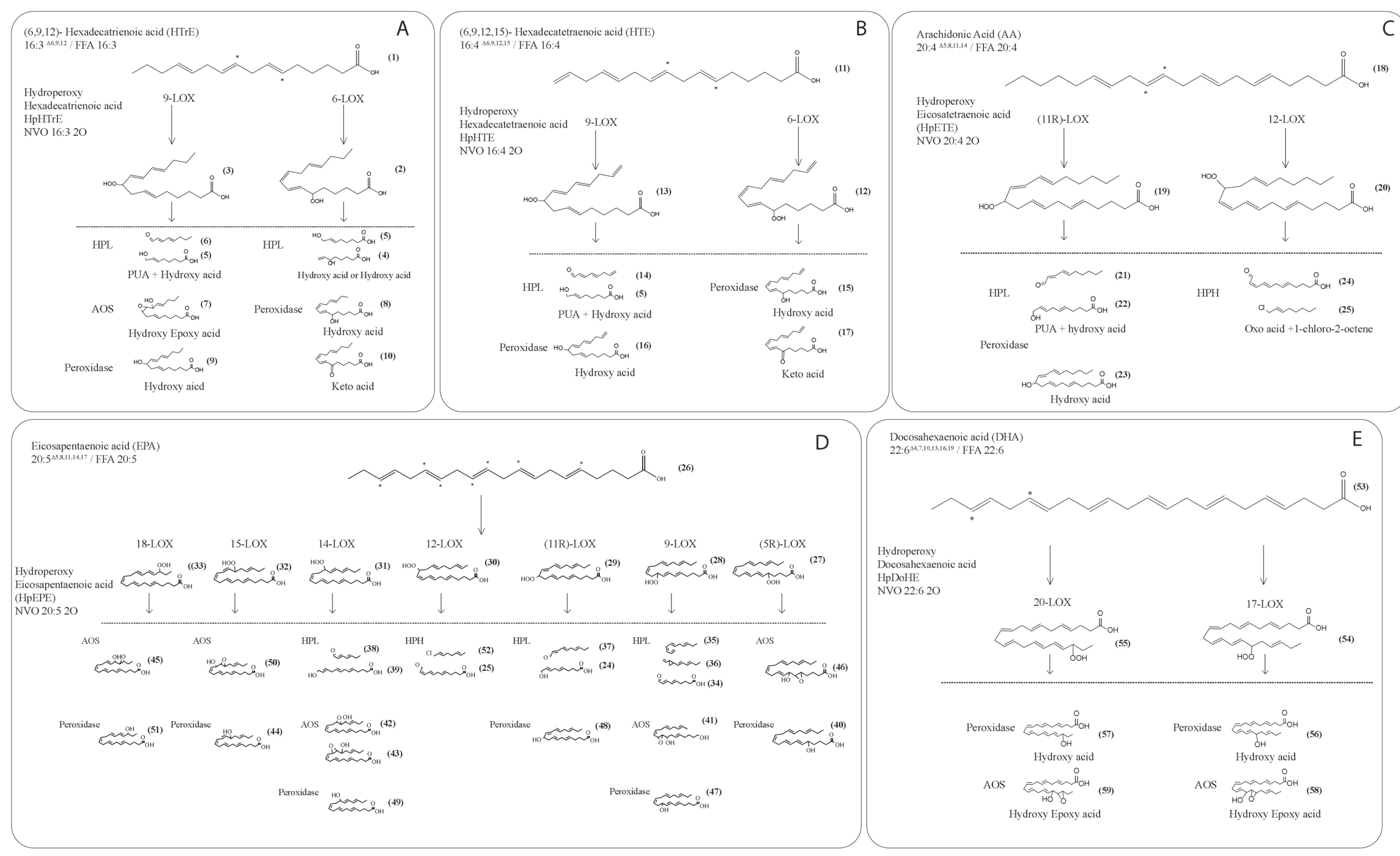



Figure 2. A) Nine stations were sampled in the Coastal California System. Stations 1, 2, 4, 8, 10, and 11 were located in the coastal upwelling region and Stations 6, 7, and 9 were off of the continental shelf. B) MODIS satellite image of chlorophyll concentration in the surface ocean during the DYEatom cruise with a sea surface altimetry overlay. Station 7 was situated in eddy on the other side of the California Current that had broken away from the upwelling region. 

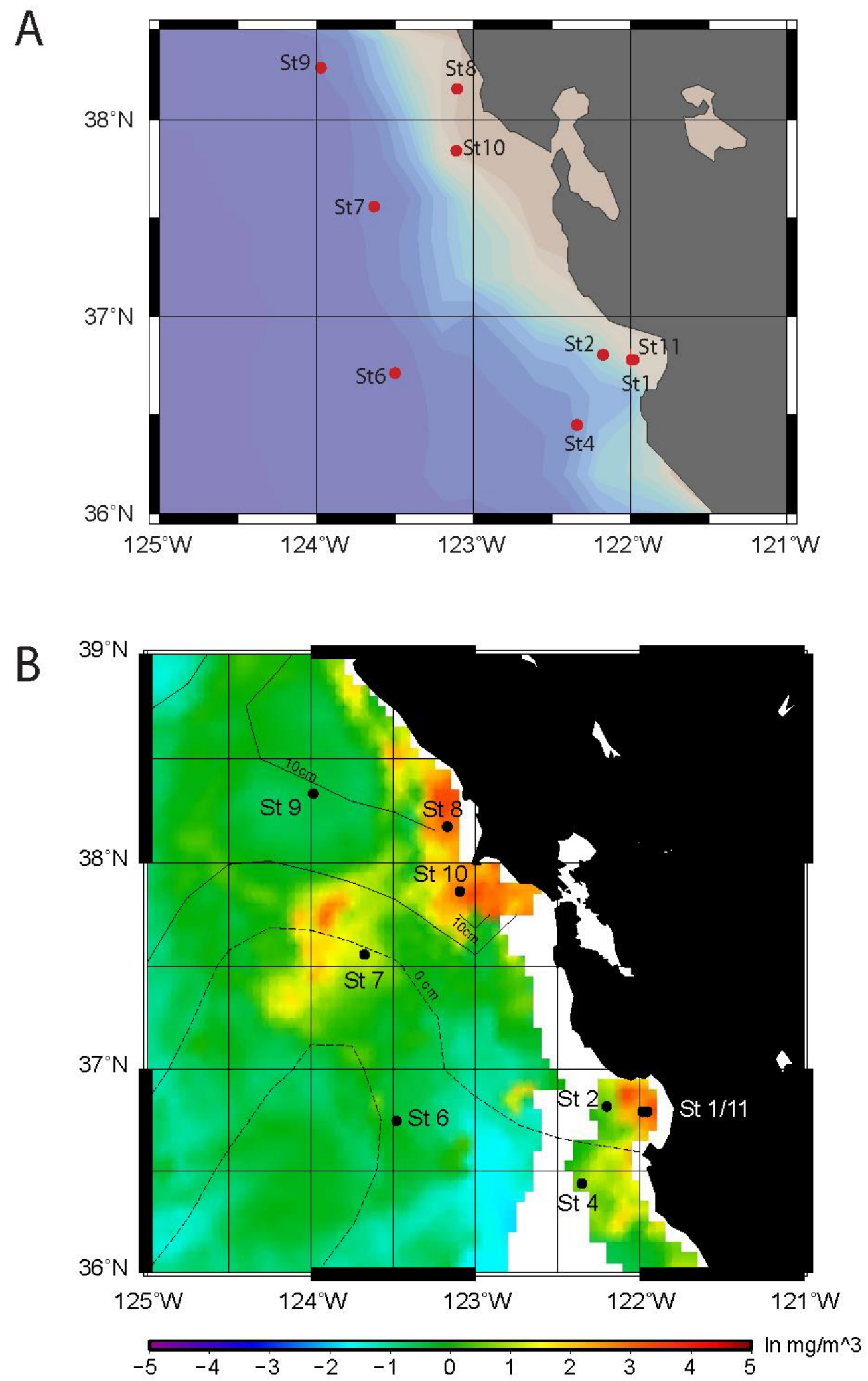

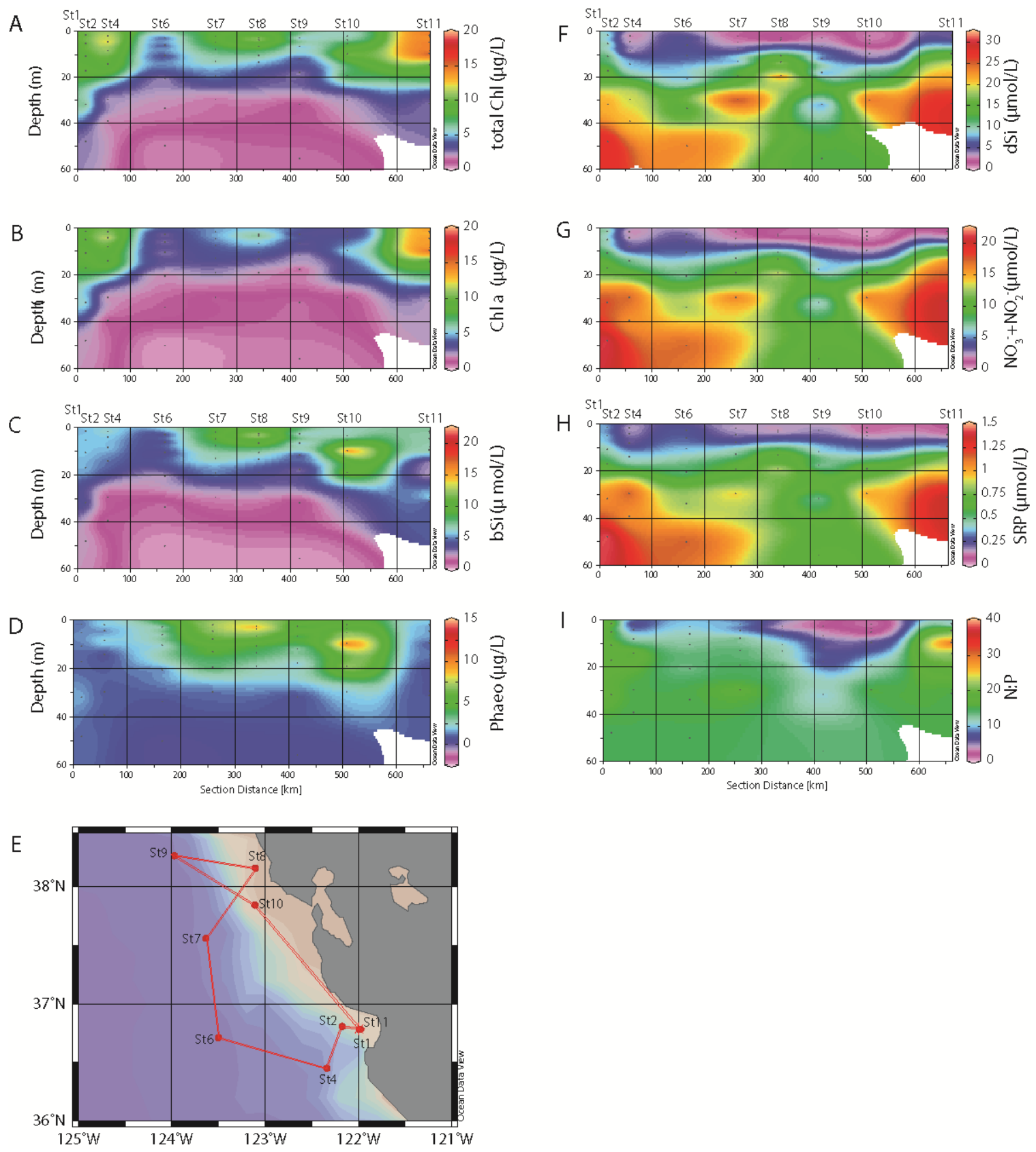

Figure 3. Sectional views of core variables measured throughout the upper water column along the DYEatom cruise track from St1 to St11. A) Total chlorophyll concentration $\mu \mathrm{g} \mathrm{L}^{-1}$ B) Chlorophyll a concentration $\mu \mathrm{g} \mathrm{L}^{-1} \mathrm{C}$ ) biogenic silica (bSi) concentration $\mu \mathrm{mol} \mathrm{L}{ }^{-1}$, an indicator of diatom biomass $\mathrm{D}$ ) phaeophytin concentration $\mu \mathrm{g} \mathrm{L}^{-1}$, an indicator of diatom demise E) Map of the cruise transect $\mathrm{F}$ ) dissolved silica $(\mathrm{dSi})$ concentration $\mu \mathrm{mol} \mathrm{L} \mathrm{L}^{-1} \mathrm{G}$ ) nitrogen concentration (nitrate and nitrite) $\mu \mathrm{mol} \mathrm{L}{ }^{-1} \mathrm{H}$ ) soluble reactive phosphorous (SRP) $\mu \mathrm{mol} \mathrm{L}^{-1}$ ) ratio of nitrogen to phosphorous (N:P). 

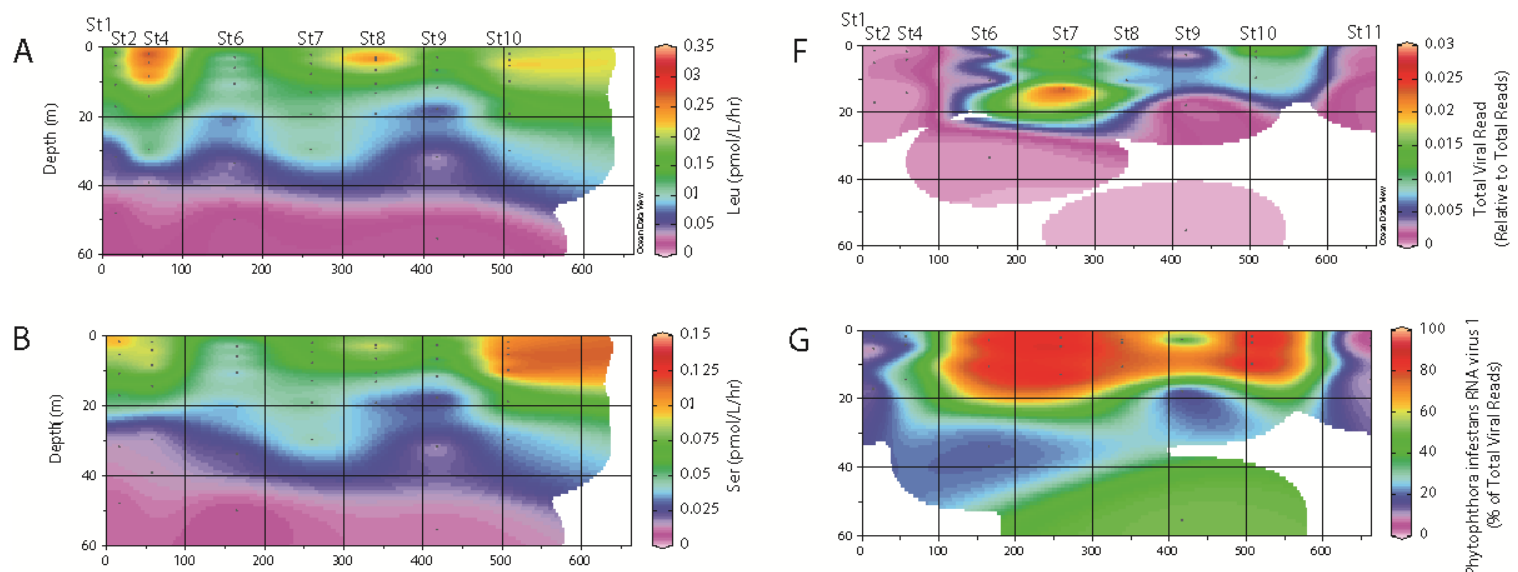

G
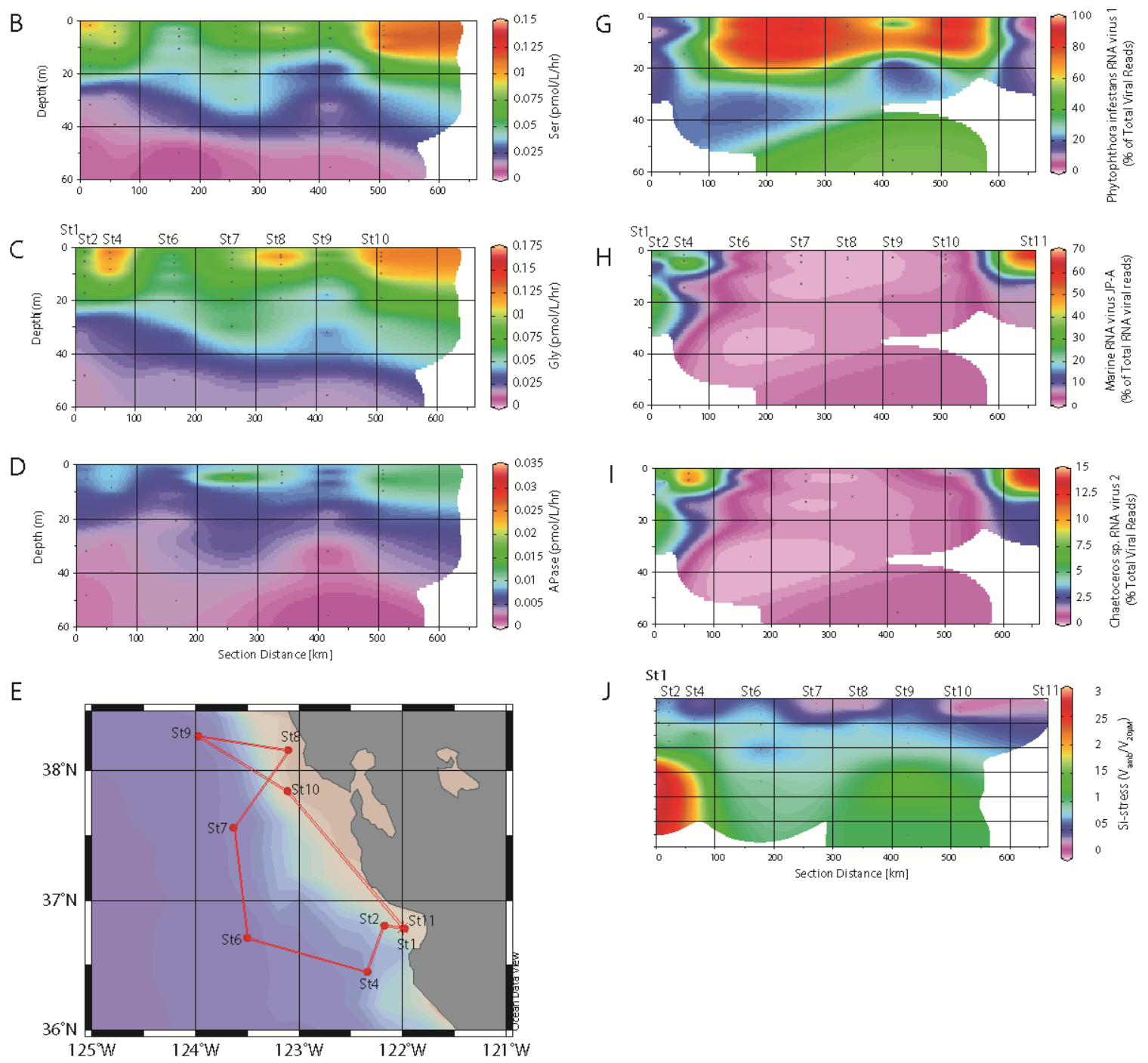

Figure 4. Sectional view of supplement variables measured at discrete depths in the water column along the DYEatom cruise track. A) enzymatic activity of leucine peptidase (Leu) pmol L-1 hr-1 B) enzymatic activity of serine peptidase (Ser) pmol L-1 hr-1 C) enzymatic activity of glycine peptidase (Gly) pmol L-1 hr-1 D) enzymatic activity of alkaline phosphatase (APase) pmol L-1 hr-1 E) Map of DYEatom cruise transect F) RNA Viral Reads as a proportion of total metatranscriptome reads G) Phytophora infestans RNA virus 1 as a percentage of total viral reads H) Marine RNA virus JP-A as a percentage of total viral reads I ) Chaetoceros sp. RNA virus 2 as a percentage of total viral reads J) silica stress reported as a ratio of the Silica uptake rate under ambient Si concentrations compared the rate of Si uptake upon at saturated concentrations 

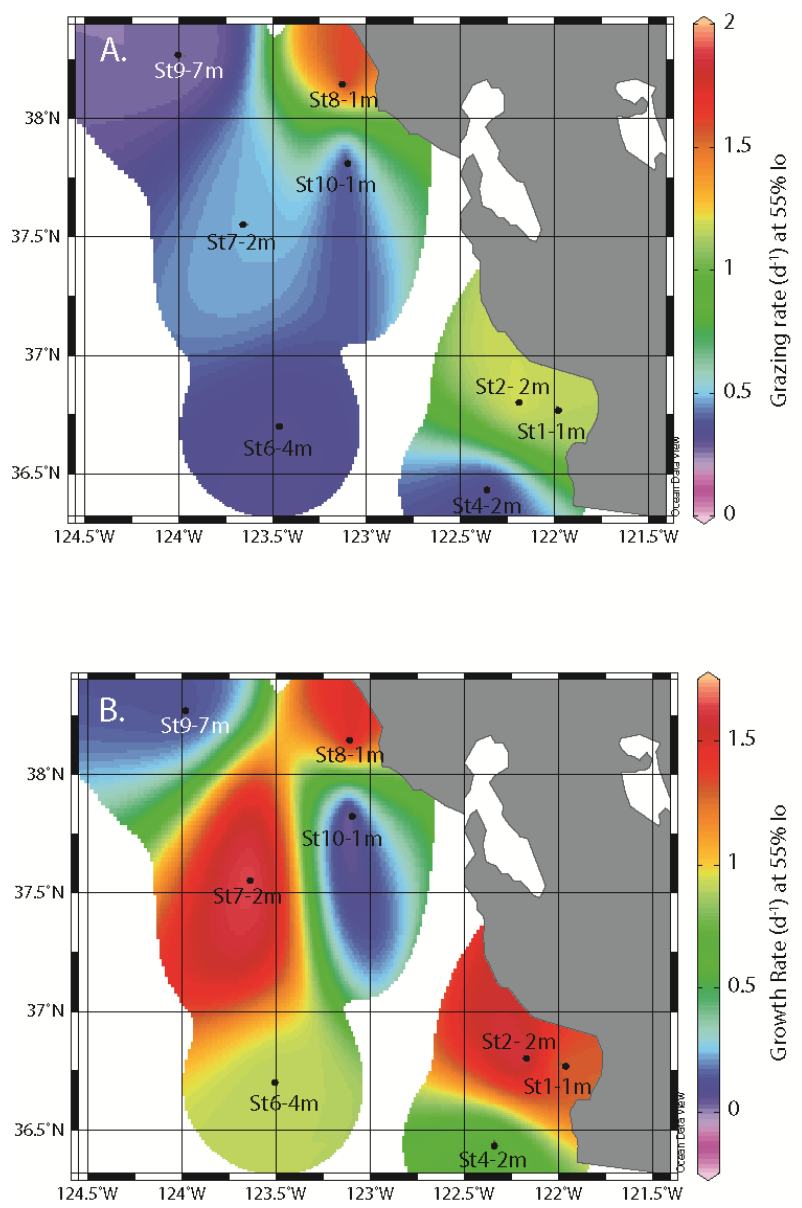

Figure 5. A) Microzooplankton grazing rates $\left(\mathrm{d}^{-1}\right)$ in the surface ocean along the cruise track B) Phytoplankton growth rates $\left(\mathrm{d}^{-1}\right)$ in the surface ocean along the DYEatom cruise track . 

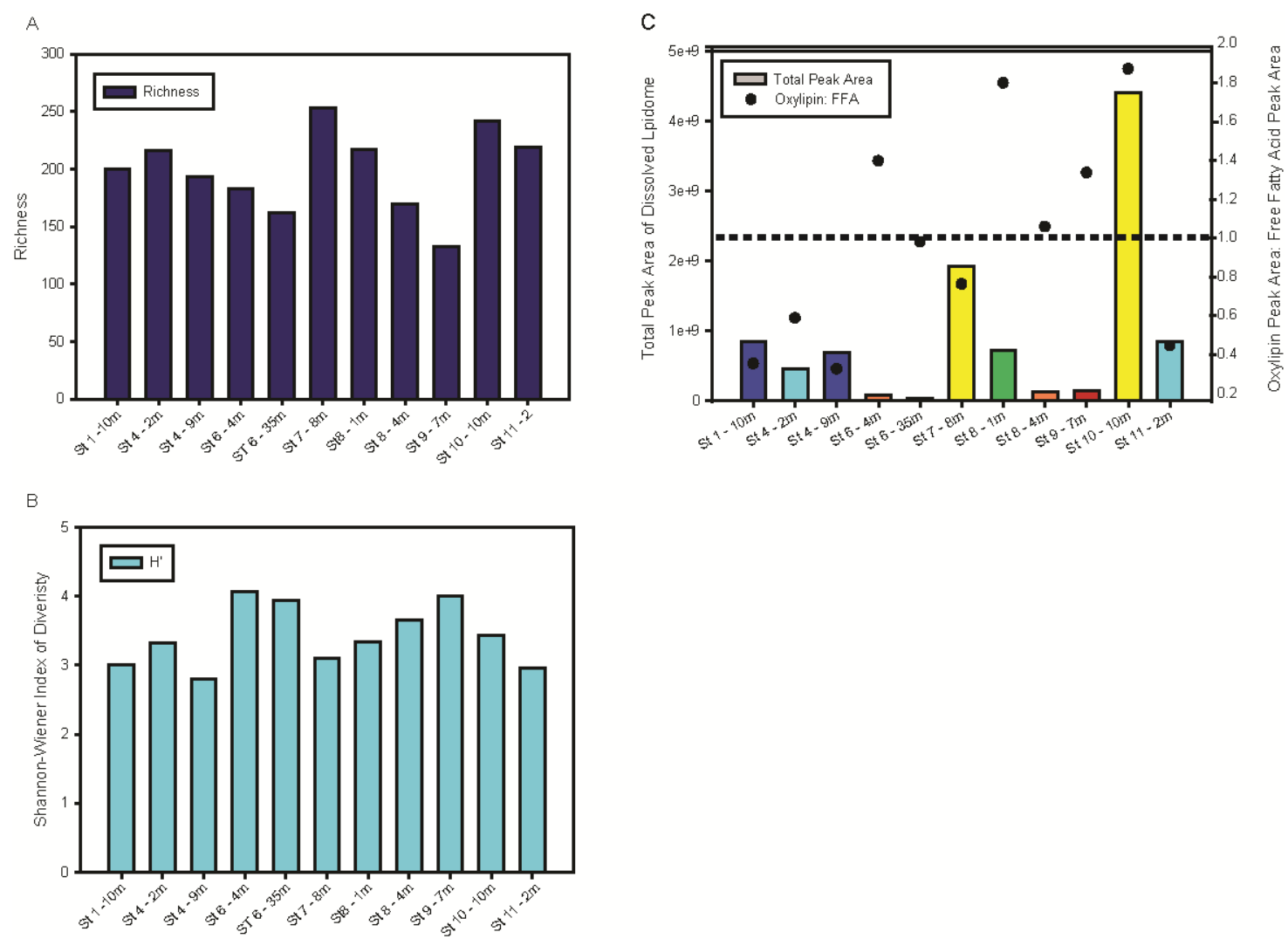

Figure 6. The richness, diversity, and abundance of molecules in the DYEatom cruise dissolved lipidome. A) Richness was determined by dunning the number of features in each sample. B) Diversity of the dissolved lipidome was determined using the Shannon-Wiener index (H). C) Total peak area of dissolved lipidome across the DYEatom cruise track accompanied by the ratio of oxylipin peak area to free fatty acid peak area. 


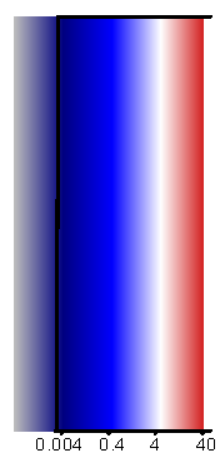

Percent of Total Peak Area

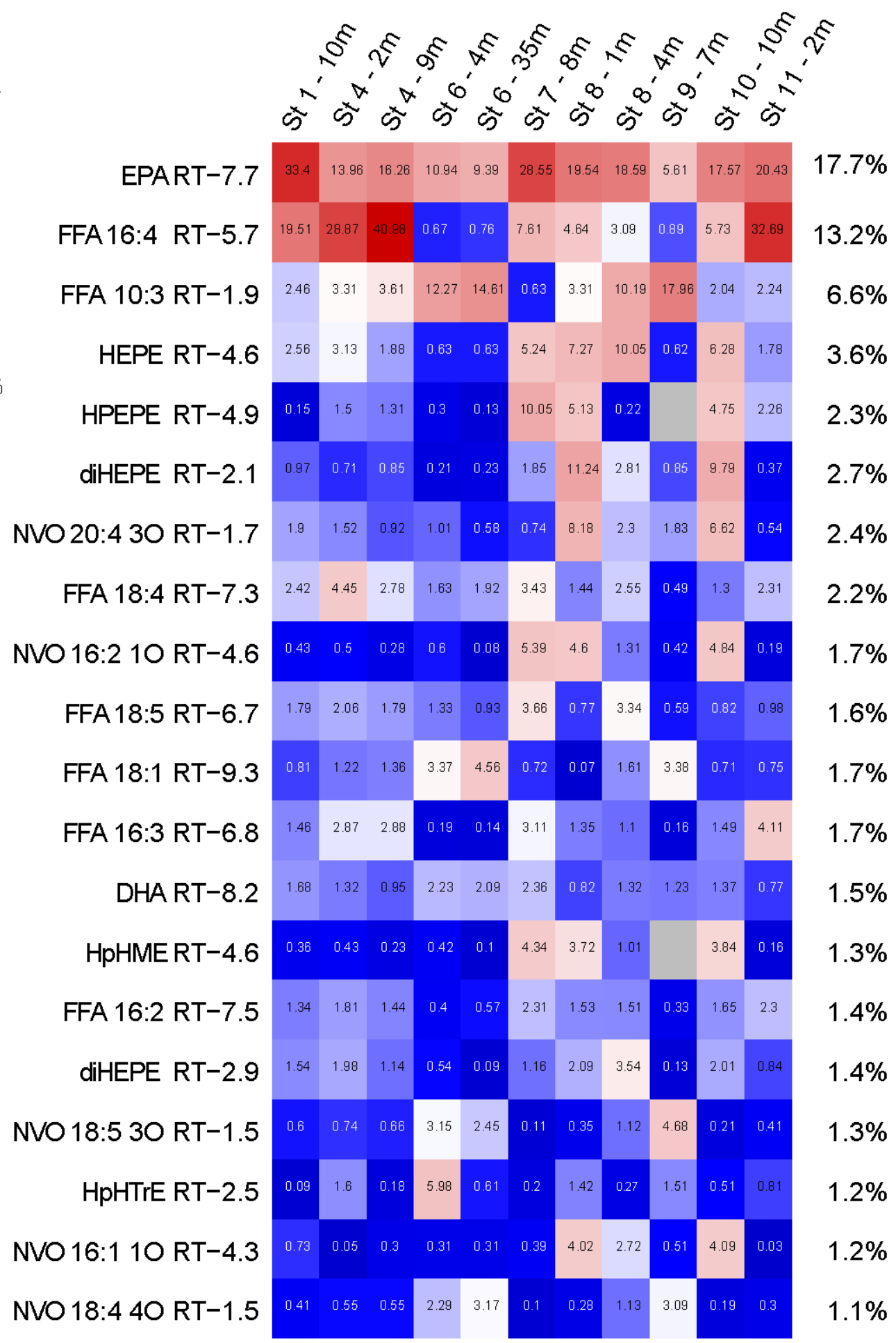

$.7 \%$

$6 \%$

$6 \%$

$3 \%$

$.7 \%$

$4 \%$

$.2 \%$

$1.7 \%$

$.6 \%$

$.7 \%$

$.7 \%$

$.5 \%$

$3 \%$

$4 \%$

$4 \%$

$3 \%$

$.2 \%$

$.2 \%$

$1.1 \%$

Figure 7. Composition of the features representing greater than $1 \%$ of the peak area in the annotated dissolved meta-lipidome. The heat map displays the percentage of the total peak area attributable to each molecular species across the samples. Functional group level annotations have been added for features in good agreement with the RT of the standards. 

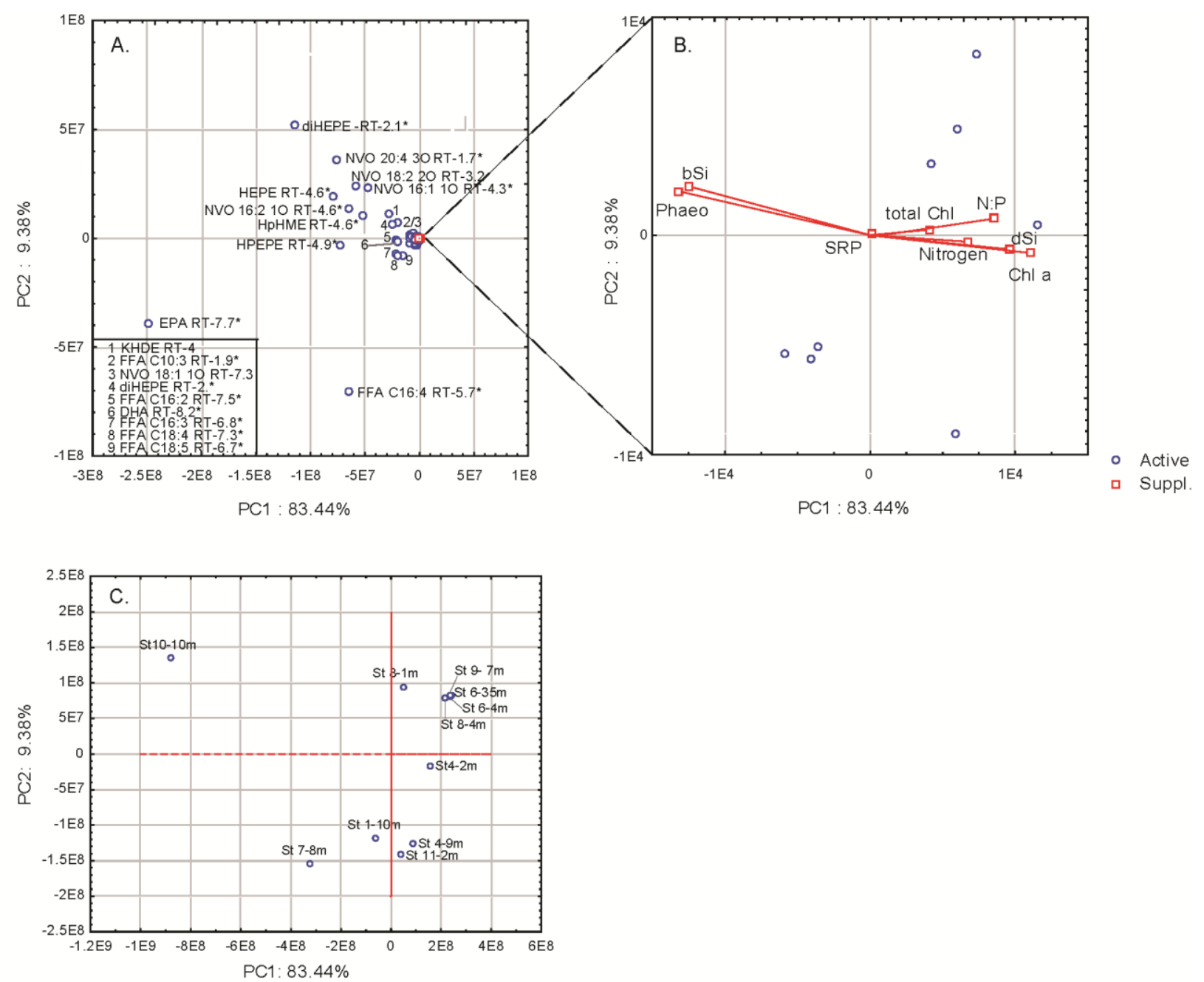

Figure 8. Covariance PCA of the DYEatom cruise dissolved lipidome. A) Loading of oxylipins (active variables) on principal component 1 (PC1; 83.44\% of the variation) and PC2 (9.38\%) B) Loading of core supplemental variables on PC1 and PC2 C) Loading of samples on PC1 and PC2. 
A.

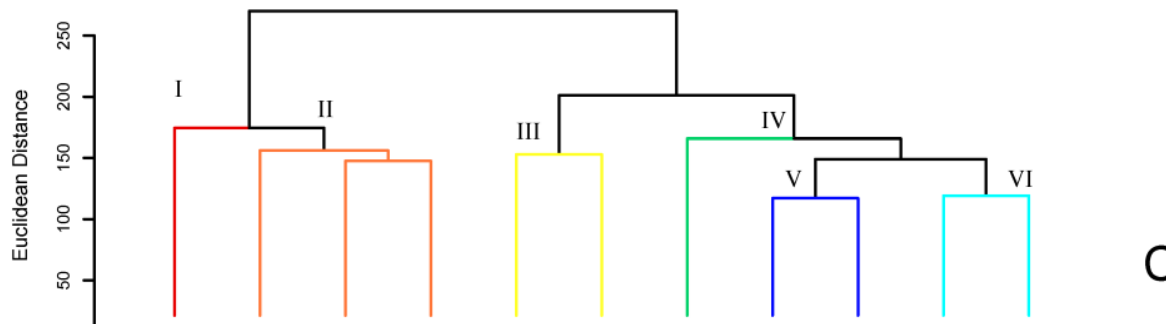

C.

B.
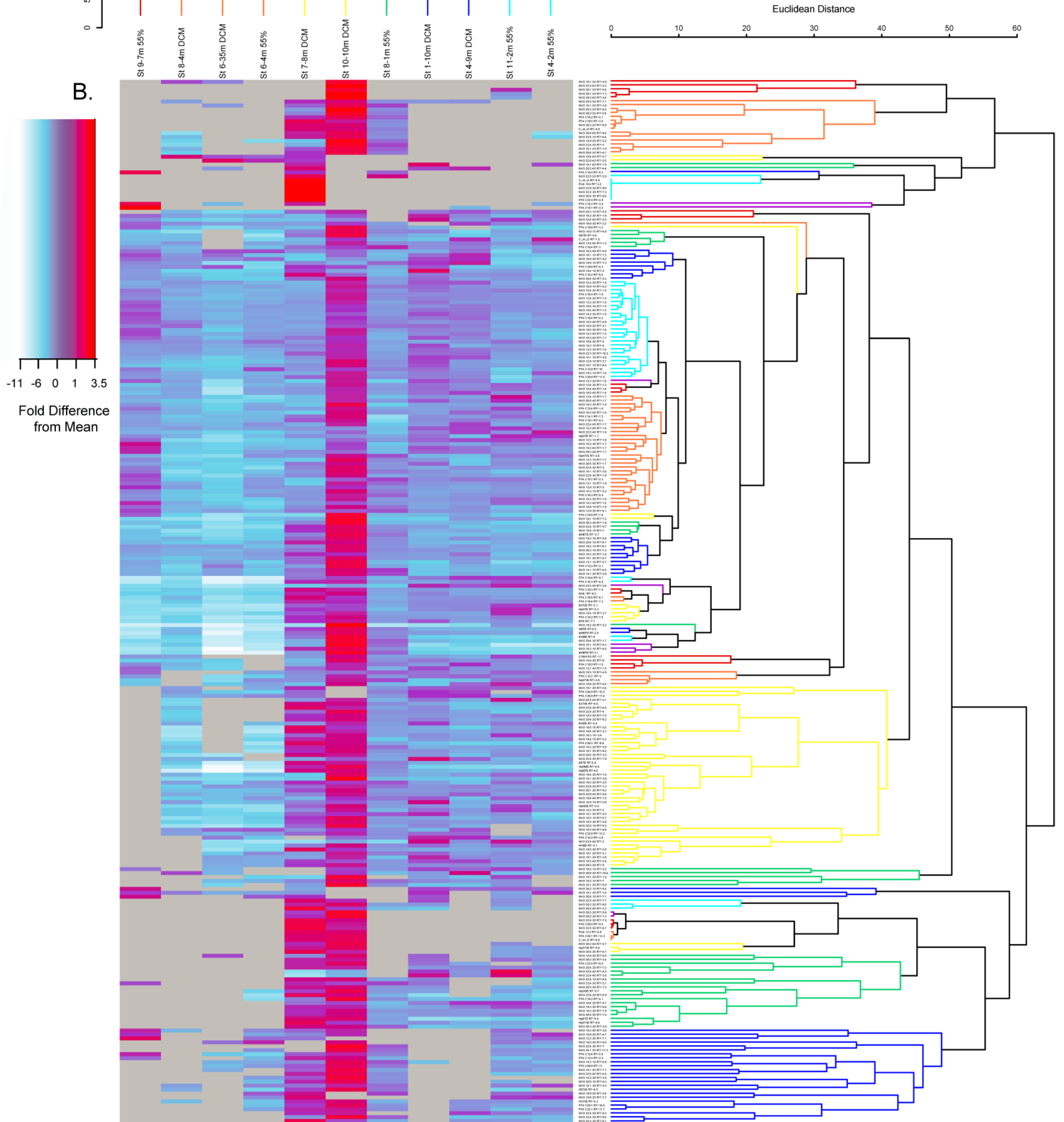

Figure 9. A) Similarity profile analysis of metalipidomic structure across the DYEatom cruise. The meta-lipidomes formed two branches with six significant clusters (Group I-VI) B) Heatmap displaying the fold difference from the mean for each feature in the meta-lipidome C) Similarity profile analysis of the fold difference in peak area of molecular species in the DYEatom meta-lipidome, forming two branches and forty significant clusters. 


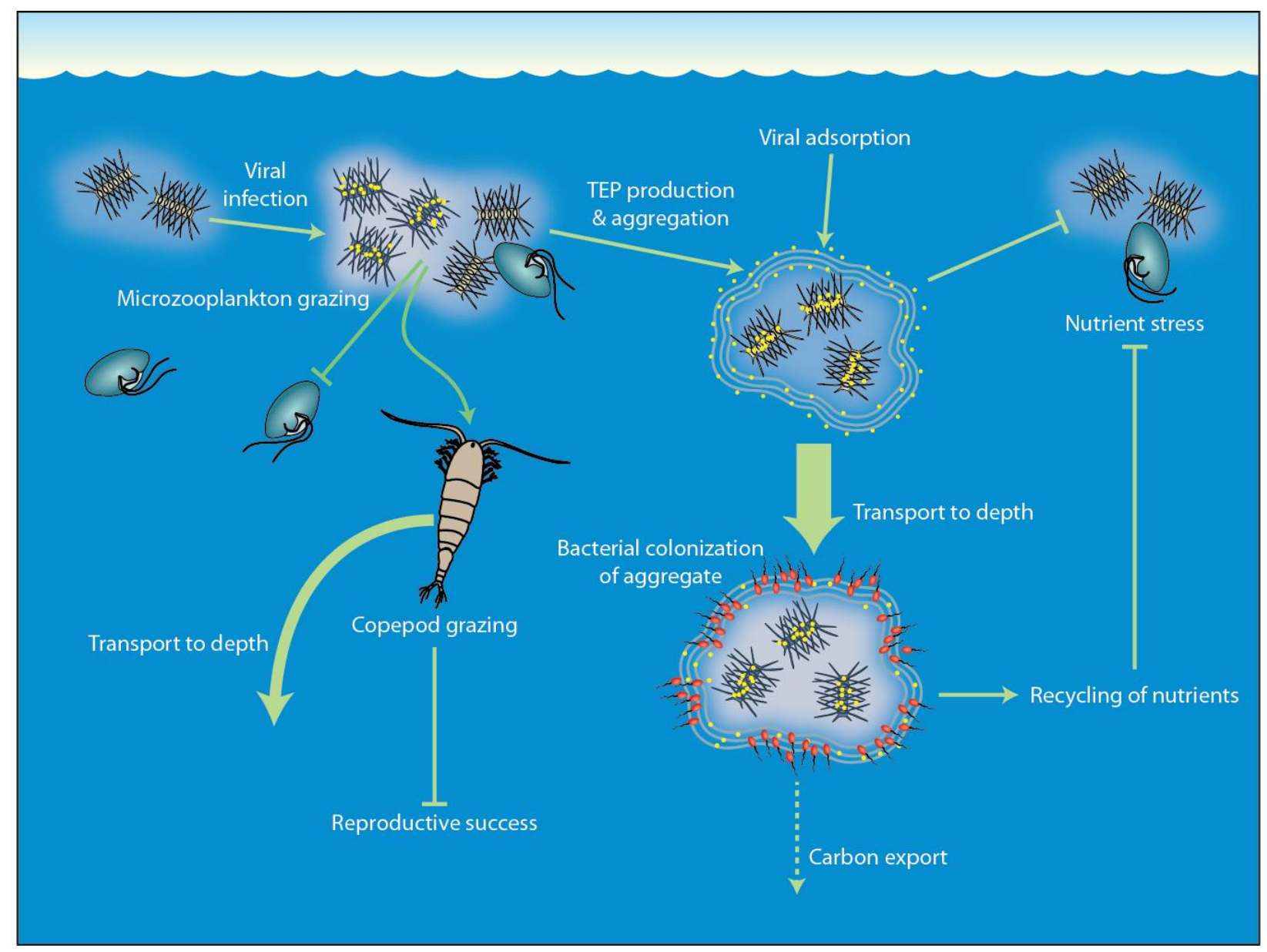

Figure 10. Conceptualization of virally induced oxylipin signaling and its hypothesized role in balancing diatom mortality. RNA viruses damage the cell, inducing oxylipin biosynthesis and release into the dissolved organic pool (represented by the white cloud). The suit of C20 and C16 non-volatile oxylipins that are produced deter microzooplankton grazing and suppress the copepod population. Oxylipins and senescence are also associated with transparent exopolymeric particle production (TEP) which causes phytoplankton cells to aggregate and settle out of the photic zone. Viruses readily adsorb to organic surfaces such as TEP thus they are transported out of the photic zone as well, reducing the encounter rate and staving off viral demise. The exported aggregates become hotspots for bacteria. Lysis of infected cells trapped in the aggregate, makes labile organic material more available to particle associated bacteria and increases the efficiency of the viral shunt. Oxylipins dissolved in the interstitial space of aggregates also stimulate bacterial recycling of nutrients. In a dynamic environment such as the California Coastal System, these nutrients could be injected back into the surface ocean. Nutrient replete environments decrease the intracellular capacity to produce oxylipins and thus nutrient recycling may serve as a negative feedback loop. 


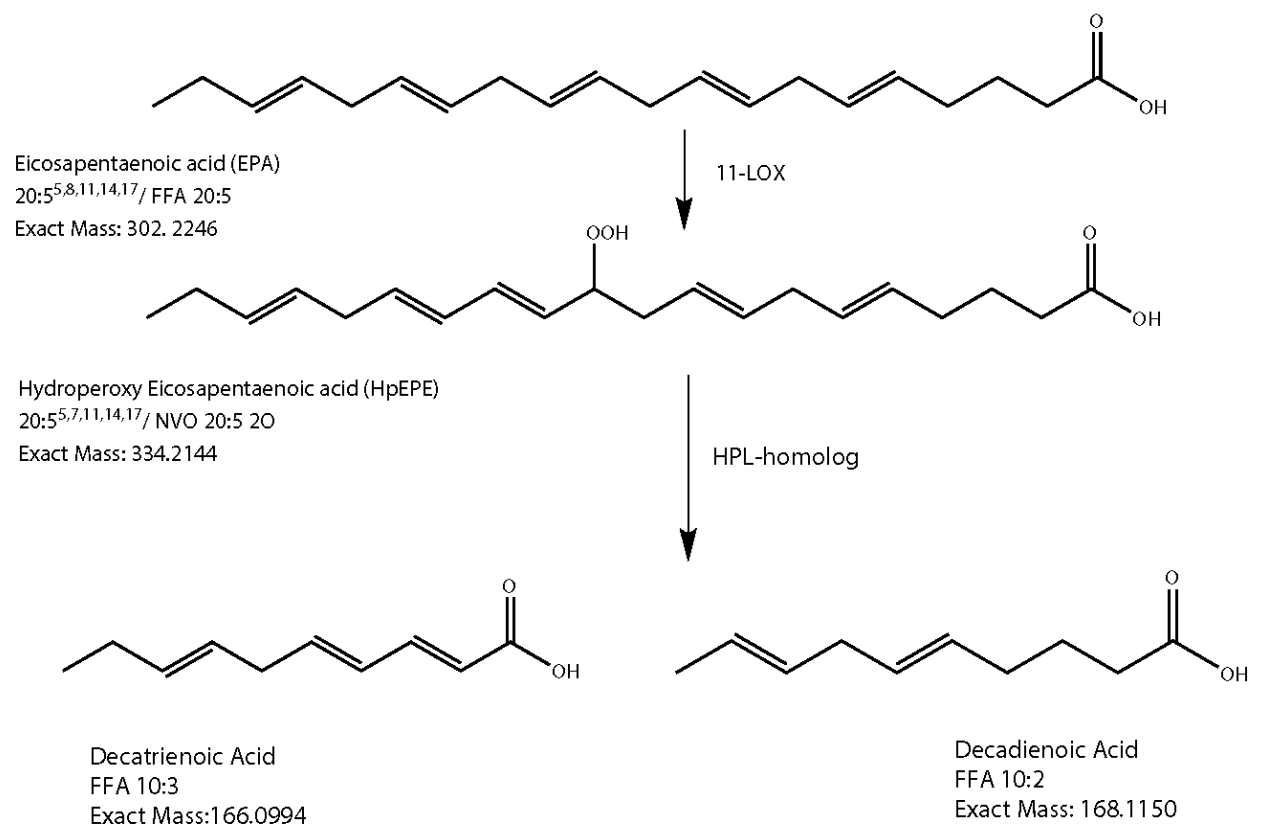

Figure 11. Proposed decatrienoic acid biosynthesis pathway. Hydroperoxy eicosapentaenoic acid is formed by lipoxygenation of EPA by the LOX enzyme. A hydroperoxylyase demonstrating functional plasticity cleaves HpEPE producing decatrienoic and decadienoic acids, known herbicides produced by terrestrial bacteria. 
Table 1. Supplemental data for dissolved lipidomic samples across the DYEatom cruise track

\begin{tabular}{|c|c|c|c|c|c|c|c|c|c|c|}
\hline Station & $\begin{array}{c}\text { Depth } \\
\text { (m) }\end{array}$ & Latitude N & Longitude W & Date & Io $\%$ & $\begin{array}{c}\text { Total } \\
\text { Chl } \\
(\mu \mathrm{g} / \mathrm{L})\end{array}$ & $\begin{array}{c}\text { Chl a } \\
(\mu \mathrm{g} / \mathrm{L})\end{array}$ & $\begin{array}{c}\mathrm{bSi} \\
(\mu \mathrm{mol} / \mathrm{L})\end{array}$ & $\begin{array}{l}\text { Phaeo } \\
(\mu \mathrm{g} / \mathrm{L})\end{array}$ & $\begin{array}{c}\mathrm{dSi} \\
(\mu \mathrm{mol} / \mathrm{L})\end{array}$ \\
\hline 1 & 10 & $36^{\circ} 46.864^{\prime}$ & $121^{\circ} 59.1257^{\prime}$ & June 27, 2013 & 1 & 19.157 & 19.19 & 8.379 & -0.064 & 17.313 \\
\hline 4 & 2 & $36^{\circ} 27.31^{\prime}$ & $122^{\circ} 20.32^{\prime}$ & June 29, 2013 & 55 & 12.213 & 10.873 & 6.69 & 2.6 & 1.598 \\
\hline 4 & 4 & $36^{\circ} 27.31^{\prime}$ & $122^{\circ} 20.32^{\prime}$ & June 29, 2013 & 6.6 & 12.235 & 10.77 & 5.903 & 2.842 & 2.571 \\
\hline 6 & 4 & $36^{\circ} 42.61^{\prime}$ & $123^{\circ} 29.62^{\prime}$ & June 30, 2013 & 55 & 0.94 & 0.754 & 1.242 & 0.362 & 6.489 \\
\hline 6 & 35 & $36^{\circ} 42.61^{\prime}$ & $123^{\circ} 29.62^{\prime}$ & June 30, 2013 & 1 & 1.634 & 1.298 & 0.271 & 0.652 & 13.823 \\
\hline 7 & 8 & $37^{\circ} 33.42^{\prime}$ & $123^{\circ} 37.96^{\prime}$ & July 1, 2013 & 6.6 & 7.574 & 3.453 & 8.425 & 7.993 & 1.885 \\
\hline 8 & 1 & $38^{\circ} 8.96^{\prime}$ & $123^{\circ} 5.95^{\prime}$ & July 2, 2013 & 55 & 13.589 & 7.17 & 16.879 & 12.452 & 0.469 \\
\hline 8 & 4 & $38^{\circ} 8.96^{\prime}$ & $123^{\circ} 5.95^{\prime}$ & July 2, 2013 & 22 & 15.403 & 9.991 & 17.146 & 10.498 & 0.8 \\
\hline 9 & 7 & $38^{\circ} 15.89^{\prime}$ & $123^{\circ} 58.12^{\prime}$ & July 3, 2013 & 55 & 0.291 & 0.176 & 0.208 & 0.224 & 3.536 \\
\hline 10 & 10 & $37^{\circ} 50.24^{\prime}$ & $123^{\circ} 6.6^{\prime}$ & July 4, 2013 & 1 & 13.487 & 6.485 & 21.701 & 13.583 & 3.243 \\
\hline 11 & 2 & $36^{\circ} 46.67^{\prime}$ & $121^{\circ} 58.83^{\prime}$ & July 5, 2013 & 55 & 12.012 & 11.835 & 5.563 & 0.345 & 2.847 \\
\hline
\end{tabular}


Table 1. (continued) Supplemental data for metalipidomic samples across the DYEatom cruise track

\begin{tabular}{|c|c|c|c|c|c|c|c|c|c|}
\hline Station & $\begin{array}{c}\text { Depth } \\
\text { (m) }\end{array}$ & $\begin{array}{c}\text { SRP } \\
(\mu \mathrm{mol} / \mathrm{L})\end{array}$ & $\begin{array}{c}\mathrm{NO} 3+\mathrm{NO} 2 \\
(\mu \mathrm{mol} / \mathrm{L})\end{array}$ & $\mathrm{N}: \mathrm{P}$ & $\begin{array}{c}\text { Si-stress } \\
(\% \\
\text { Vamb }) \\
\end{array}$ & $\begin{array}{c}\text { Leu } \\
\text { (pmol/L/hr) }\end{array}$ & $\begin{array}{c}\text { Ser } \\
\text { (pmol/L/hr) }\end{array}$ & $\begin{array}{c}\text { Gly } \\
\text { (pmol/L/hr) }\end{array}$ & $\begin{array}{c}\text { APase } \\
\text { (pmol/L/hr) }\end{array}$ \\
\hline 1 & 10 & 0.277 & 11.043 & 39.87 & $99 \%$ & & & & \\
\hline 4 & 2 & 0.094 & 0.234 & 2.49 & $17 \%$ & $3.21 \mathrm{E}-01$ & $8.44 \mathrm{E}-02$ & $1.51 \mathrm{E}-01$ & $9.70 \mathrm{E}-03$ \\
\hline 4 & 4 & 0.114 & 1.231 & 10.80 & $61 \%$ & 3.03E-01 & $9.87 \mathrm{E}-02$ & $1.42 \mathrm{E}-01$ & $9.92 \mathrm{E}-03$ \\
\hline 6 & 4 & 0.364 & 3.857 & 10.60 & $74 \%$ & 4.59E-02 & $2.57 \mathrm{E}-02$ & 3.64E-02 & $3.10 \mathrm{E}-03$ \\
\hline 6 & 35 & 0.784 & 11.667 & 14.88 & $96 \%$ & $2.50 \mathrm{E}-02$ & $1.67 \mathrm{E}-02$ & $2.48 \mathrm{E}-02$ & $3.70 \mathrm{E}-03$ \\
\hline 7 & 8 & 0.097 & 1.759 & 18.13 & $29 \%$ & $1.32 \mathrm{E}-01$ & 7.22E-02 & $8.85 \mathrm{E}-02$ & $5.70 \mathrm{E}-03$ \\
\hline 8 & 1 & 0.09 & 0.043 & 0.48 & $10 \%$ & $3.44 \mathrm{E}-01$ & $1.24 \mathrm{E}-01$ & $1.52 \mathrm{E}-01$ & $1.28 \mathrm{E}-02$ \\
\hline 8 & 4 & 0.104 & 0.098 & 0.94 & $13 \%$ & $3.21 \mathrm{E}-01$ & $1.03 \mathrm{E}-01$ & $1.70 \mathrm{E}-01$ & $8.10 \mathrm{E}-03$ \\
\hline 9 & 7 & 0.219 & 0.13 & 0.59 & $70 \%$ & $2.24 \mathrm{E}-02$ & $1.26 \mathrm{E}-02$ & $2.07 \mathrm{E}-02$ & $2.30 \mathrm{E}-03$ \\
\hline 10 & 10 & 0.295 & 1.955 & 6.63 & $78 \%$ & $2.26 \mathrm{E}-01$ & $1.44 \mathrm{E}-01$ & $1.32 \mathrm{E}-01$ & $1.11 \mathrm{E}-02$ \\
\hline 11 & 2 & 0.071 & 0.593 & 8.35 & & & & & \\
\hline
\end{tabular}


Table 1. (continued) Supplemental data for metalipidomic samples across the DYEatom cruise track

\begin{tabular}{|c|c|c|c|c|c|c|c|c|}
\hline Station & $\begin{array}{c}\text { Depth } \\
\text { (m) }\end{array}$ & $\begin{array}{c}\text { Grazing } \\
\text { Rate } \\
(\mathrm{d}-1)\end{array}$ & $\begin{array}{c}\text { Growth Rate } \\
\text { (d-1) }\end{array}$ & $\begin{array}{l}\text { Phytophthora } \\
\text { infestans } \\
\text { RNA virus } 1 \\
\text { (\% of Total } \\
\text { RNA Viral } \\
\text { Reads) } \\
\end{array}$ & $\begin{array}{c}\text { Marine } \\
\text { RNA virus } \\
\text { JP-A (\% of } \\
\text { Total RNA } \\
\text { viral reads) } \\
\end{array}$ & $\begin{array}{c}\text { Chaetoceros } \\
\text { sp. RNA } \\
\text { virus } 2(\% \\
\text { Total RNA } \\
\text { Viral } \\
\text { Reads) } \\
\end{array}$ & $\begin{array}{l}\text { Total Viral } \\
\text { Reads } \\
\text { (relative to } \\
\text { total reads) }\end{array}$ & $\begin{array}{l}\text { Dissolved } \\
\text { Meta- } \\
\text { lipidome } \\
\text { (Total Peak } \\
\text { Area) } \\
\end{array}$ \\
\hline 1 & 10 & & & & & & & $8.43 E+08$ \\
\hline 4 & 2 & 0.31 & 0.51 & 24.63 & 10.97 & 11.73 & 0.000342787 & $4.64 \mathrm{E}+08$ \\
\hline 4 & 4 & & & & & & & $6.97 \mathrm{E}+08$ \\
\hline 6 & 4 & 0.33 & 0.89 & 88.15 & 0.25 & 0.03 & 0.000945634 & $7.88 \mathrm{E}+07$ \\
\hline 6 & 35 & & & 20.02 & 0.43 & 0.00 & 0.000276928 & $4.28 \mathrm{E}+07$ \\
\hline 7 & 8 & & & & & & & $1.92 \mathrm{E}+09$ \\
\hline 8 & 1 & 1.80 & 1.73 & 80.10 & 0.00 & 0.00 & 0.003182939 & $7.23 \mathrm{E}+08$ \\
\hline 8 & 4 & & & 81.25 & 0.13 & 0.00 & 0.004309564 & $1.33 \mathrm{E}+08$ \\
\hline 9 & 7 & 0.25 & 0.12 & & & & & $1.48 \mathrm{E}+08$ \\
\hline 10 & 10 & & & 89.80 & 0.73 & 0.24 & 0.008934868 & $4.41 \mathrm{E}+09$ \\
\hline 11 & 2 & & & 1.16 & 66.06 & 14.81 & 0.002408916 & $8.48 \mathrm{E}+08$ \\
\hline
\end{tabular}


Table 2. RNA viral metatranscriptomic hits to proteins predicted from known RNA viral genomes

\begin{tabular}{|c|c|c|c|c|}
\hline Species & Accession \# & Annotation & $\begin{array}{l}\text { Percent } \\
\text { Identity }\end{array}$ & $\begin{array}{c}\text { GOS } \\
\text { core } \\
\text { cluster }\end{array}$ \\
\hline Acanthamoeba polyphaga mimivirus & $\begin{array}{l}\text { gi55819520- } \\
\text { NC_006450 }\end{array}$ & hypothetical protein & 25.13 & \\
\hline Acanthamoeba polyphaga mimivirus & $\begin{array}{l}\text { gi55819641- } \\
\text { NC_006450 }\end{array}$ & hypothetical protein & 25.23 & \\
\hline $\begin{array}{l}\text { Antheraea pernyi nucleopolyhedrovirus } \\
\text { (AnpeNPV) }\end{array}$ & $\begin{array}{l}\text { gi96979849- } \\
\text { NC_008035 }\end{array}$ & Desmoplakin & 25.9 & $\mathrm{x}$ \\
\hline Aphid lethal paralysis virus & $\begin{array}{l}\text { gi24943168- } \\
\text { NC_004365 }\end{array}$ & nonstructural polyprotein & 31.95 & $\mathrm{x}$ \\
\hline Apple latent spherical virus & $\begin{array}{l}\text { gi20451025- } \\
\text { NC_003787 }\end{array}$ & $235 \mathrm{~K}$ polyprotein & 28.65 & $\mathrm{x}$ \\
\hline Apple latent spherical virus & $\begin{array}{l}\text { gi20451025- } \\
\text { NC_003787 }\end{array}$ & $235 \mathrm{~K}$ polyprotein & 32.31 & $\mathrm{x}$ \\
\hline Ateline herpesvirus 3 & $\begin{array}{l}\text { gi9631239- } \\
\text { NC_001987 }\end{array}$ & hypothetical protein & 31.21 & $\mathrm{x}$ \\
\hline Avian encephalomyelitis virus & $\begin{array}{l}\text { gi21389215- } \\
\text { NC_003990 }\end{array}$ & aev polyprotein & 27.72 & $\mathrm{x}$ \\
\hline Avian myelocytomatosis virus & $\begin{array}{l}\text { gi9629901- } \\
\text { NC_001866 }\end{array}$ & $\mathrm{p} 110$ & 37.17 & $\mathrm{x}$ \\
\hline Basella rugose mosaic virus & $\begin{array}{l}\text { gi156447516- } \\
\text { NC_009741 }\end{array}$ & polyprotein & 43.86 & $\mathrm{x}$ \\
\hline Bathycoccus sp. RCC1105 virus BpV1 & $\begin{array}{l}\text { BpV1_051- } \\
\text { NC_014765 }\end{array}$ & hypothetical protein & 37.04 & $\mathrm{x}$ \\
\hline Black raspberry necrosis virus & $\begin{array}{l}\text { gi109240523- } \\
\text { NC_008182 }\end{array}$ & polyprotein & 33.99 & $\mathrm{x}$ \\
\hline Black raspberry necrosis virus & $\begin{array}{l}\text { gi109240523- } \\
\text { NC_008182 }\end{array}$ & polyprotein & 33.99 & $\mathrm{x}$ \\
\hline Blackberry virus Y & $\begin{array}{l}\text { gi117413399- } \\
\text { NC_008558 }\end{array}$ & polyprotein & 29.69 & $\mathrm{x}$ \\
\hline Cafeteria roenbergensis virus BV-PW1 & $\begin{array}{l}\text { crov302- } \\
\text { NC_014637 }\end{array}$ & $\begin{array}{l}\text { putative AAA+ family } \\
\text { ATPase }\end{array}$ & 36.05 & $\mathrm{x}$ \\
\hline Calicivirus pig/AB90/CAN & $\begin{array}{l}\text { gi228551554- } \\
\text { FJ355928 }\end{array}$ & polyprotein & 27.27 & $\mathrm{x}$ \\
\hline
\end{tabular}


Table 2. (cont) RNA viral metatranscriptomic hits to proteins predicted from known RNA viral genomes

\begin{tabular}{|c|c|c|c|c|}
\hline Species & Accession \# & Annotation & $\begin{array}{l}\text { Percent } \\
\text { Identity }\end{array}$ & $\begin{array}{c}\text { GOS } \\
\text { core } \\
\text { cluster }\end{array}$ \\
\hline Chaetoceros sp. RNA virus 2 & $\begin{array}{l}\text { gi336391069- } \\
\text { AB639040 }\end{array}$ & $\begin{array}{l}\text { putative replication } \\
\text { related protein }\end{array}$ & 34.35 & \\
\hline Chaetoceros sp. RNA virus 2 & $\begin{array}{l}\text { gi336391069- } \\
\text { AB639040 }\end{array}$ & $\begin{array}{l}\text { putative replication } \\
\text { related protein }\end{array}$ & 34.35 & \\
\hline Chaetoceros sp. RNA virus 2 & $\begin{array}{l}\text { gi336391069- } \\
\text { AB639040 }\end{array}$ & $\begin{array}{l}\text { putative replication } \\
\text { related protein }\end{array}$ & 26.7 & \\
\hline Chaetoceros sp. RNA virus 2 & $\begin{array}{l}\text { gi336391069- } \\
\text { AB639040 }\end{array}$ & $\begin{array}{l}\text { putative replication } \\
\text { related protein }\end{array}$ & 47.85 & \\
\hline Chaetoceros sp. RNA virus 2 & $\begin{array}{l}\text { gi336391070- } \\
\text { AB639040 }\end{array}$ & putative structural protein & 41.6 & \\
\hline Chaetoceros sp. RNA virus 2 & $\begin{array}{l}\text { gi336391070- } \\
\text { AB639040 }\end{array}$ & putative structural protein & 35.96 & \\
\hline Chaetoceros sp. RNA virus 2 & $\begin{array}{l}\text { gi336391069- } \\
\text { AB639040 }\end{array}$ & $\begin{array}{l}\text { putative replication } \\
\text { related protein }\end{array}$ & 30.36 & \\
\hline Cotesia congregata bracovirus & $\begin{array}{l}\text { gi57753381- } \\
\text { NC_006649 }\end{array}$ & hypothetical protein & 34.02 & $\mathrm{x}$ \\
\hline Cricket paralysis virus & $\begin{array}{l}\text { gi21321710- } \\
\text { NC_003924 }\end{array}$ & structural polyprotein & 30.77 & $\mathrm{x}$ \\
\hline Cyanophage PSS2 & $\begin{array}{l}\text { gi254729546- } \\
\text { NC_013021 }\end{array}$ & hypothetical protein & 38.96 & $\mathrm{x}$ \\
\hline Cyanophage PSS2 & $\begin{array}{l}\text { gi254729546- } \\
\text { NC_013021 }\end{array}$ & hypothetical protein & 40.68 & $\mathrm{x}$ \\
\hline Cyanophage PSS2 & $\begin{array}{l}\text { gi254729546- } \\
\text { NC_013021 }\end{array}$ & hypothetical protein & 32.47 & $\mathrm{x}$ \\
\hline Cyanophage PSS2 & $\begin{array}{l}\text { gi254729546- } \\
\text { NC_013021 }\end{array}$ & hypothetical protein & 24.79 & $\mathrm{x}$ \\
\hline Drosophila $\mathrm{C}$ virus & $\begin{array}{l}\text { gi9629652- } \\
\text { NC_001834 }\end{array}$ & capsid polyprotein & 30 & $\mathrm{x}$ \\
\hline Drosophila $\mathrm{C}$ virus & $\begin{array}{l}\text { gi9629651- } \\
\text { NC_001834 }\end{array}$ & replicase polyprotein & 35.26 & $\mathrm{x}$ \\
\hline Drosophila $\mathrm{C}$ virus & $\begin{array}{l}\text { gi9629651- } \\
\text { NC_001834 }\end{array}$ & replicase polyprotein & 24.92 & $\mathrm{x}$ \\
\hline
\end{tabular}


Table 2. (cont) RNA viral metatranscriptomic hits to proteins predicted from known RNA viral genomes

\begin{tabular}{|c|c|c|c|c|}
\hline Species & Accession \# & Annotation & $\begin{array}{l}\text { Percent } \\
\text { Identity }\end{array}$ & $\begin{array}{l}\text { GOS } \\
\text { core } \\
\text { cluster }\end{array}$ \\
\hline Drosophila $\mathrm{C}$ virus & $\begin{array}{l}\text { gi9629652- } \\
\text { NC_001834 }\end{array}$ & capsid polyprotein & 35.9 & $\mathrm{x}$ \\
\hline Duck hepatitis A virus & $\begin{array}{l}\text { gi110611895- } \\
\text { NC_008250 }\end{array}$ & polyprotein & 25.19 & $\mathrm{x}$ \\
\hline Emiliania huxleyi virus 86 & $\begin{array}{l}\text { gi73852696- } \\
\text { NC_007346 }\end{array}$ & hypothetical protein & 38.24 & \\
\hline $\begin{array}{l}\text { Helicoverpa armigera multiple } \\
\text { nucleopolyhedrovirus }\end{array}$ & $\begin{array}{l}\text { gi215401390- } \\
\text { NC_011615 }\end{array}$ & $\begin{array}{l}\text { occlusion derived viral } \\
\text { protein-e } 27\end{array}$ & 30.68 & $\mathrm{x}$ \\
\hline $\begin{array}{l}\text { Helicoverpa armigera multiple } \\
\text { nucleopolyhedrovirus }\end{array}$ & $\begin{array}{l}\text { gi215401289- } \\
\text { NC_011615 }\end{array}$ & superoxide dismutase & 32.48 & $\mathrm{x}$ \\
\hline Heliothis virescens ascovirus $3 \mathrm{e}$ & $\begin{array}{l}\text { gi134287217- } \\
\text { NC_009233 }\end{array}$ & hypothetical protein & 49.09 & $\mathrm{x}$ \\
\hline Heterosigma akashiwo RNA virus & $\begin{array}{l}\text { gi38707889- } \\
\text { NC_005281 }\end{array}$ & polyprotein & 34.25 & $\mathrm{x}$ \\
\hline Kakugo virus & $\begin{array}{l}\text { gi47177089- } \\
\text { NC_005876 }\end{array}$ & polyprotein & 26.14 & $\mathrm{x}$ \\
\hline Listeria phage A511 & $\begin{array}{l}\text { gi56693158- } \\
\text { NC_006565 }\end{array}$ & gp85 & 31.34 & \\
\hline Marine RNA virus JP-A & $\begin{array}{l}\text { gi157325040- } \\
\text { NC_009811 }\end{array}$ & hypothetical protein & 35.91 & $\mathrm{x}$ \\
\hline Marine RNA virus JP-A & $\begin{array}{l}\text { gi156563986- } \\
\text { NC_009757 }\end{array}$ & hypothetical protein & 27.35 & $\mathrm{x}$ \\
\hline Marine RNA virus JP-A & $\begin{array}{l}\text { gi156563986- } \\
\text { NC_009757 }\end{array}$ & hypothetical protein & 27.95 & $\mathrm{x}$ \\
\hline Marine RNA virus JP-A & $\begin{array}{l}\text { gi156563985- } \\
\text { NC_009757 }\end{array}$ & hypothetical protein & 32.6 & $\mathrm{x}$ \\
\hline
\end{tabular}


Table 2. (cont.) RNA viral metatranscriptomic hits to proteins predicted from known RNA viral genomes

\begin{tabular}{|c|c|c|c|c|}
\hline Species & Accession \# & Annotation & $\begin{array}{l}\text { Percent } \\
\text { Identity }\end{array}$ & $\begin{array}{c}\text { GOS } \\
\text { core } \\
\text { cluster }\end{array}$ \\
\hline Marine RNA virus JP-A & $\begin{array}{l}\text { gi156563986- } \\
\text { NC_009757 }\end{array}$ & hypothetical protein & 35.91 & $\mathrm{x}$ \\
\hline Marine RNA virus JP-A & $\begin{array}{l}\text { gi156563986- } \\
\text { NC_009757 }\end{array}$ & hypothetical protein & 27.35 & $\mathrm{x}$ \\
\hline Marine RNA virus JP-A & $\begin{array}{l}\text { gi156563986- } \\
\text { NC_009757 }\end{array}$ & hypothetical protein & 27.95 & $\mathrm{x}$ \\
\hline Marine RNA virus JP-A & $\begin{array}{l}\text { gi156563985- } \\
\text { NC_009757 }\end{array}$ & hypothetical protein & 32.6 & $\mathrm{x}$ \\
\hline Marine RNA virus JP-A & $\begin{array}{l}\text { gi156563986- } \\
\text { NC_009757 }\end{array}$ & hypothetical protein & 34.48 & $\mathrm{x}$ \\
\hline Marine RNA virus JP-A & $\begin{array}{l}\text { gi } 156563985- \\
\text { NC_009757 }\end{array}$ & hypothetical protein & 29.6 & $\mathrm{x}$ \\
\hline Marine RNA virus JP-A & $\begin{array}{l}\text { gi156563985- } \\
\text { NC_009757 }\end{array}$ & hypothetical protein & 39.93 & $\mathrm{x}$ \\
\hline Marine RNA virus JP-A & $\begin{array}{l}\text { gi } 156563985- \\
\text { NC_009757 }\end{array}$ & hypothetical protein & 46.67 & $\mathrm{x}$ \\
\hline Marine RNA virus JP-A & $\begin{array}{l}\text { gi156563986- } \\
\text { NC_009757 }\end{array}$ & hypothetical protein & 33.66 & $\mathrm{x}$ \\
\hline Marine RNA virus JP-B & $\begin{array}{l}\text { gi156563986- } \\
\text { NC_009757 }\end{array}$ & hypothetical protein & 26.23 & $\mathrm{x}$ \\
\hline Marine RNA virus JP-B & $\begin{array}{l}\text { gi156563988- } \\
\text { NC_009758 }\end{array}$ & hypothetical protein & 31.51 & $\mathrm{x}$ \\
\hline Marine RNA virus JP-B & $\begin{array}{l}\text { gi156563989- } \\
\text { NC_009758 }\end{array}$ & hypothetical protein & 33.59 & $\mathrm{x}$ \\
\hline $\begin{array}{l}\text { Paramecium bursaria Chlorella virus } \\
\text { FR483 }\end{array}$ & $\begin{array}{l}\text { gi156563988- } \\
\text { NC_009758 }\end{array}$ & N779R hypothetical protein & 29.63 & $\mathrm{x}$ \\
\hline Pelagibacter phage HTVC008M & $\begin{array}{l}\text { gi155370877- } \\
\text { NC_008603 }\end{array}$ & prohead core protein & 39.39 & \\
\hline
\end{tabular}


Table 2. (cont.) RNA viral metatranscriptomic hits to proteins predicted from known RNA viral genomes

\begin{tabular}{|c|c|c|c|c|}
\hline Species & Accession \# & Annotation & $\begin{array}{l}\text { Percent } \\
\text { Identity }\end{array}$ & $\begin{array}{l}\text { GOS core } \\
\text { cluster }\end{array}$ \\
\hline Pelagibacter phage HTVC008M & $\begin{array}{l}\text { gi448936879- } \\
\text { KC465899 }\end{array}$ & major capsid & 33.48 & \\
\hline Perina nuda virus & $\begin{array}{l}\text { gi448936880- } \\
\text { KC465899 }\end{array}$ & polyprotein & 21.01 & \\
\hline Phytophthora infestans RNA virus 1 & $\begin{array}{l}\text { gi15741226- } \\
\text { NC_003113 }\end{array}$ & hypothetical protein & 26.71 & \\
\hline Phytophthora infestans RNA virus 1 & $\begin{array}{l}\text { gi270358331- } \\
\text { NC_013221 }\end{array}$ & hypothetical protein & 22.52 & \\
\hline Phytophthora infestans RNA virus 1 & $\begin{array}{l}\text { gi270358331- } \\
\text { NC_013221 }\end{array}$ & hypothetical protein & 26.99 & \\
\hline Phytophthora infestans RNA virus 1 & $\begin{array}{l}\text { gi270358331- } \\
\text { NC_013221 }\end{array}$ & hypothetical protein & 32.28 & \\
\hline Phytophthora infestans RNA virus 1 & $\begin{array}{l}\text { gi270358331- } \\
\text { NC_013221 }\end{array}$ & hypothetical protein & 30.57 & \\
\hline Phytophthora infestans RNA virus 1 & $\begin{array}{l}\text { gi270358329- } \\
\text { NC_013220 }\end{array}$ & hypothetical protein & 42.28 & \\
\hline Phytophthora infestans RNA virus 1 & $\begin{array}{l}\text { gi270358329- } \\
\text { NC_013220 }\end{array}$ & hypothetical protein & 31.62 & \\
\hline Phytophthora infestans RNA virus 1 & $\begin{array}{l}\text { gi270358331- } \\
\text { NC_013221 }\end{array}$ & hypothetical protein & 26.79 & \\
\hline Phytophthora infestans RNA virus 1 & $\begin{array}{l}\text { gi270358331- } \\
\text { NC_013221 }\end{array}$ & hypothetical protein & 24.27 & \\
\hline Phytophthora infestans RNA virus 1 & $\begin{array}{l}\text { gi270358331- } \\
\text { NC_013221 }\end{array}$ & hypothetical protein & 53.33 & \\
\hline Phytophthora infestans RNA virus 1 & $\begin{array}{l}\text { gi270358331- } \\
\text { NC_013221 }\end{array}$ & hypothetical protein & 24.89 & \\
\hline Phytophthora infestans RNA virus 1 & $\begin{array}{l}\text { gi270358331- } \\
\text { NC_013221 }\end{array}$ & hypothetical protein & 24.91 & \\
\hline
\end{tabular}


Table 2. (cont.) RNA viral metatranscriptomic hits to proteins predicted from known RNA viral genomes

\begin{tabular}{|c|c|c|c|c|}
\hline Species & Accession \# & Annotation & $\begin{array}{l}\text { Percent } \\
\text { Identity }\end{array}$ & $\begin{array}{l}\text { GOS core } \\
\text { cluster }\end{array}$ \\
\hline Phytophthora infestans RNA virus 1 & $\begin{array}{l}\text { gi270358331- } \\
\text { NC_013221 }\end{array}$ & hypothetical protein & 32.87 & \\
\hline Phytophthora infestans RNA virus 1 & $\begin{array}{l}\text { gi270358329- } \\
\text { NC_013220 }\end{array}$ & hypothetical protein & 28.47 & \\
\hline Phytophthora infestans RNA virus 1 & $\begin{array}{l}\text { gi270358331- } \\
\text { NC_013221 }\end{array}$ & hypothetical protein & 22.92 & \\
\hline Phytophthora infestans RNA virus 1 & $\begin{array}{l}\text { gi270358329- } \\
\text { NC_013220 }\end{array}$ & hypothetical protein & 44.53 & \\
\hline Phytophthora infestans RNA virus 1 & $\begin{array}{l}\text { gi270358329- } \\
\text { NC_013220 }\end{array}$ & hypothetical protein & 42.48 & \\
\hline Phytophthora infestans RNA virus 1 & $\begin{array}{l}\text { gi270358331- } \\
\text { NC_013221 }\end{array}$ & hypothetical protein & 41.59 & \\
\hline Phytophthora infestans RNA virus 1 & $\begin{array}{l}\text { gi270358331- } \\
\text { NC_013221 }\end{array}$ & hypothetical protein & 23.77 & \\
\hline Phytophthora infestans RNA virus 1 & $\begin{array}{l}\text { gi270358329- } \\
\text { NC_013220 }\end{array}$ & hypothetical protein & 25.12 & \\
\hline Phytophthora infestans RNA virus 1 & $\begin{array}{l}\text { gi270358329- } \\
\text { NC_013220 }\end{array}$ & hypothetical protein & 34.76 & \\
\hline Phytophthora infestans RNA virus 1 & $\begin{array}{l}\text { gi270358329- } \\
\text { NC_013220 }\end{array}$ & hypothetical protein & 40.26 & \\
\hline Phytophthora infestans RNA virus 1 & $\begin{array}{l}\text { gi270358329- } \\
\text { NC_013220 }\end{array}$ & hypothetical protein & 35.15 & \\
\hline Phytophthora infestans RNA virus 1 & $\begin{array}{l}\text { gi270358329- } \\
\text { NC_013220 }\end{array}$ & hypothetical protein & 29.67 & \\
\hline Phytophthora infestans RNA virus 1 & $\begin{array}{l}\text { gi270358329- } \\
\text { NC_013220 }\end{array}$ & hypothetical protein & 41.14 & \\
\hline Phytophthora infestans RNA virus 1 & $\begin{array}{l}\text { gi270358329- } \\
\text { NC_013220 }\end{array}$ & hypothetical protein & 40.94 & \\
\hline
\end{tabular}




\begin{tabular}{|c|c|c|c|c|}
\hline Species & Accession \# & Annotation & $\begin{array}{l}\text { Percent } \\
\text { Identity }\end{array}$ & $\begin{array}{c}\text { GOS core } \\
\text { cluster }\end{array}$ \\
\hline Phytophthora infestans RNA virus 1 & $\begin{array}{l}\text { gi270358331- } \\
\text { NC_013221 }\end{array}$ & hypothetical protein & 27.85 & \\
\hline Phytophthora infestans RNA virus 1 & $\begin{array}{l}\text { gi270358331- } \\
\text { NC_013221 }\end{array}$ & hypothetical protein & 27.03 & \\
\hline Phytophthora infestans RNA virus 1 & $\begin{array}{l}\text { gi270358331- } \\
\text { NC_013221 }\end{array}$ & hypothetical protein & 24.73 & \\
\hline Phytophthora infestans RNA virus 1 & $\begin{array}{l}\text { gi270358329- } \\
\text { NC_013220 }\end{array}$ & hypothetical protein & 29.55 & \\
\hline Phytophthora infestans RNA virus 1 & $\begin{array}{l}\text { gi270358329- } \\
\text { NC_013220 }\end{array}$ & hypothetical protein & 41.47 & \\
\hline Phytophthora infestans RNA virus 1 & $\begin{array}{l}\text { gi270358329- } \\
\text { NC_013220 }\end{array}$ & hypothetical protein & 30.68 & \\
\hline Phytophthora infestans RNA virus 1 & $\begin{array}{l}\text { gi270358329- } \\
\text { NC_013220 }\end{array}$ & hypothetical protein & 35.62 & \\
\hline Phytophthora infestans RNA virus 1 & $\begin{array}{l}\text { gi270358329- } \\
\text { NC_013220 }\end{array}$ & hypothetical protein & 43.23 & \\
\hline Phytophthora infestans RNA virus 1 & $\begin{array}{l}\text { gi270358329- } \\
\text { NC_013220 }\end{array}$ & hypothetical protein & 26.16 & \\
\hline Phytophthora infestans RNA virus 1 & $\begin{array}{l}\text { gi270358331- } \\
\text { NC_013221 }\end{array}$ & hypothetical protein & 26.74 & \\
\hline Phytophthora infestans RNA virus 1 & $\begin{array}{l}\text { gi270358329- } \\
\text { NC_013220 }\end{array}$ & hypothetical protein & 34.11 & \\
\hline Phytophthora infestans RNA virus 1 & $\begin{array}{l}\text { gi270358329- } \\
\text { NC_013220 }\end{array}$ & hypothetical protein & 40 & \\
\hline Prochlorococcus phage P-SSM2 & $\begin{array}{l}\text { gi61806007- } \\
\text { NC_006883 }\end{array}$ & 23 major capsid protein & 43.18 & $\mathrm{x}$ \\
\hline Raphanus sativus cryptic virus 3 & $\begin{array}{l}\text { gi218148635- } \\
\text { NC_011705 }\end{array}$ & $\begin{array}{l}\text { putative RNA-dependent } \\
\text { RNA polymerase }\end{array}$ & 48.67 & $\mathrm{x}$ \\
\hline
\end{tabular}


Table 2. (cont.) RNA viral metatranscriptomic hits to proteins predicted from known RNA viral genomes

\begin{tabular}{|c|c|c|c|c|}
\hline Species & Accession \# & Annotation & $\begin{array}{l}\text { Percent } \\
\text { Identity }\end{array}$ & $\begin{array}{l}\text { GOS } \\
\text { core } \\
\text { cluster }\end{array}$ \\
\hline Raphanus sativus cryptic virus 3 & $\begin{array}{l}\text { gi218148635- } \\
\text { NC_011705 }\end{array}$ & $\begin{array}{l}\text { putative RNA-dependent RNA } \\
\text { polymerase }\end{array}$ & 35.14 & $\mathrm{x}$ \\
\hline Rice grassy stunt virus & $\begin{array}{l}\text { gi9635230- } \\
\text { NC_002323 }\end{array}$ & RNA polymerase & 25 & $\mathrm{x}$ \\
\hline Rice grassy stunt virus & $\begin{array}{l}\text { gi9635230- } \\
\text { NC_002323 }\end{array}$ & RNA polymerase & 25.81 & $\mathrm{x}$ \\
\hline $\begin{array}{l}\text { Rosellinia necatrix megabirnavirus } \\
\text { 1/W779 }\end{array}$ & $\begin{array}{l}\text { gi262396915- } \\
\text { NC_013462 }\end{array}$ & $\begin{array}{l}\text { RNA-dependent RNA } \\
\text { polymerase }\end{array}$ & 30.33 & $\mathrm{x}$ \\
\hline Satsuma dwarf virus & $\begin{array}{l}\text { gi20451009- } \\
\text { NC_003785 }\end{array}$ & polyprotein & 28.95 & $\mathrm{x}$ \\
\hline Synechococcus phage S-RSM4 & $\begin{array}{l}\text { gi255929159- } \\
\text { NC_013085 }\end{array}$ & ssDNA binding protein & 57.32 & $\mathrm{x}$ \\
\hline Uukuniemi virus & $\begin{array}{l}\text { gi38371702- } \\
\text { NC_005214 }\end{array}$ & RNA polymerase & 22.92 & $\mathrm{x}$ \\
\hline Uukuniemi virus & $\begin{array}{l}\text { gi38371708- } \\
\text { NC_005221 }\end{array}$ & hypothetical protein & 32.14 & $\mathrm{x}$ \\
\hline Varroa destructor virus-1 & $\begin{array}{l}\text { gi56121876- } \\
\text { NC_006494 }\end{array}$ & polyprotein & 22.41 & $\mathrm{x}$ \\
\hline Wheat eqlid mosaic virus & $\begin{array}{l}\text { gi157265284- } \\
\text { NC_009805 }\end{array}$ & polyprotein & 31.46 & $\mathrm{x}$ \\
\hline
\end{tabular}


Table 3. Reduction of data from feature detection and annotation in MAVEN to the final dissolved lipidome and manual functional group annotation.

\begin{tabular}{lrc}
\hline & $\begin{array}{c}\text { Number of } \\
\text { Features }\end{array}$ & $\begin{array}{c}\text { Average Peak } \\
\text { Area }\end{array}$ \\
\hline Features detected in MAVEN & 4460 & \\
Features annotated in MAVEN & 2030 & \\
Annotations adhering to "Adduct Hierarchy Rules" & 1273 & $9.96 \times 10^{8}$ \\
"High Confidence" annotations & 987 & $9.67 \times 10^{8}$ \\
Free fatty acids and oxylipins & 372 & $9.67 \times 10^{8}$ \\
Even number of carbons & 361 & $8.60 \times 10^{8}$ \\
Broad RT criteria met (1-18min) & 265 & $8.59 \times 10^{8}$ \\
$\quad$ Functional group annotation & 31 & $5.06 \times 10^{8}$ \\
$\quad$ Verified by authentic standard & 11 & $1.59 \times 10^{8}$
\end{tabular}




\begin{tabular}{|c|c|c|c|c|c|}
\hline Standard & $\begin{array}{l}\text { Structural } \\
\text { annotation }\end{array}$ & $\mathrm{RT}_{\text {standard }}$ & $\log \mathrm{P}$ & $\mathrm{RT}_{(\log \mathrm{P})}$ & $\Delta \mathrm{RT} \min$ \\
\hline 15-HEPE & NVO C20:5 10 & 4.56 & 4.48 & 4.41 & 0.15 \\
\hline 15-KETE & NVO C20:5 10 & 5.24 & 5.08 & 5.26 & -0.02 \\
\hline 12-KETE & NVO C20:5 10 & 5.63 & 5.32 & 5.60 & 0.03 \\
\hline 14,15 ЕpETE & NVO C20:5 10 & 5.41 & 4.7 & 4.73 & 0.68 \\
\hline 5-HрETE & NVO C20:4 20 & 5.01 & 5.56 & 5.95 & -0.94 \\
\hline 12-НpЕTЕ & NVO C20:4 20 & 5.95 & 5.8 & 6.29 & -0.34 \\
\hline 15-НpETE & NVO C20:4 20 & 5.73 & 5.04 & 5.21 & 0.52 \\
\hline 5,6 diHETE & NVO C20:4 20 & 3.48 & 3.59 & 3.15 & 0.33 \\
\hline 14,15 diHETE & NVO C20:4 20 & 3 & 3.59 & 3.15 & -0.15 \\
\hline 15-HETE & NVO C20:4 10 & 5.43 & 5.26 & 5.56 & -0.13 \\
\hline 15-HETrE & NVO C $20: 310$ & 6.11 & 5.99 & 6.78 & -0.67 \\
\hline
\end{tabular}

Table 4B. Functional group level annotation of the C20 oxylipin observed in dissolved lipidome. Retention times of authentic C20 standards presented in Table 4A were compared to the observed retention times of structural isomers observed in the DYEatom lipidome. Putative functional group annotations were assigned to features that had $\mathrm{RT}_{\mathrm{obs}}$ within 0.7 min of $\mathrm{RT}_{\text {standard }}$ for $-2 \mathrm{O}$ oxylipin and $0.5 \mathrm{~min}$ of $\mathrm{RT}_{\text {standard }}$ for $-1 \mathrm{O}$ oxylipins. $\mathrm{RT}_{\text {standard1-5 }}$ corresponded to the various standards with overlapping structural annotations. Abbreviations for oxylipins can be found in Appendix A.

\begin{tabular}{lccccccccccccc}
\hline NVO 20:3 1O RT-5.5 & 5.5 & 6.1 & -0.6 & & & & & & & & & \\
NVO 20:3 1O RT-6.5 & 6.5 & 6.1 & $\mathbf{0 . 5}$ & & & & & & & & & HETrE RT-6.5 2b \\
NVO 20:3 1O RT-7.3 & 7.3 & 6.1 & 1.2 & & & & & & & & & \\
NVO 20:4 1O RT-5.5 & 5.5 & 5.4 & $\mathbf{0 . 1}$ & & & & & & & & & HETE RT-5.5 2b \\
NVO 20:4 1O RT-6.7 & 6.7 & 5.4 & 1.3 & & & & & & & & & \\
NVO 20:4 2O RT-2.3 & 2.3 & 5.0 & -2.7 & 6.0 & -3.7 & 5.7 & -3.4 & 3.5 & -1.2 & 3.0 & -0.7 & \\
NVO 20:4 2O RT-3.7 & 3.7 & 5.0 & -1.3 & 6.0 & -2.3 & 5.7 & -2.0 & 3.5 & $\mathbf{0 . 2}$ & 3.0 & 0.7 & diHETE RT-3.7 2b \\
NVO 20:4 2O RT-5.9 & 5.9 & 5.0 & 0.9 & 6.0 & $\mathbf{- 0 . 1}$ & 5.7 & $\mathbf{0 . 2}$ & 3.5 & 2.4 & 3.0 & 2.9 & HpETE RT-5.9 2b \\
NVO 20:4 2O RT-7.3 & 7.3 & 5.0 & 2.3 & 6.0 & 1.3 & 5.7 & 1.6 & 3.5 & 3.8 & 3.0 & 4.3 & \\
NVO 20:4 2O RT-8.2 & 8.2 & 5.0 & 3.2 & 6.0 & 1.0 & 5.7 & 2.5 & 3.5 & 4.7 & 3.0 & 5.2 & \\
NVO 20:5 1O RT-4.6 & 4.6 & 4.6 & $\mathbf{0}$ & 5.2 & -0.6 & 5.6 & -1.0 & & & & & HEPE RT-4.6 2a \\
NVO 20:5 1O RT-5.9 & 5.9 & 4.6 & 1.3 & 5.2 & 0.7 & 5.6 & $\mathbf{0 . 3}$ & & & & & KETE RT-5.9 2b
\end{tabular}


Table 5A. Retention times $\left(\mathrm{RT}_{\text {standard }}\right)$ and octanol water partition coefficients $(\log \mathrm{P})$ for the standard C18:n oxylipins. Linear regression analysis gave Equation 4 which was used to calculate theoretical RT for the standards $\left(\mathrm{RT}_{\log \mathrm{P}}\right)$.

(Eq. 4) $R T_{\log P}=\frac{\log P-1.0479}{0.909}\left(\mathrm{R}^{2}=0.99 ; \mathrm{F}=0.0001\right)$

\begin{tabular}{llcccc}
\hline $\begin{array}{l}\text { Standard } \\
\text { Name }\end{array}$ & Structural annotation & $\mathrm{RT}_{\text {standard }}$ & $\operatorname{logP}$ & $\mathrm{RT}_{\log P}$ & $\begin{array}{c}\Delta \mathrm{RT} \\
\mathrm{min}\end{array}$ \\
\hline 9-HpODE & NVO C18:2 2O & 5.44 & 6 & 5.45 & -0.01 \\
13-HpODE & NVO C18:2 2O & 5.44 & 6 & 5.45 & -0.01 \\
9-HOTrE & NVO C18:3 1O & 4.02 & 4.69 & 4.01 & 0.01 \\
13-HpOTrE & NVO C18:3 2O & 4.65 & 5.22 & 4.59 & 0.06 \\
9-HpOTrE & NVO C18:3 2O & 4.28 & 4.99 & 4.34 & -0.06 \\
9-KOTrE & NVO C18:4 1O & 4.10 & 5.92 & 5.36 & -1.26
\end{tabular}

Table 5B. Functional group level annotation of the C18:n oxylipins observed in dissolved lipidome. Retention times of authentic C18:n standards $\left(\mathrm{RT}_{\text {standard1-2 }}\right)$ presented in Table 5A were compared to the observed retention times of structural isomers $\left(\mathrm{RT}_{\mathrm{obs}}\right)$ in the DYEatom lipidome. Putative functional group annotations were assigned to features that had $\mathrm{RT}_{\text {obs }}$ within 0.7 min of $\mathrm{RT}_{\text {standard }}$ for $-2 \mathrm{O}$ oxylipin and $0.5 \mathrm{~min}$ of $\mathrm{RT}_{\text {standard }}$ for $-1 \mathrm{O}$ oxylipins. $\mathrm{RT}_{\text {standard } 1-2}$ corresponded to the various standards with overlapping structural annotations. Abbreviations for oxylipins can be found in Appendix A.

\begin{tabular}{|c|c|c|c|c|c|c|}
\hline \multirow[t]{2}{*}{ Structural annotation } & \multirow[b]{2}{*}{$\mathrm{RT}_{\mathrm{obs}}$} & \multirow[b]{2}{*}{$\mathrm{RT}_{\text {standard1 }}$} & \multirow[b]{2}{*}{$\Delta \mathrm{RT} \min$} & \multirow[b]{2}{*}{$\mathrm{RT}_{\text {standard2 }}$} & \multirow[b]{2}{*}{$\Delta \mathrm{RT} \min$} & \multirow[t]{2}{*}{$\begin{array}{l}\text { Proposed functional } \\
\text { group annotation }\end{array}$} \\
\hline & & & & & & \\
\hline NVO 18:2 20 & 3.2 & 5.4 & -2.2 & & & \\
\hline NVO 18:2 20 & 7.5 & 5.4 & 2.1 & & & \\
\hline NVO 18:2 20 & 5.7 & 5.4 & 0.3 & & & HpODE RT-5.7 2b \\
\hline NVO 18:2 20 & 4.5 & 5.4 & -0.9 & & & \\
\hline NVO $18: 310$ & 5.7 & 4.0 & 1.7 & & & \\
\hline NVO $18: 310$ & 4.3 & 4.0 & 0.3 & & & HOTrE RT-4.3 2b \\
\hline NVO 18:3 20 & 1.8 & 4.7 & -2.9 & 4.3 & -2.5 & \\
\hline NVO 18:3 20 & 3.5 & 4.7 & -1.2 & 4.3 & -0.8 & \\
\hline NVO 18:3 20 & 2.5 & 4.7 & -2.2 & 4.3 & -1.8 & \\
\hline NVO 18:3 20 & 5.4 & 4.7 & 0.7 & 4.3 & 1.1 & HpOTrE RT-5.4 2b \\
\hline NVO 18:3 20 & 6.8 & 4.7 & 2.1 & 4.3 & 2.5 & \\
\hline NVO $18: 320$ & 7.9 & 4.7 & 3.2 & 4.3 & 3.6 & \\
\hline NVO 18:3 20 & 4.6 & 4.7 & -0.1 & 4.3 & 0.3 & HpOTrE RT-4.6 2b \\
\hline NVO 18:4 10 & 4.6 & 4.1 & 0.5 & & & KOTrE RT-4.6 2b \\
\hline NVO $18: 410$ & 3.3 & 4.1 & -0.8 & & & \\
\hline
\end{tabular}


Table 6. The retention time of the $\mathrm{C} 20: 4$ standards were used to predict theoretical retention times for $\mathrm{C} 20: 5$ analogs $\left(\mathrm{RT}_{+\mathrm{DB}}\right)$ using the offset of 0.87 minutes between 15-HETE (5.43) and 15-HEPE (4.56).

\begin{tabular}{|c|c|c|c|c|c|}
\hline $\begin{array}{l}\text { C20:4 Standard } \\
\text { Functional Group } \\
\text { Annotation }\end{array}$ & $\begin{array}{c}\text { C18:n Standard } \\
\text { Structural } \\
\text { Annotation }\end{array}$ & $\mathrm{RT}_{\mathrm{obs}}$ & $\begin{array}{l}\text { C20:5Analog } \\
\text { Functional } \\
\text { Group } \\
\text { Annotation }\end{array}$ & $\begin{array}{l}\text { C20:5 Analog } \\
\text { Structural } \\
\text { Annotation }\end{array}$ & $\mathrm{RT}_{+\mathrm{DB}}$ \\
\hline 5-НpETE & NVO C20:4 20 & 5.01 & HpEPE & NVO C20:5 20 & 4.14 \\
\hline 12-HрETE & NVO C20:4 20 & 5.95 & HpEPE & NVO C20:5 20 & 5.08 \\
\hline 15-НpETE & NVO C20:4 20 & 5.73 & HpEPE & NVO C20:5 20 & 4.86 \\
\hline 5,6 diHETE & NVO C20:4 20 & 3.48 & diHEPE & NVO C20:5 20 & 2.61 \\
\hline 14,15 diHETE & NVO C20:4 20 & 3.0 & diHEPE & NVO C20:5 20 & 2.13 \\
\hline 15-HETE & NVO C20:4 10 & 5.43 & HEPE & NVO C20:5 10 & 4.56 \\
\hline
\end{tabular}


Table 7. Functional group level annotation of the C20:5 oxylipins observed in dissolved lipidome. Retention times of authentic C20 standards (Table 4A) were used to calculate theoretical retention times $\left(\mathrm{RT}_{+\mathrm{DB}}\right)$ of $\mathrm{C} 20: 5$ structural isomers based on the $\sim 0.9$ minute offset observed for 15-HEPE (4.56) and 15-HETE (5.43). Putative functional group annotations were assigned to features that had $\mathrm{RT}_{\mathrm{obs}}$ within 0.7 min of $\mathrm{RT}_{+\mathrm{DB}}$ for $-2 \mathrm{O}$ oxylipin and $0.5 \mathrm{~min}$ of $\mathrm{RT}_{+\mathrm{DB}}$ for $-1 \mathrm{O}$ oxylipins. $\mathrm{RT}_{\mathrm{DB} 1-\mathrm{DB} 3}$ corresponded to $\mathrm{RT}$ predicted from various standards with overlapping structural annotations. Asterisk denotes verification by authentic standard. Abbreviations for oxylipins can be found in Appendix A.

\begin{tabular}{|c|c|c|c|c|c|c|c|c|}
\hline Structural level annotation & $\mathrm{RT}_{\mathrm{obs}}$ & $\mathrm{RT}_{+\mathrm{DB} 1}$ & $\Delta \mathrm{RT} \min$ & $\mathrm{RT}_{+\mathrm{DB} 2}$ & $\Delta \mathrm{RT} \min$ & $\mathrm{RT}_{+\mathrm{DB} 3}$ & $\Delta \mathrm{RT} \min$ & $\begin{array}{c}\text { Putative } \\
\text { functional group } \\
\text { annotation }\end{array}$ \\
\hline NVO C20:5 2O RT-2.1 & 2.1 & 2.1 & $\mathbf{0}$ & 2.6 & -0.5 & & & diHEPE RT-2.1 3a \\
\hline NVO C20:5 2O RT-2.9 & 2.9 & 2.1 & 0.8 & 2.6 & 0.3 & & & diHEPE RT-2.9 3b \\
\hline NVO C20:5 2O RT-4.9 & 4.9 & 4.1 & 0.8 & 4.9 & $\mathbf{0}$ & 5.1 & -0.2 & HpEPE RT-4.9 3a \\
\hline NVO C20:5 2O RT-6.7 & 6.7 & 4.1 & 2.6 & 4.9 & 1.8 & 5.1 & 1.6 & \\
\hline NVO C20:5 2O RT-7.5 & 7.5 & 4.1 & 3.4 & 4.9 & 2.6 & 5.1 & 2.4 & \\
\hline NVO C20:5 1O RT-4.6 & 4.6 & 4.6 & $\mathbf{0}$ & & & & & HEPE RT-4.6* $3 \mathrm{a}$ \\
\hline NVO C20:5 1O RT-5.9 & 5.9 & 4.6 & 2.3 & & & & & \\
\hline NVO C20:5 1O RT-7.1 & 7.1 & 4.6 & 2.5 & & & & & \\
\hline
\end{tabular}


Table 8. The retention time of the C18:n standards were used to predict theoretical retention times for C16:n analogs $\left(\mathrm{RT}_{\mathrm{C} 18->\mathrm{C} 16}\right)$ using the offset of 2.09 minutes between 15-HETrE (6.11) and 9-HOTrE (4.02). Theoretical retention times were also calculated for less unsaturated $\left(\mathrm{RT}_{-\mathrm{DB}}\right.$ and $\left.\mathrm{RT}_{-2 \mathrm{DB}}\right)$ and more unsaturated $\left(\mathrm{RT}_{+\mathrm{DB}}\right) \mathrm{C} 16: \mathrm{n}$ molecules using the offset of 0.79 minutes observed for HpODE (5.44) and HpOTrE (4.65).

\begin{tabular}{|c|c|c|c|c|c|c|c|c|}
\hline $\begin{array}{l}\text { C18:n } \\
\text { Standard } \\
\text { Functional } \\
\text { Group } \\
\text { Annotation }\end{array}$ & $\begin{array}{c}\text { C18:n Standard } \\
\text { Structural } \\
\text { Annotation }\end{array}$ & $\mathrm{RT}_{\mathrm{obs}}$ & $\begin{array}{c}\text { C16:n } \\
\text { Analog } \\
\text { Functional } \\
\text { Group } \\
\text { Annotation }\end{array}$ & $\begin{array}{l}\text { C16:n Analog } \\
\text { Structural } \\
\text { Annotation }\end{array}$ & $\mathrm{RT}_{\mathrm{C} 18->\mathrm{C} 16}$ & $\begin{array}{l}\text { C16:n-1 } \\
\text { Analog } \\
\text { Functional } \\
\text { Group } \\
\text { Annotation }\end{array}$ & $\begin{array}{c}\text { C16:n-1 } \\
\text { Analog } \\
\text { Structural } \\
\text { Annotation }\end{array}$ & $\mathrm{RT}_{-\mathrm{DB}}$ \\
\hline HpODE & NVO C18:2 20 & 5.44 & HpHDE & NVO 16:2 20 & 3.35 & НpHME & NVO 16:120 & 4.14 \\
\hline HOTrE & NVO C18:3 10 & 4.02 & HHTrE & NVO $16: 310$ & 1.93 & HHDE & NVO 16:2 10 & 2.72 \\
\hline HpOTrE & NVO C18:3 20 & 4.65 & HpHTrE & NVO $16: 320$ & 2.56 & HpHDE & NVO $16: 220$ & 3.35 \\
\hline HpOTrE & NVO C18:3 20 & 4.28 & HpHTrE & NVO $16: 320$ & 2.19 & HpHDE & NVO 16:2 20 & 2.98 \\
\hline KOTrE & NVO C18:4 10 & 4.10 & KHTrE & NVO $16: 410$ & 2.01 & KHDE & NVO $16: 310$ & 2.80 \\
\hline
\end{tabular}

\begin{tabular}{|c|c|c|c|c|c|c|c|c|}
\hline $\begin{array}{c}\text { C18:n } \\
\text { Standard } \\
\text { Functional } \\
\text { Group } \\
\text { Annotation }\end{array}$ & $\begin{array}{l}\text { C18:n Standard } \\
\text { Structural } \\
\text { Annotation }\end{array}$ & $\mathrm{RT}_{\mathrm{obs}}$ & $\begin{array}{l}\text { C16:n+1 } \\
\text { Analog } \\
\text { Functional } \\
\text { Group } \\
\text { Annotation }\end{array}$ & $\begin{array}{c}\text { C16:n+1 } \\
\text { Analog } \\
\text { Structural } \\
\text { Annotation }\end{array}$ & $\mathrm{RT}_{+\mathrm{DB}}$ & $\begin{array}{l}\text { C16:n-2 } \\
\text { Analog } \\
\text { Functional } \\
\text { Group } \\
\text { Annotation }\end{array}$ & $\begin{array}{c}\text { C16:n-2 } \\
\text { Analog } \\
\text { Structural } \\
\text { Annotation }\end{array}$ & $\mathrm{RT}_{-2 \mathrm{DB}}$ \\
\hline HpODE & NVO C18:2 20 & 5.44 & HpHTrE & NVO 16:32O & 3.27 & & & \\
\hline HOTrE & NVO C18:310 & 4.02 & HHTE & NVO 16:4 10 & 1.85 & HHME & NVO 16:1 10 & 3.51 \\
\hline HpOTrE & NVO C18:3 20 & 4.65 & HpHTE & NVO 16:4 20 & 2.48 & HрHME & NVO 16:1 20 & 4.14 \\
\hline HpOTrE & NVO C18:3 20 & 4.28 & HpHTE & NVO 16:4 20 & 2.11 & HpHME & NVO 16:1 20 & 3.77 \\
\hline KOTrE & NVO C18:4 10 & 4.10 & KHTE & NVO 16:5 10 & 5.36 & KHME & NVO 16:2 10 & 3.59 \\
\hline
\end{tabular}


Table 9. Functional group level annotation of C16:n analogs of C18:n standards. Retention times observed for 16:n structural isomers were compared to the theoretical retention times calculated from the RT of C18:n standards using the 2.09 min offset observed for the RT of 15-HETrE (6.11) and 9-HOTrE (4.02) (Table 8). Putative functional group annotations were assigned to features that had $\mathrm{RT}_{\text {obs }}$ within 0.7 min of $\mathrm{RT}_{(\mathrm{C} 18->\mathrm{C} 18)}$ for $-2 \mathrm{O}$ oxylipin and 0.5 min of $\mathrm{RT}\left({ }_{\mathrm{C} 18->\mathrm{C} 18)}\right.$ for $-1 \mathrm{O}$ oxylipins. $\mathrm{RT}_{(\mathrm{C} 18->\mathrm{C} 18) 1-2}$ corresponded to $\mathrm{RT}$ predicted from various standards with overlapping structural annotations. An asterisk denotes corroborating annotation between theoretical RT presented here and those in Table 10A. A carat denotes competing annotation with Table 10B. Abbreviations for oxylipins can be found in Appendix A.

\begin{tabular}{|c|c|c|c|c|c|c|}
\hline $\begin{array}{c}\text { Structural annotation of } \\
\text { C16:n molecular species } \\
\text { in the DYEatom } \\
\text { lipidome }\end{array}$ & $\mathrm{RT}_{\mathrm{obs}}$ & $\mathrm{RT}_{(\mathrm{C} 18->\mathrm{C} 16) 1}$ & $\begin{array}{l}\Delta \mathrm{RT} \\
\min \end{array}$ & $\mathrm{RT}_{(\mathrm{C} 18->\mathrm{C} 16) 2}$ & $\begin{array}{l}\Delta \mathrm{RT} \\
\min \end{array}$ & $\begin{array}{l}\text { Functional group } \\
\text { annotation }\end{array}$ \\
\hline NVO C16:2 2O RT- 2 & 2.0 & 3.4 & -1.4 & & & \multirow{5}{*}{ HpHDE RT-3.4* (3a) } \\
\hline NVO C16:2 2O RT- 3.4 & 3.4 & 3.4 & 0 & & & \\
\hline NVO C16:3 1O RT- 2.4 & 2.4 & 1.9 & 0.5 & & & \\
\hline NVO C16:3 1O RT- 3.4 & 3.4 & 1.9 & 1.5 & & & \\
\hline NVO C16:3 2O RT- 1.8 & 1.8 & 2.6 & -0.8 & 2.2 & -0.4 & \\
\hline NVO C16:3 2O RT- 2.5 & 2.5 & 2.6 & -0.1 & 2.2 & 0.3 & \multirow[t]{2}{*}{ HpHTrE RT-2.5 (3b) } \\
\hline NVO C16:3 2O RT- 3.9 & 3.9 & 2.6 & 1.3 & 2.2 & 1.7 & \\
\hline NVO C16:4 1O RT- 2.1 & 2.1 & 2.0 & 0.1 & & & \multirow[t]{2}{*}{$\begin{array}{c}\mathrm{KHTrE} / \mathrm{HHTE}^{\wedge} \mathrm{RT}^{(3 \mathrm{~b})}-2.1 \\
\end{array}$} \\
\hline NVO C16:4 10 RT- 2.7 & 2.7 & 2.0 & 0.7 & & & \\
\hline
\end{tabular}


Table 10A. Functional group level annotation of 16:n-1 analogs of C18:n standards. Retention times observed for structural isomers $\left(\mathrm{RT}_{\text {obs }}\right)$ were compared to the theoretical $\mathrm{RT}$ values presented in Table 8. Putative functional group annotations were assigned to features that had $\mathrm{RT}_{\text {obs }}$ within 0.7 min of $\mathrm{RT}_{-\mathrm{DB}}$ for $-2 \mathrm{O}$ oxylipin and 0.5 min of $\mathrm{RT}_{-\mathrm{DB}}$ for $-1 \mathrm{O}$ oxylipins. Asterisk denotes verification by authentic standard. Abbreviations for oxylipins can be found in Appendix A.

\begin{tabular}{|c|c|c|c|c|c|c|c|}
\hline $\begin{array}{l}\text { C16:n-1 } \\
\text { Analog } \\
\text { Functional } \\
\text { Group } \\
\text { Annotation }\end{array}$ & $\begin{array}{c}\text { Structural } \\
\text { annotation of } \\
\text { molecular } \\
\text { species in } \\
\text { DYEatom } \\
\text { lipidome }\end{array}$ & $\mathrm{RT}_{\mathrm{obs}}$ & $\mathrm{RT}_{-\mathrm{DB}}$ & $\begin{array}{c}\Delta \mathrm{RT} \\
\min \end{array}$ & $\mathrm{RT}_{-\mathrm{DB}}$ & $\Delta \mathrm{RT} \min$ & $\begin{array}{c}\text { Functional group } \\
\text { annotation }\end{array}$ \\
\hline HpHME & NVO 16:1 20 & 4.6 & 4.1 & 0.5 & & & HpHME RT-4.6* (3b) \\
\hline HрHME & NVO 16:1 20 & 2.5 & 4.1 & -1.6 & & & \\
\hline HрHME & NVO 16:1 20 & 5.9 & 4.1 & 1.8 & & & \\
\hline HрHME & NVO 16:1 20 & 7.1 & 4.1 & 3.0 & & & \\
\hline HHDE & NVO 16:2 10 & 4.0 & 2.7 & 1.3 & & & \\
\hline HHDE & NVO 16:2 10 & 4.6 & 2.7 & 1.9 & & & \\
\hline HHDE & NVO 16:2 10 & 3.1 & 2.7 & 0.4 & & & HHDE RT-3.1 (3b) \\
\hline HHDE & NVO16:2 10 & 7.0 & 2.7 & 4.3 & & & \\
\hline HpHDE & NVO 16:2 20 & 2.0 & 3.4 & -1.4 & 3.0 & -1.0 & \\
\hline HpHDE & NVO 16:2 20 & 3.4 & 3.4 & $\mathbf{0}$ & 3.0 & 0.4 & HpHDE RT-3.4* (3a) \\
\hline KHDE & NVO 16:3 1O & 3.4 & 2.8 & 0.6 & & & \\
\hline KHDE & NVO 16:3 10 & 2.4 & 2.8 & -0.4 & & & KHDE RT-2.4 (3b) \\
\hline
\end{tabular}


Table 10B. Functional group level annotation of 16:n+1 analogs of C18:n standards. Retention times observed for structural isomers $\left(\mathrm{RT}_{\text {obs }}\right)$ were compared to the theoretical $\mathrm{RT}$ values presented in Table 8. Putative functional group annotations were assigned to features that had $\mathrm{RT}_{\text {obs }}$ within 0.7 min of $\mathrm{RT}_{+\mathrm{DB}}$ for $-2 \mathrm{O}$ oxylipin and $0.5 \mathrm{~min}$ of $\mathrm{RT}_{+\mathrm{DB}}$ for $-1 \mathrm{O}$ oxylipins. Asterisk denotes verification by authentic standard. A carat denotes competing annotation in Table 9. Abbreviations for oxylipins can be found in Appendix A.

\begin{tabular}{|c|c|c|c|c|c|c|c|}
\hline $\begin{array}{c}\text { C16:n+1 } \\
\text { Analog } \\
\text { Functional } \\
\text { Group } \\
\text { Annotation }\end{array}$ & $\begin{array}{l}\text { Structural } \\
\text { annotation of } \\
\text { molecular } \\
\text { species in } \\
\text { DYEatom } \\
\text { lipidome }\end{array}$ & $\mathrm{RT}_{\mathrm{obs}}$ & $\mathrm{RT}_{+\mathrm{DB}}$ & $\begin{array}{l}\Delta \mathrm{RT} \\
\min \end{array}$ & $\mathrm{RT}_{+\mathrm{DB}}$ & $\begin{array}{l}\Delta \mathrm{RT} \\
\min \end{array}$ & $\begin{array}{l}\text { Functional group } \\
\text { annotation }\end{array}$ \\
\hline HpHTrE & NVO 16:3 20 & 1.8 & 3.3 & -1.5 & & & \multirow{5}{*}{$\begin{array}{c}\text { HpHTrE RT-3.9 (3b) } \\
\text { KHTrE/HHTE^ RT-2.1 } \\
\text { (3b) }\end{array}$} \\
\hline HpHTrE & NVO 16:3 20 & 2.5 & 3.3 & 0.8 & & & \\
\hline HpHTrE & NVO 16:3 20 & 3.9 & 3.3 & 0.6 & & & \\
\hline HHTE & NVO 16:4 10 & 2.1 & 1.9 & 0.2 & & & \\
\hline HHTE & NVO 16:4 10 & 2.7 & 1.9 & 0.8 & & & \\
\hline НpHTE & NVO 16:4 20 & 1.7 & 2.5 & -1.1 & 2.1 & -0.4 & HpHTE RT-1.7 (3b) \\
\hline НpHTE & NVO 16:4 20 & 2.3 & 2.5 & -0.2 & 1.9 & 0.4 & HpHTE RT-2.3 (3b) \\
\hline KHTE & NVO 16:5 10 & & 1.9 & & & & \\
\hline
\end{tabular}

Table 10C. Functional group level annotation of 16:n-2 analogs of C18:n standards. Retention times observed for structural isomers $\left(\mathrm{RT}_{\mathrm{obs}}\right)$ were compared to the theoretical $\mathrm{RT}$ values presented in Table 8 . Putative functional group annotations were assigned to features that had $\mathrm{RT}_{\text {obs }}$ within 0.7 min of $\mathrm{RT}_{-2 \mathrm{DB}}$ for $-2 \mathrm{O}$ oxylipin and $0.5 \mathrm{~min}$ of $\mathrm{RT}_{-2 \mathrm{DB}}$ for $-1 \mathrm{O}$ oxylipins. Asterisk denotes verification by authentic standard.An asterisk denoted corroborating annotation between theoretical RT. Abbreviations for oxylipins can be found in Appendix A.

\begin{tabular}{|c|c|c|c|c|c|c|c|}
\hline $\begin{array}{l}\text { C16:n-2 } \\
\text { Analog } \\
\text { Functional } \\
\text { Group } \\
\text { Annotation }\end{array}$ & $\begin{array}{l}\text { Structural } \\
\text { annotation of } \\
\text { molecular } \\
\text { species in } \\
\text { DYEatom } \\
\text { lipidome }\end{array}$ & $\mathrm{RT}_{\mathrm{obs}}$ & $\mathrm{RT}_{-2 \mathrm{DB}}$ & $\Delta \mathrm{RT} \min$ & $\mathrm{RT}_{-2 \mathrm{DB}}$ & $\Delta \mathrm{RT} \min$ & $\begin{array}{l}\text { Functional group } \\
\text { annotation }\end{array}$ \\
\hline HHME & NVO 16:1 10 & 4.3 & 3.5 & 0.8 & & & \\
\hline HHME & NVO 16:1 10 & 7.2 & 3.5 & 3.7 & & & \\
\hline HрHME & NVO 16:1 20 & 4.6 & 4.1 & 0.5 & 3.8 & 0.8 & HpHME RT-4.6* (3b) \\
\hline HрHME & NVO 16:1 20 & 2.5 & 4.1 & -1.6 & 3.8 & -1.3 & \\
\hline HpHME & NVO 16:120 & 5.9 & 4.1 & 1.8 & 3.8 & 2.1 & \\
\hline HpHME & NVO 16:1 20 & 7.1 & 4.1 & 3.0 & 3.8 & 3.3 & \\
\hline KHME & NVO $16: 210$ & 4.0 & 3.6 & 0.4 & & & KHME RT-4 (3b) \\
\hline KHME & NVO 16:2 10 & 4.6 & 3.6 & 1.0 & & & \\
\hline KHME & NVO $16: 210$ & 3.1 & 3.6 & -0.5 & & & KHME RT-3.1 (3b) \\
\hline KHME & NVO 16:2 10 & 7.0 & 3.6 & 3.4 & & & \\
\hline
\end{tabular}


Table 11. Functional group level annotations of features annotated as PUAs within the dissolved lipidome. The retention times of the authentic standard decadienal $\left(\mathrm{RT}_{\text {auth }}\right)$ was used to predict retention times for structural isomers $\left(\mathrm{RT}_{\text {calc }}\right)$ for similar compounds in the dissolved lipidome. The difference in carbon number $(\mathrm{dC})$ and double bond number $(\mathrm{dDB})$ was calculated for the structural annotation of the observed feature and the structural annotation of the authentic

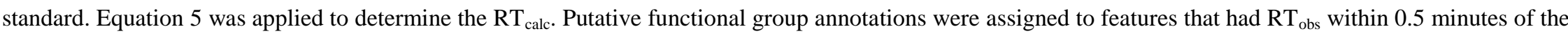
$\mathrm{RT}_{\text {calc }}$. Elemental formula annotations were assigned to all other features.

$$
\begin{aligned}
& \text { Equation ( 5) } R T_{\text {calc }}=R T_{\text {auth }}-\left(\# \text { carbons }_{\text {auth }}-\# \text { carbons }_{\text {obs }}\right) * \frac{1.05 \mathrm{~min}}{\text { carbon }} \\
& +\left(\# D B_{\text {auth }}-\# D B_{\text {obs }}\right) * \frac{0.9 \mathrm{~min}}{D B}
\end{aligned}
$$

\begin{tabular}{|c|c|c|c|c|c|c|c|c|c|c|}
\hline \multirow[t]{2}{*}{ Observed feature } & \multicolumn{4}{|c|}{ Authentic Standard } & \multicolumn{4}{|c|}{ Calculation } & \multirow{2}{*}{$\begin{array}{l}\text { Proposed } \\
\text { Functional Group } \\
\text { annotation }\end{array}$} & \multirow[b]{2}{*}{$\begin{array}{l}\text { Elemental formula } \\
\text { annotation }\end{array}$} \\
\hline & $\mathrm{RT}_{\mathrm{obs}}$ & $\begin{array}{l}\text { Structural } \\
\text { annotation }\end{array}$ & Standard & $\mathrm{RT}_{\text {auth }}$ & $\mathrm{dC}$ & $\mathrm{dDB}$ & $\mathrm{RT}_{\text {calc }}$ & $\mathrm{RT}_{\mathrm{obs}}-\mathrm{RT}_{\mathrm{calc}}$ & & \\
\hline PUA $10: 4$ none RT-1.7 & 1.7 & PUA 10:2 & Decadienal & 3.7 & 0 & 2 & 1.9 & -0.15 & decatetrienal (3b) & $\mathrm{C} 10 \mathrm{H} 12 \mathrm{O}$ \\
\hline PUA $10: 4$ none RT-3.2 & 3.2 & PUA 10:2 & Decadienal & 3.7 & 0 & 2 & 1.9 & 1.35 & & $\mathrm{C} 10 \mathrm{H} 12 \mathrm{O}$ \\
\hline PUA 10:3 none RT-4.9 & 4.9 & PUA 10:2 & Decadienal & 3.7 & 0 & 1 & 2.8 & 2.15 & & $\mathrm{C} 10 \mathrm{H} 14 \mathrm{O}$ \\
\hline PUA 10:2 none RT-1.5 & 1.5 & PUA 10:2 & Decadienal & 3.7 & 0 & 0 & 3.7 & -2.15 & & $\mathrm{C} 10 \mathrm{H} 16 \mathrm{O}$ \\
\hline PUA 10:2 none RT-4.9 & 4.9 & PUA 10:2 & Decadienal & 3.7 & 0 & 0 & 3.7 & 1.25 & & $\mathrm{C} 10 \mathrm{H} 16 \mathrm{O}$ \\
\hline PUA $12: 5$ none RT-4.9 & 4.9 & PUA 10:2 & Decadienal & 3.7 & 2 & 3 & 3.1 & 1.85 & & $\mathrm{C} 12 \mathrm{H} 14 \mathrm{O}$ \\
\hline PUA $12: 3$ none RT-4.9 & 4.9 & PUA 10:2 & Decadienal & 3.7 & 2 & 1 & 4.9 & 0.05 & dodecatrienal (3a) & \\
\hline
\end{tabular}


Table 12. Functional group level annotations of features annotated as C10 fatty acid within the dissolved lipidome. The retention times of the authentic standard octanoic acid $\left(\mathrm{RT}_{\text {auth }}\right)$ was used to predict retention times for structural isomers $\left(\mathrm{RT}_{\text {calc }}\right)$ for similar compounds in the dissolved lipidome. The difference in carbon number $(\mathrm{dC})$ and double bond number $(\mathrm{dDB})$ was calculated for the structural annotation of the observed feature and the structural annotation of the authentic standard. Equation 5 was applied to determine the $\mathrm{RT}_{\text {calc. }}$. Putative functional group annotations were assigned to features that had $\mathrm{RT}_{\text {obs }}$ within 0.5 minutes of the $\mathrm{RT}_{\text {calc. }}$. Elemental formula annotations were assigned to all other features.

Equation (5)

$$
\begin{aligned}
R T_{\text {calc }} & =R T_{\text {auth }}-\left(\# \text { carbons }_{\text {auth }}-\# \text { carbons }_{\text {obs }}\right) * \frac{1.05 \mathrm{~min}}{\text { carbon }} \\
& +\left(\# D B_{\text {auth }}-\# D B_{\text {obs }}\right) * \frac{0.9 \mathrm{~min}}{D B}
\end{aligned}
$$

\begin{tabular}{|c|c|c|c|c|c|c|c|c|c|}
\hline \multicolumn{2}{|c|}{ Observed feature } & \multicolumn{3}{|c|}{ Authentic Standard } & \multicolumn{4}{|c|}{ Calculation } & \multirow[b]{2}{*}{$\begin{array}{l}\text { Proposed Functional Group } \\
\text { annotation }\end{array}$} \\
\hline Structural annotation & $\mathrm{RT}_{\text {obs }}$ & $\begin{array}{l}\text { Structural } \\
\text { annotation }\end{array}$ & Standard & $\mathrm{RT}_{\text {auth }}$ & $\mathrm{dC}$ & $\mathrm{dDB}$ & $\mathrm{RT}_{\text {calc }}$ & $\mathrm{RT}_{\text {obs }}-\mathrm{RT}_{\text {calc }}$ & \\
\hline FA $10: 3$ none RT-1.9 & 1.9 & FFA 8:0 & Octanoic acid & 2.65 & 2 & 3 & 2.05 & -0.15 & decatrienoic acid (3b) \\
\hline FA $10: 1$ none RT-2.3 & 2.3 & FFA 8:0 & Octanoic acid & 2.65 & 2 & 1 & 3.85 & -1.55 & \\
\hline FA $10: 0$ none RT- 4.3 & 4.3 & FFA 8:0 & Octanoic acid & 2.65 & 2 & 0 & 4.75 & -0.45 & decanoic acid (3b) \\
\hline
\end{tabular}


Table 13. A comparison of theoretical retention times and observed retention times for C20:5 structural isomers.. The theoretical retention time $\left(\mathrm{RT}_{\log \mathrm{P}}\right)$ of the proposed position level annotations of the $\mathrm{C} 20: 5$ one and two oxygen oxylipins were calculated from the equation derived from regressing the RT and the octanol water partition coefficient $(\log \mathrm{P})$ of the C20:4 oxylipin standards (Table 4A). Theoretical RT were compared to observed retention times of structural isomers in the DYEatom dissolved lipidome and the percent error from the observed values were reported $(*$ verified with an authentic standard)

\begin{tabular}{|c|c|c|c|c|c|c|}
\hline \multirow{2}{*}{$\begin{array}{l}\text { Non-volatile } \\
\text { oxylipin } \\
\text { classes }\end{array}$} & \multirow{2}{*}{$\begin{array}{l}\text { Proposed Position } \\
\text { Level Annotation }\end{array}$} & \multirow[b]{2}{*}{$\log \mathrm{P}$} & \multirow[b]{2}{*}{$\mathrm{RT}_{\log \mathrm{P}}$} & \multirow{2}{*}{$\begin{array}{l}\Delta \mathrm{RT}_{\text {vs }} \mathrm{RT}_{(\mathrm{obs}) 1} \\
\text { diHEPE RT- } 2.1\end{array}$} & \multirow{2}{*}{$\begin{array}{l}\Delta \mathrm{RT}_{\text {vs }} \mathrm{RT}_{(\mathrm{obs}) 2} \\
\text { diHEPE RT- } 2.9 \\
\end{array}$} & \multirow[t]{2}{*}{$\Delta R T$ vs $R_{\text {(obs) } 3}$} \\
\hline & & & & & & \\
\hline \multirow{9}{*}{$\begin{array}{c}\text { Dihydroxy } \\
\text { acids } \\
\text { (NVO C20:5 } \\
2 \mathrm{O})\end{array}$} & 4,5 di-HEPE & 3.1 & 2.5 & -0.5 & 0.4 & \\
\hline & 7,8 di-HEPE & 3.6 & 3.1 & -1.0 & -0.2 & \\
\hline & 10,11 di-HEPE & 3.6 & 3.1 & -1.0 & -0.2 & \\
\hline & 13,14 di-HEPE & 3.6 & 3.1 & -1.0 & -0.2 & \\
\hline & 16,17 di-HEPE & 3.3 & 2.8 & -0.7 & 0.1 & \\
\hline & 9,15 di-HEPE & 3.6 & 3.1 & -1 & -0.2 & \\
\hline & 5,11 di-HEPE & 3.3 & 2.8 & -0.7 & 0.1 & \\
\hline & 11,17 di-HEPE & 3.6 & 3.1 & -1 & -0.2 & \\
\hline & & $\log \mathrm{P}$ & $\mathrm{RT}_{\log \mathrm{P}}$ & HpEPE RT-4.9 & NVO C20:5 2O RT-6.7 & NVO C20:5 207.8 \\
\hline \multirow{5}{*}{$\begin{array}{c}\text { Hydroperoxy } \\
\text { acids } \\
\text { (NVO C20:5 } \\
2 \mathrm{O})\end{array}$} & $5 \mathrm{HpEPE}$ & 4.5 & 4.5 & 0.4 & 2.2 & 3.3 \\
\hline & 9HрЕРЕ & 4.8 & 4.8 & 0.1 & 1.9 & 3.0 \\
\hline & $11 \mathrm{HpEPE}$ & 4.8 & 4.8 & 0.1 & 1.9 & 3.0 \\
\hline & $14 \mathrm{HpEPE}$ & 4.8 & 4.8 & 0.1 & 1.9 & 3.0 \\
\hline & $15 \mathrm{HpEPE}$ & 4.8 & 4.8 & 0.1 & 1.9 & 3.0 \\
\hline \multirow{4}{*}{$\begin{array}{c}\text { Hydroxy epoxy } \\
\text { acids } \\
\text { (NVO C20:5 } \\
\text { 2O) } \\
\end{array}$} & & $\log \mathrm{P}$ & $\mathrm{RT}_{\log \mathrm{P}}$ & HpEPE RT-4.9 & NVO C20:5 2O RT-6.7 & NVO C20:5 207.8 \\
\hline & $15(13,14)$ HepETE & 4.6 & 4.6 & 0.3 & 2.1 & 3.2 \\
\hline & $8(9,10)$ HepETE & 4.6 & 4.6 & 0.3 & 2.1 & 3.2 \\
\hline & & $\log \mathrm{P}$ & $\mathrm{RT}_{\log \mathrm{P}}$ & HEPE RT-4.6* & NVO C20:5 1O RT-5.9 & NVO C20:5 1O RT-6.8 \\
\hline \multirow{7}{*}{$\begin{array}{l}\text { Hydroxy } \\
\text { acids (NVO } \\
\text { C20:5 1O) }\end{array}$} & 5HEPE & 4.3 & 4.1 & 0.5 & 1.8 & 2.7 \\
\hline & 9- HEPE & 4.5 & 4.4 & 0.1 & 1.5 & 2.4 \\
\hline & 11-HEPE & 4.5 & 4.4 & 0.1 & 1.5 & 2.4 \\
\hline & 12-HEPE & 4.5 & 4.4 & 0.1 & 1.5 & 2.4 \\
\hline & 14-HEPE & 4.5 & 4.4 & 0.1 & 1.5 & 2.4 \\
\hline & 15-HEPE & 4.5 & 4.4 & 0.1 & 1.5 & 2.4 \\
\hline & & $\log \mathrm{P}$ & $\mathrm{RT}_{\log \mathrm{P}}$ & HEPE RT-4.6* & NVO C20:5 1O RT-5.9 & NVO C20:5 10 RT-6.8 \\
\hline \multirow{5}{*}{$\begin{array}{c}\text { Oxo acids } \\
\text { (NVO C20:5 } \\
10)\end{array}$} & 17-oxo ETE & 4.6 & 4.6 & 0 & 1.3 & 2.2 \\
\hline & 15-oxo ETE & 5.1 & 5.3 & -0.7 & 0.6 & 1.5 \\
\hline & 12 oxo ETE & 5.3 & 5.6 & -1.0 & 0.3 & 1.2 \\
\hline & 8 oxo ETE & 4.6 & 4.5 & 0.1 & 1.4 & 2.3 \\
\hline & 5 oxo ETE & 4.3 & 4.2 & 0.4 & 1.7 & 2.4 \\
\hline
\end{tabular}


Table 14A. Covariance PCA results for the DYEatom cruise dissolved lipidomes (active cases).

\begin{tabular}{lcccccc}
\hline & PC 1 (83.44\%) & $\begin{array}{c}\text { PC2 } \\
(9.38 \%)\end{array}$ & $\begin{array}{c}\text { Contribution } \\
\text { PC1 }\end{array}$ & $\begin{array}{c}\text { Contribution } \\
\text { PC2 }\end{array}$ & $\begin{array}{c}\cos ^{2} \\
\text { PC1 }\end{array}$ & $\begin{array}{c}\cos ^{2} \\
\text { PC2 }\end{array}$ \\
\hline St1-10m & $-6.19 \mathrm{E}+07$ & $-1.17 \mathrm{E}+08$ & 0.34 & 10.81 & 0.12 & 0.45 \\
St4-2m & $1.56 \mathrm{E}+08$ & $-1.68 \mathrm{E}+07$ & 2.16 & 0.22 & 0.89 & 0.01 \\
St4-9m & $8.90 \mathrm{E}+07$ & $-1.26 \mathrm{E}+08$ & 0.70 & 12.54 & 0.18 & 0.36 \\
St6-4m & $2.36 \mathrm{E}+08$ & $8.18 \mathrm{E}+07$ & 4.93 & 5.25 & 0.88 & 0.11 \\
St6-35m & $2.41 \mathrm{E}+08$ & $8.27 \mathrm{E}+07$ & 5.12 & 5.37 & 0.88 & 0.10 \\
St7-8 & $-3.26 \mathrm{E}+08$ & $-1.54 \mathrm{E}+08$ & 9.40 & 18.50 & 0.64 & 0.14 \\
St8-1m & $5.09 \mathrm{E}+07$ & $9.33 \mathrm{E}+07$ & 0.23 & 6.83 & 0.20 & 0.67 \\
St8-4m & $2.17 \mathrm{E}+08$ & $7.94 \mathrm{E}+07$ & 4.16 & 4.95 & 0.87 & 0.12 \\
St9-7m & $2.34 \mathrm{E}+08$ & $8.37 \mathrm{E}+07$ & 4.85 & 5.49 & 0.87 & 0.11 \\
St10-10m & $-8.78 \mathrm{E}+08$ & $1.35 \mathrm{E}+08$ & 67.98 & 14.30 & 0.97 & 0.02 \\
St11-2m & $4.11 \mathrm{E}+07$ & $-1.42 \mathrm{E}+08$ & 0.15 & 15.75 & 0.05 & 0.58
\end{tabular}


Table 14B. Covariance PCA results for the 19 significantly varying molecular species (active variables).

\begin{tabular}{|c|c|c|c|c|c|c|}
\hline & $\begin{array}{c}\text { PC 1 } \\
(83.44 \%)\end{array}$ & $\begin{array}{c}\text { PC2 } \\
(9.38 \%)\end{array}$ & $\begin{array}{l}\text { Contribution } \\
\text { PC1 }\end{array}$ & $\begin{array}{l}\text { Contribution } \\
\text { PC2 }\end{array}$ & $\begin{array}{l}\cos ^{2} \\
\mathrm{PC} 1\end{array}$ & $\begin{array}{l}\cos ^{2} \\
\mathrm{PC} 2\end{array}$ \\
\hline EPA RT-7.7 & $-2.48 \mathrm{E}+08$ & $-3.90 \mathrm{E}+07$ & 0.54 & 0.12 & 0 & 0 \\
\hline diHEPE RT-2.1 & $-1.14 \mathrm{E}+08$ & $5.20 \mathrm{E}+07$ & 0.12 & 0.21 & 1 & 1 \\
\hline HEPE RT-4.6 & $-7.92 \mathrm{E}+07$ & $1.97 \mathrm{E}+07$ & 0.06 & 0.03 & 1 & 1 \\
\hline NVO 20:4 3O RT-1.7 & $-7.58 \mathrm{E}+07$ & $3.58 \mathrm{E}+07$ & 0.05 & 0.10 & 0 & 1 \\
\hline HpEPE RT-4.9 & $-7.22 \mathrm{E}+07$ & $-2.98 \mathrm{E}+06$ & 0.05 & 0.00 & 1 & 0 \\
\hline NVO 16:2 1O RT-4.6 & $-6.49 \mathrm{E}+07$ & $1.36 \mathrm{E}+07$ & 0.04 & 0.01 & 1 & 1 \\
\hline FFA 16:4 none RT-5.7 & $-6.46 \mathrm{E}+07$ & $-7.00 \mathrm{E}+07$ & 0.04 & 0.39 & 0 & 0 \\
\hline NVO 18:2 2O RT-3.2 & $-5.83 \mathrm{E}+07$ & $2.42 \mathrm{E}+07$ & 0.03 & 0.05 & & 1 \\
\hline HpHME RT-4.6 & $-5.16 \mathrm{E}+07$ & $1.06 \mathrm{E}+07$ & 0.02 & 0.01 & 1 & 1 \\
\hline NVO 16:1 1O RT-4.3 & $-4.70 \mathrm{E}+07$ & $2.30 \mathrm{E}+07$ & 0.02 & 0.04 & 1 & 1 \\
\hline KHDE RT-4 & $-2.87 \mathrm{E}+07$ & $1.14 \mathrm{E}+07$ & 0.01 & 0.01 & 1 & 1 \\
\hline diHEPE RT-2.9 & $-2.42 \mathrm{E}+07$ & $6.27 \mathrm{E}+06$ & 0.01 & 0.00 & 1 & 1 \\
\hline FFA 16:2 RT-7.5 & $-2.23 \mathrm{E}+07$ & $-9.40 \mathrm{E}+05$ & 0.00 & 0.00 & 1 & 0 \\
\hline FFA 16:3 RT-6.8 & $-2.13 \mathrm{E}+07$ & $-7.18 \mathrm{E}+06$ & 0.00 & 0.00 & 1 & 0 \\
\hline FFA 18:4 RT-7.3 & $-1.94 \mathrm{E}+07$ & $-7.79 E+06$ & 0.00 & 0.00 & 1 & 1 \\
\hline DHA RT-8.2 & $-1.94 \mathrm{E}+07$ & $-1.23 \mathrm{E}+06$ & 0.00 & 0.00 & 1 & 1 \\
\hline NVO 18:1 1O RT-7.3 & $-1.93 E+07$ & $7.17 \mathrm{E}+06$ & 0.00 & 0.00 & 1 & 1 \\
\hline $\begin{array}{l}\text { Decatrienoic acid RT-1.9 } \\
\text { (3b) }\end{array}$ & $-1.92 \mathrm{E}+07$ & $7.31 \mathrm{E}+06$ & 0.00 & 0.00 & 1 & 1 \\
\hline FFA 18:5 RT-6.7 & $-1.51 \mathrm{E}+07$ & $-8.18 \mathrm{E}+06$ & 0.00 & 0.01 & 1 & 1 \\
\hline
\end{tabular}

Table 14C. Covariance PCA results for the core supplemental variables.

\begin{tabular}{lcccc}
\hline & $\begin{array}{c}\text { PC } 1 \\
(83.44 \%)\end{array}$ & $\begin{array}{c}\text { PC2 } \\
(9.38 \%)\end{array}$ & $\cos ^{2} \mathrm{PC} 1$ & $\cos ^{2} \mathrm{PC} 2$ \\
\hline Total Chl & 4080 & 285 & $4.12 \mathrm{E}+05$ & $2.48 \mathrm{E}+06$ \\
Chl a & 10982 & -774 & $3.56 \mathrm{E}+06$ & $4.14 \mathrm{E}+05$ \\
Phaeo & -13174 & 1990 & $6.02 \mathrm{E}+06$ & $3.58 \mathrm{E}+06$ \\
bSi & -12477 & 2265 & $2.95 \mathrm{E}+06$ & $3.01 \mathrm{E}+06$ \\
dSi & 9586 & -630 & $2.99 \mathrm{E}+06$ & $6.16 \mathrm{E}+06$ \\
Nitrogen & 6768 & -330 & $2.47 \mathrm{E}+06$ & $4.72 \mathrm{E}+05$ \\
SRP & 144 & -4 & $4.72 \mathrm{E}+05$ & $7.35 \mathrm{E}-01$ \\
N:P & 7788 & 1028 & $4.62 \mathrm{E}+05$ & $1.00 \mathrm{E}+00$
\end{tabular}


Table 15. Regression of supplemental variables against PC1 and PC2.

\begin{tabular}{|c|c|c|c|c|c|c|c|c|}
\hline \multirow[b]{2}{*}{ Supplemental Variable } & \multicolumn{4}{|c|}{ PC1 } & \multicolumn{4}{|c|}{$\mathrm{PC} 2$} \\
\hline & Slope & Intercept & $\mathrm{R}^{\wedge} 2$ & $\begin{array}{c}\text { Significance } \\
\text { F }\end{array}$ & Slope & Intercept & $\mathrm{R}^{\wedge} 2$ & $\begin{array}{c}\text { Significance } \\
\text { F }\end{array}$ \\
\hline Total Chl & $-4.77 E-09$ & 10.58 & 0.074 & 0.448 & $-1.31 \mathrm{E}-08$ & 10.58 & 0.062 & 0.487 \\
\hline Chl a & $-1.53 \mathrm{E}-10$ & 8.07 & 0.000 & 0.980 & $-2.44 \mathrm{E}-08$ & 7.87 & 0.240 & 0.151 \\
\hline Phaeophytin & $-8.96 \mathrm{E}-09$ & 4.87 & 0.317 & 0.090 & 2.19E-08 & 5.26 & 0.213 & 0.180 \\
\hline bSi & $-1.25 \mathrm{E}-08$ & 8.91 & 0.368 & 0.063 & $2.35 \mathrm{E}-08$ & 9.41 & 0.146 & 0.275 \\
\hline $\mathrm{dSi}$ & $-3.71 \mathrm{E}-10$ & 4.07 & 0.001 & 0.944 & $-1.14 \mathrm{E}-08$ & 3.98 & 0.071 & 0.457 \\
\hline $\mathrm{NO} 3+\mathrm{NO} 2$ & $-1.25 \mathrm{E}-09$ & 2.06 & 0.016 & 0.725 & $-8.63 E-09$ & 2.02 & 0.088 & 0.406 \\
\hline SRP & $-6.91 E-11$ & 0.17 & 0.050 & 0.533 & $3.78 \mathrm{E}-10$ & 0.18 & 0.169 & 0.238 \\
\hline $\mathbf{N}: \mathbf{P}$ & $-6.77 \mathrm{E}-09$ & 9.72 & 0.038 & 0.590 & $-6.08 \mathrm{E}-08$ & 9.38 & 0.344 & 0.075 \\
\hline Si-stress & $1.25 \mathrm{E}-10$ & 0.54 & 0.016 & 0.745 & $-6.18 \mathrm{E}-10$ & 0.54 & 0.037 & 0.620 \\
\hline Leucinase & $-1.29 \mathrm{E}-11$ & 0.21 & 0.001 & 0.928 & $-9.85 E-11$ & 0.22 & 0.007 & 0.847 \\
\hline Serinase & $-6.77 E-11$ & 0.08 & 0.339 & 0.130 & $3.27 \mathrm{E}-11$ & 0.08 & 0.006 & 0.854 \\
\hline Glycinase & $-2.44 \mathrm{E}-11$ & 0.11 & 0.028 & 0.691 & $-4.38 \mathrm{E}-11$ & 0.11 & 0.007 & 0.843 \\
\hline APase & $-3.54 \mathrm{E}-12$ & 0.01 & 0.133 & 0.374 & $1.20 \mathrm{E}-12$ & 0.01 & 0.001 & 0.935 \\
\hline Grazing Rate & $5.83 \mathrm{E}-09$ & -0.39 & 0.075 & 0.824 & $6.71 \mathrm{E}-09$ & 0.46 & 0.190 & 0.713 \\
\hline Growth Rate & $1.46 \mathrm{E}-09$ & 0.49 & 0.005 & 0.955 & 3.60E-09 & 0.61 & 0.059 & 0.843 \\
\hline Total RNA Viral Reads & $-6.79 \mathrm{E}-12$ & 0.00 & 0.781 & 0.008 & $1.28 \mathrm{E}-11$ & 0.00 & 0.153 & 0.386 \\
\hline $\begin{array}{r}\% \text { Phytophthora } \\
\text { infestans RNA virus } 1\end{array}$ & $-3.44 \mathrm{E}-08$ & 54.02 & 0.121 & 0.121 & $3.14 \mathrm{E}-07$ & 39.61 & 0.560 & 0.053 \\
\hline \% Marine RNA virus & & & & & & & & \\
\hline JP-A & $3.55 \mathrm{E}-09$ & 11.24 & 0.003 & 0.902 & $-2.43 \mathrm{E}-07$ & 22.18 & 0.871 & 0.002 \\
\hline \% Chaetoceros sp. RNA & & & & & & & & \\
\hline virus 2 & 2.24E-09 & 3.89 & 0.019 & 0.767 & $-6.48 \mathrm{E}-08$ & 6.83 & 0.892 & 0.001 \\
\hline
\end{tabular}




\begin{tabular}{|c|c|c|c|c|c|c|}
\hline Group & & Molecular Species & & $\begin{array}{c}\text { Avg \# } \\
\text { C }\end{array}$ & $\begin{array}{c}\text { Avg \# } \\
\text { DB }\end{array}$ & $\begin{array}{c}\text { Avg \# } \\
\text { O }\end{array}$ \\
\hline $1(\mathrm{~N}=5)$ & $\begin{array}{l}\text { NVO 18:1 } 30 \text { RT-5.9 } \\
\text { NVO 22:2 } 4 \text { O RT-2.3 }\end{array}$ & $\begin{array}{l}\text { NVO 20:1 3O RT-5.6 } \\
\text { NVO 20:1 3O RT-7.3 }\end{array}$ & NVO 20:2 4O RT-3.4 & 20.0 & 1.4 & 3.4 \\
\hline $2(\mathrm{~N}=14)$ & $\begin{array}{l}\text { NVO 20:3 } 3 \text { O RT-7.1 } \\
\text { NVO 16:1 } 3 \text { O RT-3.9 } \\
\text { NVO 20:3 } 2 \text { O RT-4.5 } \\
\text { NVO 20:2 } 3 \text { O RT-5.4 } \\
\text { FFA 16:2 RT-4.1 }\end{array}$ & $\begin{array}{l}\text { FFA } 18: 5 \text { RT-3.5 } \\
\text { NVO 20:3 2O RT-6.8 } \\
\mathrm{C}_{10} \mathrm{H}_{12} \mathrm{O} \text { RT-4.9 } \\
\text { NVO 20:4 4O RT-4.6 } \\
\text { NVO 22:5 1O RT-6.4 }\end{array}$ & $\begin{array}{l}\text { NVO 14:0 } 20 \text { RT-2.2 } \\
\text { NVO 22:4 } 3 \text { O RT-3 } \\
\text { NVO 16:1 } 3 \text { O RT-1.8 } \\
\text { NVO 20:4 3O RT-4.1 }\end{array}$ & 18.1 & 2.8 & 2.1 \\
\hline $3(\mathrm{~N}=2)$ & NVO 10:4 4 O RT-2.7 & NVO 22:0 4O RT-2.5 & & 16.0 & 2.0 & 4.0 \\
\hline $4(\mathrm{~N}=2)$ & NVO 14:1 4O RT-1.5 & NVO 20:2 4O RT-4.4 & & 17.0 & 1.5 & 4.0 \\
\hline $5(\mathrm{~N}=1)$ & FFA 16:2 RT-5.2 & & & 16.0 & 2.0 & 0.0 \\
\hline $6(\mathrm{~N}=7)$ & $\begin{array}{l}\text { NVO } 22: 5 \text { 2O RT-2.9 } \\
\mathrm{C}_{12} \mathrm{H}_{14} \mathrm{O} \text { RT }-4.9 \\
\mathrm{C}_{10} \mathrm{H}_{12} \mathrm{O} \text { RT-3.2 }\end{array}$ & $\begin{array}{l}\text { NVO 22:0 3O RT-9.5 } \\
\text { NVO 22:2 3O RT-7.3 } \\
\text { NVO 20:5 3O RT-6.6 }\end{array}$ & FFA 22:3 RT-4.9 & 18.6 & 3.4 & 1.6 \\
\hline $7(\mathrm{~N}=2)$ & FFA 16:3 RT-3.8 & FFA 16:1 RT-2.2 & & 16.0 & 2.0 & 0.0 \\
\hline $8(\mathrm{~N}=3)$ & HETrE RT-5.5 & NVO 16:2 3O RT-1.8 & NVO 22:4 4O RT-2.5 & 19.3 & 3.0 & 2.7 \\
\hline $9(\mathrm{~N}=1)$ & NVO 18:0 3O RT-2.2 & & & 18.0 & 0.0 & 3.0 \\
\hline $10(\mathrm{~N}=1)$ & FFA 18:0 RT-3.2 & & & 18.0 & 0.0 & 0.0 \\
\hline $11(\mathrm{~N}=5)$ & $\begin{array}{l}\text { NVO 14:0 1O RT-4.8 } \\
\text { HETE RT-5.5 }\end{array}$ & $\begin{array}{l}\mathrm{C}_{10} \mathrm{H}_{16} \mathrm{O} \text { RT-1.5 } \\
\text { NVO 12:4 4O RT-1.3 }\end{array}$ & FFA 16:4 RT-3 & 14.4 & 2.8 & 1.2 \\
\hline $12(\mathrm{~N}=8)$ & 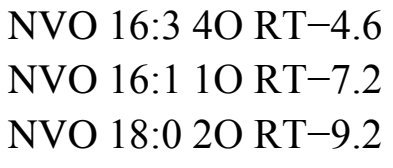 & $\begin{array}{l}\text { NVO 18:0 1O RT-7.3 } \\
\text { Decanoic acid RT-4.3 } \\
\text { NVO 10:4 1O RT-2 }\end{array}$ & $\begin{array}{l}\text { FFA 18:2 RT-8.5 } \\
\text { NVO 20:4 2O RT-2.3 }\end{array}$ & 15.8 & 1.8 & 1.4 \\
\hline
\end{tabular}


Table 16. (continued) Groups of molecular species with significantly similar abundances across the cruise track

\begin{tabular}{|c|c|c|c|c|c|c|}
\hline Group & \multicolumn{3}{|c|}{ Molecular Species } & $\begin{array}{c}\text { Avg \# } \\
\text { C }\end{array}$ & $\begin{array}{c}\text { Avg \# } \\
\text { DB }\end{array}$ & $\begin{array}{c}\text { Avg \# } \\
\text { O }\end{array}$ \\
\hline $\begin{array}{c}13 \\
(\mathrm{~N}=25)\end{array}$ & $\begin{array}{l}\text { NVO 14:2 } 2 \text { O RT-1.9 } \\
\text { NVO 16:0 } 10 \text { RT-5.2 } \\
\text { NVO 16:4 } 3 \text { O RT-1.5 } \\
\text { Decatrienoic acid RT-1.9 } \\
\text { NVO 12:4 } 2 \text { O RT-1.5 } \\
\text { NVO 12:2 } 30 \text { RT-1.5 } \\
\text { NVO 18:5 3O RT-1.5 } \\
\text { NVO 18:4 } 4 \text { O RT-1.5 } \\
\text { NVO 14:2 } 30 \text { RT-1.5 }\end{array}$ & $\begin{array}{l}\text { FFA 16:4 RT-4.2 } \\
\text { NVO 10:2 } 4 \text { O RT-2.9 } \\
\text { NVO 16:0 } 2 \text { O RT-2.1 } \\
\text { NVO 18:5 } 2 \text { O RT-1.6 } \\
\text { NVO 14:2 } 4 \text { O RT-1.5 } \\
\text { NVO 16:2 } 4 \text { O RT-1.7 } \\
\text { NVO 18:4 } 2 \text { O RT-2 } \\
\text { NVO 14:2 } 10 \text { RT-4 } \\
\text { NVO } 12: 3 \text { 3O RT-1.4 }\end{array}$ & $\begin{array}{l}\text { NVO 22:3 } 2 \text { O RT-10.2 } \\
\text { NVO 14:1 } 10 \text { RT-2.6 } \\
\text { NVO 12:0 } 10 \text { RT-2.7 } \\
\text { NVO 18:1 } 10 \text { RT-6.4 } \\
\text { FFA 12:0 RT-10 } \\
\text { NVO 10:3 1O RT-1.6 } \\
\text { FFA 20:0 RT-11.6 }\end{array}$ & 14.9 & 2.3 & 2.0 \\
\hline $14(\mathrm{~N}=1)$ & NVO 12:3 2O RT-1.6 & & & 12.0 & 3.0 & 2.0 \\
\hline $15(\mathrm{~N}=3)$ & NVO $12: 43 \mathrm{O}$ RT-1.3 & NVO $16: 4$ 4O RT-1.4 & NVO 18:5 4O RT-1.4 & 15.3 & 4.3 & 3.7 \\
\hline $\begin{array}{c}16 \\
(\mathrm{~N}=30)\end{array}$ & $\begin{array}{l}\text { NVO 12:4 1O RT-1.7 } \\
\text { NVO 20:5 4O RT-1.7 } \\
\text { NVO 18:3 3O RT-1.8 } \\
\text { FFA 12:4 RT-1.4 } \\
\text { NVO 18:3 4O RT-1.6 } \\
\text { FFA 14:1 RT-7.2 } \\
\text { FFA 18:1 RT-9.3 } \\
\text { NVO 22:4 4O RT-1.7 } \\
\text { NVO 12:2 4O RT-1.4 } \\
\text { NVO 22:5 4O RT-1.6 }\end{array}$ & $\begin{array}{l}\text { NVO 16:4 } 2 \text { O RT-1.7 } \\
\text { NVO 12:3 } 1 \text { O RT-1.8 } \\
\text { NVO 16:3 } 3 \text { O RT-1.7 } \\
\text { NVO 18:2 } 4 \text { O RT-1.7 } \\
\text { NVO 20:3 } 4 \text { O RT-1.7 } \\
\text { NVO 16:3 } 2 \text { O RT-2.5 } \\
\text { NVO 12:2 } 10 \text { RT-1.7 } \\
\text { NVO 20:5 } 3 \text { O RT-1.7 } \\
\text { NVO 22:5 } 3 \text { O RT-2 } \\
\text { NVO 14:1 } 10 \text { RT-3.6 }\end{array}$ & $\begin{array}{l}\text { NVO 22:6 3O RT-1.9 } \\
\text { FFA 10:1 RT-2.3 } \\
\text { NVO 12:1 1O RT-1.8 } \\
\text { NVO 12:0 1O RT-2 } \\
\text { NVO 14:2 1O RT-2.3 } \\
\text { FFA 16:3 RT-5.6 } \\
\text { NVO 16:3 2O RT-1.8 } \\
\text { NVO 16:3 4O RT-1.6 } \\
\text { NVO 10:0 1O RT-1.8 } \\
\text { NVO 12:0 2O RT-4.1 }\end{array}$ & 15.8 & 2.7 & 2.1 \\
\hline $17(\mathrm{~N}=2)$ & FFA 18:0 RT-7.6 & NVO 18:1 $1 O R T-7.3$ & & 18.0 & 0.5 & 0.5 \\
\hline $18(\mathrm{~N}=4)$ & $\begin{array}{l}\text { NVO 20:3 } 30 \text { RT-1.8 } \\
\text { NVO 22:6 } 10 \text { RT-5.7 }\end{array}$ & NVO 16:0 10 RT-7 & diHETE RT-3.7 & 19.5 & 3.3 & 1.8 \\
\hline
\end{tabular}


Table 16. (continued) Groups of molecular species with significantly similar abundances across the cruise track

\begin{tabular}{|c|c|c|c|c|c|c|}
\hline Group & & Molecular Species & & $\begin{array}{c}\operatorname{Avg} \# \\
\mathrm{C}\end{array}$ & $\begin{array}{c}\text { Avg \# } \\
\text { DB }\end{array}$ & $\begin{array}{c}\text { Avg \# } \\
\mathrm{O}\end{array}$ \\
\hline $\begin{array}{c}19 \\
(\mathrm{~N}=10)\end{array}$ & $\begin{array}{l}\text { NVO 18:2 1O RT-5.5 } \\
\text { NVO 20:4 1O RT-6.7 } \\
\text { NVO 18:2 1O RT-6.7 } \\
\text { HETrE RT-7.3 }\end{array}$ & $\begin{array}{l}\text { NVO 18:3 } 2 \text { O RT-1.8 } \\
\text { NVO 18:1 2O RT-6.7 } \\
\text { NVO 12:1 } 10 \text { RT-2.7 } \\
\text { FFA 12:2 RT-3.1 }\end{array}$ & $\begin{array}{l}\text { NVO 14:1 } 10 \text { RT-4.5 } \\
\text { NVO 14:1 } 2 \text { O RT-2.8 }\end{array}$ & 16.4 & 2.0 & 1.2 \\
\hline $20(\mathrm{~N}=2)$ & FFA 16:4 RT-5.7 & FFA 16:3 RT-6.8 & & 16.0 & 3.5 & 0.0 \\
\hline $21(\mathrm{~N}=1)$ & NVO 22:5 4O RT-2.4 & & & 22.0 & 5.0 & 4.0 \\
\hline $22(\mathrm{~N}=2)$ & FFA 18:3 RT-7.9 & DHA RT-8.2 & & 20.0 & 4.5 & 0.0 \\
\hline $23(\mathrm{~N}=2)$ & FFA 18:5 RT-6.7 & FFA 18:4 RT-7.3 & & 18.0 & 4.5 & 0.0 \\
\hline $24(\mathrm{~N}=5)$ & $\begin{array}{l}\text { KHTrE/HHTE RT-2.1 } \\
\text { HpHTE RT-2.3 }\end{array}$ & $\begin{array}{l}\text { NVO 16:4 1O RT-2.7 } \\
\text { FFA 16:2 RT-7.5 }\end{array}$ & EPA RT-7.7 & 16.8 & 3.8 & 0.8 \\
\hline $25(\mathrm{~N}=1)$ & NVO 18:2 $2 O R T-3.2$ & & & 18.0 & 2.0 & 2.0 \\
\hline $26(\mathrm{~N}=2)$ & HEPE RT-4.6 & diHEPE RT-2.9 & & 20.0 & 5.0 & 1.5 \\
\hline $27(\mathrm{~N}=2)$ & KHDE RT-4 & NVO 20:4 30 RT-1.7 & & 18.0 & 3.0 & 2.0 \\
\hline $28(\mathrm{~N}=3)$ & HHME RT-4.3 & NVO 16:2 1O RT-4.6 & diHEPE RT-2.1 & 17.3 & 2.7 & 1.3 \\
\hline $29(\mathrm{~N}=4)$ & $\begin{array}{l}\mathrm{C}_{10} \mathrm{H}_{12} \mathrm{O} \text { RT }-1.7 \\
\mathrm{NVO} 16: 02 \mathrm{OTT}-8\end{array}$ & FFA $10: 2$ RT -1.5 & NVO 12:1 4O RT-1.5 & 12.0 & 1.8 & 1.5 \\
\hline $30(\mathrm{~N}=4)$ & $\begin{array}{l}\text { NVO 10:3 1O RT-4.9 } \\
\text { FFA 12:3 RT-3 }\end{array}$ & $\underline{\text { HpHTrE RT-3.9 }}$ & NVO 18:0 2O RT-4.4 & 14.0 & 2.3 & 1.3 \\
\hline
\end{tabular}


Table 16. (continued) Groups of molecular species with significantly similar abundances across the cruise track

\begin{tabular}{|c|c|c|c|c|c|c|}
\hline Group & & Molecular Species & & $\underset{\mathrm{C}}{\operatorname{Avg} \#}$ & $\begin{array}{c}\text { Avg \# } \\
\text { DB }\end{array}$ & $\begin{array}{c}\text { Avg \# } \\
\mathrm{O}\end{array}$ \\
\hline $\begin{array}{c}31 \\
(N=46)\end{array}$ & $\begin{array}{l}\text { NVO 18:1 2O RT-2.4 } \\
\text { FFA 24:0 RT-15.2 } \\
\text { FFA 26:0 RT-17.4 } \\
\text { NVO 22:5 4O RT-3.1 } \\
\text { KHTrE RT-4.6 } \\
\text { NVO 22:6 3O RT-4.6 } \\
\text { NVO 22:6 2O RT-4 } \\
\text { NVO 18:2 2O RT-7.5 } \\
\text { NVO 20:4 2O RT-8.2 } \\
\text { KHDE RT-2.4 } \\
\text { NVO 18:5 1O RT-3.5 } \\
\text { NVO 18:4 2O RT-3.7 } \\
\text { KHDE RT-3.4 } \\
\text { NVO 18:4 1O RT-3.3 } \\
\text { FFA 20:3 RT-8.8 } \\
\text { NVO 18:3 2O RT-3.5 }\end{array}$ & $\begin{array}{l}\text { NVO 18:1 } 2 \text { R RT-8.2 } \\
\text { NVO 20:5 } 3 \text { O RT-3.2 } \\
\text { NVO 22:5 } 20 \text { RT-7.9 } \\
\text { NVO 20:5 } 10 \text { RT-5.9 } \\
\text { HpHME RT-4.6 } \\
\text { HpEPE RT-4.9 } \\
\text { NVO 10:4 2O RT-1.6 } \\
\text { NVO 14:1 2O RT-3.8 } \\
\text { NVO 18:3 2O RT-2.5 } \\
\text { NVO 22:6 2O RT-7.2 } \\
\text { NVO 20:1 2O RT-9.3 } \\
\text { NVO 22:0 } 40 \text { RT-9.6 } \\
\text { NVO 10:4 } 40 \text { RT-1.5 } \\
\text { NVO 14:0 } 10 \text { RT-2.8 } \\
\text { HpHDE RT-3.4 } \\
\text { NVO 16:2 2O RT-2 }\end{array}$ & $\begin{array}{l}\text { NVO 16:1 2O RT-2.5 } \\
\text { NVO 18:3 1O RT-5.7 } \\
\text { NVO 18:3 3O RT-2.8 } \\
\text { NVO 22:6 } 10 \text { RT-5.2 } \\
\text { NVO 18:3 4O RT-6.8 } \\
\text { FFA 22:0 RT-13.2 } \\
\text { FFA 14:2 RT-3.8 } \\
\text { NVO 22:4 4O RT-3 } \\
\text { HHDE/KHME RT-3.1 } \\
\text { NVO 18:5 2O RT-2.9 } \\
\text { NVO 18:1 2O RT-3.1 } \\
\text { NVO 18:1 2O RT-3.8 } \\
\text { NVO 18:2 4O RT-2.4 } \\
\text { NVO 20:3 2O RT-9 }\end{array}$ & 18.5 & 2.9 & 1.9 \\
\hline $32(\mathrm{~N}=5)$ & $\begin{array}{l}\text { NVO 10:2 } 10 \text { RT-2.2 } \\
\text { NVO 20:0 2O RT-10.4 }\end{array}$ & $\begin{array}{l}\text { NVO 18:1 } 30 \text { RT-1.9 } \\
\text { NVO 16:2 } 10 \text { RT-7 }\end{array}$ & NVO 16:1 2O RT-5.9 & 16.0 & 1.2 & 1.8 \\
\hline $33(\mathrm{~N}=3)$ & NVO 20:2 10 RT-5.4 & NVO 14:1 3O RT-1.6 & NVO 20:5 10 RT-7.1 & 18.0 & 2.7 & 1.7 \\
\hline $34(\mathrm{~N}=3)$ & NVO 22:5 3O RT-7.7 & NVO 20:2 2O RT-8.5 & NVO 20:5 4O RT-3.2 & 20.7 & 4.0 & 3.0 \\
\hline $35(\mathrm{~N}=2)$ & NVO 20:3 2O RT-5.8 & NVO 20:2 2O RT-7.3 & & 20.0 & 2.5 & 2.0 \\
\hline $36(\mathrm{~N}=3)$ & NVO 22:6 2O RT-7.9 & FFA $20: 2$ RT-9.5 & NVO 22:5 2O RT-6.7 & 21.3 & 4.3 & 1.3 \\
\hline $37(\mathrm{~N}=3)$ & PUA 12:3 RT-4.9 & FFA 24:1 RT-13.2 & $\mathrm{C}_{10} \mathrm{H}_{14} \mathrm{O} \mathrm{RT}-4.9$ & 15.3 & 2.3 & 0.0 \\
\hline $38(\mathrm{~N}=3)$ & NVO 20:2 4O RT-2.7 & NVO 18:3 2O RT-5.4 & NVO 20:5 2O RT-6.7 & 19.3 & 3.3 & 2.7 \\
\hline
\end{tabular}


Table 16. (continued) Groups of molecular species with significantly similar abundances across the cruise track

\begin{tabular}{|c|c|c|c|c|c|c|}
\hline Group & \multicolumn{3}{|c|}{ Molecular Species } & $\begin{array}{l}\text { Avg } \\
\# \text { C }\end{array}$ & $\begin{array}{c}\text { Avg \# } \\
\text { DB }\end{array}$ & Avg \# O \\
\hline $39(\mathrm{~N}=19)$ & $\begin{array}{l}\text { NVO 14:0 } 20 \text { RT-6.8 } \\
\text { NVO 20:3 2O RT-2.4 } \\
\text { FFA 22:5 RT-8.5 } \\
\text { NVO 20:4 2O RT-7.3 } \\
\text { NVO 22:5 4O RT-4.2 } \\
\text { NVO 22:4 } 4 \text { O RT-3.6 } \\
\text { NVO 22:6 } 10 \text { RT-6.6 }\end{array}$ & $\begin{array}{l}\text { NVO 22:4 } 3 \text { O RT-2.1 } \\
\text { NVO 20:3 2O RT-7.2 } \\
\text { HpODE RT-5.7 } \\
\text { NVO 22:6 2O RT-5.9 } \\
\text { FFA 14:2 RT-6.1 } \\
\text { NVO 18:4 2O RT-5.7 } \\
\text { NVO 18:3 2O RT-6.8 }\end{array}$ & $\begin{array}{l}\text { NVO 18:3 2O RT-7.9 } \\
\text { NVO 20:5 2O RT-7.5 } \\
\text { HpETE RT-5.9 } \\
\text { HpOTrE RT-4.6 } \\
\text { NVO 20:3 3O RT-3.9 }\end{array}$ & 19.5 & 3.6 & 2.1 \\
\hline $40(\mathrm{~N}=24)$ & $\begin{array}{l}\text { NVO 10:3 4O RT-2.6 } \\
\text { NVO 10:0 2O RT-4.7 } \\
\text { NVO 12:2 2O RT-7.1 } \\
\text { HpODE RT-4.5 } \\
\text { NVO 22:4 3O RT-7 } \\
\text { NVO 24:1 2O RT-11.2 } \\
\text { FFA 12:4 RT-4.9 } \\
\text { FFA 12:3 RT-2.2 }\end{array}$ & $\begin{array}{l}\text { NVO 14:2 } 10 \text { RT-4.8 } \\
\text { FFA 20:0 RT-11 } \\
\text { NVO 16:1 2O RT-7.1 } \\
\text { NVO 22:6 } 4 \text { O RT-4.5 } \\
\text { NVO 14:2 2O RT-3.5 } \\
\text { NVO 20:0 } 10 \text { RT-8.3 } \\
\text { NVO 14:1 3O RT-2.2 } \\
\text { HETrE RT-6.5 }\end{array}$ & $\begin{array}{l}\text { NVO 10:0 2O RT-2.4 } \\
\text { NVO 10:0 2O RT-3.7 } \\
\text { HOTrE RT-4.3 } \\
\text { FFA 20:1 RT-10.4 } \\
\text { FFA 22:1 RT-11.7 } \\
\text { NVO 22:4 2O RT-9.3 } \\
\text { NVO 22:4 3O RT-6.4 } \\
\text { NVO 22:3 3O RT-6.1 }\end{array}$ & 16.9 & 2.1 & 1.8 \\
\hline
\end{tabular}


Table 17. Results of permutational multivariate analysis of variance of dissolved lipidome structure with respect to the core supplemental variables sampled along the DYEatom cruise, using ADONIS (permutations = 999, method= Euclidean). Asterisk denotes significant correlation between supplemental variable and dissolved lipidome structure $(\mathrm{p}<0.1)$.

\begin{tabular}{lrrrrrr}
\hline & $d . f$. & \multicolumn{1}{c}{$S S$} & \multicolumn{1}{c}{$M S$} & \multicolumn{1}{c}{$F$} & $R^{2}$ & \multicolumn{1}{c}{$P$} \\
\hline Phaeophytin & 1 & 0.3282 & 0.3282 & 1.94357 & 0.14828 & $* 0.073$ \\
bSi & 1 & 0.22545 & 0.22545 & 1.33505 & 0.10185 & 0.233 \\
$\mathrm{PO}_{4}{ }^{3-}$ & 1 & 0.3021 & 0.3021 & 1.78898 & 0.13648 & 0.101 \\
$\mathrm{dSi}$ & 1 & 0.16012 & 0.16012 & 0.94822 & 0.07234 & 0.49 \\
Nitrogen & 1 & 0.21556 & 0.21556 & 1.27648 & 0.09738 & 0.282 \\
Chlorophyll a & 1 & 0.19505 & 0.19505 & 1.15507 & 0.08812 & 0.336 \\
Total Chlorophyll & 1 & 0.28035 & 0.28035 & 1.66021 & 0.12666 & 0.13 \\
Residuals & 3 & 0.5066 & 0.16887 & 0.22888 & & \\
Total & 10 & 2.21344 & & & &
\end{tabular}


Table 18. Non-volatile oxylipins observed in northern Adriatic and correlated with decreased hatching success of copepods (Ianora et al

2015). The diatom species from which each molecule was originally extracted is also noted: Skeletonema marinoi (Sm; Fontana et al., 2007),

Chaetoceros socialis (Cs; Fontana et al., 2007), Chaetoceros affinis (Ca; Fontana et al., 2007), Thalassiosira rotula (Tr; d'Ippolito et al.,

2005), Pseudo-nitzchia delicatissima (Pd; d'Ippolito et al., 2009), Skeletonema pseudocostatum (Sp; Barreiro et al., 2011). Four levels of

annotation are given for each molecular species. The lipidomic pipeline in this study automatically annotates to the structural level.

Structural isomers of these compounds were observed in the DYEatom cruise dissolved lipidome. Many of these matched were compounds

that were annotated to the function group level, representing bioactive compounds in the environment.

\begin{tabular}{|c|c|c|c|c|c|c|}
\hline $\begin{array}{l}\text { Diatom } \\
\text { species }\end{array}$ & Chiral level & Positional level & $\begin{array}{l}\text { Functional } \\
\text { group level }\end{array}$ & $\begin{array}{l}\text { Observed in } \\
\text { this study }\end{array}$ & Structural level & $\begin{array}{l}\text { Observed in } \\
\text { this study }\end{array}$ \\
\hline $\mathrm{Sm}, \mathrm{Tr}$ & (7E)-9-hydroxy-7-hexaenoic acid & 9-HME & HHME & & NVO C16:1 10 & $\mathrm{x}$ \\
\hline $\mathrm{Sm}, \mathrm{Tr}$ & (7E)-9-oxo-7-hexadecaenoic acid & 9-KHME & KHME & $\mathrm{x}$ & NVO C16:2 10 & $\mathrm{x}$ \\
\hline $\mathrm{Sm}, \mathrm{Tr}$ & $\begin{array}{l}(6 \mathrm{Z}, 9 \mathrm{~S}, 10 \mathrm{E}, 12 \mathrm{Z})-9-\text { hydroxy-6,10,12- } \\
\text { hexadecatrienoic acid }\end{array}$ & 9-HHTrE & HHTrE & & NVO C16:3 10 & $\mathrm{x}$ \\
\hline $\mathrm{Sm}$ & $\begin{array}{l}(6 \mathrm{Z}, 9 \mathrm{~S}, 10 \mathrm{E}, 12 \mathrm{Z})-9 \text {-hydroperoxy-6,10,12- } \\
\text { hexadecatrienoic acid }\end{array}$ & 9-HpHTrE & HpHTrE & & NVO C16:3 20 & $\mathrm{x}$ \\
\hline $\mathrm{Sm}$ & $\begin{array}{l}(6 \mathrm{Z}, 9 \mathrm{~S}, 10 \mathrm{E}, 12 \mathrm{Z}, 15 \mathrm{E})-9 \text {-hydroperoxy-6,10,12,15- } \\
\text { hexadecatetraenoic acid }\end{array}$ & 9-НрНТЕ & HрHTE & $\mathrm{x}$ & NVO C16:4 20 & $\mathrm{x}$ \\
\hline $\mathrm{Sm}$ & $\begin{array}{l}\text { 6Z,9RS,10SR,11SR,12Z)-11-hydroxy-9,10- } \\
\text { epoxyhexadeca-6,12-dienoic acid }\end{array}$ & 11,9-HepHDE & HepHDE & $\mathrm{x}$ & NVO C16:3 20 & $\mathrm{x}$ \\
\hline $\begin{array}{l}\mathrm{Sm}, \\
\mathrm{Pd}, \mathrm{Sp}\end{array}$ & $\begin{array}{l}\text { (5Z,8Z,11Z,13E,15S,17Z)-15-hydroxy-5,8,11,13,17- } \\
\text { eicosapentaenoic acid }\end{array}$ & 15-HEPE & HEPE & $\mathrm{x}$ & NVO C20:5 10 & $\mathrm{x}$ \\
\hline $\mathrm{Sm}$ & $\begin{array}{l}\text { (5Z,8Z,11Z,13E,15S,17Z)-15-hydroperoxy- } \\
5,8,11,13,17 \text {-eicosapentaenoic acid }\end{array}$ & 15-HpEPE & HpEPE & & NVO C20:5 20 & $\mathrm{x}$ \\
\hline $\mathrm{Sm}$ & $\begin{array}{l}(5 \mathrm{R}, 6 \mathrm{E}, 8 \mathrm{Z}, 11 \mathrm{Z}, 14 \mathrm{Z}, 17 \mathrm{Z})-5 \text {-hydroxy-6,8,11,14,17- } \\
\text { eicosapentaenoic acid }\end{array}$ & 5-HEPE & HEPE & $\mathbf{x}$ & NVO C20:5-10 & $\mathrm{x}$ \\
\hline $\mathrm{Sm}$ & $\begin{array}{l}\text { (5R,6E,8Z,11Z,14Z,17Z)-5-hydroperoxy- } \\
6,8,11,14,17 \text {-eicosapentaenoic acid }\end{array}$ & 5-HpEPE & HpEPE & & NVO C20:5 20 & $\mathrm{X}$ \\
\hline
\end{tabular}


Table 18. (continued)

\begin{tabular}{|c|c|c|c|c|c|c|}
\hline $\begin{array}{l}\text { Diatom } \\
\text { species }\end{array}$ & Chiral level & Positional level & $\begin{array}{l}\text { Functional } \\
\text { group level }\end{array}$ & $\begin{array}{l}\text { Observed in } \\
\text { this study }\end{array}$ & Structural level & $\begin{array}{l}\text { Observed in } \\
\text { this study }\end{array}$ \\
\hline $\begin{array}{c}\mathrm{Sm}, \\
\mathrm{Tr}, \mathrm{Pd} \\
\mathrm{Sc}\end{array}$ & $\begin{array}{l}\text { (5Z,8Z,11Z,13RS,14RS,15SR,17Z)-13-hydroxy- } \\
\text { 14,15-epoxyeicosa-5,8,11,17-tetraenoic acid }\end{array}$ & 13,14-HepETE & HepETE & & NVO C20:5 20 & $\mathrm{x}$ \\
\hline $\mathrm{Sm}$ & $\begin{array}{l}\text { (5Z,7E,9S,11Z,14Z,17Z)-9-hydroxy-5,7,11,14,17- } \\
\text { eicosapentaenoic acid }\end{array}$ & 9-HEPE & HEPE & $\mathrm{x}$ & NVO C20:5 10 & $\mathrm{x}$ \\
\hline $\mathrm{Sm}$ & $\begin{array}{l}(5 Z, 7 \mathrm{E}, 9 \mathrm{~S}, 11 \mathrm{Z}, 14 \mathrm{Z}, 17 \mathrm{Z}) \text {-9-hydroperoxy- } \\
\text { 5,7,11,14,17-eicosapentaenoic acid }\end{array}$ & 9-HpEPE & HpEPE & $\mathrm{x}$ & NVO C20:5 20 & $\mathrm{x}$ \\
\hline Cs & $\begin{array}{l}\text { (5Z,7SR,8RS,9SR,11Z,14Z,17Z)-7-hydroxy-8,9- } \\
\text { epoxy-5,11,14,17-eicosatetraenoic acid }\end{array}$ & 7,8-НерЕTE & HepETE & & NVO C20:5 20 & $\mathrm{x}$ \\
\hline Cs & $\begin{array}{l}\text { 5Z,8Z,11Z,15E,17Z)-14-hydroxy-(5,8,11,15,17)- } \\
\text { eicosapentaenoic acid }\end{array}$ & 14-HEPE & HEPE & $\mathrm{x}$ & NVO C20:5 10 & $\mathrm{x}$ \\
\hline $\mathrm{Ca}$ & $\begin{array}{l}\text { 5Z,8Z,11Z,15E,17Z)-14-hydroperoxy-(5,8,11,15,17)- } \\
\text { eicosapentaenoic acid }\end{array}$ & 14-HpEPE & HpEPE & $\mathrm{x}$ & NVO C20:5 20 & $\mathrm{x}$ \\
\hline $\mathrm{Ca}$ & $\begin{array}{l}\text { (5Z,8Z,11Z,14SR,15RS,16SR,17Z)-16-hydroxy- } \\
\text { 14,15-epoxy-5,8,11,17-eicosatetraenoic acid }\end{array}$ & 16,14-HepETE & HepETE & & NVO C20:5 20 & $\mathrm{x}$ \\
\hline $\mathrm{Pd}, \mathrm{Sp}$ & 15-oxo-5Z,9E,11E,13E-pentadecatetraenoic acid & 15-охо-РТА & охо-РTA & & NVO C15:5 10 & \\
\hline
\end{tabular}


Table 19. Regression of total RNA viral reads, grazing, and metrics of nutrient state against phaeophytin for samples where the dissolved lipidome was sampled along the DYEatom cruise. Bold values denote significance $\mathrm{F}<0.1$.

\begin{tabular}{lcc}
\hline $\begin{array}{l}\text { Supplemental } \\
\text { variable }\end{array}$ & $\mathrm{R}^{2}$ & Significance F \\
\hline Total Viral Reads & 0.67 & $\mathbf{0 . 0 1 1}$ \\
Grazing Rate & 0.96 & $\mathbf{0 . 0 2 3}$ \\
N:P & 0.14 & 0.256 \\
Nitrogen & 0.18 & 0.194 \\
SRP & 0.09 & 0.357 \\
dSi & 0.00 & 0.91 \\
Si-stress & 0.28 & $\mathbf{0 . 0 9 1}$
\end{tabular}




\section{Chapter 5}

Conclusions 
For nearly two decades, oxylipin infochemical signaling has been implicated in the marine carbon cycle through culture studies, mesocosm experiment, and a handful of field studies (Andreou et al., 2009; Bartual and al, 2014; Ianora et al., 2015; Ribalet et al., 2014; Vidoudez et al., 2011). While these studies focused on copepods and eukaryotic phytoplankton, the impact of oxylipins on major players in the microbial loop, particle associated bacteria and microzooplankton, as well as, the diversity of oxylipin compounds in situ has remained largely unknown. Filling in these gaps is imperative for fully understand the ecological importance of oxylipin infochemical signaling in the ocean, especially considering the wide range of bioactive responses mediated by oxylipins and the importance of particle associated bacteria and microzooplankton to energy transfer in the ocean (Azam et al., 1994; Calbet, 2004; Grossart et al., 2007; Schmoker et al., 2013). The research undertaken in this thesis addressed these gaps in our knowledge by pairing hypothesis driven field and culture experiments with oxylipin profiling in situ.

The link between oxylipins and particle associated bacteria was queried in natural populations across the North Atlantic while also surveying PUA concentrations on sinking particles. Sinking particles were hotspots for oxylipin infochemical signaling in the ocean with micromolar concentrations of decadienal, a well-studied oxylipin implicated in decreased copepod reproductive success and diatom stress surveillance. We also gained improved understanding of the dose-dependent response of particle associated bacteria to PUAs, unveiling a stimulatory response at sub-lethal concentrations of $10 \mu \mathrm{M}$. As diatom blooms decline, accumulation of micromolar PUA concentrations could result in enhanced remineralization of diatom derived organic matter in the upper ocean, contributing to nutrient recycling and carbon flux attenuation. Exposure to PUAs also altered the particle associated bacteria community structure, suggesting that oxylipins produced by diatoms my influence the succession of heterotrophic bacteria in the ocean. There are further intricacies to explore including the mechanisms by which particle associated bacteria are stimulated by PUAs, depth resolved PUA concentration on sinking particles, the impact of PUAs on biogenic Si remineralization, and whether particle associate bacteria communities in different ocean basins respond the same to PUA amendment. 
The impact of oxylipins produced by diatoms on microzooplankton grazing was investigated in two ways, amendment experiments were conducted in the presence of exogenous oxylipins and de novo oxylipin production was assessed as Pt and a chronically stressed transgenic strain of Pt (PtNOA) were grazed by microzooplankton grazing. The exogenous oxylipin experiments elucidated the impact of PUAs and non-volatile oxylipins on microzooplankton grazing, an interaction previously only alluded to in the literature (Stoecker, 2015). Furthermore the de novo approach revealed that microzooplankton grazed healthy diatoms faster than oxidatively stressed diatoms, even though healthy diatoms exuded a more robust lipidome than stressed diatoms. This was attributed to upregulation of small unknown molecules in grazed PtNOA and the dose dependence of oxylipin infochemical signaling. The lipidomic data revealed several novel compounds produced by $\mathrm{Pt}$ that are ripe for further investigation.

To determine the suit of oxylipin compounds dissolved in situ a streamlined, analytically broad method was employed without need for sonication or derivatization. Canonical diatom fatty acids and oxylipins dominated the dissolved lipidome across the DYEatom cruise track. The variation in the relative abundance of these molecules and overall structure of the dissolve lipidome were associated with bloom demise and RNA viral abundance. Several potentially bioactive molecules were annotated within the dissolved lipidome, exhibiting higher abundances at stations with high concentrations of phaeophytin and RNA viral metatranscriptomic reads. Microzooplankton grazing was not implicated as an important factor for oxylipin distributions in situ, potentially due to the bioactivity of the aforementioned molecules deterring grazing. Virally induced oxylipin production may also function to decrease virus burst size by aggregating infected cells and exporting them to depth before lysis (Mojica and Brussaard, 2014). The biogeochemical implications of virally induced oxylipin production are broad and warrant further investigation. Furthermore, several novel molecules were observed in situ broaden our perspective on avenues of infochemical exchange in the ocean. Improvements on lipidomic technique utilized in this thesis will give us a clearer view of oxylipin production in situ and oxylipins to target for future bioactivity assays.

Overall, the data put forth in this thesis furthers the assertion that infochemical signaling via oxylipins is an important component of carbon flux through upper ocean ecosystems. 
Infochemical signaling molecules were abundant on sinking particles and wide spread in the dissolved pool of the upper ocean during bloom conditions, exerting influence on both particle associated bacteria and microzooplankton.

\section{References}

Andreou, A., Brodhun, F., Feussner, I., 2009. Biosynthesis of oxylipins in non-mammals. Progress in Lipid Research 48, 148-170.

Azam, F., Smith, D.C., Steward, G.F., Hagstrom, A., 1994. Bacteria-organic matter coupling and its significance for oceanic carbon cycling. Microbial Ecology 28, 167-179.

Bartual, A., al, e., 2014. Polyunsaturated Aldehydes from Large Phytoplankton of the Atlantic Ocean Surface (42 N to $33 \mathrm{~S}$ ). Marine drugs 12, 682-699.

Calbet, A.a.L.M.R., 2004. Phytoplankton growth, microzooplankton grazing, and carbon cycling in marine systems. Limnology and Oceanography 49, 51--57.

Grossart, H.-P., Tang, K.W., Kiorboe, T., Ploug, H., 2007. Comparison of cell-specific activity between free-living and attached bacteria using isolates and natural assemblages. Fems Microbiology Letters 266, 194-200.

Ianora, A., Bastianini, M., Carotenuto, Y., Casotti, R., Roncalli, V., Miralto, A., Romano, G., Gerecht, A., Fontana, A., Turner, J.T., 2015. Non-volatile oxylipins can render some diatom blooms more toxic for copepod reproduction. Harmful Algae 44, 1-7.

Mojica, K.D.A., Brussaard, C.P.D., 2014. Factors affecting virus dynamics and microbial host-virus interactions in marine environments. Fems Microbiology Ecology 89, 495-515.

Ribalet, F., Bastianini, M., Vidoudez, C., Acri, F., Berges, J., Ianora, A., Miralto, A., Pohnert, G., Romano, G., Wichard, T., Casotti, R., 2014. Phytoplankton Cell Lysis Associated with Polyunsaturated Aldehyde Release in the Northern Adriatic Sea. PLoS ONE 9, e85947.

Schmoker, C., Hernandez-Leon, S., Calbet, A., 2013. Microzooplankton grazing in the oceans: impacts, data variability, knowledge gaps and future directions. Journal of Plankton Research 35, 691-706.

Stoecker, D.K., Nejstgaard, J.C., Mashusoodhanan, R., Pohnert, G., Wolfram, S., Jakobsen, H.H., Šulčius, S., and Larsen, A., 2015. Underestimation of microzooplankton grazing in dilution experiments due to inhibition of phytoplankton growth. Limnology and Oceanography 60, 1426--1438.

Vidoudez, C., Casotti, R., Bastianini, M., Pohnert, G., 2011. Quantification of dissolved and particulate polyunsaturated aldehydes in the Adriatic Sea. Marine drugs 9, 500-513. 


\section{Appendix A}

List of abbreviations for free fatty acids and non-volatile oxylipins 
List of abbreviations for free fatty acids (FFA) and non-volatile oxylipins (NVO) used throughout this thesis. Numbering system corresponds to Figure 1 in Chapter 3 and Chapter 4. Compounds that were represented by one or more positional isomers in the authentic standard suite are in bold.

\begin{tabular}{|c|c|c|c|}
\hline Abbreviation & Full name & $\begin{array}{l}\text { Structural } \\
\text { annotation }\end{array}$ & No. \\
\hline HpDA & Hydroperoxy dodecanoic acid & NVO 12:0 20 & \\
\hline HpDMA & Hydroperoxy dodecaenoic acid & NVO 12:1 2O & \\
\hline HpTA & Hydroperoxy tetradecanoic acid & NVO 14:0 2O & \\
\hline HHA & Hydroxy hexanoic acid & NVO 16:0 10 & \\
\hline HpHA & Hydroperoxy hexanoic acid & NVO 16:0 20 & \\
\hline HpHME & Hydroperoxy hexadecenoic acid & NVO 16:1 20 & \\
\hline HHME & Hydroxy hexadecenoic acid & NVO 16:1 1O & \\
\hline KHME & Keto hexadecenoic acid & NVO 16:2 10 & \\
\hline HpHDE & Hydroperoxy hexadecadienoic & NVO 16:2 2O & \\
\hline HHDE & Hydroxy hexadecadienoic acid & NVO 16:2 10 & \\
\hline KHDE & Keto hexadecadienoic acid & NVO 16:3 10 & \\
\hline HTrE & Hexadecatrienoic acid & FFA $16: 3$ & 1 \\
\hline HpHTrE & Hydroperoxy hexadecatrienoic acid & NVO 16:3 2O & $2 \& 3$ \\
\hline HepHDE & Hydroxy epoxy hexadecadienoic acid & NVO 16:3 20 & 7 \\
\hline HHTrE & Hydroxy hexadecatrienoic acid & NVO 16:3 10 & $8 \& 9$ \\
\hline KHTrE/oxo-HTE & Keto hexadecatrienoic acid & NVO 16:4 10 & 10 \\
\hline HTE & Hexadecatetraenoic acid & FFA $16: 4$ & 11 \\
\hline HpHTE & Hydroperoxy hexadecatetraenoic acid & NVO 16:4 2O & $12 \& 13$ \\
\hline HHTE & Hydroxy hexadecatetraenoic acid & NVO 16:4 10 & $15 \& 16$ \\
\hline KHTE/oxo-ETE & Keto hexadecatetraenoic acid & NVO 16:5 10 & 17 \\
\hline HODE & Hydroxy octadecadienoic acid & NVO 18: 210 & \\
\hline KOME & Keto octadecaenoic acid & NVO 18:2 10 & \\
\hline HpODE & Hydroperoxy octadecadienoic acid & NVO 18:2 20 & \\
\hline KODE & Keto octadecadienoic acid & NVO 18:3 10 & \\
\hline HOTrE & Hydroxy octadecatrienoic acid & NVO $18: 310$ & \\
\hline HpOTrE & Hydroperoxy octadecatrienoic acid & NVO 18:3 20 & \\
\hline KOTrE/oxo-OTrE & Keto octadecatrienoic acid & NVO 18:4 10 & \\
\hline HpEA & Hydroperoxy eicosanoic acid & NVO 20:0 20 & \\
\hline diHEME & Dihydroxy eicosaenoic acid & NVO 20:1 20 & \\
\hline HETrE & Hydroxy eicosatrienoic acid & NVO 20:3 10 & \\
\hline $\mathbf{A A}$ & Arachidonic acid/eicosatetraenoic acid & FFA 20:4 & 18 \\
\hline HpETE & Hydroperoxy eicosatetraenoic acid & NVO 20:4 20 & $19 \& 20$ \\
\hline HETE & Hydroxy eicosatetraenoic acid & NVO 20:4 10 & 23 \\
\hline diHETE & Dihydroxy eicosatetraenoic acid & NVO 20:4 20 & \\
\hline EpETE & Epoxy eicosatetraenoic acid & NVO 20:5 10 & \\
\hline EPA & Eicosapentaenoic acid & FFA 20:5 & 26 \\
\hline HpEPE & Hydroperoxy eicosapentaenoic acid & NVO 20:5 20 & $27-33$ \\
\hline HEPE & Hydroxy eicosapentaenoic acid & NVO 20:5 10 & $40,48,44, \& 51$ \\
\hline HepETE & Hydroxy epoxy eicosatetraenoic acid & NVO 20:5 20 & $41-43,45,46, \& 50$ \\
\hline diHEPE & Dihydroxy eicosapentaenoic acid & NVO 20:5 20 & \\
\hline diDoME & Dihydroxy docosaenoic acid & NVO 22:1 20 & \\
\hline diDoDE & Dihydroxy docosadienoic acid & NVO 22:2 20 & \\
\hline HpDoTE & Hydroperoxy docosatetraenoic acid & NVO 22:4 20 & \\
\hline
\end{tabular}




\begin{tabular}{|l|l|l|l|}
\hline DHA & Docosahexaenoic acid & FFA 22:6 & 53 \\
\hline HpDoHE & Hydroperoxy docosahexaenoic acid & NVO 22:6 2O & $54 \& 55$ \\
\hline HDoHE & Hydroxy docosahexaenoic acid & NVO 22:6 1O & $57 \& 56$ \\
\hline HepDoPE & Hydroxy epoxy docosapentaenoic acid & NVO 22:6 2O & $58 \& 59$ \\
\hline
\end{tabular}




\section{Appendix B}

Comparison of solid phase extraction methods for sampling dissolved oxylipins and PUAs in seawater. 


\section{Recovery of PUAs}

Two different solid phase extraction (SPE) cartridges were tested to determine which should be used for sampling dissolved PUAs in seawater. A PUA stock solution containing $100 \mathrm{mM}$ of heptadienal, octadienal, and decadienal each was prepared in methanol. Two $10 \mu \mathrm{M}$ PUA solutions were made from the stock solution, one in $20 \mathrm{~mL}$ of filtered sea water and another in $20 \mathrm{~mL}$ of methanol. C18 (Thermo) and HLB (Waters) SPE cartridges were preconditioned according to the manufactures' instructions. A $10 \mathrm{~mL}$ aliquot of the PUA amended sea water was filtered onto each SPE column. The columns were stored in whirlpak bags at $-80 \mathrm{C}$ for 24 hours to simulate sample storage at sea. The SPE cartridges were removed from the freezer and allowed to thaw over 5 minutes. Then the PUAs were eluted from the cartridges with $4 \mathrm{~mL}$ methanol into a pre-combusted culture tube that was pretreated with BHT, an antioxidant added to prevent auto-oxidation. The samples were transferred to HPLC vials and blown down with $\mathrm{N}_{2}$, re-dissolved in $0.5 \mathrm{~mL}$ methanol, and capped under argon before analysis. Complete recovery of the SPE samples would yield extracts with a concentration of $200 \mu$ M. Samples were analyzed on the LCQ-Fleet instrument by direct infusion, as this phase of method development predated our high resolution-accurate mass-mass spectrometric (HRAM-MS) method. The 10uM stock solution was also directly infused into the instrument. Decadienal, octadienal, and heptadienal were prominent masses in the MS from the $10 \mu \mathrm{M}$ PUA stock and the extract from the HLB column (Figure 1A and 1B). However, PUAs were only about $20 \%$ of the signal in the extract from the $\mathrm{C} 18$ column (Figure 1C). After accounting for the difference in the concentration of the stock and the SPE samples, C18 and HLB columns were found to have 15\% and $42 \%$ recovery of PUAs (Table 1). The intensity of signal in the 10uM PUA solution in $\mathrm{MeOH}$ and the HLB extract as well as the recovery calculation can be found in Table 1 . 

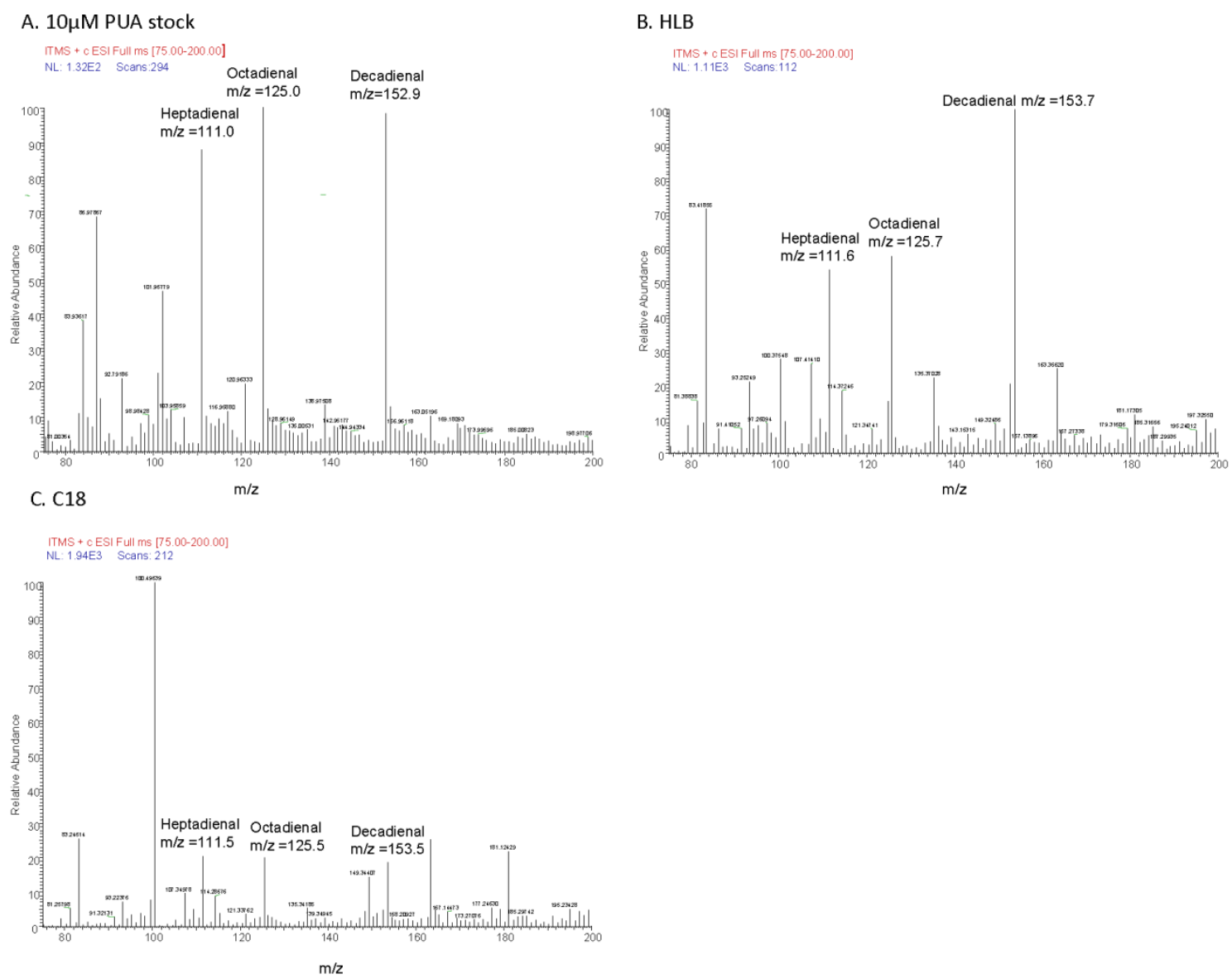

Figure 1. Mass spectra from direct injection of A) $10 \mu \mathrm{M}$ PUA stock, B) HLB extract, and C) C18 extract collected by direct injection at $20 \mu \mathrm{L} \mathrm{min}^{-1}$ into a LCQ-Fleet instrument using electrospray ionization (ESI).

\begin{tabular}{|c|c|c|c|c|c|}
\hline Sample & $\begin{array}{l}\text { Observed } \\
\text { Signal }\end{array}$ & $\begin{array}{l}\text { Concentration } \\
\text { assuming } \\
100 \% \text { recovery }\end{array}$ & $\begin{array}{l}\text { Correction } \\
\text { Factor }\end{array}$ & $\begin{array}{l}\text { Corrected } \\
\text { Signal }\end{array}$ & $\%$ Recovery \\
\hline $\begin{array}{l}10 \mu \mathrm{M} \text { PUA } \\
\text { stock }\end{array}$ & $1.32 \mathrm{E} 2$ & -- & 20 & $2.64 \mathrm{E} 3$ & -- \\
\hline HLB & $1.1 \mathrm{E} 3$ & $200 \mu \mathrm{M}$ & -- & $1.1 \mathrm{E} 3$ & $42 \%$ \\
\hline $\mathrm{C} 18$ & $1.94 \mathrm{E} 3$ & $200 \mu \mathrm{M}$ & $\begin{array}{l}0.2 \text { (PUAs } \\
\text { were } 20 \% \text { of } \\
\text { the signal) }\end{array}$ & $3.9 \mathrm{E} 2$ & $15 \%$ \\
\hline
\end{tabular}




\section{Recovery of free fatty acids and non-volatile oxylipins}

The two SPE cartridges were also tested to determine which should be used for sampling dissolved fatty acids and non-volatile oxylipins in seawater. C18 and HLB columns were tested by extracting oxylipins from an experiment aimed at biosynthesizing hydroperoxy eicosatetraenoic acid (HpETE) from eicosatetraenoic acid (also known as arachidonic acid, AA). In a 50mL solution $25 \mathrm{mg}$ of AA was added to soybean lipoxygenase and bubbled with oxygen. The C18 (Thermo) and HLB (Waters) SPE cartridges were preconditioned according to the manufactures' instructions. After allowing the lipoxygenation reaction to take place for 20 minutes, $4 \mathrm{~mL}$ of reaction was filtered onto the HLB column and $6 \mathrm{~mL}$ was filtered onto the $\mathrm{C} 18$. The columns were stored in whirlpak bags at $-80 \mathrm{C}$ for 24 hours to simulate sample storage at sea. The SPE cartridges were removed from the freezer and allowed to thaw over 5 minutes. Then the PUAs were eluted from the cartridges with $0.5 \mathrm{~mL}$ of methanol into a pre-combusted culture tube that was pretreated with BHT, an antioxidant added to prevent auto-oxidation. The samples were transferred to HPLC vials and blown down with $\mathrm{N}_{2}$, re-dissolved in $0.5 \mathrm{~mL}$ methanol, and capped under argon before analysis. Assumming 100\% recovery the combined concentration of AA and HpETE in the HLB sample was $13.8 \mathrm{mM}$ and in the C18 samples it was $20.6 \mathrm{mM}$. The HLB extract was diluted to $13.8 \mu \mathrm{M}$ and $\mathrm{C} 18$ extract was diluted to $20.6 \mu \mathrm{M}$ so that they couls be compared to a $10 \mu \mathrm{M}$ solution of AA in methanol. The extracted samples were analyzed by HRAM-MS on the Thermo Exactive Plus by direct infusion. A $10 \mu \mathrm{M}$ solution of AA in methanol was also infused into the instrument. After accounting for the difference in the concentrations of the $\mathrm{C} 18$ and HLB extracts, the two columns were found to have nearly complete recovery of non-volatile oxylipins (Figure 2A-C; Table 2). However, the HLB column displayed better recovery than the C18 column for PUAs (Section 1) and was adopted as the SPE column for dissolved lipidome analysis. HLB columns are unique in that they are specific for hydrophilic and lipophilic compounds allowing us to target IPL, oxylipins, and PUAs. 


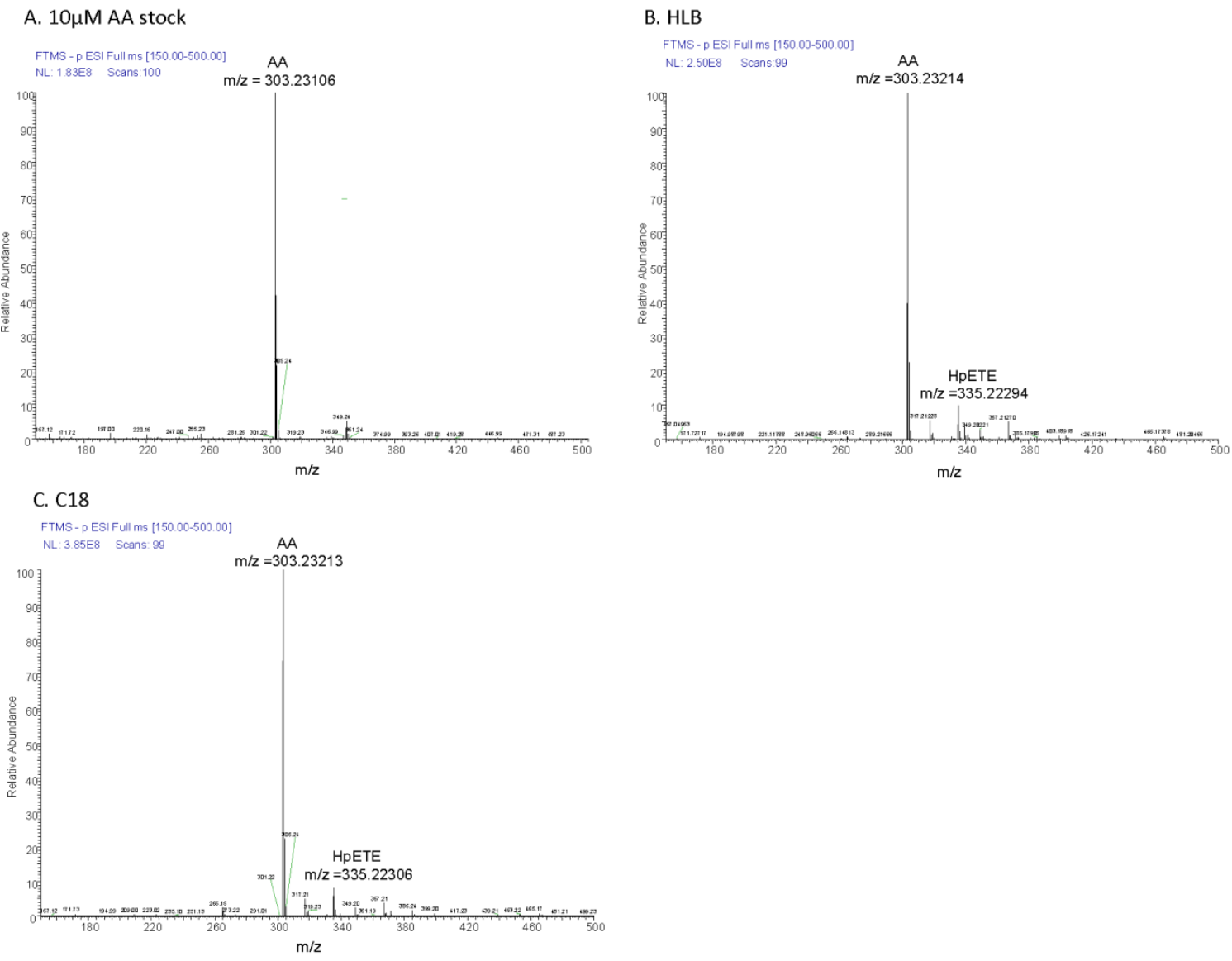

Figure 2. Mass spectra of A) $10 \mu \mathrm{M}$ Arachidonic acid solution in methanol, B) HLB extract, and C) $\mathrm{C} 18$ extract collected by direct injection at $20 \mu \mathrm{L} \mathrm{min}^{-1}$ into a Thermo Exactive Plus instrument using electrospray ionization (ESI).

\begin{tabular}{llllllc}
\hline \multicolumn{6}{l}{ Table 2. Recovery of non-volatile oxylipins from HLB and C18 solid phase extraction columns } \\
\hline Sample & $\begin{array}{l}\text { Observed } \\
\text { Signal }\end{array}$ & $\begin{array}{l}\text { Concentration } \\
\text { assuming } \\
100 \% \text { recovery }\end{array}$ & Dilution & $\begin{array}{l}\text { Correction } \\
\text { Factor }\end{array}$ & $\begin{array}{l}\text { Corrected } \\
\text { Signal }\end{array}$ & \% Recovery \\
\hline $\begin{array}{l}10 \mu \mathrm{M} \text { AA } \\
\text { stock }\end{array}$ & $1.83 \mathrm{E} 8$ & -- & -- & 2.06 & $3.77 \mathrm{E} 8$ & -- \\
HLB & $2.5 \mathrm{E} 8$ & $13.8 \mathrm{mM}$ & $13.8 \mu \mathrm{M}$ & 1.5 & $3.75 \mathrm{E} 8$ & $99.5 \%$ \\
C18 & $3.84 \mathrm{E} 3$ & $20.6 \mathrm{mM}$ & $20.6 \mu \mathrm{M}$ & -- & $3.84 \mathrm{E} 8$ & $102 \%$
\end{tabular}


Appendix C

Abiotic and exoenzymatic degradation of PUAs in natural seawater. 


\section{Degradation rates of PUAs}

Degradation rates of PUAs had not previously been investigated. We were interested to know how stable these compounds are in the environment and whether we would expect to be able to measure them in situ. On the AEx1103 cruise in the Sargasso Sea, water was collected from the deep chlorophyll max and processed in three ways before adding a PUA cocktail of heptadienal, octadienal, and decadienal in a final concentration of 500nM. The three different conditions were: Unfiltered SW (WSW), filtered SW (FSW), and filtered SW spiked with proteinase K. The SW filtered through a $0.2 \mu \mathrm{m}$ filter resulted in all cells being removed and only exoenzymes being present. The proteinase $\mathrm{K}$ spiked filtered SW served as an abiotic condition as all proteins were degraded. I aliquoted $20 \mathrm{ml}$ of each condition into $36-40 \mathrm{ml}$ EPA vials, incubated in the dark at approximately $23^{\circ} \mathrm{C}$, and sacrificed three EPA vials at each time point for each condition. I used a first order rate equation for the abiotic degradation rate and a first order two component rate equation for the FSW and WSW degradation rates as abiotic degradation was also occurring.

Abiotic degradation became faster as chain length increased (Figure 1). Very similar to what Hmelo and Van Mooy (2009) observed with 3-oxo substituted AHL. Whereas, exoenzymatic degradation became slower with an increase in chain length, suggesting increased steric hindrances in active sites of the enzymes. Degradation rates in the whole seawater treatments were confounded by production of PUAs (data not shown). The degradation rates observed suggest that the turnover time of PUAs is on the order of hours. Thus PUAs observed in the environment must have been produced recently.

\section{References}

Hmelo and Van Mooy (2009) Kinetic constraints on acylated homoserine lactone-based quaorum sensing in marine environments. Aqua Microb Ecol 54:127-133. 


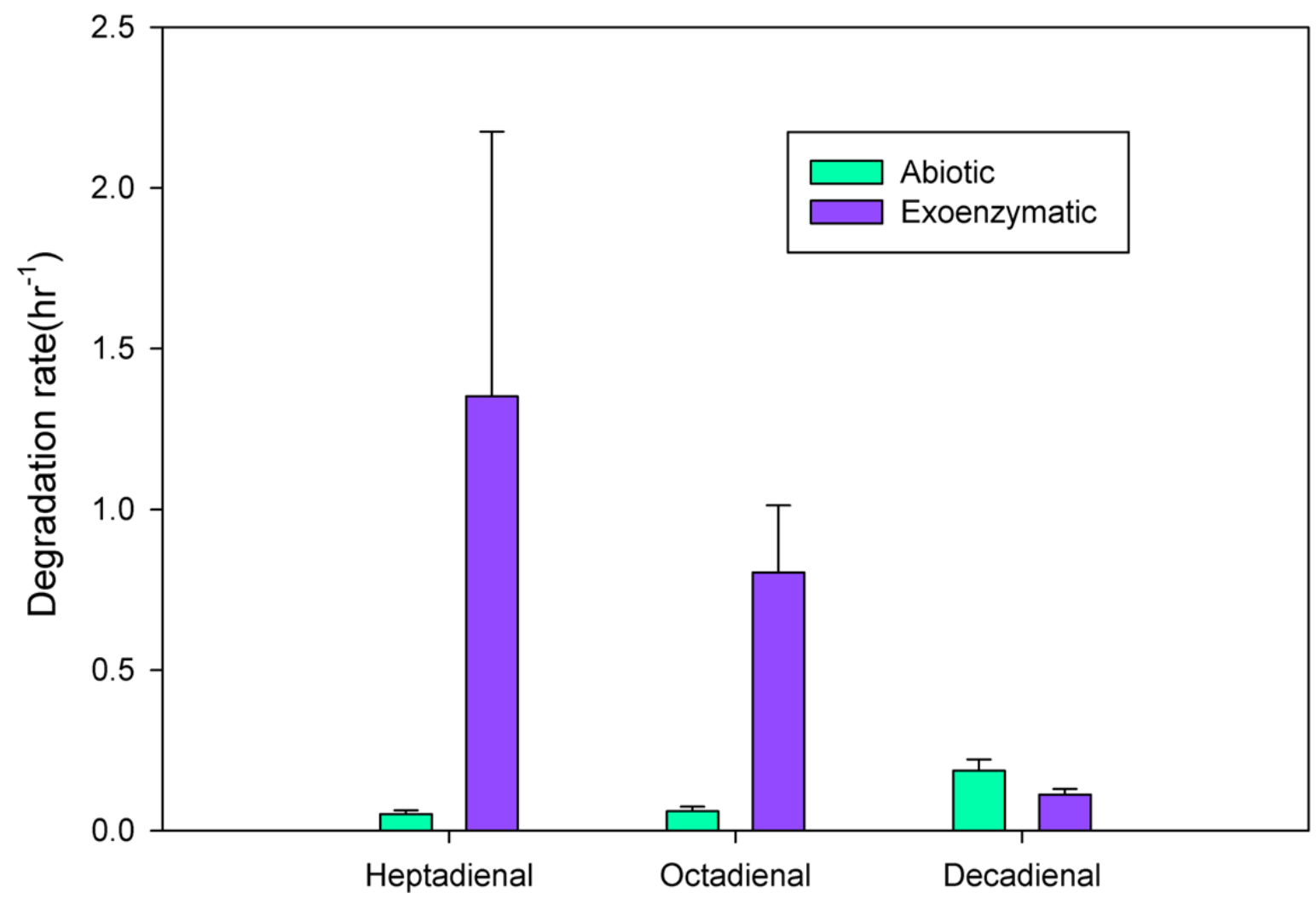

Figure 1. Degradation rates $\left(\mathrm{hr}^{-1}\right)$ for heptadienal octadienal, and decadienal in two conditions. A first order rate equation for the abiotic degradation rate and a first order two component rate equation for the exoenzymatic degradation rate since abiotic degradation was also occurring. 


\section{Appendix D}

The influence of PUAs on biogenic Si remineralization on sinking particles exported from Si-stressed phytoplankton communities. 


\section{Amendment experiments exposing sinking particles to varying concentrations of PUAs}

During the DYEatom cruise in the California Current System, June 27th -July 6th 2013, amendment experiments were conducted to determine how oxylipins impact the recycling of biogenic silica (BSi) by particle associated communities. Sinking particles were collected from

three surface-tethered net traps deployments (6-8 hours at 50m) at Stations 2, 6, and 7 presented in Chapter 4 of this thesis. Sinking particles were incubated with varying concentrations of a PUA cocktail (heptadienal, octadienal, and decadienal). Four treatments were set up with final concentration of $0,1 \mu \mathrm{M}, 10 \mu \mathrm{M}$, or $100 \mu \mathrm{M}$ PUAs. Triplicates of each treatment were incubated in the dark at in situ temperature for 24 hours. Samples were collected for bSi at $\mathrm{t}=0$ and bSi and enzymatic activity at $\mathrm{t}=$ final.

We found that the response of particle associated bacteria to PUAs differed depending on the nutrient state of the environment. In the Si-stressed environment, high doses of PUAs, 100 $\mu \mathrm{M}$, resulted in less BSi remaining in the incubation after $24 \mathrm{hrs}$ compared to the control, suggesting enhanced BSi dissolution (Figure 1B). One might expect that this is due to increases peptidase activity due the relationship between peptidase activity and BSi dissolution observed by Bidle and Azam (2000). However, upon addition of PUAs the activities of the three peptidases that we measured were significantly lower than the control and alkaline phosphatase activity was actually enhanced (Figure 1A). Perhaps alkaline phosphatases play a similar role to peptidases in removing organics from the surface of diatom frustules and increasing BSi dissolution in under saturated waters. At the Si-replete stations, Apase activity exhibits a dose dependent response to PUAs with enhanced activity at moderate concentrations of PUAs, 10 $\mu \mathrm{M}$, and significantly lower activity at high concentrations, $100 \mu \mathrm{M}$ (Figure 1C). Peptidase activity is depressed by the addition of PUAs at concentrations higher than 10uM. However, the bSi concentrations remain the same across treatments (Figure 1D). Si-stressed environments oxylipin production may alleviate nutrient stress by increasing the dissolution of bSi and increasing the regeneration of inorganic phosphorus via enhanced APase activity. 

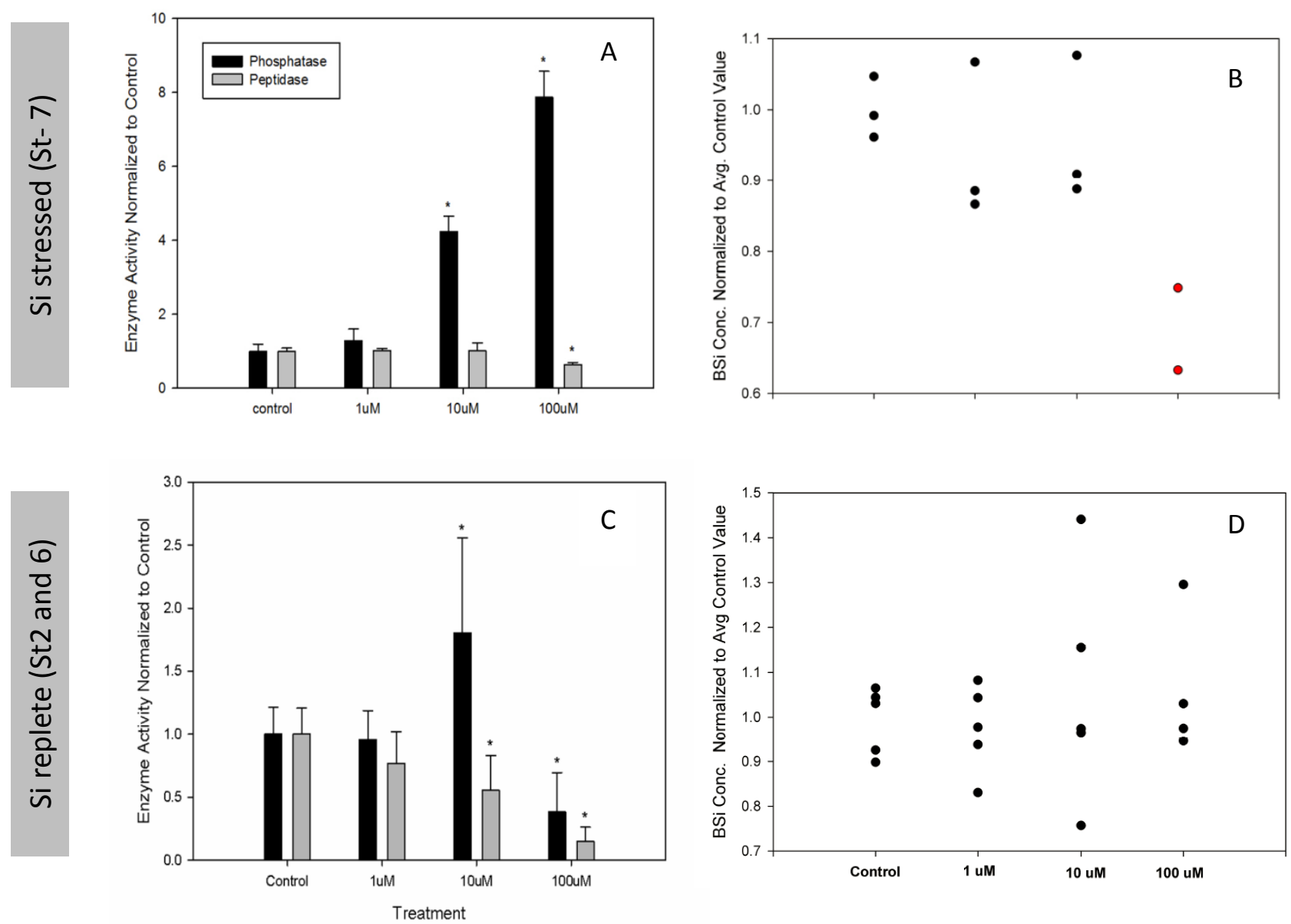

Figure 1. Varying response in enzymatic activity and biogenic dissolution depending on the state of Si-limitation in the upper water column A) Enzymatic activity of alkaline phosphatase and peptidase of trap material collected from Si-stressed waters and incubated with $0,1,10$, or 100 $\mu \mathrm{M}$ PUAs for 24 hours ( $\mathrm{N}=3$ for each treatment). Values were normalized to the mean of the control. Asterisk denotes significant difference from the mean. B) Biogenic silica dissolution presented as the concentration of $\mathrm{BSi}$ in each triplicate normalized to the average control concentration after incubation trap material for 24 hours with $0,1,10$, or $100 \mu \mathrm{M}$ PUAs $(\mathrm{N}=3$ for each treatment). Values below 1 denote dissolution. Red circles denote samples with significantly lower BSi (i.e. enhanced BSi dissolution). C) Enzymatic activity of alkaline phosphatase and peptidase of trap material collected from Si-replete waters and incubated with 0 , 1,10 , or $100 \mu \mathrm{M}$ PUAs for 24 hours ( $\mathrm{N}=6$ for each treatment). Values were normalized to the mean of the control. Asterisk denotes significant difference from the mean. D) Biogenic silica dissolution presented as the concentration of $\mathrm{BSi}$ in each triplicate normalized to the average control concentration after incubation trap material for 24 hours with $0,1,10$, or $100 \mu \mathrm{M}$ PUAs ( $\mathrm{N}=6$ for each treatment). Values below 1 denote dissolution. Red circles denote samples with significantly lower BSi (i.e. enhanced BSi dissolution). 


\section{References}

Bidle and Azam. 1999. Accelerated dissolution of diatom silica by marine bacterial assemblages.

Nature 397 (6719): 508-512 


\section{Appendix E}

Preliminary analysis of oxylipins produced intracellularly during infection of Chaetoceros tenuissimus with RNA virus. 
1.The particulate lipidome of Chaetoceros tenuissimus over the seven day course of infection by RNA virus analyzed with high resolution mass accuracy

Kim Thamatrakoln and Kay Bidle at Rutgers set up paralelle culture experiments of Chaetoceros tenuissimus with and without the RNA virus CtenRNAV in duplicate. Particulate lipid samples were collected at $\mathrm{t}=0,3,5$, and 7 days by filtering $10-20 \mathrm{~mL}$ of sample onto a $0.2 \mathrm{uM}$ durapore filter. Filters were frozen in liquid nitrogen and transported to WHOI in a dry shipper. Samples were extracted by Jeremy Tagliaferre using a modified Bligh and Dyer method (Popendorf et al 2011). All samples were analyzed on the LCQ, and single replicate from each day/treatment was analyzed on the Thermo Exactive Plus to give the high resolution data presented here.

Features were detected in the negative ion HPLC-HRAM mass spec data using MAVEN and putative annotations were assigned using the custom negative database. A preliminary look at the free fatty acids and oxylipins within the particulate lipidome revealed that over the course of infection free fatty acids associated with oxylipin biosynthesis decreased in the infected cells relative to the uninfected cells and intracellular concentrations of some oxylipins increased (Figure 1). This suggests that viral infection of diatoms increases oxylipin production relative to healthy cells. The dissolved lipidome was not sampled during this experiment but given the strong link between RNA viral abundance and oxylipins in the dissolved lipidome presented in Chapter 4, release of oxylipins into the dissolved phase should be the focus of future studies. 

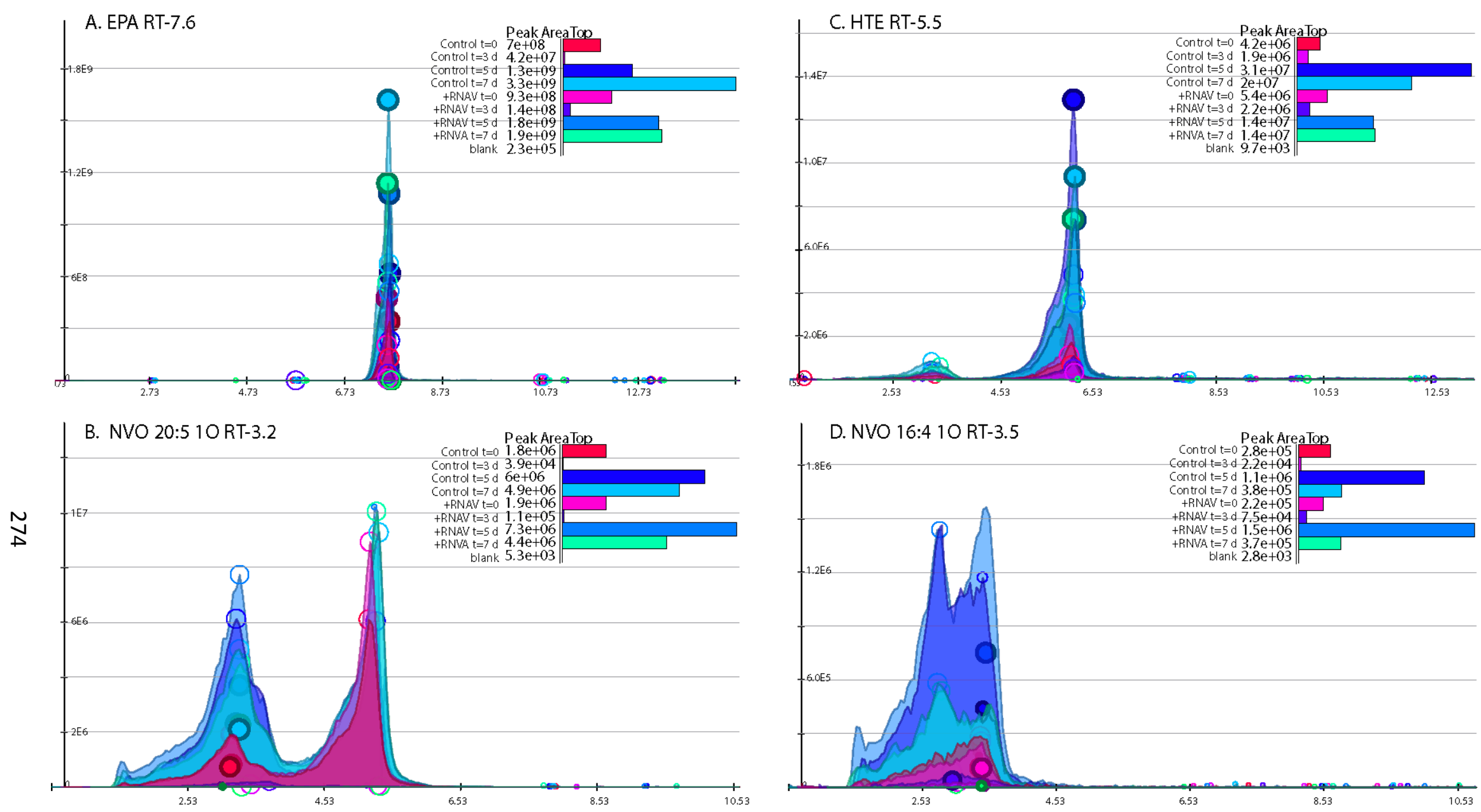

Figure 1. EIC of A) Eicosapentaenoic acid (EPA) B) hexadecatetraenoic acid (HTE), and the non-volatile oxylipins 20:5 1O and 16:4 10 within the particulate lipidome of $C$. ten without viruses (Control) at $\mathrm{t}=0,3$ days, 5 days, and 7 days as well as $C$. ten grown with RNA virus at $\mathrm{t}=0,3$ days, 5 days, and 7 days after infection. Peak area top values are normalized to the volume filtered and the cell concentration. Note that the peak top area of the free fatty acids EPA and HTE decreased intracellularly with infection and the nonvolatile oxylipins increased. 